\title{
ENHANCING RESILIENCE OF STEEL FRAME BUILDINGS USING SUPERELASTIC VISCOUS DAMPERS
}

\author{
A Dissertation \\ by \\ BAIKUNTHA SILWAL \\ Submitted to The School of Engineering and Applied Science of \\ The University of Virginia \\ In partial fulfillment of the requirements of the degree of \\ DOCTOR OF PHILOSOPHY \\ in \\ Civil Engineering
}

December 2017 


\title{
ENHANCING RESILIENCE OF STEEL FRAME BUILDINGS USING SUPERELASTIC VISCOUS DAMPERS
}

\author{
A Dissertation \\ by \\ BAIKUNTHA SILWAL \\ Submitted to The School of Engineering and Applied Science of \\ The University of Virginia \\ In partial fulfillment of the requirements of the degree of
}

DOCTOR OF PHILOSOPHY

in

Civil Engineering

Approved by:

Chair of Advisory Committee

Devin K. Harris

Committee Members

Osman E. Ozbulut (Advisor)

Jose Gomez

Kirk Martini

Steven Chase

July 2017 


\begin{abstract}
Enhancing Resilience of Steel Frame Buildings using Superelastic Viscous Dampers
\end{abstract}

Baikuntha Silwal, M.S., Southern Illinois University, Carbondale

Advisor: Dr. Osman E. Ozbulut

Conventional seismic design approaches rely on the ability of structures to dissipate the input earthquake energy through inelastic deformations in the designed regions of steel frames, implying substantial structural damage and potential residual drifts after a major earthquake event. Peak response quantities, such as peak story drifts and peak floor accelerations, are typically considered to evaluate the performance of different structural systems under seismic loads. However, several studies have shown that residual drifts, which occur due to the nonlinear behavior of yielding components of a structural system, may hold an important role in defining the performance of a structure after a seismic event and in the evaluation of potential damage. To enhance the seismic performance of structural systems, systems that can provide stable energy dissipation with full self-centering capabilities are desirable. These systems, known as selfcentering or re-centering, exhibit a flag-shaped hysteric response with the ability to return to small or zero deformation after each cycle. Such a self-centering system controls structural damage while minimizing residual drifts.

This dissertation proposes a hybrid passive control device and investigates its performance in improving the response of steel frame structures subjected to multi-level seismic hazards. The proposed superelastic viscous damper (SVD) relies on shape memory alloy (SMA) cables for recentering capability and employs a viscoelastic damper, which consists of two layers of a high damped blended butyl elastomer compound, to augment its energy dissipation capacity. First, the 
design and behavior of the proposed device are introduced. Then, the influences of various design parameters on the mechanical response of the device are investigated. Numerical models for the SVDs and steel moment frame buildings are developed in a finite element analysis program to determine the dynamic response of the structure to various levels of seismic hazards. The performance of steel structures retrofitted or newly designed with the installed SVDs is explored through nonlinear response history analyses. In addition, the seismic collapse resistance of steel frame buildings with SVDs is comparatively evaluated. The aftershock performance of steel frame buildings, with and without installed SVDs, is also investigated. Next, the effect of the ambient temperature on the performance of the proposed device is assessed. Finally, the seismic loss assessment of steel buildings with and without SVDs is conducted. It shows that steel buildings designed with SVDs have improved seismic performance and post-earthquake functionality. 


\section{DEDICATION}

To my loving father and mother,

for their endless support and words of inspiration 


\section{ACKNOWLEDGEMENTS}

I would like to express my sincere gratitude to my advisor, Dr. Osman Ozbulut, for providing me the opportunity to work with him at The University of Virginia, and for his guidance, motivation, and support throughout my study. I appreciate enormously his constant guidance and inspiration for my Doctoral program. I would like to thank my doctoral dissertation committee members, Dr. Devin Harris, Dr. Jose Gomez, Dr. Kirk Martini, and Dr. Steven Chase for their support and feedback throughout my study. Also, I would like to acknowledge the support I received from the Department of Civil and Environmental Engineering in pursuing graduate study at The University of Virginia.

I am continuously grateful to my family for the unconditional support and love I received; without their support I would not be here. I also extend grateful appreciation to my friends and colleagues who have helped me feel at home, as though I was in my native Nepal, during my time in Charlottesville. Finally, I am especially indebted to my wife Renuka, my son Projjwal, and my daughter Prisha for their support, love, and understanding through the past years. 


\section{NOMENCLATURE}

\begin{tabular}{|c|c|}
\hline \multicolumn{2}{|c|}{ ABBREVIATIONS } \\
\hline AISC & American Institute of Steel Construction \\
\hline ANSI & American National Standards Institute \\
\hline ASCE & American Society of Civil Engineers \\
\hline ASTM & American Society for Testing and Materials \\
\hline $\mathrm{BRB}$ & Buckling Restrained Brace \\
\hline CBFS & Concentrically Braced Frames \\
\hline CMR & Collapse Margin Ratio \\
\hline $\mathrm{CP}$ & Collapse Prevention \\
\hline $\mathrm{DBE}$ & Design-based Earthquake \\
\hline $\mathrm{DM}$ & Damage State \\
\hline EDP & Engineering Demand Parameter \\
\hline ERB & Elastomeric Rubber Bearings \\
\hline $\mathrm{FE}$ & Finite Element \\
\hline FEMA & Federal Emergency Management Agency \\
\hline FFT & Fast Fourier Transform \\
\hline FOE & Frequent Level of Earthquake \\
\hline HD & Highly Damped \\
\hline HDRB & High Damping Rubber Bearing \\
\hline HS & High Strength \\
\hline IDA & Incremental Dynamic Analysis \\
\hline IMs & Intensity Measures \\
\hline $\mathrm{IO}$ & Immediate Occupancy \\
\hline LRB & Lead Rubber Bearing \\
\hline $\mathrm{LS}$ & Life Safety \\
\hline MAFE & Mean Annual Frequency of Exceedance \\
\hline MANSIDE & $\begin{array}{l}\text { Memory Alloys for New Seismic Isolation and Energy Dissipation } \\
\text { Devices }\end{array}$ \\
\hline MAX & Maximum Response \\
\hline
\end{tabular}




$\begin{array}{ll}\text { MCE } & \text { Maximum Considered Earthquake } \\ \text { MIDR } & \text { Maximum Inter-story Drift Ratio } \\ \text { MRDR } & \text { Maximum Residual Drift Ratio } \\ \text { MTS } & \text { Material Testing System } \\ \text { NEHRP } & \text { National Earthquake Hazards Reduction Program } \\ \text { NP } & \text { Negative Polarity } \\ \text { NR } & \text { Natural Rubber } \\ \text { NRB } & \text { Natural Rubber Bearing } \\ \text { OpenSees } & \text { Open System for Earthquake Engineering Simulation } \\ \text { PACT } & \text { Performance Assessment Calculation Tool } \\ \text { PEER } & \text { Pacific Earthquake Engineering Research Center } \\ \text { PFA } & \text { Peak Floor Acceleration } \\ \text { PGA } & \text { Peak Ground Acceleration } \\ \text { PGV } & \text { Peak Ground Velocity } \\ \text { PIB } & \text { Polyisobutylene } \\ \text { PP } & \text { Positive Polarity } \\ \text { RBS } & \text { Reduced Beam Section } \\ \text { RHD } & \text { Reusable Hysteric Damper } \\ \text { RSMAD } & \text { Re-centering Shape Memory Alloy Damper } \\ \text { SC-BRB } & \text { Self-centering Buckling-restrained Brace } \\ \text { SDC } & \text { Seismic Design Category } \\ \text { SE } & \text { Superelasticity } \\ \text { SEI } & \text { Structural Engineering Institute } \\ \text { SFDB } & \text { Self-centering Friction Damping Brace } \\ \text { SMA } & \text { Shape Memory Alloy } \\ \text { SMA-LRB } & \text { SMA-based Lead Rubber Bearing } \\ \text { SMARB } & \text { SMA Supplement Elastomeric Rubber Bearing or } \\ \text { SME } & \text { SMA Supplemented Rubber Bearing } \\ \text { SMRF } & \text { SRB }\end{array}$


SSMAFD

SVD

VE

VED

\section{SYMBOLS}

$\delta$

$\gamma$

$\delta_{\text {SMA }}$

ESMA

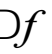

$\mathrm{A}_{\mathrm{f}}$

$\mathrm{Al}$

$\mathrm{A}_{\mathrm{s}}$

b

B

$\mathrm{Be}$

C

$\mathrm{C}_{\mathrm{i}}$

Co

$\mathrm{Cu}$

$\mathrm{d}_{\max }$

$\mathrm{d}_{\min }$

$\mathrm{D}_{\mathrm{s}}$

dsma

$\mathrm{E}_{\mathrm{D}}$

Es

$\mathrm{Fa}_{\mathrm{a}}$

$\mathrm{Fe}$

$\mathrm{f}_{\mathrm{i}}$
Superelastic Shape Memory Alloy Friction Damper

Superelastic Viscous Damper

Viscoelastic

Viscoelastic Damper

Design Displacement

Ratio of Post-transformation Hardening Stiffness to Initial Stiffness

Elongation of Shape Memory Alloy Cable

Strain on Shape Memory Alloy Cable

Frequency Interval

Austenite Finish Temperature

Aluminum

Austenite Start Temperature

Height of Shape Memory Alloy Element

Boron

Beryllium

Viscous Damping Coefficient

Fourier Amplitude

Cobalt

Copper

Maximum Cyclic Displacements

Minimum Cyclic Displacements

Significant Duration

Diameter of each Shape Memory Alloy Cable

Energy Dissipated per Cycle

Maximum Strain Energy for the Same Cycle

Forward Activation Force

Iron

Discrete Fourier Transform Frequencies 


\begin{tabular}{|c|c|}
\hline $\mathrm{F}_{\max }$ & Maximum Forces \\
\hline $\mathrm{F}_{\min }$ & Minimum Forces \\
\hline FNCATB & Fe-Ni-Co-Al-Ta-B \\
\hline $\mathrm{G}^{\prime \prime}$ & Dynamic Shear Modulus \\
\hline $\mathrm{G}^{\prime}$ & Elastic Shear Modulus \\
\hline K & Elastic Spring Coefficient \\
\hline $\mathrm{k}_{1}$ & Initial Stiffness \\
\hline $\mathrm{k}_{2}$ & Post-activation Stiffness \\
\hline $\mathrm{K}_{\mathrm{e}}$ & Dynamic Elastic Stiffness \\
\hline $\mathrm{K}_{\mathrm{s}}$ & Equivalent Stiffness \\
\hline$\eta$ & Loss Factor \\
\hline LSMA & Length of Shape Memory Alloy Cable \\
\hline $\mathrm{M}_{\mathrm{d}}$ & Austenite Stabilization Temperature \\
\hline $\mathrm{M}_{\mathrm{f}}$ & Martensite Finish Temperature \\
\hline $\mathrm{M}_{\mathrm{L}}$ & Local Magnitude \\
\hline $\mathrm{Mn}$ & Manganese \\
\hline $\mathrm{M}_{\mathrm{s}}$ & Martensite Start Temperature \\
\hline $\mathrm{M}_{\mathrm{w}}$ & Moment Magnitude \\
\hline $\mathrm{Nb}$ & Niobium \\
\hline $\mathrm{Ni}$ & Nickel \\
\hline NiTi & Nickel Titanium \\
\hline $\mathrm{N}_{\mathrm{SMA}}$ & Number of Shape Memory Alloy cables, \\
\hline $\mathrm{Pd}$ & Palladium \\
\hline rhc & Rubber Hydrocarbon \\
\hline $\mathrm{S}_{\mathrm{a}}\left(\mathrm{T}_{1}\right)$ & Spectral Acceleration at the Fundamental Period \\
\hline $\mathrm{S}_{\mathrm{CT}}$ & Median Collapse Capacity \\
\hline $\mathrm{Si}$ & Silicon \\
\hline $\mathrm{S}_{\mathrm{MT}}$ & $\begin{array}{l}\text { Median 5\%-damped Spectral Acceleration of the MCE ground } \\
\text { motions at the fundamental period of the building }\end{array}$ \\
\hline $\mathrm{T}$ & Temperature \\
\hline $\mathrm{Ta}$ & Tantalum \\
\hline
\end{tabular}


$\mathrm{Ti}$

$\mathrm{T}_{\mathrm{m}}$

$\mathrm{Zn}$

$\alpha$ $\beta$

$\delta_{c}$

$\rho$
Titanium

Mean Period

Zinc

Nonlinear Exponent Coefficient

Ratio of Forward to Reverse Activation Force

Collapse Limit

Correlation Coefficient 


\section{TABLE OF CONTENTS}

ABSTRACT ................................................................................................................................................ III

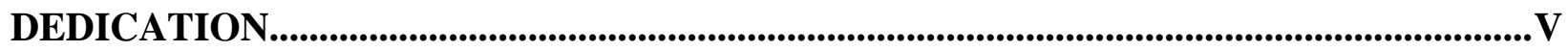

ACKNOWLEDGEMENTS _................................................................................................. VI

NOMENCLATURE.....................................................................................................VII

TABLE OF CONTENTS ...............................................................................................................XII

LIST OF TABLES ................................................................................................................................ XVI

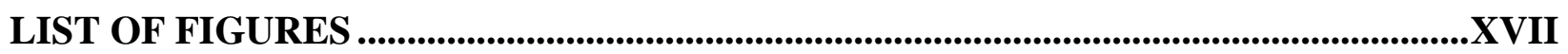

1 INTRODUCTION ...................................................................................................................

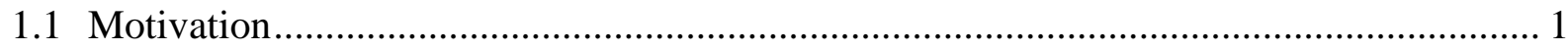

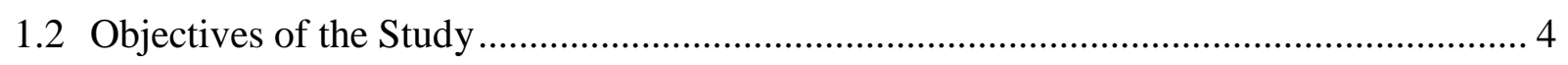

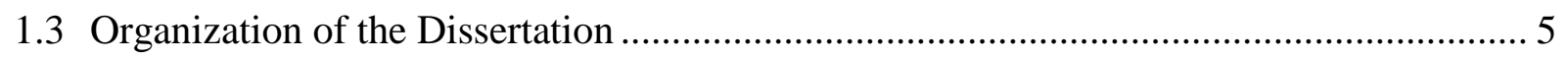

2 LITERATURE REVIEW ........................................................................................................

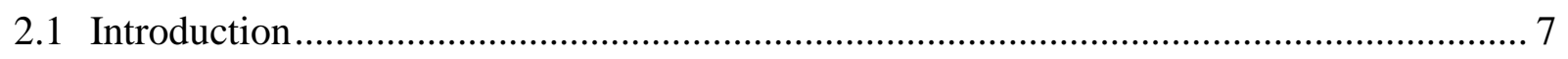

2.2 Shape Memory Alloy Characteristics ………………................................................... 7

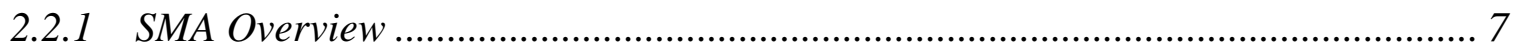

2.2.2 SMA Microstructure ……………………………………………………... 8

2.2.3 Shape Memory Effect ………………………....................................... 9

2.2.4 Superelastic Effect ................................................................................. 11

2.2.5 Shape Memory Alloy Compositions …………………………………………. 12

2.3 Mechanical Behavior of NiTi Shape Memory Alloys ..................................................... 15

2.3.1 General Characteristics of NiTi SMAs ……………..................................... 16

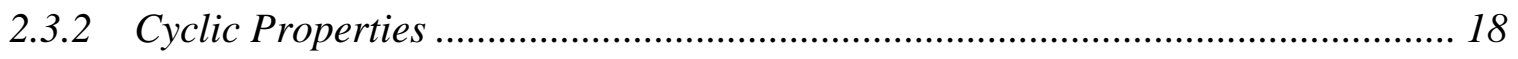

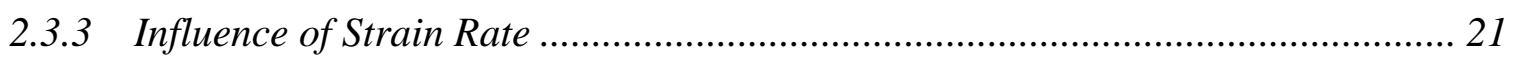

2.3.4 Influence of Temperature .................................................................................. 22

2.4 Applications of Shape Memory Alloys in Civil Structures .............................................. 23

2.4.1 SMA-based Structural Connections .................................................................. 24

2.4.2 SMA-based Bracing Systems........................................................................ 29 


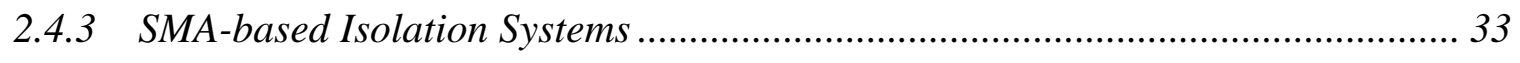

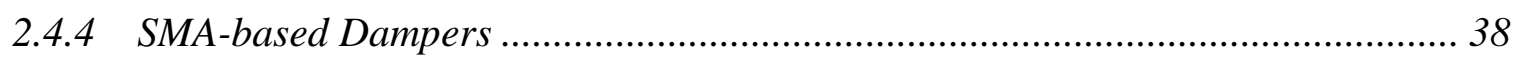

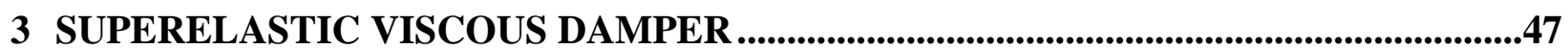

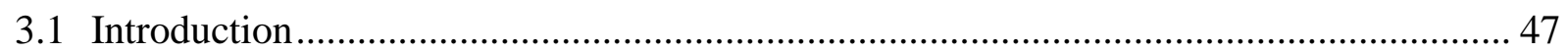

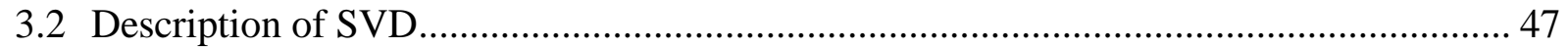

3.2.1 Experimental Characterization of SMA Cables.................................................... 47

3.2.2 Experimental Characterization of High Damped Butyl Elastomer ...................... 50

3.2.3 Superelastic Viscous Damper ............................................................................ 55

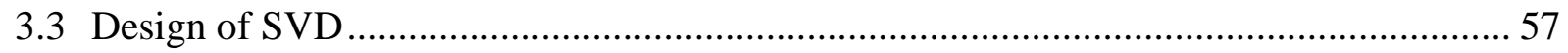

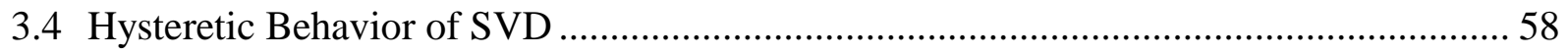

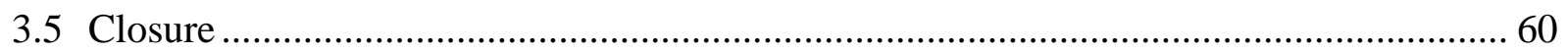

4 SEISMIC RETROFITTING OF STEEL FRAME BUILDINGS WITH SVDS................61

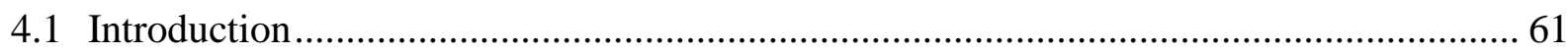

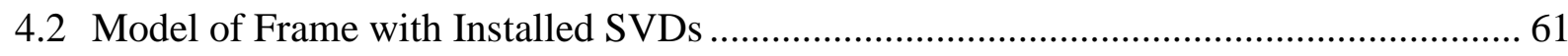

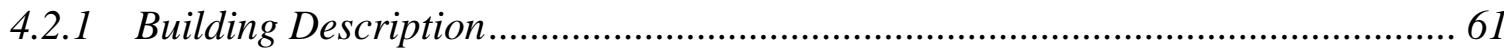

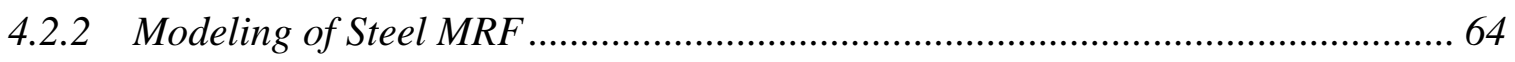

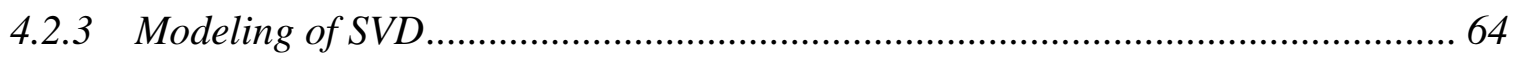

4.2.4 Design of Steel MRF with SVDs ...................................................................... 67

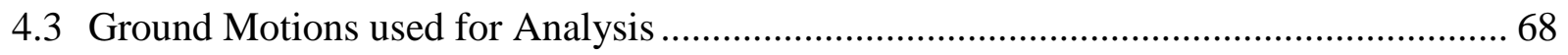

4.4 Performance Assessment ........................................................................................... 69

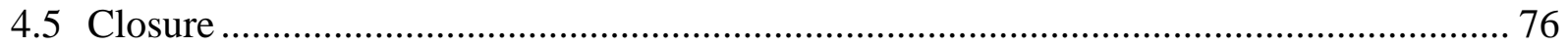

\section{SEISMIC COLLAPSE ASSESSMENT OF STEEL FRAME BUILDINGS WITH}

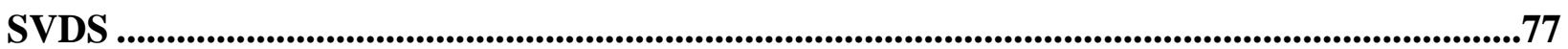

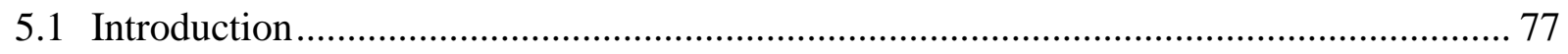

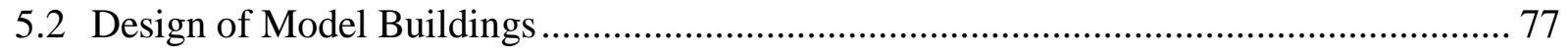

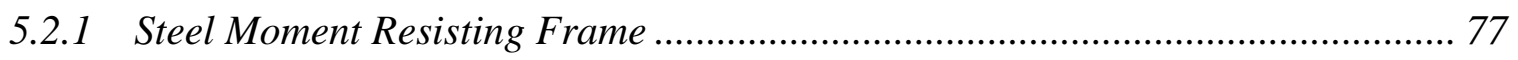

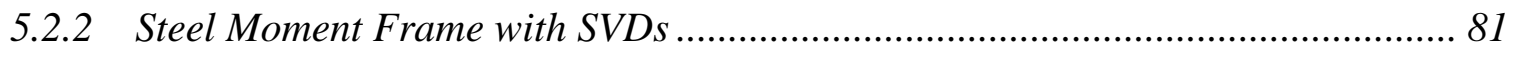

5.2.3 Steel Moment Frame with BRBs ..................................................................... 83

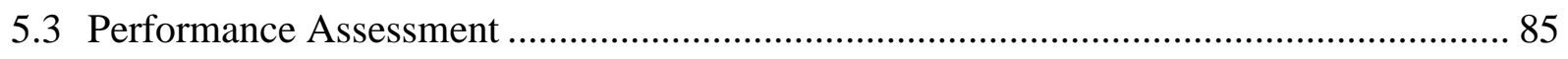

5.3.1 Ground Motion Records ……………………............................................... 85 


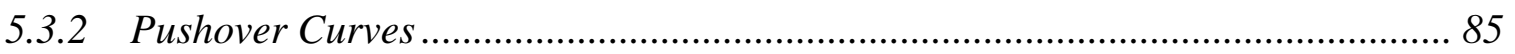

5.3.3 Incremental Dynamic Analysis ....................................................................... 86

5.3.4 Influence of SVD parameters ............................................................................ 93

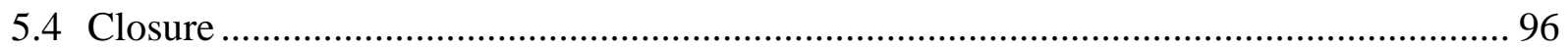

6 SEISMIC FRAGILITY ESTIMATES OF STEEL FRAME BUILDINGS WITH

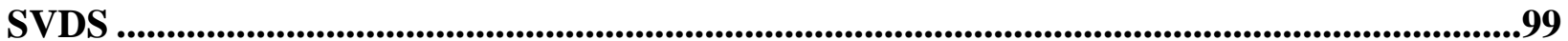

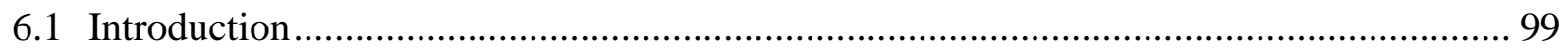

6.2 Building Description and Modeling............................................................................ 99

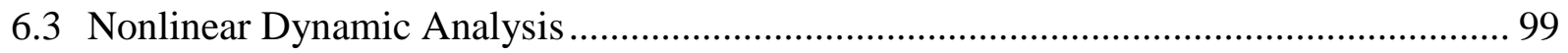

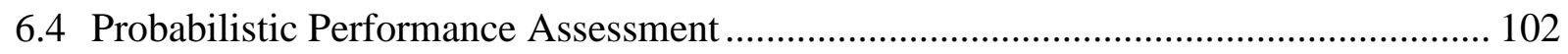

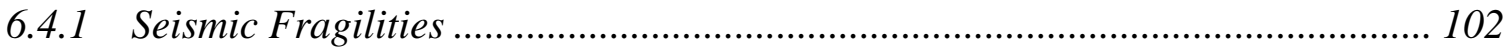

6.4.2 Probabilistic Model Developments of Engineering Demand Parameters.......... 103

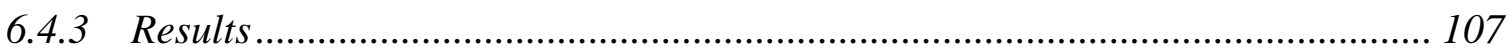

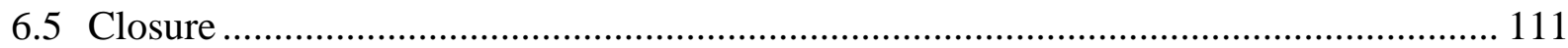

7 MAINSHOCK-AFTERSHOCK PERFORMANE EVALUATION OF STEEL FRAME

BUILDINGS WITH SVDS ..........................................................................................................113

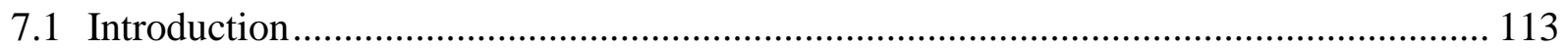

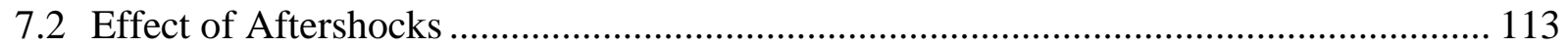

7.3 Aftershock Performance Assessment Framework ....................................................... 116

7.4 Numerical Modeling and Design of Steel Moment Frames ........................................... 117

7.5 Selection of Ground Motion Sequences …………….................................................. 117

7.6 Performance Assessment for Mainshock-Aftershock Analysis....................................... 121

7.6.1 Mainshock Seismic Assessment..................................................................... 121

7.6.2 Aftershock Seismic Assessment ....................................................................... 122

7.6.3 Demolition Capacity Assessment ............................................................... 126

7.6.4 Effect of Aftershock Polarity.......................................................................... 127

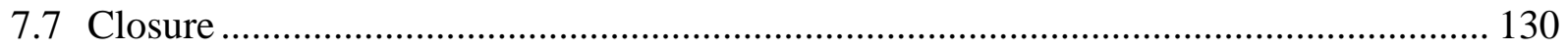

8 EFFECT OF TEMPERATURE ON SEISMIC PERFORMANCE OF SVDS .................133

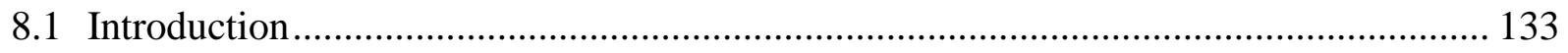

8.2 Temperature Effects on Butyl Rubber …………….................................................. 133 
8.3 Temperature Effects on Shape Memory Alloys..................................................... 135

8.4 Building Description and Numerical Modeling ...................................................... 137

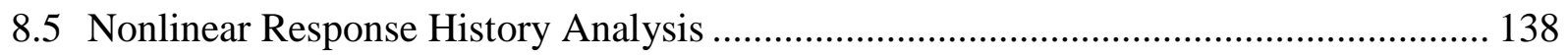

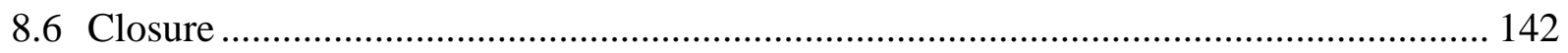

9 SEISMIC LOSS ASSESSMENT OF STEEL FRAME BUILDINGS WITH SVDS ......143

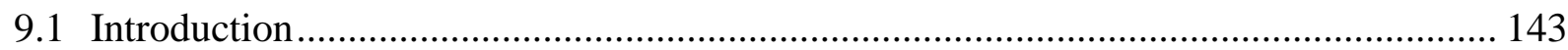

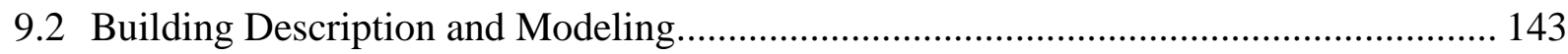

9.3 Seismic Loss Assessment Methodology …........................................................... 143

9.4 Seismic Hazard Curve ............................................................................................. 146

9.5 Nonlinear Response History Analysis ............................................................. 148

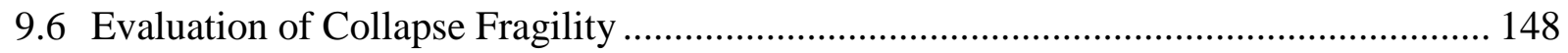

9.7 Performance Assessment Results ........................................................................... 150

9.7.1 PACT Results for Repair Cost and Repair Time ......................................... 150

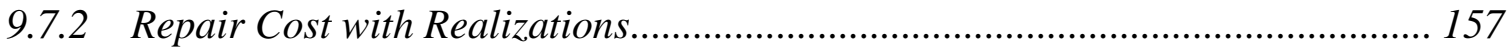

9.7.3 Disaggregation of Building Loss ................................................................. 160

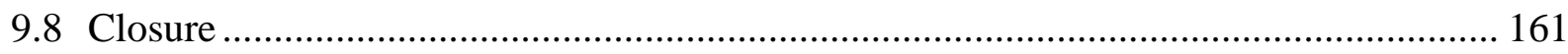

10SUMMARY, CONCLUSIONS, AND RECOMMENDATIONS..................................163

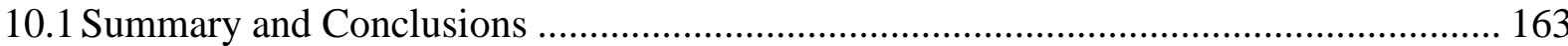

10.2 Recommendations for Future Studies ............................................................... 167

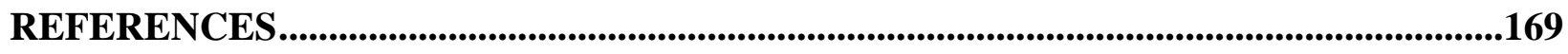

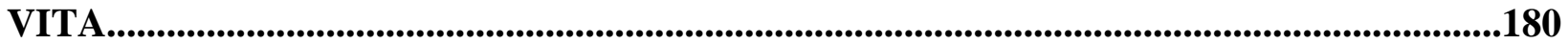




\section{LIST OF TABLES}

Table 2-1 Comparative advantages of three shape memory alloy compositions....................... 15

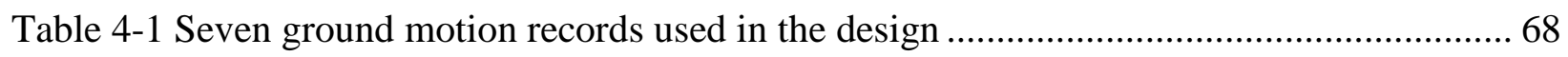

Table 5-1 Members of steel moment resisting frames ................................................... 82

Table 5-2 Mean story drifts under 7 design ground motions at DBE and MCE level................. 84

Table 5-3 Collapse safety parameters for different frames................................................ 88

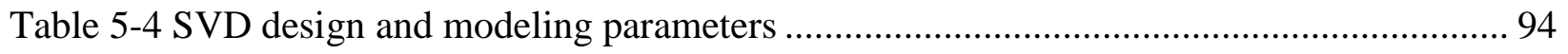

Table 5-5 Collapse safety parameters for frames with different SVD designs....................... 94

Table 6-1 Statistics of model parameters in the peak inter-story drift (k= ID) and residual inter-

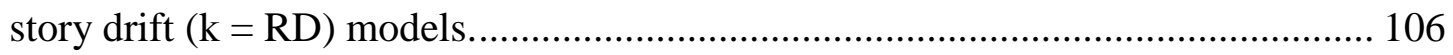

Table 6-2 Statistics of model parameters in the peak floor acceleration (k= PFA) models. ..... 106

Table 6-3 Medium of inter-story drift, residual inter-story drift, and peak floor acceleration capacities for various performance levels...................................................... 107

Table 7-1 Seismic sequences and their characteristics ................................................... 119

Table 7-2 Median collapse capacities for SMRF and SVD under seismic sequences............... 124

Table 8-1 Model parameters for butyl rubber and SMAs for different temperatures................ 138

Table 8-2 Ground motion records used in analyses........................................................ 139

Table 9-1 The hazard curve data at the selected intensity level ........................................ 147 


\section{LIST OF FIGURES}

Figure 1-1 Damages to steel structures during earthquake events (a) 3-story steel moment resisting frame with a three-story complete collapse mechanism (2011 Tohoku Japan) (EERI, 2011); (b) 21-story steel frame office building (1985 Mexico) (wikiwand, 1985) ... 2

Figure 2-1 Different phases of shape memory alloy microstructure .................................... 9

Figure 2-2 SMA microstructure representation of shape memory effect and superelasticity ...... 10

Figure 2-3 Temperature and deformation relationship of SMA microstructure for shape memory

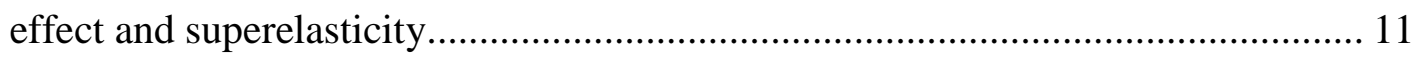

Figure 2-4 Stress-strain relationship for shape memory effect in SMAs................................ 12

Figure 2-5 Stress-strain relationship for superelastic SMAs ............................................... 13

Figure 2-6 (a) Comparison of elastic modulus and recovery strain and (b) comparison of hysteretic response of different SMAs (Billah AHMM, 2015) ........................................ 17

Figure 2-7 Results of cyclic tensile tests on NiTi wires (Malecot et al., 2006)......................... 19

Figure 2-8 Hysteresis cycles of stress-strain curves: (a) response curves of the single Nitinol wire; (b) response of the seven-wire Nitinol strand (Reedlunn et al., 2013) .................. 21

Figure 2-9 Stress-strain curves of NiTi wires at different temperatures (Churchill et al., 2009) . 23

Figure 2-10 Innovative Steel Beam-Column Connection Using Shape Memory Alloys Tendons

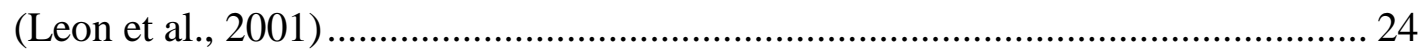

Figure 2-11 The SMA connection diagram (Ma et al., 2007) ............................................. 26

Figure 2-12 Steel beam-column connection details (Speicher et al., 2011) ............................. 28

Figure 2-13 Geometric configurations and layouts (Yam et al., 2015) ................................... 29

Figure 2-14 Bracing system with SMA connection in two cases (Asgarian and Moradi, 2011) . 31

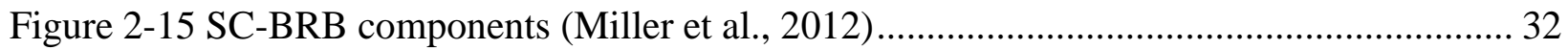

Figure 2-16 Mechanism of the stopper: deformation of the shape memory alloy (SMA) bar (Araki et al., 2016) 33 
Figure 2-17 A bridge structure modeled with sliding bearings and SMA device (Ozbulut and Hurlebaus, 2010) 34

Figure 2-18 Description of the isolation bearing (a) HDRB; the rubber layers with high-damping properties are vulcanized by steel shims, (b) SRB in un-deformed condition, and (c) SRB in deformed condition (Bhuiyan and Alam, 2013).................................... 35

Figure 2-19 Configurations of the SMARB system: (a) shape-memory alloy (SMA) cable/bar supplementing RB and (b) the rubber bearing with SMA wrap (Gur et al., 2014) . 36

Figure 2-20 Configuration of the isolation system supplemented with SMA (Shinozuka et al., 2015) 37

Figure 2-21 (a) SMA supplement elastomeric rubber bearing (SMARB) and (b) Installation of SMARB at the base isolated building frame (Ghodke and Jangid, 2017) .............. 38

Figure 2-22 Schematics of the (a) SMA-based reusable hysteretic damper, (b) an RHD in a steel framed building (Zhang and Zhu, 2007) ....................................................... 39

Figure 2-23 Schematic of mechanical configuration of SFDB (Zhu and Zhang, 2008)............ 40

Figure 2-24 Schematic diagram of the SMA damper (Ma and Cho, 2008).............................. 41

Figure 2-25 Proposed RSMAD device (Qian et al., 2013) ................................................... 42

Figure 2-26 (a) SMA wires inside a hybrid device with clevis pins for chevron bracing; (b) SMA wires inside a hybrid device with clevis pins for diagonal bracing; (c) arrangement of hybrid devices in chevron bracing; (d) arrangement of hybrid devices in diagonal bracing (Yang et al., 2010) ............................................................................. 43

Figure 2-27 Scheme diagram of SSMAFD (Qian et al., 2016) ............................................ 44

Figure 2-28 Schematic configuration of the superelastic damper (Bhaowmick and Mishra, 2016)

Figure 3-1. Shape memory cable: cross-sectional and longitudinal views. 48

Figure 3-2. Stress-strain curves of SMA cable under different (a) strain amplitudes and (b) loading frequencies. 
Figure 3-3. (a) Static shear stress versus shear strain, (b) Hysteresis loops at a frequency of $1 \mathrm{~Hz}$

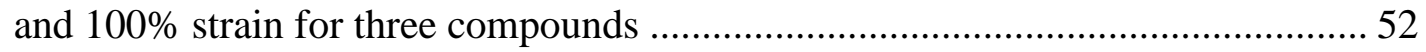

Figure 3-4. Shear modulus, elastic stiffness and loss factor for three compounds .................... 53

Figure 3-5. Hysteresis loops for HD butyl (a) at various strain amplitudes at $1 \mathrm{~Hz}$, and at various frequencies at (b) 50\% strain, (c) 100\% strain, and (d) 200\% strain. .................... 54

Figure 3-6. Variation of shear modulus, elastic stiffness and loss factor for HD butyl with loading frequency 55

Figure 3-7. (a) 3D rendering of SVD, (b) a schematic diagram of SVD at its undeformed and

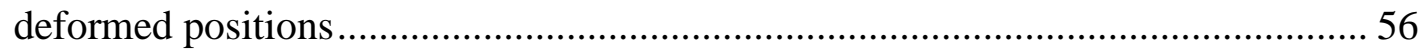

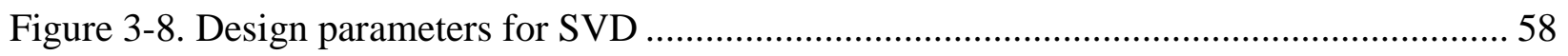

Figure 3-9. Force-deformation curves of the SVD and its sub-components (SMA and VED) at two different displacement amplitudes ............................................................... 59

Figure 4-1. Six-story steel special moment resisting frame: plan and elevation ...................... 63

Figure 4-2 Modified Ibarra-Krawinkler Hysteretic Model: (a) monotonic and (b) cyclic ........... 65

Figure 4-3. Parameters used to model SMA cables and butyl rubber in OpenSees ................... 66

Figure 4-4. Experimental stress-strain curve and model prediction for (a) SMA cable, (b) VE

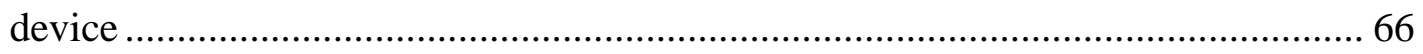

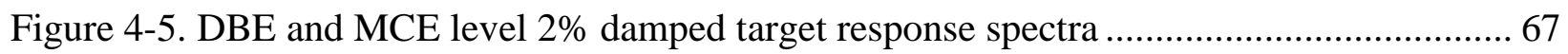

Figure 4-6. Acceleration spectra of individual ground motions for $2 \%$ damping and median spectrum

Figure 4-7. Peak inter-story drift, peak residual story drift, and peak story acceleration for individual DBE level ground motions 71

Figure 4-8. Peak inter-story drift, peak residual story drift, and peak story acceleration for individual MCE level ground motions

Figure 4-9. Statistics of peak inter-story drift ratio and peak story absolute acceleration for uncontrolled and controlled buildings subjected to 44 ground motions at (a) DBE level and (b)MCE level 
Figure 4-10. Time histories of selected response for uncontrolled and controlled structures subjected to MCE level Northridge earthquake and corresponding force-deformation curves for SVD and its subcomponents 75

Figure 4-11. Time histories of selected response for uncontrolled and controlled structures subjected to MCE level Kobe earthquake and corresponding force-deformation curves for SVD and its subcomponents 75

Figure 5-1. Plan and elevation of nine-story steel special moment resisting frame 79

Figure 5-2 Schematic numerical model of the partial steel frame in OpenSees ....................... 81

Figure 5-3. Superelastic viscous dampers installed into steel frame 82

Figure 5-4. (a) Components of buckling restrained brace and (b) BRB installed to steel frame.. 84 Figure 5-5. Acceleration spectra of each ground motion for 5\% damping and median spectrum 85 Figure 5-6. Pushover curves for three different steel frame systems 86

Figure 5-7. Maximum inter-story drift ratio IDA curves for: (a) SMRF, (b) SVD frame, and (c) BRB frame 87

Figure 5-8. Maximum floor acceleration IDA curves for: (a) SMRF, (b) SVD frame, and (c) BRB frame 89

Figure 5-9. Maximum residual drift ratio IDA curves for: (a) SMRF, (b) SVD frame, and (c) BRB frame 90

Figure 5-10. Profiles of (a) peak inter-story drift ratio, (b) peak floor acceleration, and (c) residual drift ratio at MCE level for each frame under individual ground motion records ... 91

Figure 5-11. Envelopes for median of peak response quantities for SMRF, SVD, and BRB frames at MCE level 92

Figure 5-12. Median peak response quantities under 44 ground motion records scaled to different intensity levels for SMRF, SVD, and BRB frames 93

Figure 5-13. Median peak response quantities under 44 ground motion records scaled to different intensity levels for different designs of SVD frame 95 
Figure 6-1 (a) Acceleration spectra of individual records and medium spectrum for 5\% damping,

(b) median spectrum scaled to different hazard levels. 100

Figure 6-2 Time histories of inter-story drift for SMRF and SVD frames under Northridge and Kobe earthquakes. 101

Figure 6-3 Time histories of top floor acceleration for SMRF and SVD frames under Northridge and Kobe earthquakes. 102

Figure 6-4.Fragility curves of structural components 109

Figure 6-5 Fragility curves of nonstructural drift sensitive components 110

Figure 6-6 Fragility curves of nonstructural acceleration sensitive components 110

Figure 7-1 Major aftershocks (a) within 30 days of April 2015 Nepal earthquake and (b) within 2 days of February 2010 Chile earthquake 114

Figure 7-2 Response spectrum of selected seismic sequences 120

Figure 7-3 Acceleration time history for a mainshock-aftershock sequence recorded at Convict Creek Station from 1980 Mammoth Lake Earthquake. 120

Figure 7-4 Incremental dynamic analysis curves of frames subjected to mainshocks: (a) SMRF, and (b) SVD frames 122

Figure 7-5 Maximum inter-story drift versus spectral acceleration IDA curve under mainshockaftershock sequence indicating three damage states for the SMRF and SVD frames 123

Figure 7-6 Median IDA curves at three damage states for SMRF and SVD frame under seismic sequences 125

Figure 7-7 Comparative fragility curves for SMRF and SVD frames at three damage states: (a) DS1, (b) DS2, and (c) DS3 126

Figure 7-8 Median aftershock IDA residual drift curves for SMRF and SVD frames at different mainshock damage levels 127

Figure 7-9 Time histories of fist floor drift for (a) SMRF and (b) SVD frames subjected to sequence S2 128 
Figure 7-10 Median aftershock IDA curves for maximum inter-story drift for SMRF and SVD frames at different mainshock damage levels considering aftershock polarity .... 129

Figure 7-11 Median aftershock IDA curves for maximum residual drift for SMRF and SVD frames at different mainshock damage levels considering aftershock polarity ................ 130

Figure 8-1 Shear stress - shear strain curves of HD butyl rubber at different temperatures ...... 134

Figure 8-2 Variation of equivalent stiffness and loss factor for HD butyl with temperature ..... 135

Figure 8-3 Stress - strain curves of SMAs at different temperatures ................................... 136

Figure 8-4 Variation of equivalent stiffness and loss factor for SMA with temperature .......... 137

Figure 8-5 Envelopes for median of peak response quantities for SVD systems at different temperatures at DBE level hazard.............................................................. 140

Figure 8-6 Envelopes for median of peak response quantities for SVD systems at different temperatures at MCE level hazard.....

Figure 8-7 Force-displacement curves of SVDs at different temperatures under Imperial Valley earthquake

Figure 9-1 FEMA P-58 Procedure for seismic loss assessment 144

Figure 9-2 Performance calculation process in each realization (FEMA P-58-1)..... 145

Figure 9-3 Seismic hazard curve for a nine-story steel frame located in Seattle. 146

Figure 9-4 Hazard curve at selected intensity measure used in PACT. 147

Figure 9-5 Collapse fragility curves for the SMRF and SVD frames. 150

Figure 9-6 Comparative repair costs and repair times for SMRF and SVD frames at different hazard levels. 151

Figure 9-7 Repair cost at DBE level: (a) SMRF and (b) SVD frames 153

Figure 9-8 Repair cost at MCE level: (a) SMRF and (b) SVD frames 154

Figure 9-9 Story-wise distribution of repair times at DBE level: (a) SMRF and (b) SVD frames 155 
Figure 9-10 Story-wise distribution of repair times at MCE level: (a) SMRF and (b) SVD frames 156

Figure 9-11 Repair costs from 200 realizations at the DBE level for (a) SMRF and (b) SVD frames 158

Figure 9-12 Repair costs from 200 realizations at the MCE level for (a) SMRF and (b) SVD frames 159

Figure 9-13 Disaggregation of the building loss curve into contributions from different intensity measures for SMRF 160

Figure 9-14 Disaggregation of the building loss curve into contributions from different intensity measures for the SVD frame. 161 



\section{INTRODUCTION}

\subsection{Motivation}

Conventional seismic design approaches rely on the ability of structures to dissipate input earthquake energy through inelastic deformations in the designed regions of the steel frames, implying substantial structural damage and potential residual drifts after a major earthquake event. The traditional design approach does not explicitly limit the amount of structural damage that is imperative to minimize the risk associated with significant economic losses, especially at high seismic regions. Recent earthquake events have demonstrated the limitations of conventional design methods, revealing the damaging effects on civil infrastructures caused by strong ground motion events as well as significant economic losses. Numerous frame structures were damaged or collapsed during major earthquake events such as the 1994 Northridge, 1995 Kobe, 1999 Duzce, 1999 Chi Chi, 2008 Winchuan, 2010 Chile, and 2011 Christchurch earthquakes. Figure 1-1 illustrates the damage to steel frame structures during 2011 Tohoku, Japan and 1985 Mexico earthquakes. The economic losses due to major earthquake events were also significant: \$100 billion, $\$ 30$ billion, and \$20 billion due to 1995 Kobe earthquake, 2010 Chile earthquake, and 1994 Northridge earthquake, respectively (USGS, 2017).

To overcome the limitations of the existing design approach, numerous innovative systems, devices and materials have been developed and tested for enhanced seismic protection of structures. As very reliable techniques for mitigating the seismic response on structural systems, passive control devices have been the most attractive method, with a rapid increase in the implementation of these devices over the past decades, although active and semi-active systems have also been explored for seismic response control. These passive devices, which require no additional energy to operate, generate a control force or provide improved energy dissipation in structural systems. A variety of passive systems such as friction dampers, metallic yielding dampers, fluid viscous dampers and viscoelastic dampers have been developed. Although each device has its merits for improving the performance of structures, each system also reveals its own limitations, such as the long-term behavior and durability problems of friction devices, the limited number of working cycles and need to replace yielding devices, and difficulty in reducing the peak structural response in the early stages of loading in the case of viscous devices. 


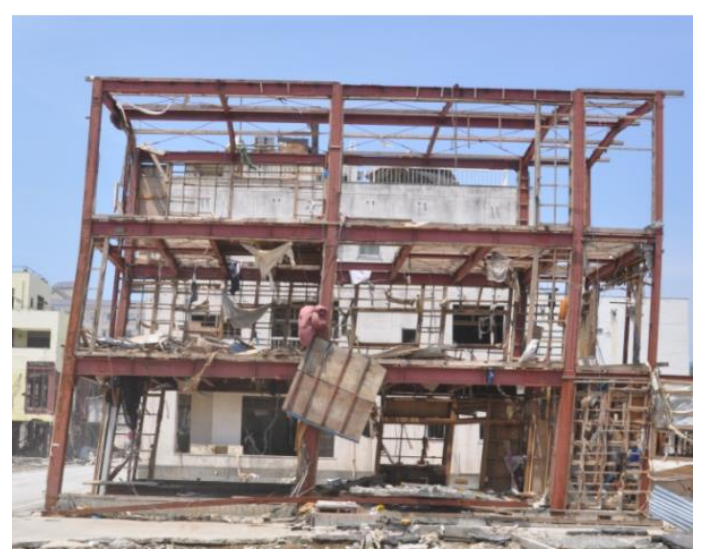

(a)

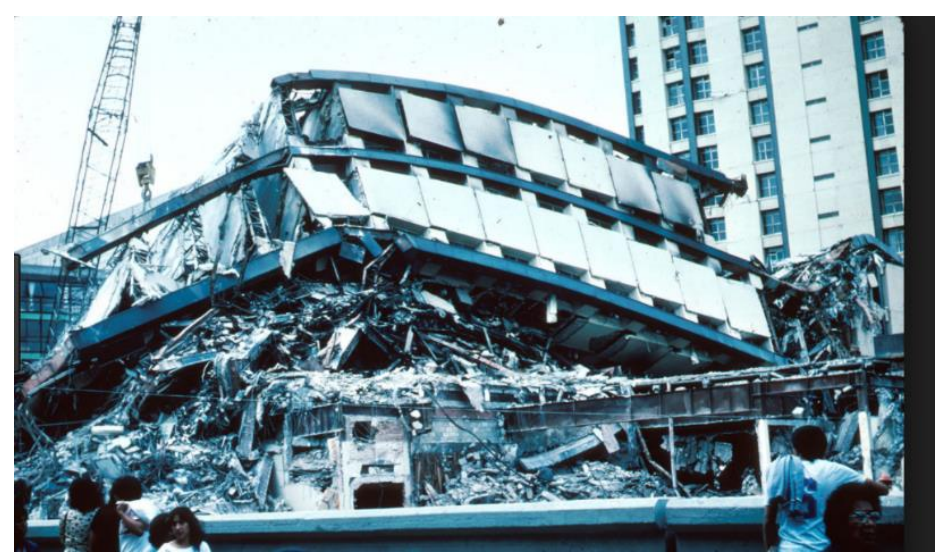

(b)

Figure 1-1 Damages to steel structures during earthquake events (a) 3-story steel moment resisting frame with a three-story complete collapse mechanism (2011 Tohoku Japan) (EERI, 2011); (b) 21-story steel frame office building (1985 Mexico) (wikiwand, 1985)

Although modern seismic design codes allow engineers to design building structures to undergo ductile inelastic deformations during a design-based earthquake, observation of significant residual drift in previous earthquake events has raised concerns about minimizing damage by reducing residual drift (Bojorque and Ruiz-Garcia, 2013). Since residual drift is recognized as a complementary parameter in evaluating structural performance, decreasing residual drift is critically imperative to reduce post-earthquake repair costs and to maximize postevent functionality. McCormick et al. (2008) studied the effects of residual drift on occupants, and concluded that residual drift greater than $0.5 \%$ in buildings may suggest a complete loss of the structure from an economic perspective. In another study, Erochko et al. (2010) examined the residual drift response of special moment resisting frames (SMRFs) and buckling-restrained braced frames (BRBs). The results indicated that both types of building systems experience significant residual drift: $0.8-1.5 \%$ for the SMRFs and 0.8-2.0\% for the BRBFs under design-based seismic hazards. In another study, Ramirez and Miranda (2012) found that considering residual drift in building earthquake loss estimation significantly increases expected economic losses. By reducing the residual drift of a structure subjected to a seismic event, structural engineers can 
maximize post-event functionality, reduce the cost to repair the structures, and increase public safety.

To enhance the seismic performance of structural systems, those that can provide stable energy dissipation with full self-centering capabilities are desirable. These systems, known as selfcentering or re-centering, exhibit the flag-shaped hysteric response with the ability to return to small or zero deformation after each cycle. The self-centering system can control structural damage while minimizing residual drift. There exist several different mechanisms for creating a restoring force to return a building to plumb after an earthquake. One approach is to allow structures to undergo controlled rocking at discrete locations, such as column-base joints or beamcolumn joints. These systems usually involve the use of unbonded post-tensioned tendons to provide bilinear elastic self-centering behavior and a dissipating system to produce sufficient energy dissipation. Rocking precast concrete walls (Holden et al., 2003; Perez et al., 2007) and post-tensioned precast concrete special moment frames (Priestley, 1996) are examples of such systems. Another approach is to employ braces or seismic control devices with self-centering capabilities. A number of such bracing systems or devices have been developed and studied (Christopoulos et al., 2008; Ghassemieh and Kargarmoakhar, 2013; Karavasilis et al., 2011; Dyanati et al., 2015; Bhowmick et al., 2016). An extensive review of self-centering systems can be found in Chancellor et al., 2014.

Shape memory alloys (SMAs) have attracted a great deal of attention as smart materials to be used in seismic protection systems for energy dissipating and re-centering purposes (Eatherton and Hajjar, 2011; Ozbulut et al., 2011; Qian et al., 2009). SMAs behave similarly to linear-elastic materials for small-magnitude events, but for moderate and more severe strain levels, SMAs display superelastic behavior from which they can fully recover their original elastic shape upon unloading. SMAs also exhibit self-centering behavior when permanent deformations in surrounding assemblies afflict the SMA installation; thus, the overall integrity of neighboring structural systems can be maintained. Due to the strain hardening of SMA materials, the stiffness of the overall system increases during extreme deformations, which further alleviates deflections in surrounding structural systems. Because of their re-centering capability, SMAs can serve as valuable components in a seismic control device. 
Previous studies indicate that the quantity of equivalent viscous damping provided by superelastic SMA wires or bars is not sufficient to justify the use of SMAs as the sole damping device implemented in a tall structure subjected to severe dynamic loadings (Desroches et al., 2004; McCormic et al., 2006; Ozbulut and Hurlebaus, 2010). Several researchers have explored the development of SMA-based control devices with supplemental energy dissipation capabilities. Yang et al. (2010) proposed a hybrid device that combines re-centering SMA wires with energyabsorbing steel struts. The device also utilizes two high-strength steel tubes to guide the movement of SMA wires and struts. Speicher et al. (2009) designed a tension/compression device for the seismic retrofit of building structures. This device was developed by the use of NiTi helical springs or NiTi Belleville washers in the compression. The results of cyclic loading tests suggested that the helical springs have good re-centering and damping characteristics, while Belleville washers can be used for energy dissipation purposes in an SMA device. Similarly, Zhang and Zhu (2008) investigated an SMA-based device, labeled a reusable hysteretic damper (RHD). The device comprised two blocks that slide past each other, and superelastic NiTi wires attached to the sliding blocks. Miller et al. (2012) developed and tested a self-centering buckling restrained brace (BRB) that combined a typical BRB component with pre-strained SMA rods. Luo et al. (2012) proposed an SMA damper that consisted of two components. Superelastic SMA wires were used as a recentering component, and martensite SMA sheet or low-yield steel sheet was used for energy dissipation.

Although SMA-based damping devices have shown promising performance in seismic response mitigation, further investigations and detailed performance validations of these systems are needed to facilitate real implementation. This study proposes the combination of SMA cables and a viscoelastic element in parallel for improved re-centering and energy dissipating capabilities. Compared to conventional structural control devices, the proposed SMA-based re-centering device is an attractive alternative in both performance and design efficiency.

\subsection{Objectives of the Study}

The goal of this research work is to design and characterize a hybrid passive control device, and evaluate its performance under multi-level seismic hazards. The device, named the superelastic viscous damper (SVD), combines the energy-dissipating capacity of a conventional 
viscoelastic damper with the excellent re-centering properties of SMA cables in a single device that demonstrates an improved, hybrid response. The advantageous attributes of the proposed device include large and scalable force capacity; excellent re-centering ability; high damping capacity; passive nature; and need for no special maintenance or replacement throughout the lifecycle. This study entails the following major tasks:

1) Evaluate and model the mechanical response of shape memory alloy cables and high damped butyl rubber compound;

2) Design a hybrid passive control device (SVD);

3) Evaluate the performance of SVDs in retrofitting a steel moment resisting frame;

4) Conduct a parametric study to evaluate the influence of design parameters on the mechanical behavior of an SVD;

5) Perform the comparative collapse performance evaluation for steel frames designed with SVDs;

6) Develop demand models and fragility curves for steel frames designed with SVDs;

7) Examine the mainshock - aftershock performance of steel frames designed with SVDs;

8) Evaluate the performance of SVDs, considering environmental temperature variations;

9) Conduct a seismic loss analysis for steel frames designed with SVDs.

The results of this study provide a better understanding of the behavior of structures with

re-centering devices, thus enabling engineers to design structures more effectively and improve the damage resistance and service life of steel frame systems.

\subsection{Organization of the Dissertation}

This dissertation consists of ten sections that are organized as follows:

Section 1 provides a brief discussion of research problems with the motivation and the scope of the research.

Section 2 presents an overview of shape memory alloys and a review of the application of SMAs into civil structures relevant to this study.

Section 3 describes the mechanical behavior of SMA cables and high damped butyl rubber and the conceptual design and hysteretic mechanics of SVDs. 
Section 4 explores the effectiveness of the proposed device in retrofitting a six-story steel frame building under multi-level earthquake events.

Section 5 compares the collapse performances of three lateral load-rresisting structural systems: special moment resisting frame, steel frame equipped with SVDs, and the steel frame system with buckling restrained braces.

Section 6 investigates the probabilistic seismic performance evaluation of the SVD system, and develops fragility curves of steel moment resisting frame with and without SVDs.

Section 7 includes the mainshock - aftershock performance evaluation of frame systems with and without SVDs.

Section 8 evaluates the effects of environmental temperature variations on the performance of SVDs.

Section 9 discusses a performance-based seismic loss analysis on steel moment resisting frames with and without SVDs.

Section 10 summarizes the dissertation work by presenting conclusions, and offers recommendations for the use of SMAs as a passive control system based on the findings of this study. 


\section{LITERATURE REVIEW}

\subsection{Introduction}

The advancement of material along with the development of adaptive and intelligent structure systems has received increasing attention among structural engineers. Shape memory alloys (SMAs) are a class of smart materials that can exhibit a stable flag-shaped hysteretic behavior. They have been studied as promising materials to develop innovative devices and systems in structural engineering. Although nickel-titanium alloys were discovered initially in the 1960s and commercialized under the trade name Nitinol, the first use of SMAs in structural applications was initiated in the early 1990s as a new material for seismic isolation devices (Graesser and Cozzarelli, 1991). Since then, numerous researchers have conducted extensive investigations to explore the implementation of SMA-based systems for different structural applications. This section aims at providing a comprehensive review of shape memory alloys and the application of SMA-based devices, highlighting their characteristics, mechanical behavior, and the structural application of shape memory alloys in civil engineering.

\subsection{Shape Memory Alloy Characteristics}

\subsubsection{SMA Overview}

Shape memory alloys are a class of smart materials that have favorable characteristics for structural application, to improve the performance of structures, due to their unique properties. Two distinct properties of SMAs are shape memory effect (SME) and superelasticity (SE). These remarkable properties are related to phase transformations in crystal structures of SMA materials. The force required in these transformations can be either temperature-induced (shape memory effect) or stress-induced (superelasticity). The important crystal structures of SMAs are distinguished by their dual-phase microstructure: the austenite phase and the martensite phase. These beneficial characteristic and reduction in cost over the last decade of SMA production have attracted the attention of numerous researchers in scientific communities interested in developing seismic protection devices based on these properties. Although several compositions of SMA alloys have so far been developed, the most commonly used alloys in civil engineering include 
NiTi-based alloys, Copper ( $\mathrm{Cu}$ )-based based alloys, and Iron (Fe)-based alloys. The nickeltitanium (NiTi) alloys, also known as Nitinol, are the most widely exploited alloys for seismic applications, due to their superior material properties (DesRoches and Smith, 2004; Wilson and Wesoliwsky, 2005; Alaneme and Okotete, 2016). Recently, SMA cables made from NiTi wires, a relatively new class of structural elements, have been developed to provide significant cost advantages over a monolithic bar of equivalent diameter, while offering unique properties such as adaptive functionality (shape memory and superelasticity) and additional functionality (Reedlunn et al., 2013; Carboni et al., 2015; Ozbulut et al., 2015). Therefore, the development of the SMA cable can contribute to the extensive application of shape memory alloys in structural applications, due to the advantages of significant cost savings and unique adaptive characteristics.

\subsubsection{SMA Microstructure}

The unique properties of SMAs are associated with phase transformations between two distinct ordered atomic structures. The SMA microstructure exists in two stable phases, which have different crystal structures. The austenite phase is stable at high temperature and low stress with a body-centric atomic structure, while the martensite phase is stable at low temperatures and high stress with a parallelogram structure. The martensite structure can change its position by moving twin boundaries, while the austenite structure deforms by generation of dislocation. Consequently, the martensite phase is weaker and provides lower yield strength than that of the austenite phase. In addition, the martensite can exist in either of two crystal structures: twinned and de-twinned. Figure 2-1 illustrates different phases of shape memory alloy microstructure in 2D representation. The driving force for solid-to-solid phase transformations between these two phases can be induced either thermally or mechanically. The key effects of phase transformations between the austenite and the martensite assist in explaining the two fundamental characteristics of SMAs: superelasticity and the shape memory effect. These two behaviors depend on the four characteristic temperatures at which phase transformation occurs. 

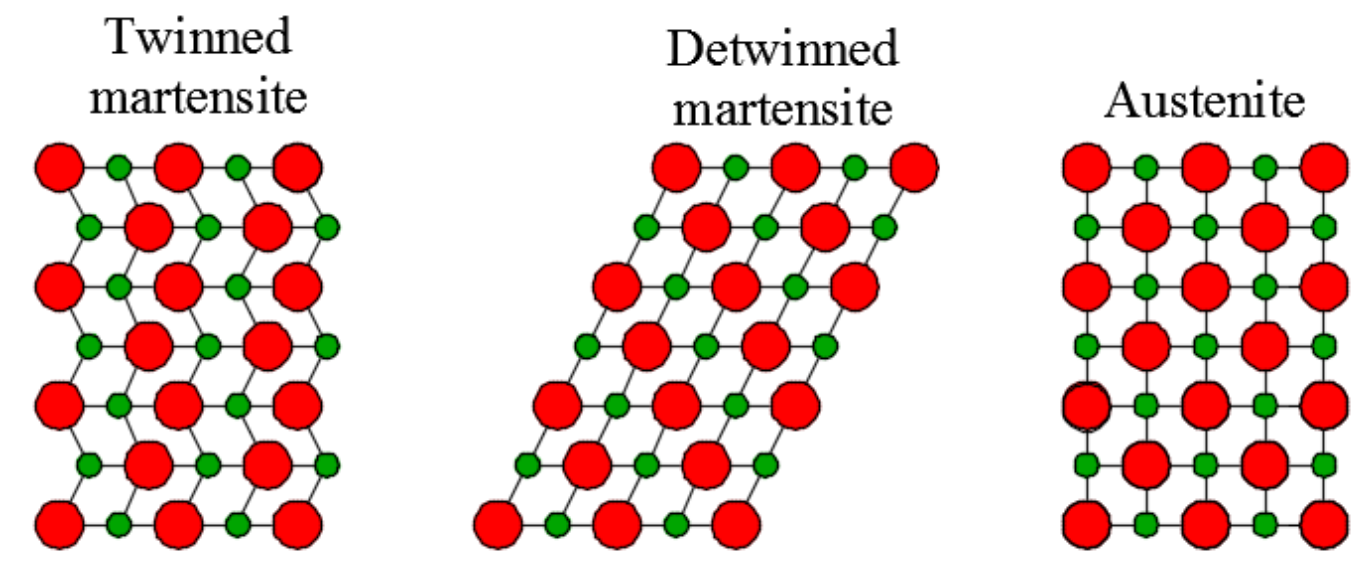

Figure 2-1 Different phases of shape memory alloy microstructure

In the stress-free state, the phase transformations of SMA materials can occur at four characteristic temperatures: martensite start temperature $\left(M_{s}\right)$ and martensite finish temperature $\left(M_{f}\right)$, austenite start temperature $\left(A_{s}\right)$ and austenite finish temperature $\left(A_{f}\right)$. Figure 2-2 highlights the relationship between the martensite fraction in an SMA material and the temperature. $M_{s}$ is the temperature at which the material begins transforming from the austenite to the twinned martensite while $M_{f}$ is the temperature at which the transformation is completed and the SMA material is exclusively in the martensitic phase. Similarly, $A_{s}$ is the temperature where the reverse transformation initiates from the twinned martensite to the austenite, and $A_{f}$ is the temperature at which the reverse phase transformation is completed to the austenitic phase.

\subsubsection{Shape Memory Effect}

Shape memory effect is a promising characteristic of SMAs that occurs when the material is deformed below $M_{f}$, recovering its original shape after being deformed through a thermal heating up to above $A_{f}$. Figure 2-3 illustrates the shape memory effect in relation to the temperature and deformation variation. In their low-temperature phase $\left(T<M_{f}\right)$, the SMAs show a phenomenon to indicate the shape memory effect, illustrating the build-up of residual stress fields. When SMA is cooled from its parent phase austenitic to the temperature below $M_{f}$, the SMA exhibits its twinned martensite phase through self-accommodation. This twined martensite phase can be easily changed 
by the application of external stress. After the application of external stress to the twinned martensite phase, SMA material reorients into the detwinned martensite phase in order to accommodate the resulting strain, if the applied stress is above the critical level. During the detwinning process of the martensite crystal structure, the stress remains almost constant until the material reaches the complete detwinned phase. Upon the release of the stress, the detwinned structure of the material remains in its deformed position. When the deformed material is heated to a temperature above $A_{f}$, the reverse phase transformation from the martensite to the austenite can occur and the material can regain its initial position, indicating complete shape recovery. Finally, the cooling of the SMA material causes the austenite crystal structure to transform into the low symmetry twinned orientation. This process is commonly known as the shape memory effect. Figure 2-4 indicates the stress-strain relationship of shape memory effect with corresponding phases.

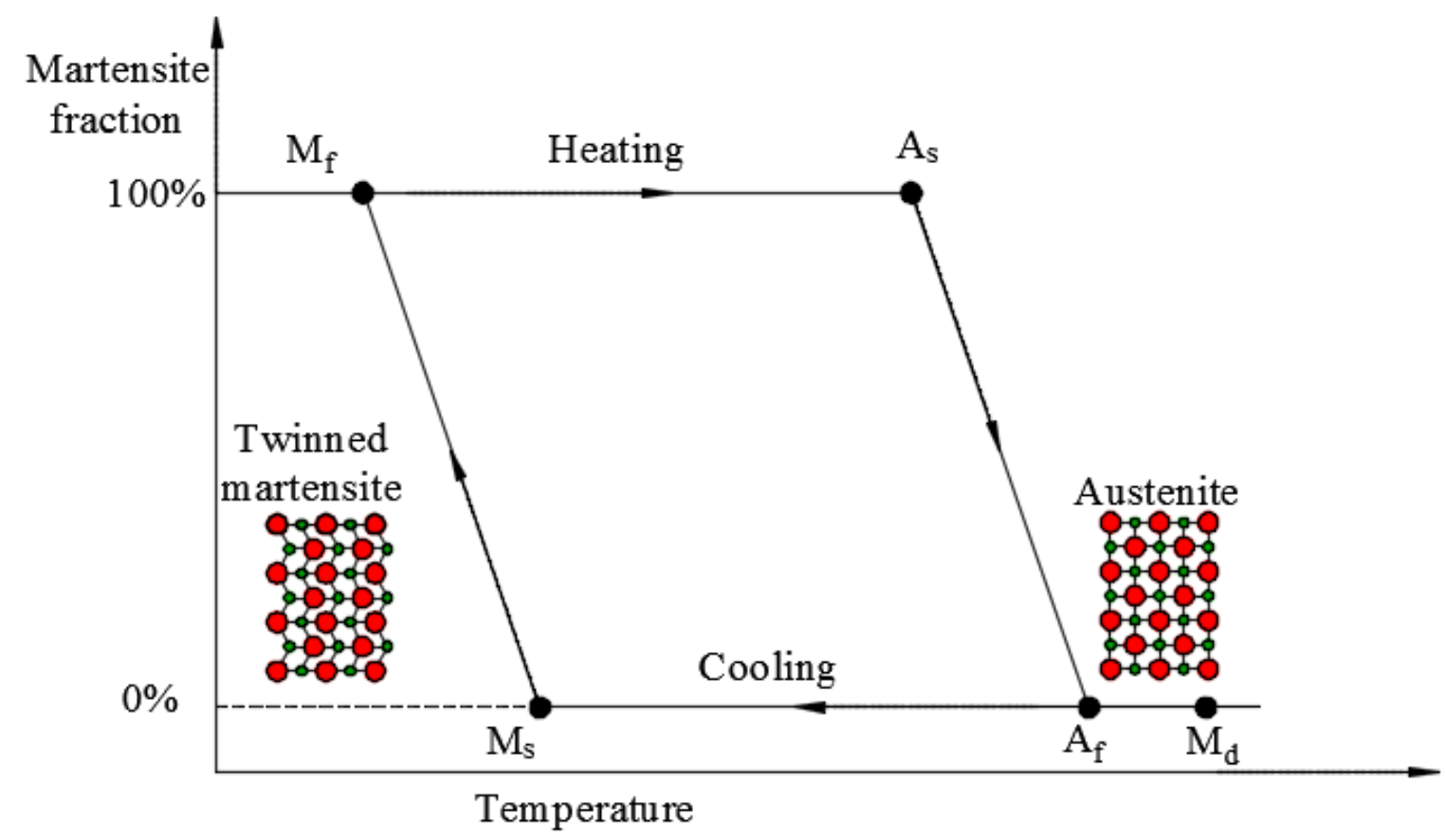

Figure 2-2 SMA microstructure representation of shape memory effect and superelasticity 


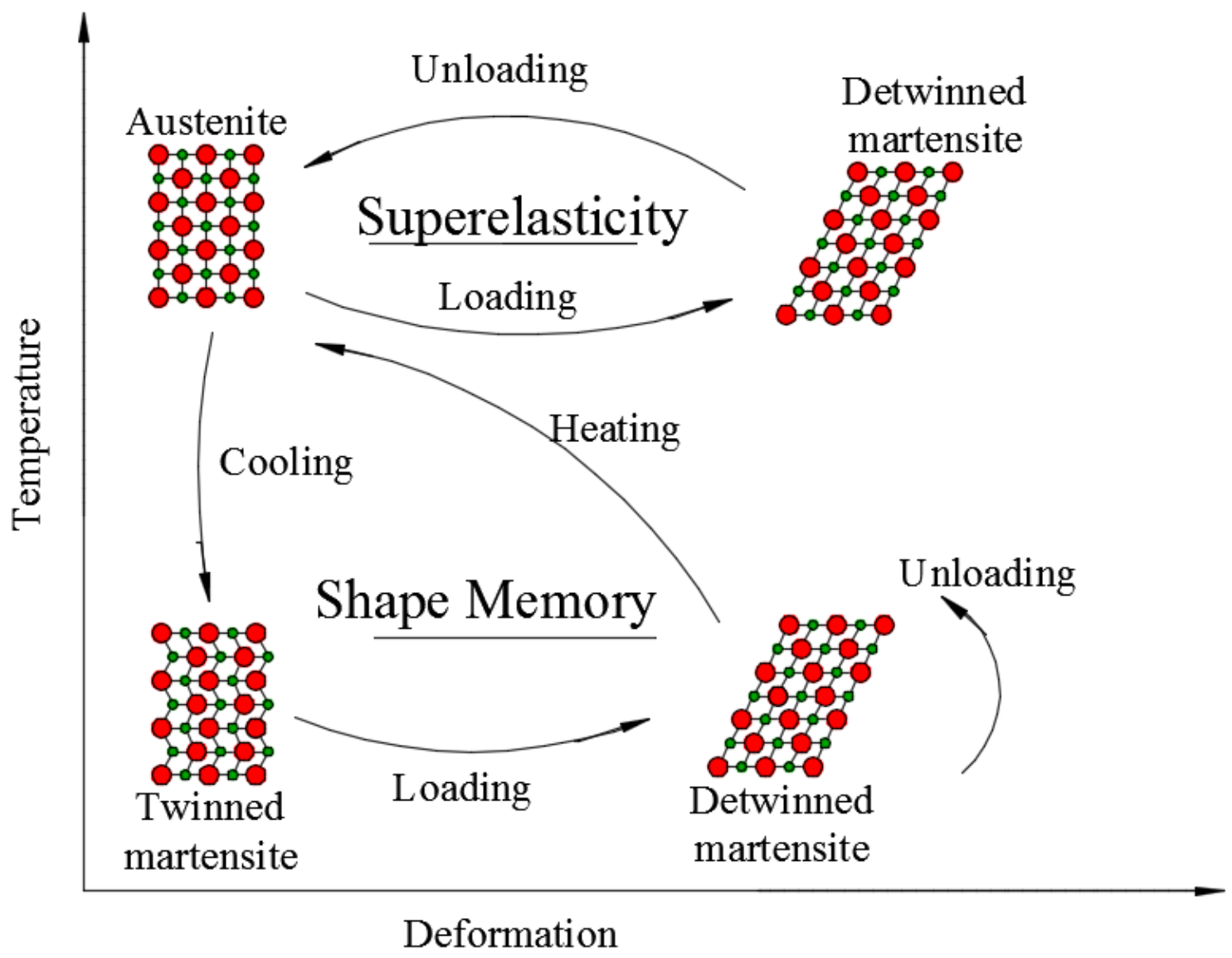

Figure 2-3 Temperature and deformation relationship of SMA microstructure for shape memory effect and superelasticity

\subsubsection{Superelastic Effect}

The superelasticity effect is another important feature of SMAs, where the material can recover large strain upon unloading if the temperature is above $A_{f}$. As shown in Figure 2-3, SMA exists in the austenite phase at high temperatures $\left(T>A_{f}\right)$. When the SMA material at the austenite phase is deformed at a temperature above $A_{f}$ due to the application of external stress, the SMA can transform to the detwinned martensite phase. Since this detwinned martensite phase is unstable, SMA transforms back to the austenite state and recovers its initial position when the external load is removed. This transformation process is called stress-induced martensitic transformation. Figure 
2-5 shows the superelastic behavior of SMAs under the loading-unloading process. This superelastic behavior of SMAs demonstrates considerable hysteretic loop with zero residual strain, indicating a promising self-centering mechanism, without the application of heat.

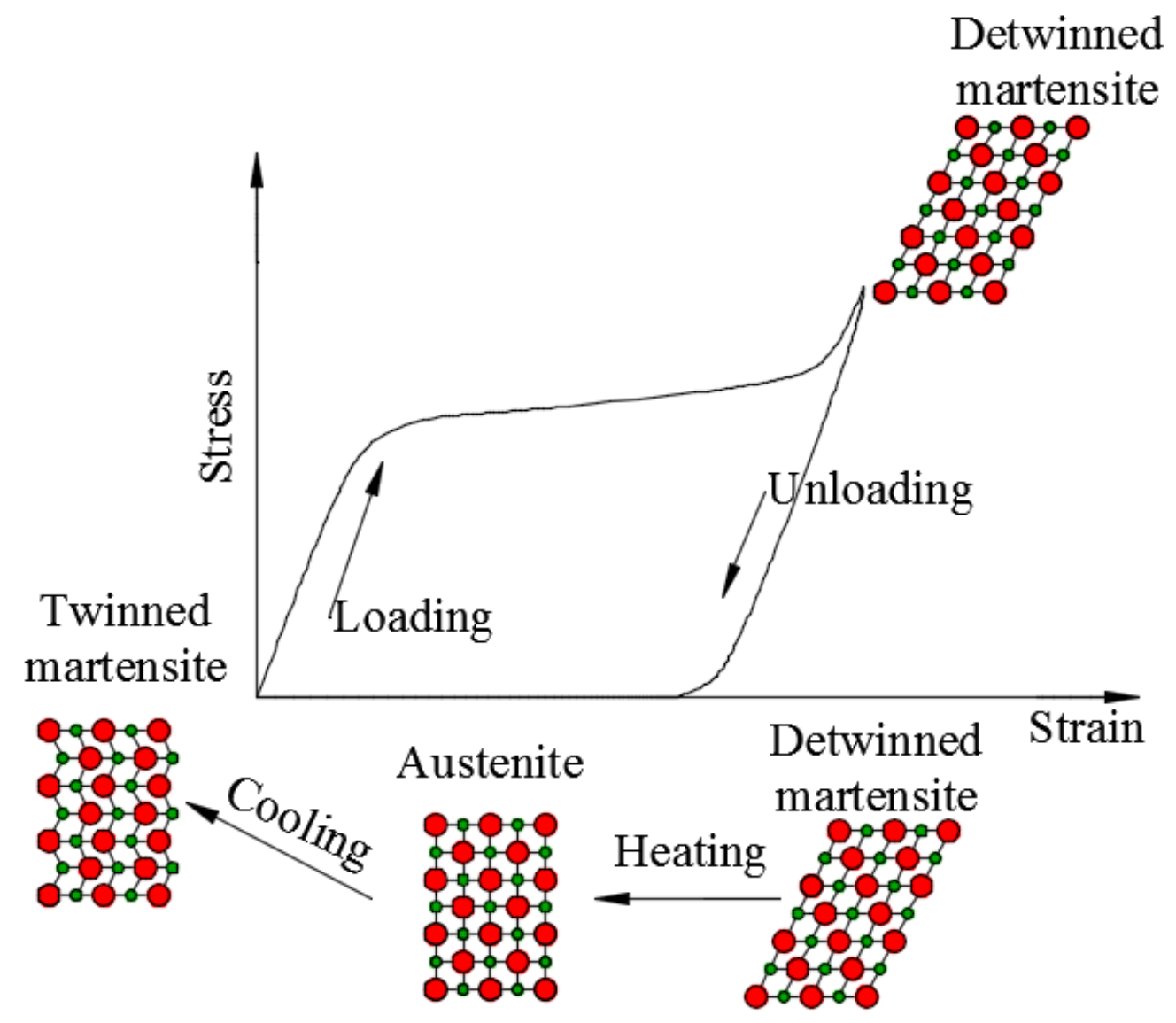

Figure 2-4 Stress-strain relationship for shape memory effect in SMAs

\subsubsection{Shape Memory Alloy Compositions}

Today, a wide range of SMA compositions has been developed and investigated to improve the characteristics of SMAs and the performance of structures in various engineering applications. The NiTi-based alloys have been the most commonly used and commercially available SMAs due to their superior thermo-mechanical properties, reliability, biocompatibility, and excellent strain 
recovery. In order to reduce the cost of SMA materials, researchers have developed different compositions of SMAs. The three major types of SMA systems that have been most explored over the last two decades are NiTi-based, copper $(\mathrm{Cu})$-based, and iron $(\mathrm{Fe})$-based SMAs.

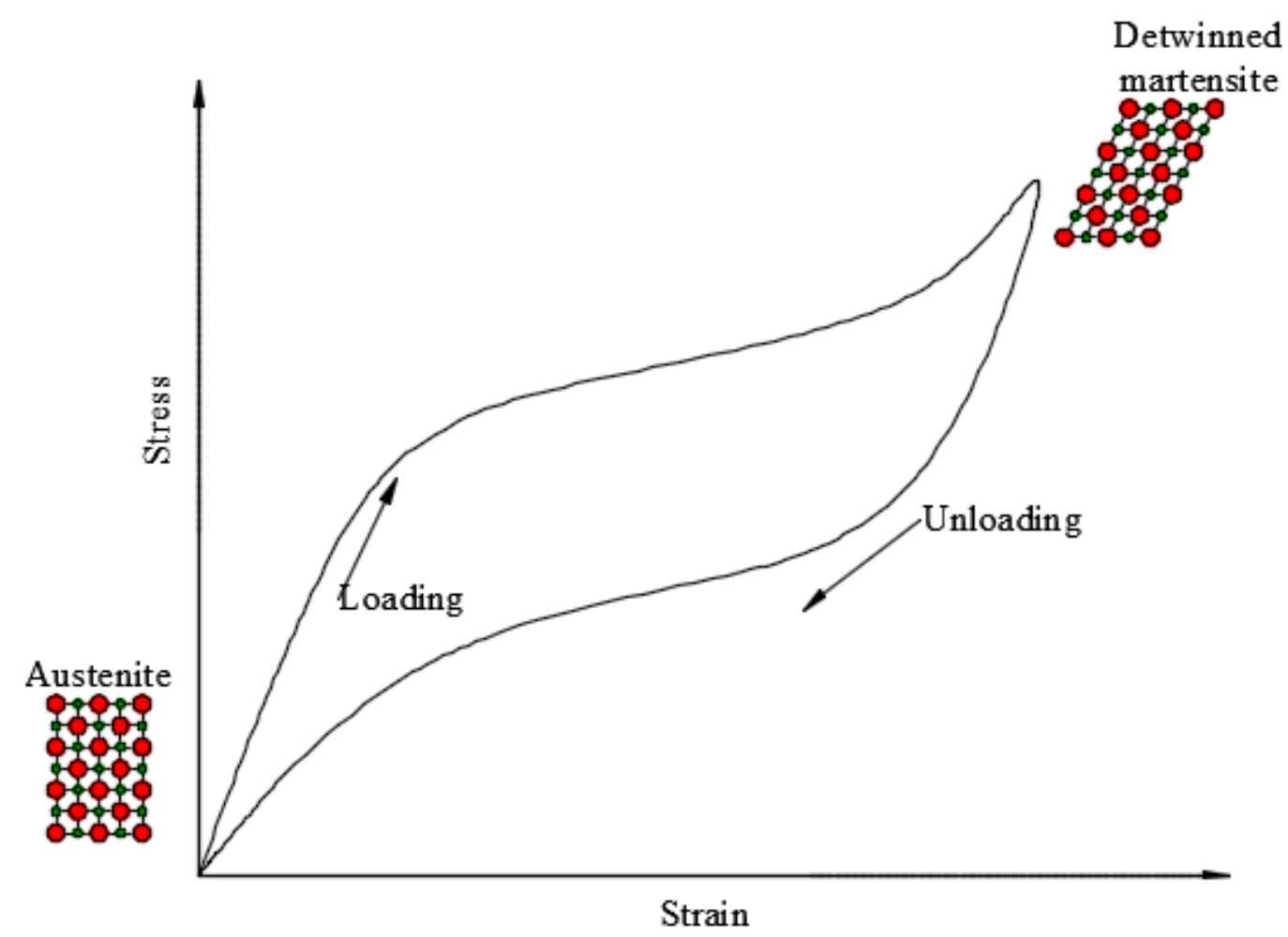

Figure 2-5 Stress-strain relationship for superelastic SMAs

\subsubsection{NiTi-based SMAs}

The nickel titanium (NiTi) alloys, alternatively recognized as Nitinol, have been extensively investigated and employed in different engineering applications, due to such strategic properties as large recovery strain, excellent superelasticity, and superior corrosion resistance. Three major advantages of NiTi SMAs are high stress capacity up to $700 \mathrm{MPa}$, recoverable stress of about $90 \%$ of the yield strength, and a large recovery strain of about $6-8 \%$. The distinct characteristics of NiTi derive from the presence of an almost equiatomic composition of nickel 
and titanium: $49-51$ atomic $\%$ of $\mathrm{Ni}$ to avoid undesirable phase transformation and decreasing transformation temperature. By adjusting their composition, NiTi alloys can be used in engineering structures for superelastic, shape memory, and damping applications at the ambient temperatures.

\subsubsection{Copper-based SMAs}

In an attempt to lower the cost of SMAs, numerous researchers have explored to produce $\mathrm{Cu}$-based SMAs for engineering applications. Although $\mathrm{Cu}$-based SMAs cannot provide excellent shape memory properties like NiTi SMAs, they have lower production costs and are easier to fabricate. Since $\mathrm{Cu}$-based SMA systems can be easily produced utilizing liquid metallurgy and power metallurgy routs, they are cost-effective. In addition, the $\mathrm{Cu}$-based SMAs exhibit lower strain rate effects and have larger operating temperature ranges compared to NiTi SMAs (Araki et al., 2012). The $C u$-based SMAs usually have lower recoverable strain and lower transformation stress than NiTi SMAs. The main $\mathrm{Cu}$-based SMAs include the binary alloys $\mathrm{Cu}-\mathrm{Zn}$ and $\mathrm{Cu}-\mathrm{Al}$. In these alloys, a third alloying element can be introduced to produce $C u$-based SMAs with desirable transformation temperature or microstructure. Several compositions of $\mathrm{Cu}$-based SMAs have been developed, such as $C u-Z n-A l, C u-A l-B e$, and $C u-A l-M n$.

\subsubsection{Iron-based SMAs}

The $\mathrm{Fe}$-based SMAs are recognized as the third predominant studied group of SMAs.. These SMAs are also called shape memory steel (SMS) and include $\mathrm{Fe}-\mathrm{Pd}, \mathrm{Fe}-\mathrm{Mn}$-Si, $\mathrm{Fe}-\mathrm{Mn}-\mathrm{Al}$, $\mathrm{Fe}-\mathrm{Ni}-\mathrm{C}$, and $\mathrm{Fe}-\mathrm{Ni}-\mathrm{Co}-\mathrm{Ti}$. These SMA compositions have been produced in an attempt to lower the cost and study their suitability for large-scale engineering applications. For example, Tanaka et al. (2010) introduced a $\mathrm{Fe}-\mathrm{Ni}-\mathrm{Co}-\mathrm{Al}-\mathrm{Ta}-\mathrm{B}$ SMA indicating superelastic strain of about $13 \%$ and very high tensile stress. Similarly, Omori et al. (2011) developed $\mathrm{Fe}-\mathrm{Mn}$-Al-Ni showing superelastic strain over $6 \%$, which revealed good superelastic behavior at room temperature. Because these Fe-based SMAs have been developed to conduct laboratory testing for research only, they have not yet been made commercially available.

\subsubsection{Comparison of Different Compositions in SMAs}


The comparison of the modulus of elasticity and recovery strain of three SMA systems is illustrated in Figure 2-6. It can be seen that $C u$-based SMAs have lower modulus of elasticity compared to the other two SMA systems. The Fe-Ni-Co-Al-Ta-B SMAs show very high recovery strain, while $\mathrm{Fe}-\mathrm{Mn}-\mathrm{Al}-\mathrm{Ni}$ exhibits the relatively high modulus of elasticity. Table 2-1 summarizes the comparative advantages of three major SMA systems: NiTi-based, $\mathrm{Cu}$-based, and $\mathrm{Fe}$-based SMA.

Table 2-1 Comparative advantages of three shape memory alloy compositions

\begin{tabular}{cccc}
\hline Property & NiTi-based & $\boldsymbol{C u}$-based & $\boldsymbol{F e}$-based \\
\hline Modulus of Elasticity & Moderate & Low & High \\
Shape memory effect & High & Moderate & Low \\
Maximum recoverable strain & $8 \%$ & $5 \%$ & $<5 \%$ \\
Production cost & High & Low & Low \\
Fabrication & Low & Good & Moderate \\
Workability & Moderate & Low & Good \\
Processing & Demanding & Easy & Easy \\
\hline
\end{tabular}

\subsection{Mechanical Behavior of NiTi Shape Memory Alloys}

The superelastic behavior of SMAs has received the attention of researchers for civil engineering applications over the last two decades. Particularly, NiTi SMAs have been the most popular SMAs, indicating promising characteristics for seismic applications, such as large strain recovery; high strength with stable hysteresis behavior; excellent fatigue and corrosion resistance; and significant energy dissipation capacity (Kim et al., 2002). The mechanical characteristics of the NiTi SMAs are influenced by various parameters such as cyclic loading, strain rate, temperature, and thermomechanical treatments. Hence, this section presents the effect of these parameters on the mechanical behavior of the NiTi SMAs. In past decades, many research studies have investigated the mechanical behavior of superelastic NiTi SMAs through experimental 
testing. The following subsections provide the review of the influence of cyclic loading, strain rate, and temperature on the hysteretic behavior of the NiTi SMAs, in addition to the general characteristics of NiTi SMAs.

\subsubsection{General Characteristics of NiTi SMAs}

A number of researchers have conducted experimental testing to explore the mechanical behavior of NiTi SMAs in different shapes and forms, such as bars, wires, or cables. However, there have been more studies on SMA wires than any other form of SMAs. That can be attributed to the easier manufacturing process and lower cost of SMA wires. In addition, most of the previous studies indicated that the SMA wires have better superelastic characteristics compared to SMA bars (Dolce and Marnetto, 1999; MANSIDE, 1998). DesRoches et al. (2004) investigated the

cyclic behavior of both SMA bars and wires. They revealed that SMA wires and bars with a variation in diameter from $1.8 \mathrm{~mm}$ to $25.4 \mathrm{~mm}$ exhibited satisfactory re-centering capabilities; however, SMA wires showed higher strength and damping capacity compared to the larger SMA bars. Other researchers demonstrated that both bars and wires can provide good superelastic properties by adopting suitable chemical composition, deformation processing, and heat treatment (Tyber et al., 2007; McCormick et al, 2007). 

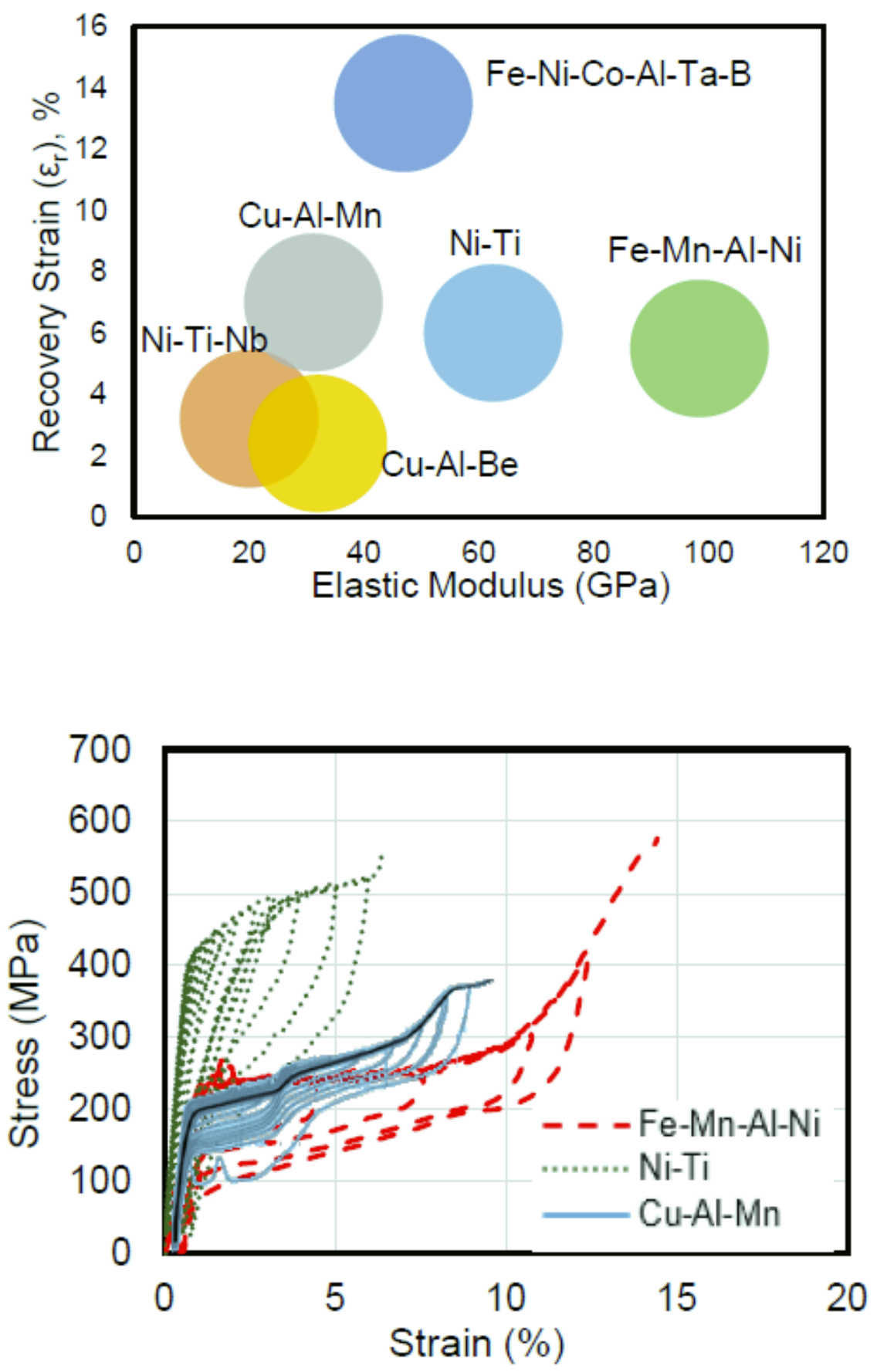

Figure 2-6 (a) Comparison of elastic modulus and recovery strain and (b) comparison of hysteretic response of different SMAs (Billah AHMM, 2015) 
Damping capacity represents the ability of a material to absorb or release the vibrational energy of a structure by converting the mechanical energy into heat energy. The damping capacity of SMAs is related to the hysteretic movement of the martensite variant interfaces (Humbeeck, 2003; San Juan and No, 2003; Cai et al., 2005; Chen et al., 2009). The equivalent viscous damping ratio of SMA material can be calculated as a function of cyclic strain level. One study investigating the damping capacity of SMA materials reported that the equivalent viscous damping attained its maximum value at 4-5\% strain level and decreased after that strain level (DesRoches et al., 2004). The study also indicated that the equivalent viscous damping of SMAs wires is higher compared to SMA bars, with a damping ratio of $4-8 \%$ for wires and $2-4 \%$ for bars. Furthermore, the results showed that the damping capacity is inversely proportional to the bar diameter: the lower the bar diameter, the higher its damping capacity. In another study of the superelastic NiTi bar and wires, the NiTi wires demonstrated a higher equivalent viscous damping of $5.3 \%$ for the $0.254 \mathrm{~mm}$ diameter wires, while the NiTi bars of $12.7 \mathrm{~mm}$ diameter showed a $2.7 \%$ equivalent viscous damping under the quasi-static tension loading (McCormick et al., 2006). In another study, it was reported that the equivalent viscous damping of a $12.7 \mathrm{~mm}$ diameter SMA bar was approximately $30 \%$ higher than that of a $19.1 \mathrm{~mm}$ diameter bar (McCormick et al., 2007). It was also shown that residual displacements slightly increased with larger diameter SMA bars.

\subsubsection{Cyclic Properties}

Earthquake events induce cyclic deformations. To comprehend the cyclic behavior of NiTi SMAs under seismic events, numerous researchers have conducted experimental tests to characterize the cyclic behavior of SMAs under repeated cyclic loading with incremental strain levels. In order to investigate the influence of repeated loading and fatigue, some researchers have tested superelastic SMA bars and wires under cyclic loading conditions. The test results showed that the residual strain increases while the loading plateau stress level and hysteric loops decrease with an increasing number of loading cycles (DesRoches et al., 2004; Dolce and Cardone, 2001; Gong et al., 2002). After recognizing the variations in the forward phase transformation stress level, some researchers investigated the influence of training cycles on NiTi elements to achieve stabilization in the hysteric behavior. Particularly, applying training loading cycles (approximately 20 cycles) on NiTi elements provides a stable flag-shaped hysteric behavior with enhanced recentering capabilities (MANIDE, 1998; McCormick and DesRoches, 2006; Wang et al., 2003). 
Interestingly, the maximum variation on the cyclic behavior was observed between the first and second cycles, which was caused by small levels of localized slip (DesRoches et al., 2004). Figure 2-7 displays the cyclic tensile test result of NiTi wires having $2 \mathrm{~mm}$ diameter, indicating the stressstrain curve with the variation in cyclic behavior in forward transformation (Malecot et al., 2006).

McCormick et al. (2007) presented experimental test results of the large-diameter NiTi bars of 12.7, 19.1, and $31.8 \mathrm{~mm}$, while Wang et al. (2016) demonstrated the real-scale test results of large size superelastic SMA bars with diameter varying from $8 \mathrm{~mm}$ to $30 \mathrm{~mm}$. Similarly, DesRoches et al. (2004) reported the cyclic properties of superelastic SMA bars and wires having varying sizes, from $1.8 \mathrm{~mm}$ wires to $25.4 \mathrm{~mm}$ diameter, to demonstrate the influence of bar size and loading history on the behavior and properties of superelastic SMAs. Furthermore, other researches also reported the fundamental cyclic behavior of superelastic NiTi wires (Parulekar et al., 2014; Zhang and Zhu, 2007; Dezfuli and Alam, 2013; Dolce and Cardone, 2001). These test results showed that the hysteresis loops of both SMA wires and bars experience similar degradation under cyclic load.

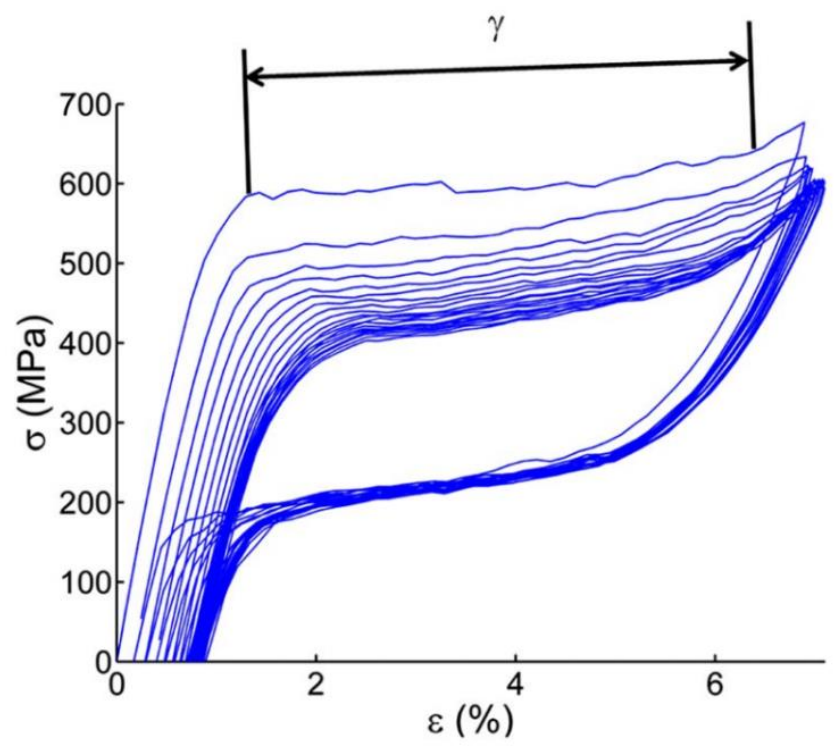

Figure 2-7 Results of cyclic tensile tests on NiTi wires (Malecot et al., 2006). 
Recently, researchers have reported experimental test results of SMA cables, relatively new structural elements that demonstrate numerous desirable qualities, superior performance than those of large-section bars, and adaptive functionality. These structural cables are developed by assembling several strands laid helically around a central core. The strands also consist of several small-diameter wires helically wrapped around a central wire. The SMA cables offer unique properties, such as shape memory or superelasticity, and new adaptive functionality (Reedlunn et al., 2013; Carboni et al., 2015; Ozbulut et al., 2015). Additionally, SMA cables generally exhibit many of the advantages of classical wire cables and leverage the excellent mechanical properties of thin SMA wires in developing large force tension elements. The SMA wires generally contribute relatively large tensile stiffness and strength with design flexibility. Next, they are relatively damage-resistant and fatigue-resistant, since the failure of a single wire in a multi-wire cable provides redundancy, unlike the sudden fracture in a large-diameter monolithic bar. Furthermore, the cost comparison between SMA wire and a monolithic large-diameter bar of comparable size demonstrated that the SMA cables with superior characteristics result in considerable economical advantage over a monolithic bar of comparable size (Reedlunn et al., 2013). By adopting the recently developed, highly optimized manufacturing process for wires, SMA cables can expedite the application of SMAs in infrastructure applications due to their superior mechanical properties, large force capacities, and lower cost over SMA bars. Figure 2-8 shows the hysteric cyclic behavior of a single NiTi wire and the SMA strand with the seven-wire NiTi, exhibiting a restoring stress of the strand lower than the single wire at the same strain level (Reedlunn et al., 2013). The lower stress in the strand caused by the intertwined sliding friction forces generated between the wires. 

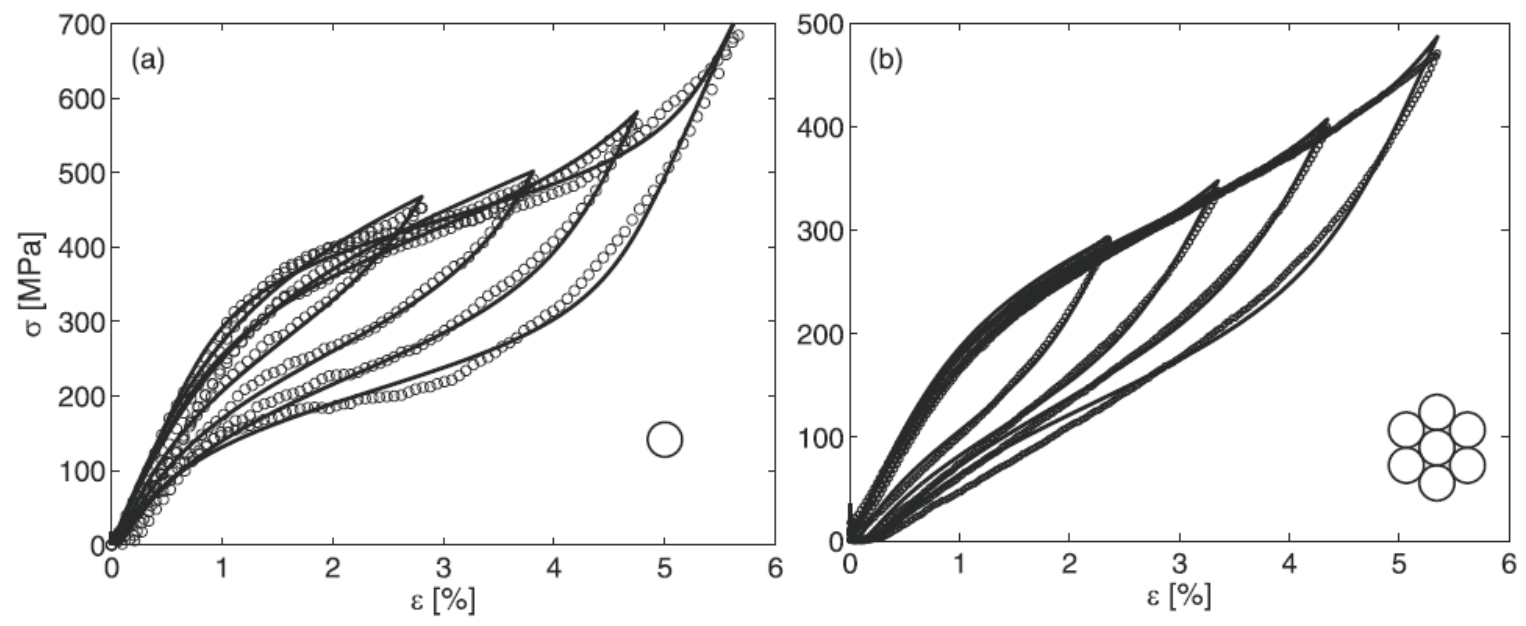

Figure 2-8 Hysteresis cycles of stress-strain curves: (a) response curves of the single Nitinol wire; (b) response of the seven-wire Nitinol strand (Reedlunn et al., 2013)

\subsubsection{Influence of Strain Rate}

The mechanical behavior of NiTi SMAs is significantly influenced by their loading rate. The rate-dependent behavior is influenced by the complex interaction between stress, temperature, and the rate of heat generation during phase transformation (Azadi et al., 2006). Numerous experimental test results demonstrated that the generation or absorption of the latent heat in the forward and reverse transformations occurs during the martensitic phase transformations (He and Rong, 2004; Liu and Huang, 2006). The test results revealed that the SMA material requires adequate time to exchange phase transformation-induced latent heat to the environment. However, insufficient time is available during the loading with high strain rates to transfer the heat in that process. Therefore, the temperature of the SMA material changes during its forward transformation and alters the shape of the hysteresis loops and transformation stresses.

The rate of loading influences the mechanical response characteristics of NiTi SMA, as observed in civil engineering structures under the dominant frequency range of $0.2 \mathrm{~Hz}$ to $4.0 \mathrm{~Hz}$ during an earthquake event. The increase in the loading frequency, and consequently the strain rate, increases the loading plateau and decreases the amount of the hysteretic damping of NiTi SMA (DesRoches et al., 2004; Dolce and Cardone, 2001). Wu et al. (1996) reported that the strain 
rate effects are caused by the inability of SMA to dissipate heat energy. The larger diameter SMA bars generally display greater dependence on strain rate.

The loading effect on the superelastic behavior of NiTi SMA is an important consideration in evaluating its mechanical characteristics before the actual implementation in civil engineering, since the SMA material encounters dynamic effects during earthquake events. Past research studies reported an inconsistency in findings on the effect of loading rate, which can be caused by multiple factors, such as the different composition of the material, the experimental testing at different strain rates, and the experimental test conditions. When strain rates increase, the reverse transformation stress is also increased, without the considerable variation in the forward transformation stress, but the energy dissipation is decreased, as reported by Wolons et al. (1998) and Ren et al. (2007). Another study showed that the increase in strain rates contributes to an increase in both the forward and reverse stresses. Furthermore, Tobushi et al. (1998) noticed that the higher strain rates attribute to increase in the forward transformation stress, but decrease in the reverse transformation stress.

\subsubsection{Influence of Temperature}

A temperature variation can considerably influence the material performance and hysteretic behavior due to thermal changes during the cyclic loading, since the phase transformation and superelastic behavior of NiTi wires are also dependent on temperature variation. A number of researchers have conducted experimental tests to determine the influence of the temperature variation on the superelastic behavior of SMAs (Dolce and Cardone, 2001; Chen and Song, 2006; Churchill et al., 2009; Yin and Sun, 2012). The critical stress that activates the phase transformation changes significantly with temperature variations, as reported in the literature. It was reported that the increase in temperature linearly increases in the transformation stress and linearly decreases the equivalent viscous damping. Figure 2-9 shows the stress-strain curves at various temperatures of superelastic NiTi wires experimentally tested by Chang et al. (2006). It is reported that the superelastic behavior of NiTi wires was observed when the NiTi wires were heated above $0^{\circ} \mathrm{C}$, and the residual strains were noticed in hysteric responses above $40^{\circ} \mathrm{C}$. It can be seen in the figure that the hysteresis curves can shift upward as the temperature increases. 


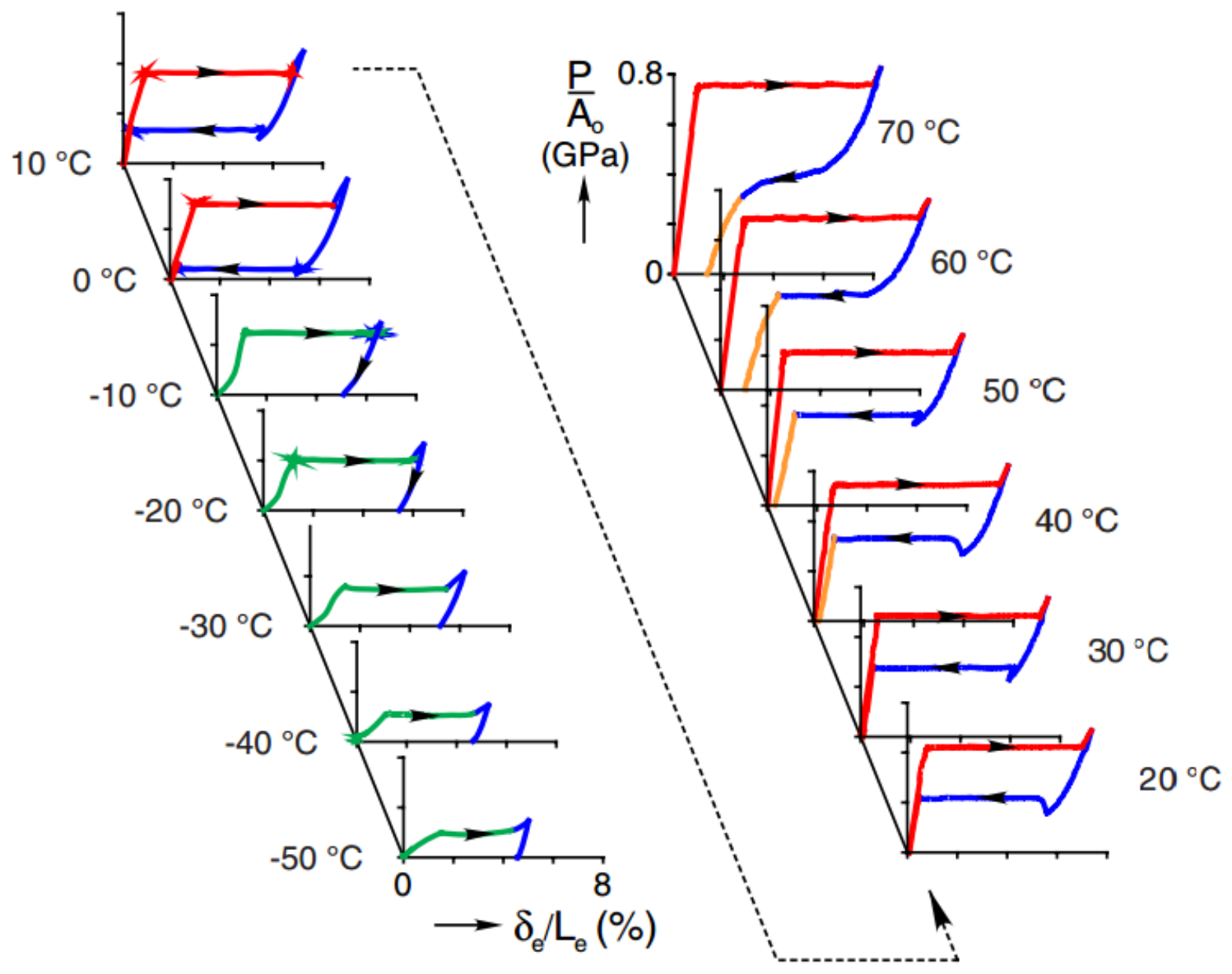

Figure 2-9 Stress-strain curves of NiTi wires at different temperatures (Churchill et al., 2009)

\subsection{Applications of Shape Memory Alloys in Civil Structures}

The SMAs have been explored for various civil engineering applications. In this section, the applications of SMAs in steel beam-column connections, steel bracing systems, isolation systems and passive control devices are reviewed. 


\subsubsection{SMA-based Structural Connections}

Numerous researchers have investigated the potential application of SMAs as a structural beam-column connection element that can ultimately control the damage and structural responses in both structural connections and the main structural components.

Leon et al. (2001) made two full-scale experimental tests on exterior joint connections using Nitinol SMAs according to the SAC loading protocol, to evaluate their connection behavior considering the ability of SMA tendons. As shown in Figure 2-10, the SMA tendons in a semirigid connection were assembled from the flange of the beam to the column to serve as the primary load transforming element for the steel structural connection. The SMA tendons were designed to initiate entirely the martensitic behavior, and subjected to several loading cycles at higher cyclic strains, up to $4 \%$, without damage. To activate the shape memory behavior of the tendons, they were heated above the transformation temperature upon the end of the cyclic loading to regain their original configuration.

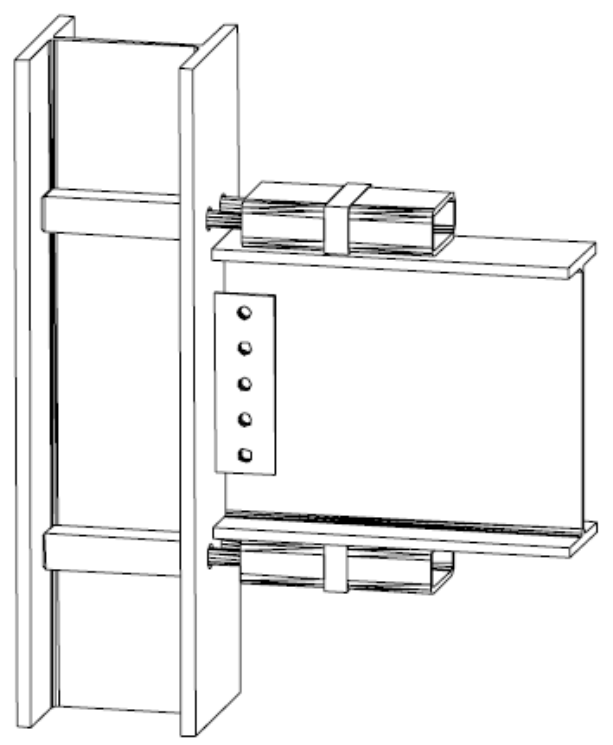

Figure 2-10 Innovative Steel Beam-Column Connection Using Shape Memory Alloys Tendons (Leon et al., 2001)

For comparison purposes, the companion steel connections were also experimentally tested to evaluate their performance. It was observed that the SMA connection revealed the stable and 
repeatable hysteresis behavior for rotations up to $4 \%$. Comparing the hysteric behavior of the first and second test series, the moment rotation characteristics of the SMA tendon in the second testing series were identical to the first test. This result indicated the ability of SMA-based structural connections to sustain repeated large deformations without strength degradation, and to recover their initial properties following heating above their transformation temperature.

Ma et al. (2007) investigated the feasibility of an innovative beam-column connection consisting of an extended end plate with SMA bolts, beam flange ribs, and web stiffeners to predict the behavior of the SMA connection under quasi-static tests. Advanced 3D finite element models of the conventional end-plate bolted connection and the SMA-based structural connections were developed to compare the behavior of these two. This new SMA-based beam-column design concept appeared to enhance the seismic performance of structural connections, due to its excellent ductility and energy dissipation demands accommodated by the deformations of the superelastic SMA bolts. The result indicated that the SMA connection achieved the desired deformation capacity by controlling the moment-carrying capacity of the bolt cluster below the elastic flexural capacity of the connecting beam and forming the plastic hinge within the beam-column interface. Similarly, SMA connections recovered up to $94 \%$ of the total deformation due to the nonoccurrence of local buckling of SMA connections during entire loading cycles, indicating the effectiveness of increasing the distance of the bolt to reduce residual deformation. Hence, the resulting benefits of SMA connections were enhanced ductility, moderate energy dissipation capacity, and excellent re-centering capability, which can minimize post-earthquake repair work and costs to repair structural elements. Figure 2-11 illustrates the SMA connection diagram in the beam-column connection. 


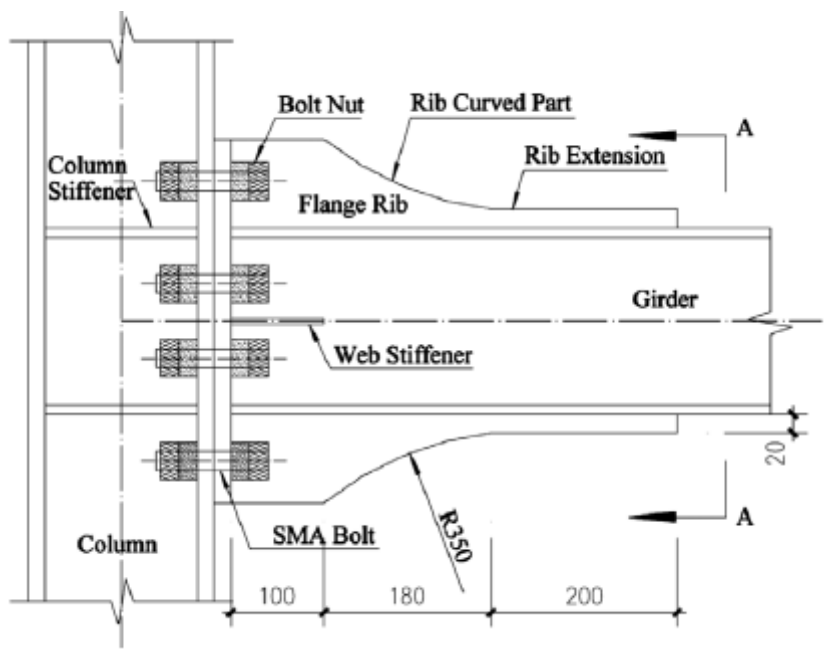

A-A

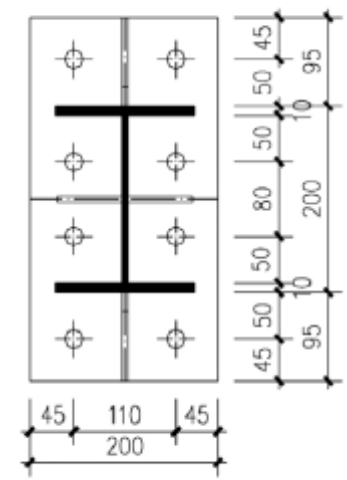

Figure 2-11 The SMA connection diagram (Ma et al., 2007)

Rofooei and Farzaneh (2011) numerically studied the seismic behavior of superelastic SMAs in the beam-column connections of steel moment resisting frames. The numerical simulations were conducted for a set of steel structural models with a different number of stories and varying eccentricities, incorporating SMA connections subjected to different bi-directional earthquake components. In the study, extensive nonlinear time history analyses were made in order to examine the performance of SMA-equipped structural connections in reducing their seismic response, and to evaluate the energy dissipation capability of the structural connection. The comparative evaluation of inter-story drift, base shear capacity, moment-rotation behavior of structural connection, and residual strains was reported for both the conventional steel moment resisting frame and the frame model with SMA connections, to demonstrate the efficiency of the steel structural model equipped with new SMA connections. The result indicated that the application of the SMA connection in the steel structural model reduced the base shear force of the structural model and residual displacement due to the superelastic characteristic of SMA materials. However, structural models with SMA connections were not able to produce significant reduction in lateral displacements, compared to the responses of the structural model with fixed connection models. 
Sepulveda (2008) reported the experimental investigation of steel structural connections with copper-based SMA bars for a prototype of partially restrained connections subjected to a controlled cyclic displacement history. The physical model of the structural connection included an end-plate connection at a rectangular hollow structural beam connected to a wide flange column, which were connected by four $\mathrm{CuAlBe}$ SMA bars in the austenitic phase. A simple experimental test set-up for the SMA-based structural connection was developed to explore the potential of SMA prestressed bars subjected to dynamic cyclic tests. The static tensile test results showed that the fracture strain was approximately $8 \%$, with a transgranular fracture mechanism. Similarly, SMA-based structural connections demonstrated a moderate level of energy dissipation, superelastic behavior, and no strength degradation, up to $3 \%$ drifts under several cycles of dynamic loading. After evaluating the performance of structural connections, numerical simulations of a three-story benchmark building were also presented, to compare the dynamic performance of a rigid steel building and a partially restrained building equipped with SMA-based connections. The preliminary results highlighted that copper-based SMA rods at structural connections did not provide a specific conclusion about the potential advantage of $C u A l B e$ connections in steel moment resisting frames.

Speicher et al (2011) explored a half-scale interior beam-column connection, as shown in Figure 2-12, incorporating superelastic nickel-titanium (NiTi) shape memory alloy (SMA) tendons, which were designed, fabricated, and tested experimentally. To assess the feasibility of structural connections in a moment resisting frame, experimental tests were conducted in frames designed with the four different tendons, for structural connections made of (i) steel, (ii) martensitic NiTi SMA, (iii) superelastic NiTi SMA, and (iv) superelastic NiTi SMA in parallel with aluminum. The low-strength aluminum bars were employed to contribute additional energy dissipation. In this study, the re-centering connections were designed in such a way that inelastic deformations occurred in the tendons, while the other members of the structural system remained in the elastic range. The experimental test results illustrated that a superelastic NiTi SMA-based structural connection contributed excellent ductility, energy dissipation, and re-centering properties compared to the other two connections made from steel and martensitic NiTi SMA. The superelastic SMA-connection was found to possess significant superelastic behavior at drift levels below $1 \%$, and to recover $85 \%$ of its total deformation after being cycled to $5 \%$ drift demand, 
highlighting the excellent re-centering capabilities of the superelastic nickel-titanium SMA-based beam-column connections.
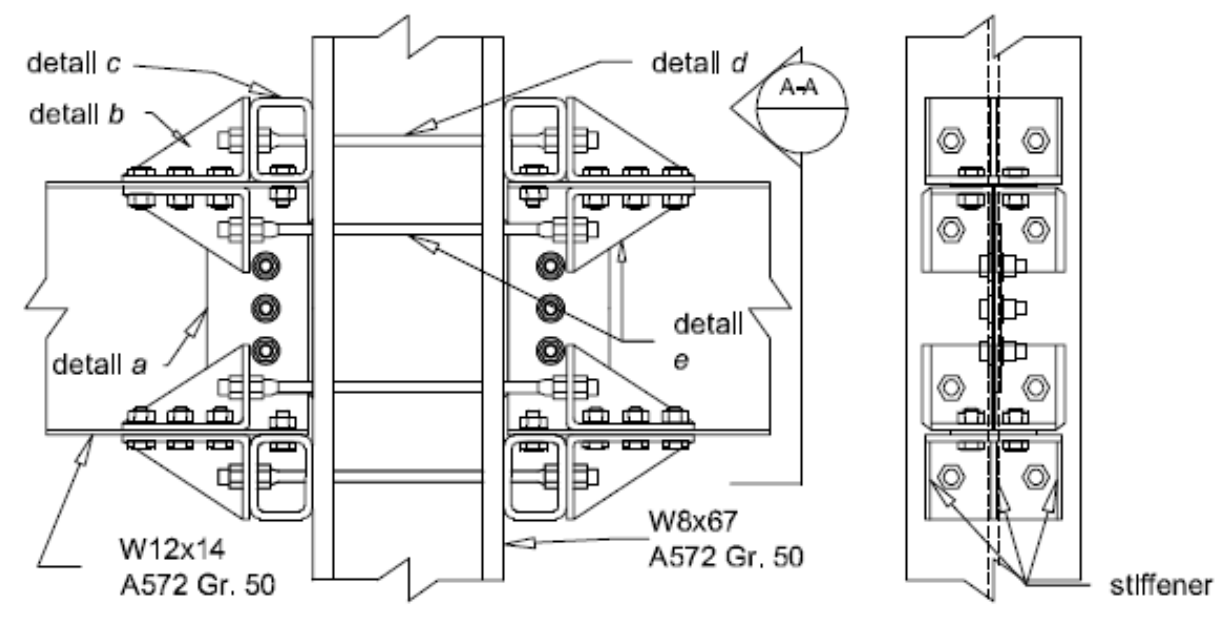

Figure 2-12 Steel beam-column connection details (Speicher et al., 2011)

Yam et al. (2015) conducted both numerical study and experimental tests on the extended end-plate connections with NiTi SMA bolts, as shown in Figure 2-13, to demonstrate responses in terms of re-centering ability, supplementary energy dissipation, and hysteretic stability. Initially, seven full-scale tests were performed to validate the cyclic responses of numerical modeling results. The results of detailed finite element analyses demonstrated good agreement with the test results in terms of the hysteric moment-plastic rotation behavior, energy dissipation characteristics, and re-centering ability. After validating the numerical modeling strategy, a parametric study was carried out to demonstrate the influence of bolt geometry/layout; beam behavior; beam-toconnection strength ratio; end-plate thickness; column deformation behavior; and shear-resisting mechanism. In order to address the major concerns identified from the parametric study, an improved connection type was proposed, using a high-strength (HS) SMA hybrid solution, in which HS bolts and SMA bolts were employed to resist the shear force and to provide recoverable bending resistance, respectively. The hybrid structural connection incorporated the multifunctional use of SMA Belleville washers for protecting the HS bolts, enabling connection re- 
centering ability, and offering supplementary energy dissipation. Finally, the feasibility of the HSSMA hybrid connection was confirmed through a detailed FE model, and a design recommendation on the NiTi SMA equipped end-plate connection was proposed for the normal design of such connections.

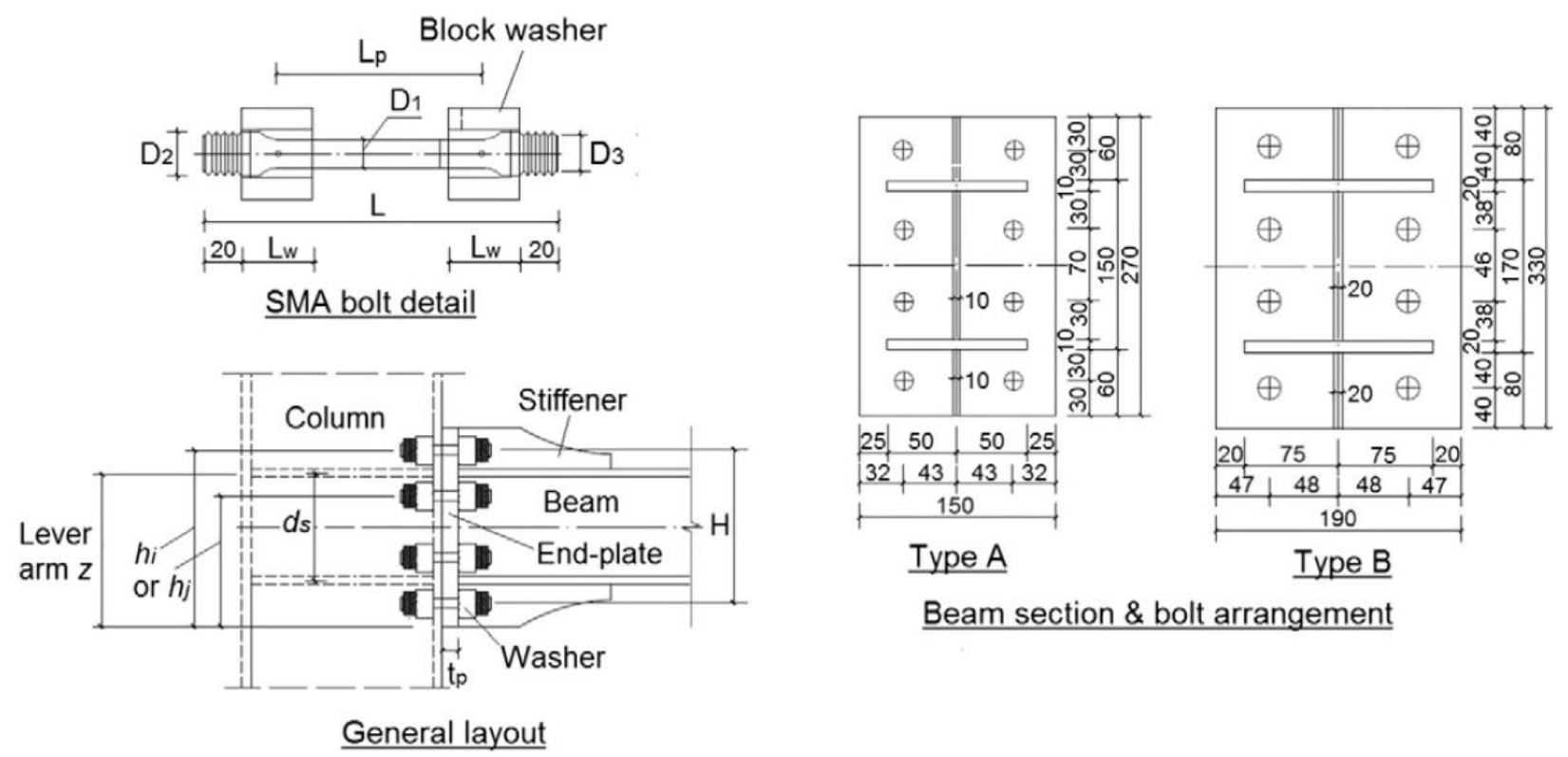

Figure 2-13 Geometric configurations and layouts (Yam et al., 2015)

\subsubsection{SMA-based Bracing Systems}

McCormick et al. (2007) investigated the seismic performance of concentrically braced frames with superelastic SMA braces, to address the limitations of the conventional brace frame systems in terms of ductility and energy dissipation due to the buckling of conventional braces. In order to reduce the length of SMA braces, chevron braced frames were developed employing SMA bars in parallel, consisting of each single bar, with varying diameters from 12.7 to $31.75 \mathrm{~mm}$, connected to the frames through rigid elements. An extensive analysis of three- and six-story concentrically braced frame systems with either superelastic SMA braces or conventional steel braces was conducted. A series of nonlinear time history analyses were conducted to identify the potential benefits of using SMA braces for the dynamic control of braced-frame systems. For the comparative evaluation of two braced systems, the frames with SMA braces were designed to 
provide yield force and initial stiffness to equal that of the conventional steel brace system, such that both frame systems revealed the same fundamental period. The results highlighted that frames with SMA braces contributed promising benefits in controlling inter-story drift and residual drift during an earthquake event, indicating excellent re-centering characteristics of superelastic SMAs. Furthermore, SMA braces appeared to be the most effective in limiting inter-story drift in the lower stories of tall buildings, compared to the drift of conventional steel brace systems.

Asgarian and Moradi (2011) studied the seismic performance of steel frame systems installed with superelastic SMA braces in buildings having various stories and different bracing configurations, to evaluate the efficiency of SMA braces. In order to compare the responses, steel buildings with varying stories $(4,6,8,10,12$, and 14 stories) for four different bracing configurations (diagonal, split $\mathrm{X}$, chevron $\mathrm{V}$, and inverted V) were designed, considering three groups of braced frames: an SMA brace system with rigid element, an SMA brace system with buckling restrained braced (BRB) element, and a BRB system. To evaluate the potential benefit of an SMA-based innovated bracing system, the dynamic response of a steel braced frame equipped with SMA braces was compared to the brace system with BRBs, subjected to three ground motion records. The results of the comparative study revealed that the frame system with SMA braces provided a more effective method to reduce both inter-story drift and residual drift, and to minimize losses associated with damage to structural systems during earthquake events, independent of both the structures' brace configurations and their stories. Moreover, the results indicated that the energy dissipation capabilities of the SMA-braced systems were comparable to BRB frame systems. In addition, the SMA-braced systems were able to reduce peak inter-story drift up to $60 \%$, lessen residual deformations in the structure due to excellent re-centering ability, and reduce the deformation demand on the column members at each floor level. Figure 2-14 displays the bracing system with the SMAs, which highlights the SMA connection with either a rigid brace or a buckling restrained brace. 


\section{Beam}

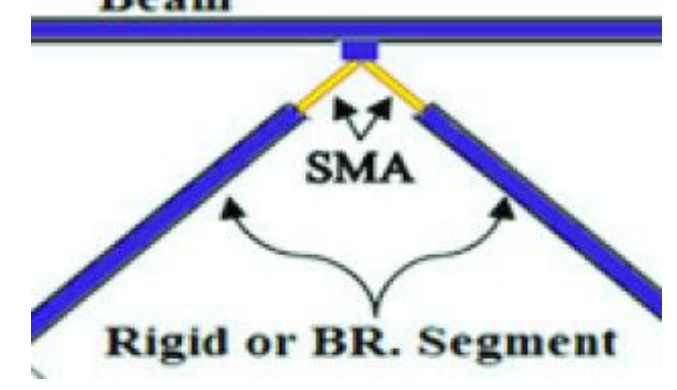

Figure 2-14 Bracing system with SMA connection in two cases (Asgarian and Moradi, 2011)

Miller et al. (2012) developed a high-performance, self-centering, buckling-restrained brace (SC-BRB) as shown in Figure 2-15 and tested the SC-BRB experimentally under the uniaxial quasi-static cyclic loading. The SC-BRB comprised the robust hysteric behavior of two distinct components: a typical BRB component to provide energy dissipation, and superelastic NiTi shape memory alloy (SMA) rods to offer self-centering as well as additional energy dissipation. In this study, the self-centering mechanism was provided by the combination of SMA rods and the BRB, using a configuration of concentric tubes and free-floating end plates that caused the pretensioned SMA rods to elongate when the brace was in either tension or compression. The application of SMA rods contributed large deformations with considerably lower residual deformation, and dissipated energy, as revealed by the flag-shaped hysteretic behavior. The SC-BRB was easily implemented, adopting current design practice, and was employed in place of a steel brace or a BRB following the conventional braced-frame configuration. To validate the innovative brace concept, two half-scale SMA-based SC-BRBs were designed, fabricated, and tested, adopting a standard cyclic loading protocol. The experimental result showed that the use of superelastic NiTi SMAs in the SC-BRB illustrated the promising benefits of the stable hysteretic behavior, including excellent energy dissipation, self-centering capability, and both large maximum and cumulative deformation capacities. The result also demonstrated that the residual brace deformation was controlled by suitably adjusting the ratio of initial SMA pretension force to the strain-hardened $\mathrm{BRB}$ core yield force. 


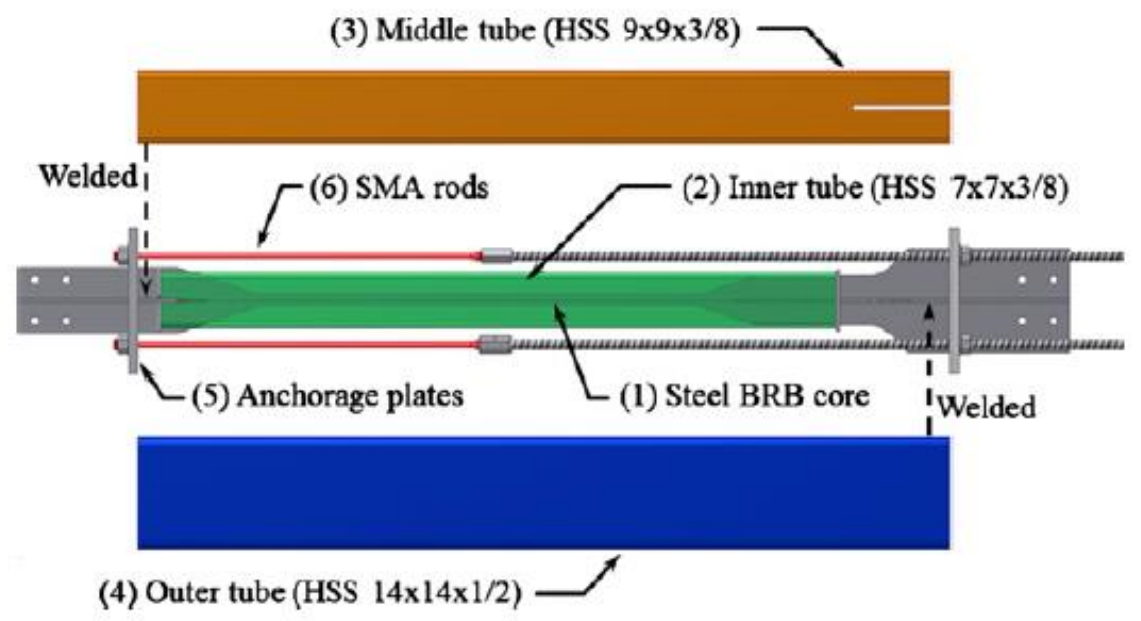

Figure 2-15 SC-BRB components (Miller et al., 2012)

Araki et al. (2016) investigated a steel frame system with a superelastic $C u-A l-M n$ SMA tension brace as a self-centering element in shaking table tests under a series of scaled earthquake ground motions experienced during the 1995 Kobe earthquake in Japan. The large diameter SMA bars, entitled $\mathrm{Cu}-\mathrm{Al}-\mathrm{Mn}$ bars, were recently developed. The results highlighted that the proposed SMA braces were effective in providing self-centering capabilities and in preventing punching. After calibrating the numerical prediction with respect to the response observed from the shaking table tests, the numerical simulations using the calibrated analytical models for the SMA-based tension braces were also performed, under a suite of near-fault ground motions, to access the effectiveness of the SMA braces considering the variability of ground motion records. The results of the validated numerical simulation under near-fault ground motions reinforced the effectiveness of the SMA-based tension brace, indicating an acceptable range of drift angle of less than $0.02 \mathrm{rad}$, and strains in SMA bars of less than 5\%. In this study, a simple stopper, or a deformationrestraining device was also proposed, to ensure extra safety to the frame when experiencing unexpectedly strong ground motions. The test results demonstrated that the installation of stoppers was effective to prevent premature fractures due to imposed loading. Figure 2-16 illustrates the superelastic SMA tension bar with the mechanism of the stopper. 

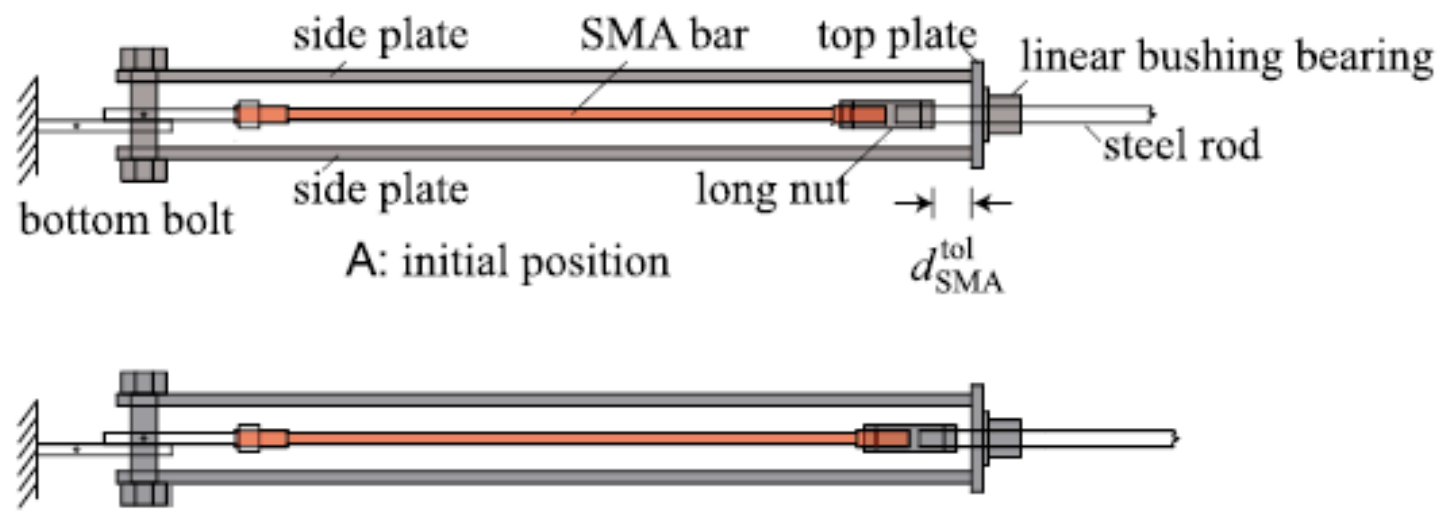

B: before stopper activation

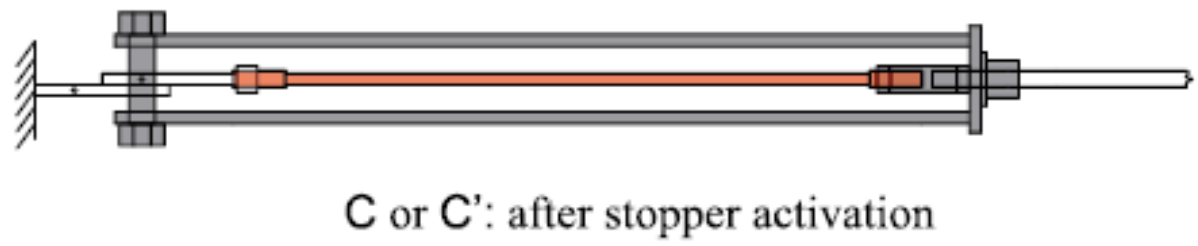

Figure 2-16 Mechanism of the stopper: deformation of the shape memory alloy (SMA) bar (Araki et al., 2016)

\subsubsection{SMA-based Isolation Systems}

Ozbulut and Hurlebaus (2010) explored the seismic performance of an SMA-based, sliding-type, base isolation system under variations of environmental temperatures. As shown in Figure 2-17, the SMA-based isolation system strategically combined a steel-Teflon sliding bearing, which was employed to dissipate energy through frictional behaviors, and NiTi SMA wires, which were used to offer additional energy-dissipating and re-centering capabilities. In order to capture the behavior of superelastic NiTi wires under variations of temperature and loading-rate, a neuro-fuzzy model was used to predict the force of SMA wires. A multi-objective genetic algorithm was employed to determine the length and cross-sectional area of the SMA wires, which were the primary design parameters of the SMA device. A sliding bearing with an SMA device was installed in a multi-span bridge to evaluate the seismic performance of the isolation system under various outside temperatures. The result demonstrated that temperature 
changes indicated a modest influence on the performance of isolated bridge structures. Particularly, the result highlighted that the structural responses of the isolated bridge were affected as follows: a maximum of $13 \%$ variation on the displacement response, and a maximum of $8 \%$ change in acceleration response when the temperature change was $20^{\circ} \mathrm{C}$ from the reference temperature of $20^{\circ} \mathrm{C}$. The result revealed that SMA-based sliding isolators were effective to control the structural response of isolated bridges subjected to earthquake events.

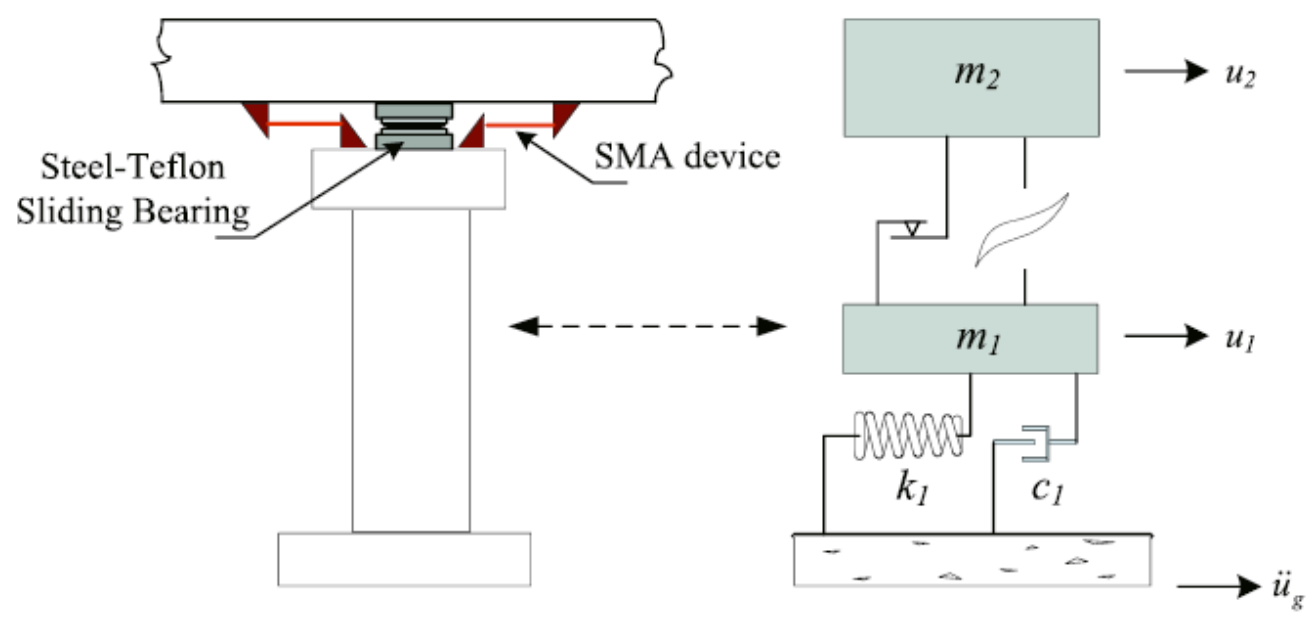

Figure 2-17 A bridge structure modeled with sliding bearings and SMA device (Ozbulut and Hurlebaus, 2010)

Bhuiyan and Alam (2013) conducted the seismic performance assessment of a bridge structure isolated by a high-damping rubber bearing (HDRB) and an SMA-based rubber bearing (SRB), as shown in Figure 2-18 to assess the effectiveness of different isolation systems subjected to moderate to strong ground motion records. For the combined isolation bearing, the SRB consisted of a natural rubber bearing (NRB) wrapped with shape memory alloy (SMA) wires. Two types of combined isolation bearings, SRB-1 and SRB-2, were proposed by using two types of SMA wires, $\mathrm{Cu}-\mathrm{Al}-\mathrm{Be}$ and $\mathrm{Ni}-\mathrm{Ti}$ wires, respectively. The bridge structure isolated with three versions of isolation systems, named as HDRB, SRB-1, and SRB-2, was modeled considering appropriate hysteric behavior for these isolation systems. To simulate the superelastic and damping characteristics of SMA wires, a simplified viscoelastic analytical model was adopted. The 
numerical results demonstrated that SMA-based isolation bearings satisfactorily indicated the superior seismic performance in controlling residual displacement of the bridge deck and the displacement of the bridge pier when subjected to moderate seismic events. However, these results also demonstrated that the SMA-based isolation systems, with $\mathrm{Ni}-\mathrm{Ti}$ and $\mathrm{Cu}-\mathrm{Al}$ - $\mathrm{Be}$, were not able to restrain the residual displacement and pier displacements for strong earthquakes. This study highlighted that the seismic response of the bridge structure was influenced by both the type of isolation bearings and the effect of the modeling of isolation bearings.

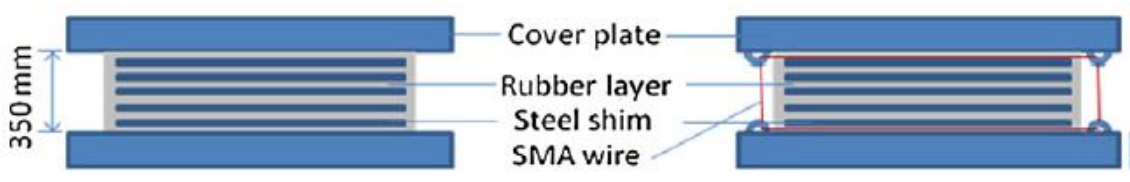

(a)

(b)

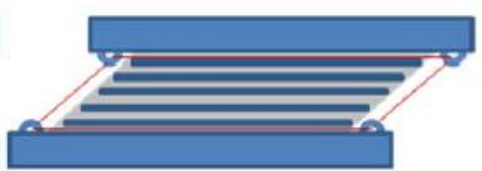

(c)

Figure 2-18 Description of the isolation bearing (a) HDRB; the rubber layers with highdamping properties are vulcanized by steel shims, (b) SRB in un-deformed condition, and (c) SRB in deformed condition (Bhuiyan and Alam, 2013)

Gur et al. (2014) investigated the seismic performance of building structures isolated by the SMA-supplemented rubber bearing (SMARB) as shown in Figure 2-19. The study compared the performance of SMARB over the traditional lead rubber bearing (LRB) under near-fault earthquake records. The numerical study presented the comparative response evaluation of the isolated buildings under a set of historical near-fault, fault-normal components of earthquake records. A parametric study was conducted to identify the optimal characteristic strengths for both the SMARB and LRB systems. Next, the robustness of the seismic performance improvement was also presented, under the varying system characteristics of the superstructure-isolation parameters and the different scenarios of earthquake loadings. The result demonstrated that the seismic performance of the building structure isolated by SMARB was superior over the performance of the conventional LRB under near-fault earthquakes. The result showed that the application of SMARB provided significant improvements in isolation efficiency, indicating considerable reductions of both the peak and residual displacement of the bearings over the application of 
elastomeric bearings, such as LRB. It was also concluded that the SMARB was more effective in providing protection for frequency-sensitive equipment, by suppressing the transfer of highfrequency components of ground motions.

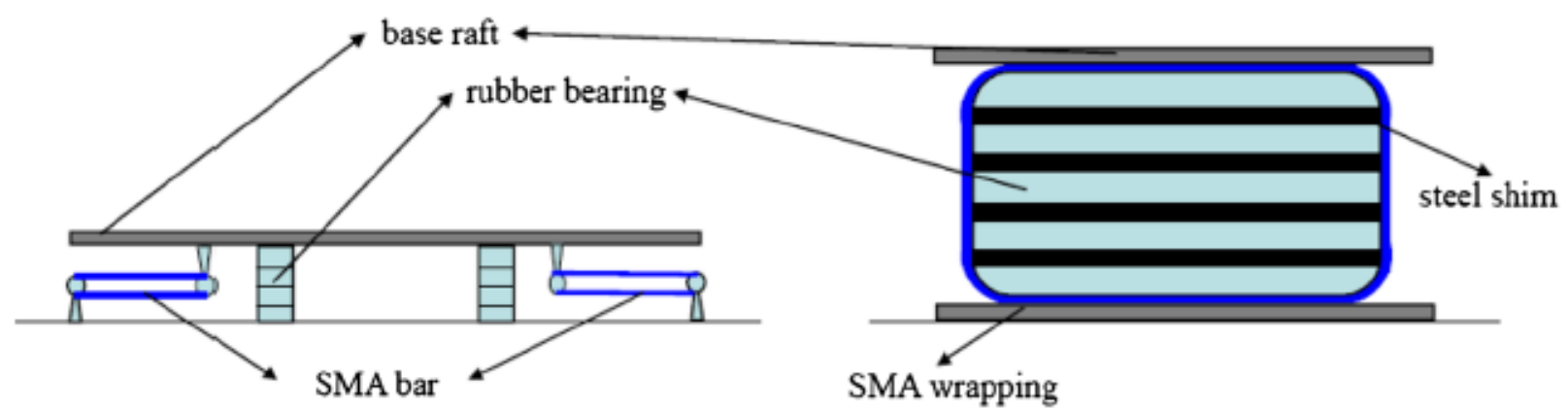

(a)

Figure 2-19 Configurations of the SMARB system: (a) shape-memory alloy (SMA) cable/bar supplementing RB and (b) the rubber bearing with SMA wrap (Gur et al., 2014)

Shinozuka et al. (2015) presented the optimal performance of a building frame isolated by an SMA-based lead rubber bearing (SMA-LRB) under random earthquake events. The SMA-LRB, as shown in Figure 2-20 was proposed by combining the superelastic property of SMA and LRB as the most widely used base isolation system. In this study, a shear building model isolated by the SMA-LRB system was analyzed through nonlinear random vibration analysis to assess the effectiveness of the proposed SMA-based isolation device. Moreover, a bi-objective optimization was formulated to ensure optimal performance of the device by considering two design variables, namely the transformation strength of the SMA and the yield strength of the LRB. The numerical result demonstrated that a base isolation system designed with the set of optimal characteristic strengths of the SMA and LRB was essential to ensure superior performance, by minimizing isolation displacement as well as maximizing isolation efficiency. The results of the parametric study illustrated that the isolation system with optimal design parameters enhanced the robustness of the isolation system under the possible ranges of variations in periods of the system, as well as different earthquake loadings. 


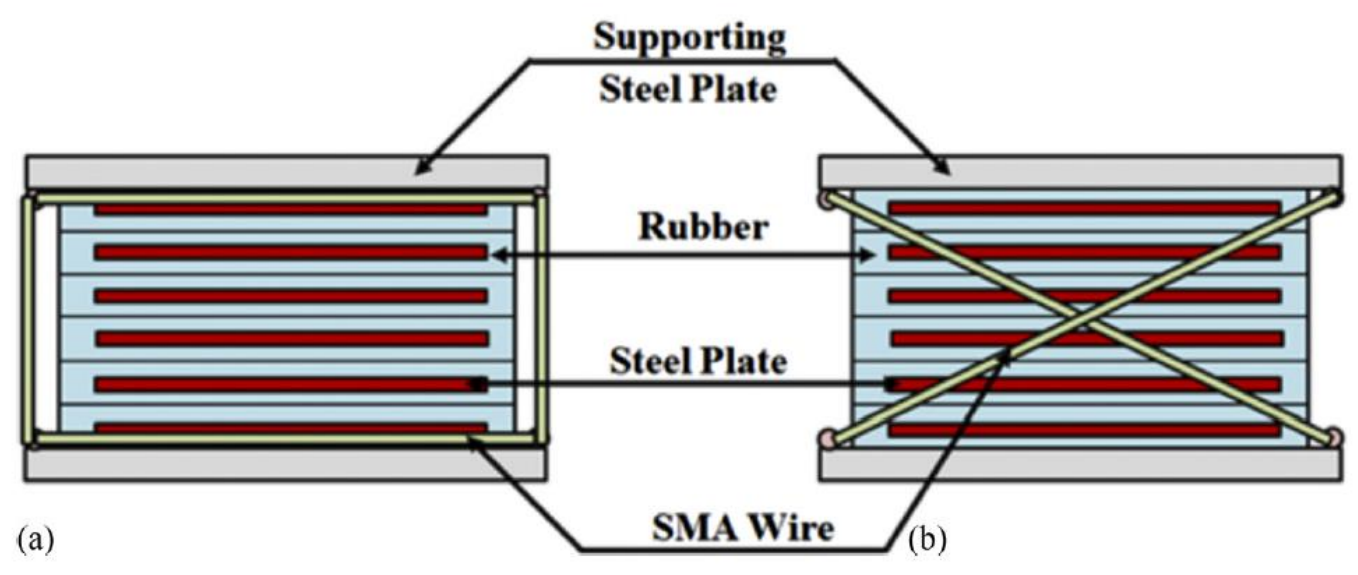

Figure 2-20 Configuration of the isolation system supplemented with SMA (Shinozuka et al., 2015)

Ghodke and Jangid (2017) investigated the influence of high austenite stiffness of shape memory alloy (SMA) used in the elastomeric rubber bearings (ERB), entitled SMA supplement elastomeric rubber bearing (SMARB), to evaluate the structural response of base-isolated buildings subjected to near-fault earthquake excitations. Figure 2-21 shows the configuration of SMARB. The study evaluated the structural response of the building, considering three structural response parameters: top floor acceleration, isolation displacement, and base shear. The effect of the isolation time period, the transformation strength of SMA, and austenite stiffness on the structural response was investigated. The result demonstrated that the SMA-based isolation devices with high austenite stiffness excited the higher modes of the base-isolated structures; thus, higher acceleration associated with higher frequencies were transmitted to superstructures. However, an isolation device with the high austenite stiffness of SMA did not considerably influence the base displacement and base shear of structures. 


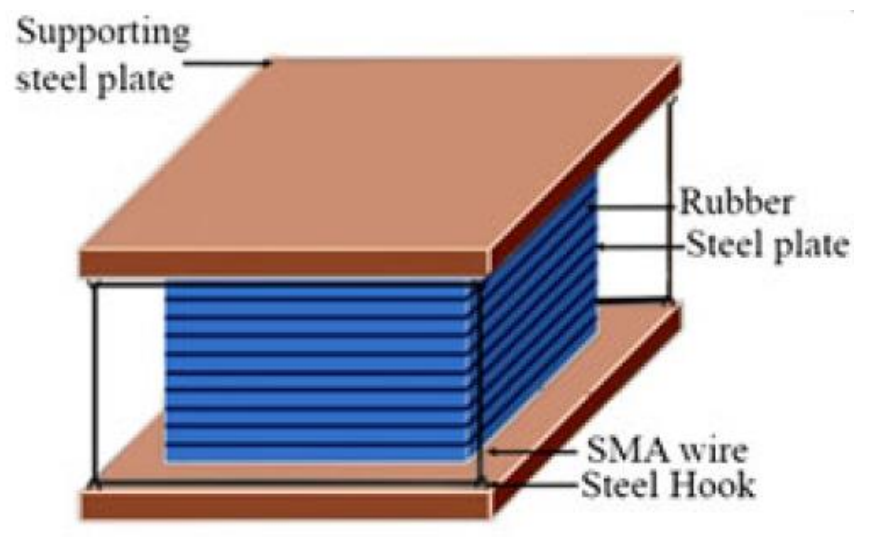

(a)

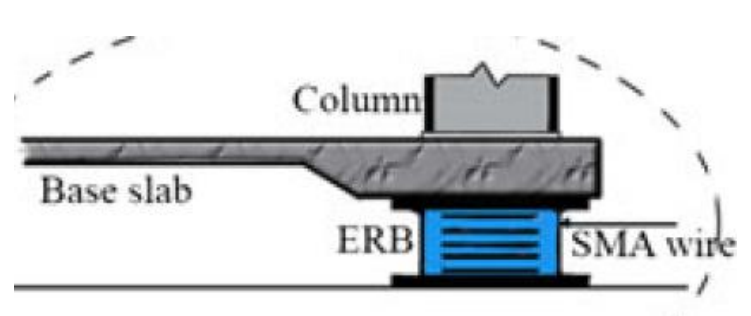

(b)

Figure 2-21 (a) SMA supplement elastomeric rubber bearing (SMARB) and (b) Installation of SMARB at the base isolated building frame (Ghodke and Jangid, 2017)

\subsubsection{SMA-based Dampers}

Zhang and Zhu (2007) investigated the effectiveness of an SMA-based reusable hysteric damper (RHD) in the seismic response control of civil engineering structures subjected to strong earthquake events. The RHD, as shown in Figure 2-22 was developed to provide distinctive features, such as the tunable hysteric behavior and the ability to withstand several design-based earthquakes by utilizing superelastic Nitinol stranded wire for energy dissipation. In the design of the RHD, design parameters, such as the inclination angle of the SMA wires, pretension levels, and friction coefficients of the damper, were adjusted to achieve the suitable hysteretic behavior of the damper while using the device for passive structural control. The hysteric behavior of the RHD device was validated through the experimental test results, and an analytical model of the RHD was developed to predict its response. A parametric study was conducted to examine the influence of design parameters on the damper's energy-dissipating performance. The numerical simulation of a three-story steel building with and without RHDs was conducted to evaluate the

effectiveness of the RHD as a passive structural control of structure systems. The results 
demonstrated that the RHD device was effective in reducing the structural response of steel frame structures excited by strong earthquake events.

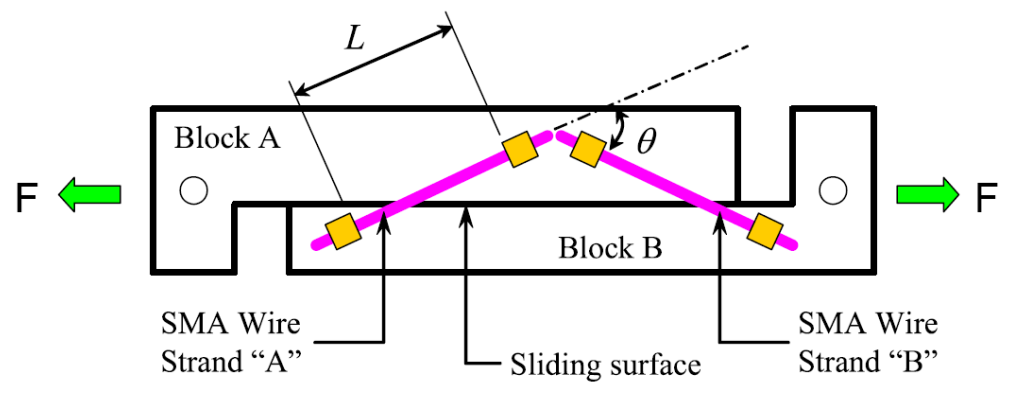

(a)

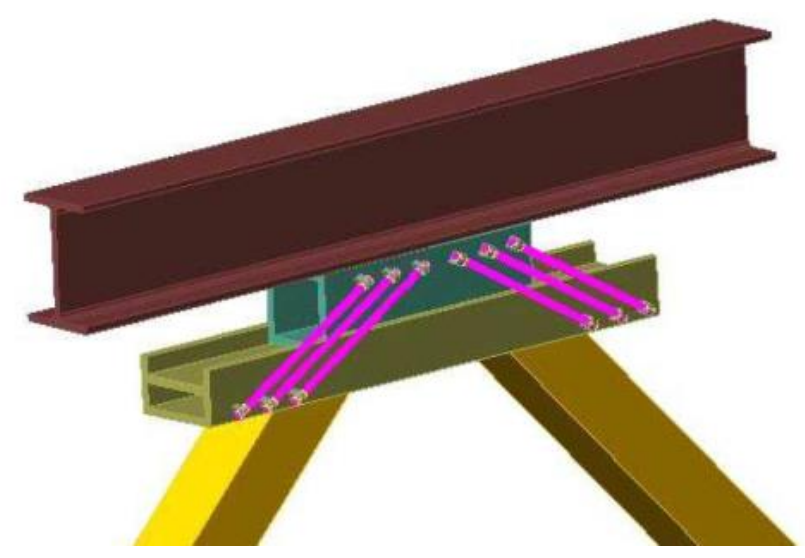

(b)

Figure 2-22 Schematics of the (a) SMA-based reusable hysteretic damper, (b) an RHD in a steel framed building (Zhang and Zhu, 2007)

Zhu and Zhang (2008) also investigated the self-centering capability of a special SMAbased bracing element named as self-centering friction damping brace (SFDB), as shown in Figure 2-23. The SFDB was developed by leveraging the re-centering capability of superelastic Nitinol wires and the enhanced energy dissipation capability of a friction-based mechanism. The dynamic analyses of three- and six-story steel concentrically braced frames (CBFS) were conducted for a comparative evaluation of SFDB frames and buckling restrained braced (BRB) frames subjected to two suites of historical ground motions for Los Angeles. The self-centering behavior of the 
SFDB was obtained by suitably selecting the ratio between the yield strength of the Nitinol SMA and the friction force developed in the device. The result demonstrated that SFDB frames were capable of providing a comparable seismic response to that of BRB frames, in terms of peak interstory drift, while reducing the residual drift considerably. Furthermore, the results highlighted the enhanced seismic performance of the SFDB due to the potential benefit of the frictional damping in the device. The comparative study concluded that the SFDB revealed promising characteristics, in withstanding several design-based earthquakes with no need of replacement, due to the full strain recovery feature of superelastic SMA wires.

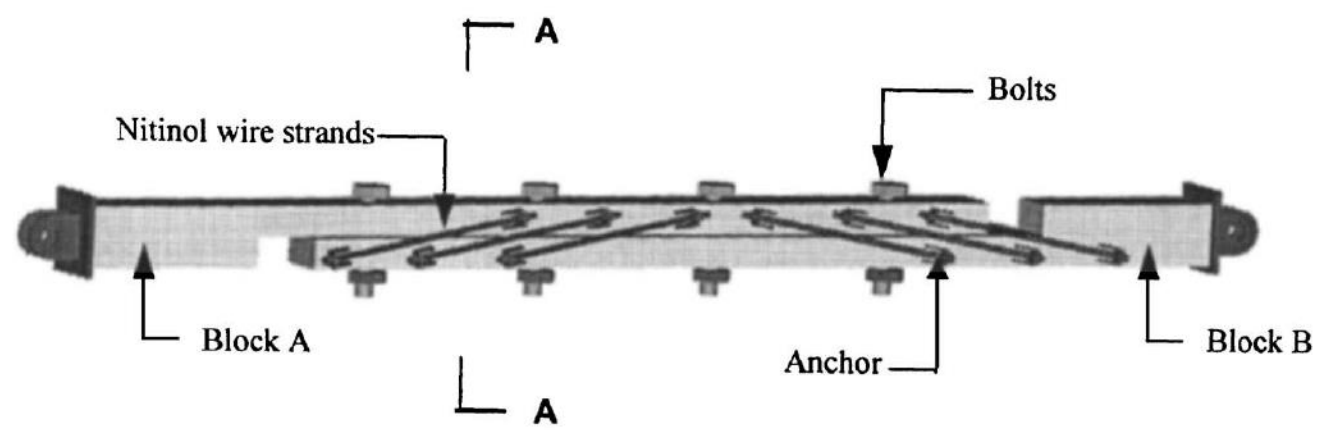

Figure 2-23 Schematic of mechanical configuration of SFDB (Zhu and Zhang, 2008)

Ma and Cho (2008) proposed an innovative SMA-based damper with full re-centering capability. As shown in Figure 2-24, the new damper was composed of the pretensioned superelastic SMA wire to contribute energy dissipation, and two precompressed springs to offer re-centering ability. In the damper device, the pretensioned SMA wires and roller system provided a high-energy dissipation capacity, while these springs supplied an expected restoring force to the damper, providing full re-centering capability by altering the precompression applied to the springs. Numerical studies were performed, using the Vrinson's constitutive model for the SMA material, to validate the expected behavior of the damper. The analytical results showed that the meter-long SMA-based damper demonstrated full re-centering capability with the equivalent 
damping ratio of 0.12 , which was obtained when the SMA wire loops revealed a high energy dissipation capacity at $3 \%$ pre-strain and $2.8 \%$ strain amplitude, to a $30 \mathrm{~mm}$ stroke.

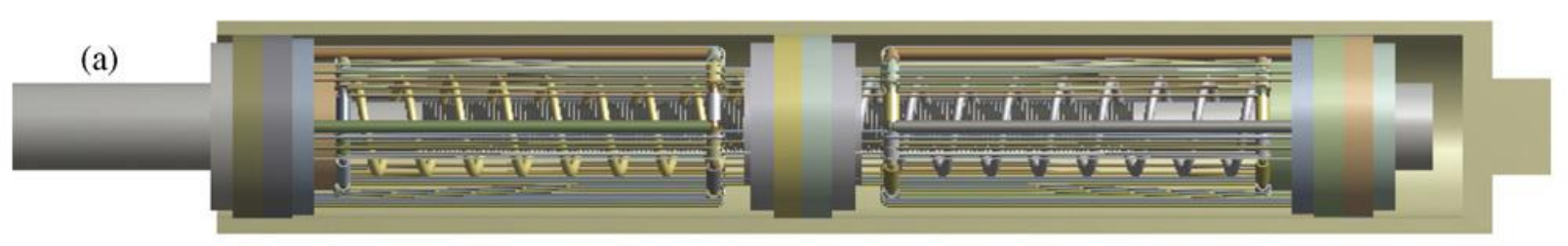

Figure 2-24 Schematic diagram of the SMA damper (Ma and Cho, 2008)

Qian et al. (2013) evaluated the effectiveness of a re-centering shape memory alloy damper (RSMAD) to mitigate the seismic response of structures. The RSMAD was developed utilizing the superelastic Nitinol wires (SMA) as the kernel energy-dissipating component as shown in Figure 2-25. In this study, improved constitutive equations for the superelastic Nitinol wires were proposed, based on the Graesser and Cozzarelli model and validated by the cyclic tensilecompression tests on the dampers, with different prestrains under various loading frequencies and displacements. The results from the experimental test validated the hysteric behavior generated, based on the improved constitutive model, reflecting the martensitic hardening characteristics of SMAs under large amplitudes, with superior performance both in the re-centering and energydissipating features under various conditions. In the numerical simulation, a ten-story frame with SMA dampers in five different configurations, and also without the dampers, was investigated, considering different earthquake ground motions, to access the effectiveness of RSMADs for structural seismic protection. The simulation results revealed that the SMA-based dampers were capable of significantly reducing structural vibrations, indicating their effectiveness as an energydissipating device. 


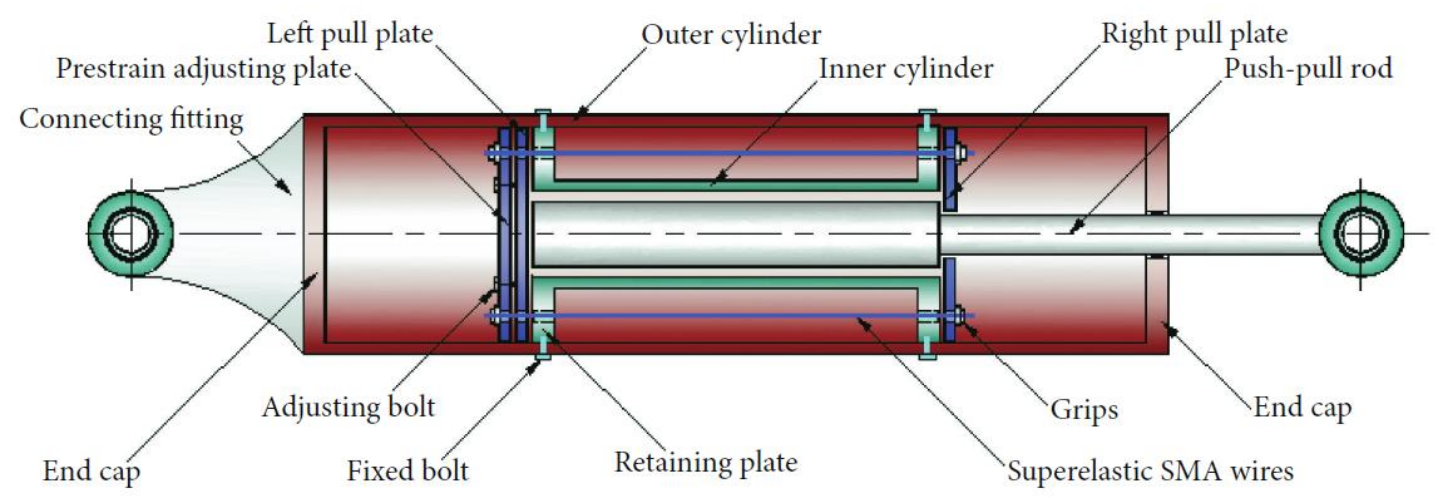

Figure 2-25 Proposed RSMAD device (Qian et al., 2013)

Yang et al. (2010) proposed an SMA-based hybrid device, which provided the dual characteristics of energy-absorbing and re-centering capabilities, to mitigate structural damage during seismic events. The hybrid damper, shown in Figure 2-26(a) and (b), consisted of three main components: (i) a set of re-centering SMA material; (ii) two energy-absorbing struts- and (iii) two high-strength steel tubes to facilitate the movement of the device. The length of the SMA wires was designed such that their strain reached within the target strain of $6 \%$, which indicated full re-centering capability and avoided the SMA stiffening phase even in the occurrence of large deformations. Furthermore, the energy-absorbing struts were designed to be seismically compact and stocky to avoid the buckling issue in compression loading. The numerical study of a threestory SAC building was conducted to explore the behavior and performance of the building installed with the hybrid devices in two different configurations: horizontally between a beam and braces of a frame, or utilized simply as a diagonal brace, as shown in Figure 2-26(c) and (d). The results from the numerical study concluded that the SMA-based hybrid dampers contributed performances comparable to the BRBF system in energy dissipation capacity and peak inter-story drift, while also indicating superior re-centering capabilities. 


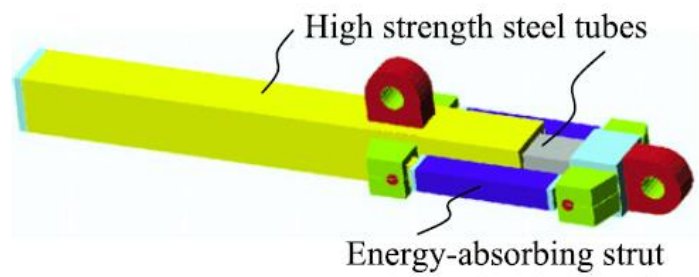

(a)

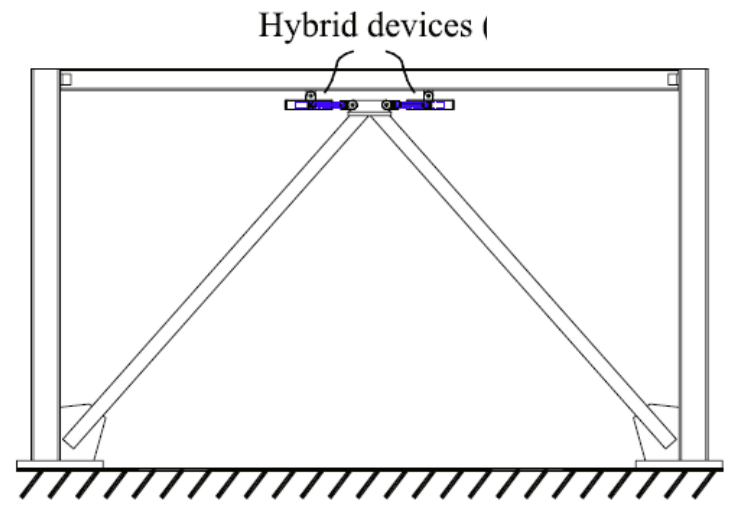

(c)

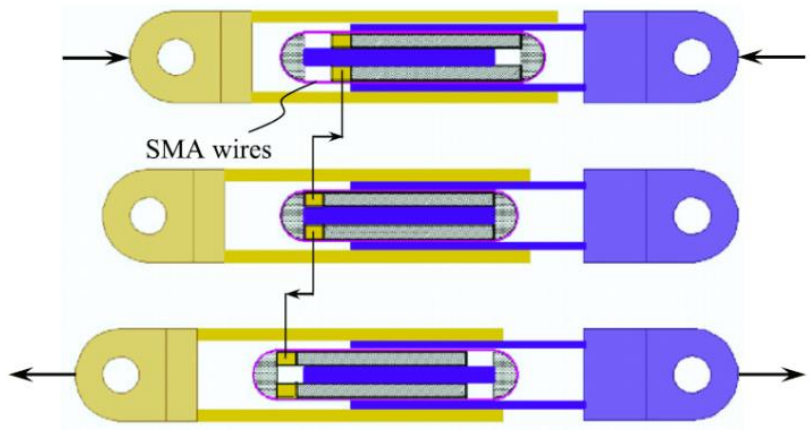

(b)

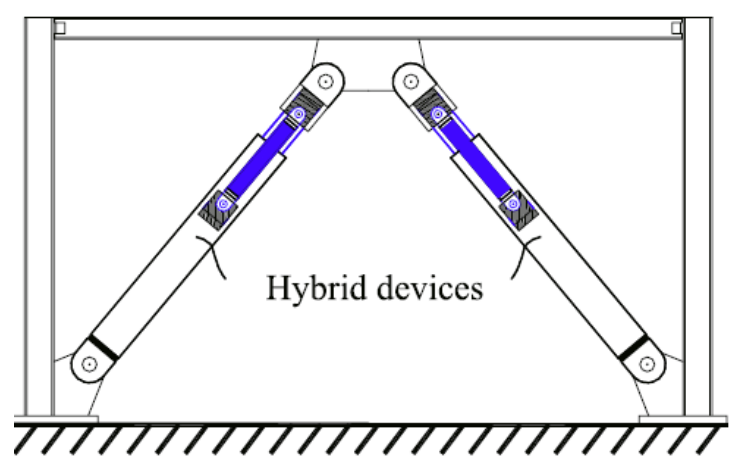

(d)

Figure 2-26 (a) SMA wires inside a hybrid device with clevis pins for chevron bracing; (b) SMA wires inside a hybrid device with clevis pins for diagonal bracing; (c) arrangement of hybrid devices in chevron bracing; (d) arrangement of hybrid devices in diagonal bracing (Yang et al., 2010)

Qian et al. (2016) investigated the effectiveness of a superelastic shape memory alloy friction damper (SSMAFD) in controlling the seismic response of structures, through experimental testing of a three-story steel frame building. The SSMAFD was proposed by combining the unique features of pretensioned superelastic SMA wires and friction components, as shown in Figure 2-27. The SMA wires and the integrated friction devices were primarily employed to contribute recentering ability and energy dissipation ability, respectively. In shake table tests, a quarter-scale building structure was experimentally tested to assess the seismic performance of the device under historical earthquake records. The structural models with or without SSMAFD were analyzed 
under various earthquake loadings to evaluate their dynamic behaviors in terms of story displacements, inter-story drift, and story accelerations. The shake table test results revealed that the frame building installed with SSMAFD was capable of controlling the dynamic response of the buildings subjected to strong earthquake events. Furthermore, the SSMAFD demonstrated superior energy dissipation capability with remarkable re-centering ability.

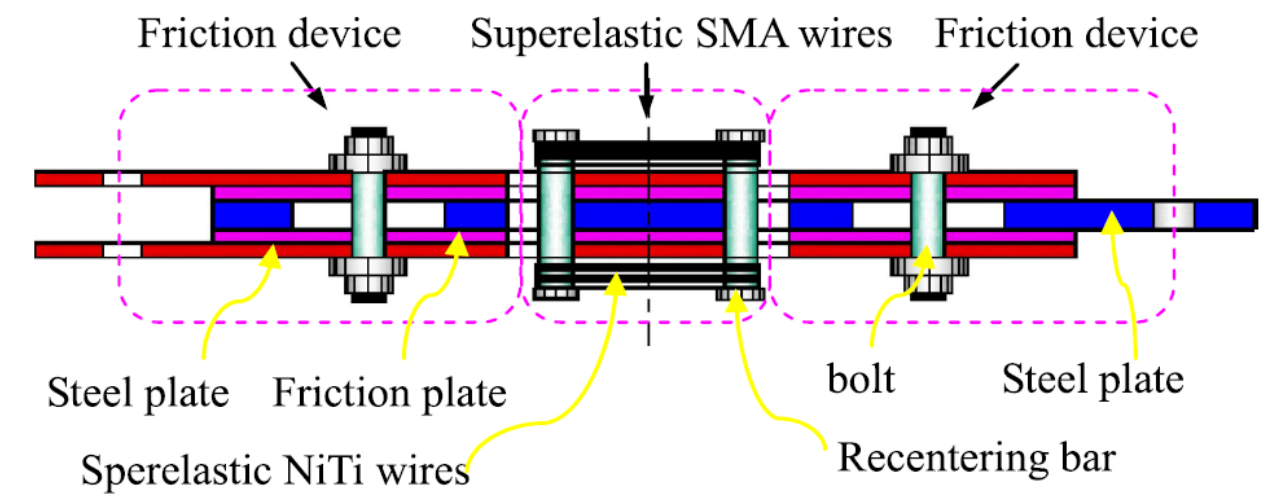

Figure 2-27 Scheme diagram of SSMAFD (Qian et al., 2016)

Bhaowmick and Mishra (2016) numerically explored the seismic performance of a new $\mathrm{Fe}$-based SMA ( $\mathrm{Fe}$ - $\mathrm{Ni}$-Co-Al-Ta-B, entitled FNCATB) damper, as an alternative to Nitinol or $\mathrm{Cu}$ Al-Be SMAs. Figure 2-28 illustrates the schematic diagram of the proposed damper. The performance of the superelastic FNCATB damper installed in a single-bay and single-story structural frame was compared with a frame with Nitinol and $\mathrm{Cu}$ - $\mathrm{Al}$-Be-based devices. The optimal performance of the damper was achieved by selecting the parameters of the damper to maximize its equivalent damping. The force-deformation behavior of the damper was validated by fitting the available experimental test data with a cyclic stress-strain model proposed by the well-known Auricchio model. The numerical results illustrated that structural systems exhibited a superior performance with the proposed FNCATB damper, in terms of reducing story drift. The study concluded that the new FNCATB damper with a $F e$-based SMA could be a cost-effective alternative because of its ferrous constituent. 


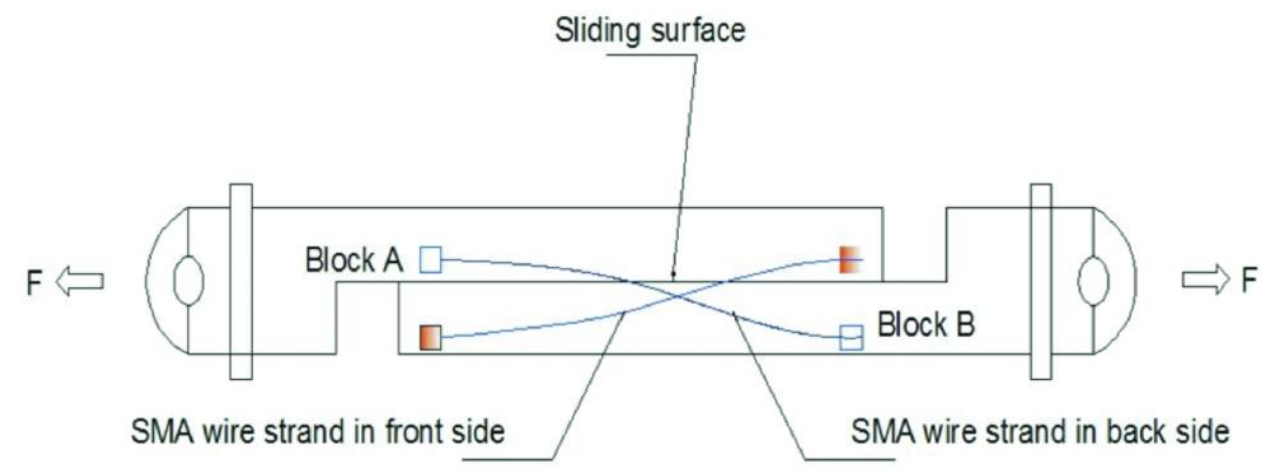

Figure 2-28 Schematic configuration of the superelastic damper (Bhaowmick and Mishra, 2016) 



\section{SUPERELASTIC VISCOUS DAMPER}

\subsection{Introduction}

This section presents the description of a new hybrid passive control device named as a superelastic viscous damper. The proposed superelastic viscous damper (SVD) relies on shape memory alloy cables for re-centering capability and employs a viscoelastic damper that consists of two layers of a high damped blended butyl elastomer compound to augment its energy dissipation capacity. In what follows, the experimental studies on the individual components of the hybrid damper, namely SMA cables and a butyl elastomer, are described first. Then, the design and the behavior of the proposed superelastic viscous damper are introduced.

\subsection{Description of SVD}

\subsubsection{Experimental Characterization of SMA Cables}

Shape memory alloy cables have been recently developed as an alternative and new structural element. They leverage the superior mechanical characteristics of small diameter SMAs into large-size structural tension elements. Besides, they have considerable cost advantages over same size monolithic SMA bars (Reedlunn et al., 2013). In this study, SMA cables are considered for the development of a hybrid seismic device. The SMA cable is made of Nickel Titanium (NiTi) and obtained from Fort Wayne Metals, Research Products Corp. The SMA cable, which is produced in a helix configuration, is composed of 7 strands and each strand has 7 wires as shown in Figure 3-1. Each wire has a diameter of $0.885 \mathrm{~mm}$ providing outer cable diameter of $8 \mathrm{~mm}$ and total cross sectional area of $30.14 \mathrm{~mm}^{2}$. 


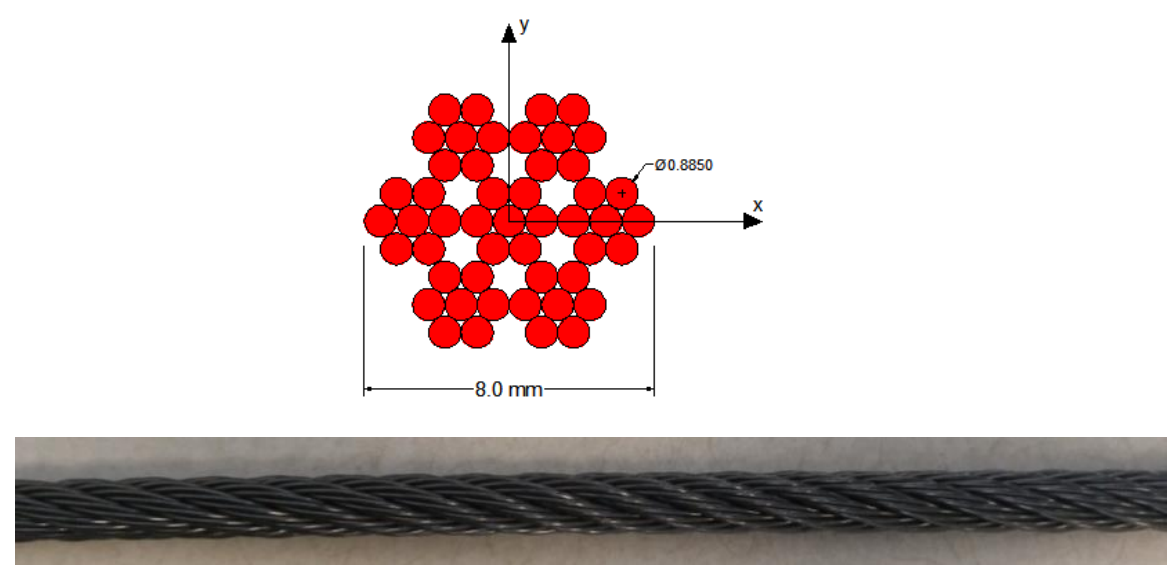

Figure 3-1. Shape memory cable: cross-sectional and longitudinal views.

The uniaxial tensile tests are conducted at various loading rates and strain amplitudes to characterize the superelastic properties of the SMA cable and to study the rate-dependent mechanical response of the SMA cable under dynamic loads (Ozbulut et al., 2015). The test samples are obtained by cutting the cable into pieces with a length of $150 \mathrm{~mm}$. Before conducting formal tests, a training test procedure that consists of 20 load cycles at strain amplitude of 5\% at $0.01 \mathrm{~Hz}$ is applied. The displacement and force data are recorded using MTS data acquisition system. The strains are also measured using a laser extensometer.

Figure 3-2(a) shows stress-strain curves from experimental tests at measured strain amplitudes varying from $1.5 \%$ to $7.7 \%$. It can be observed that the material exhibits well-known flag-shaped cycles, which is a common behavior of SMAs. The SMA cable recovers almost all of its deformations upon unloading when it is loaded up to strain amplitude of $6.5 \%$. On the other hand, recorded residual strains at strain amplitudes of $7.2 \%$ and $7.7 \%$ are only about $0.2 \%$. It can be also seen that the strength of the cable decreased at high strain amplitudes possibly due to the failure of individual wires at the gripping region.

The tensile loads are also applied to the cable in a displacement controlled test to obtain $6 \%$ target strain at loading frequencies of $0.1,0.5,1.0$, and $2.0 \mathrm{~Hz}$. Figure 3-2(b) shows the hysteresis loop at each loading frequency. It can be observed that both the forward and reverse transformation stresses increase with the increasing test frequency. However, the increase in the 
reverse transformation stress level is more pronounced. Therefore, the area under the hysteresis loop, which signifies the energy dissipation, slightly decreases for the higher loading rates.

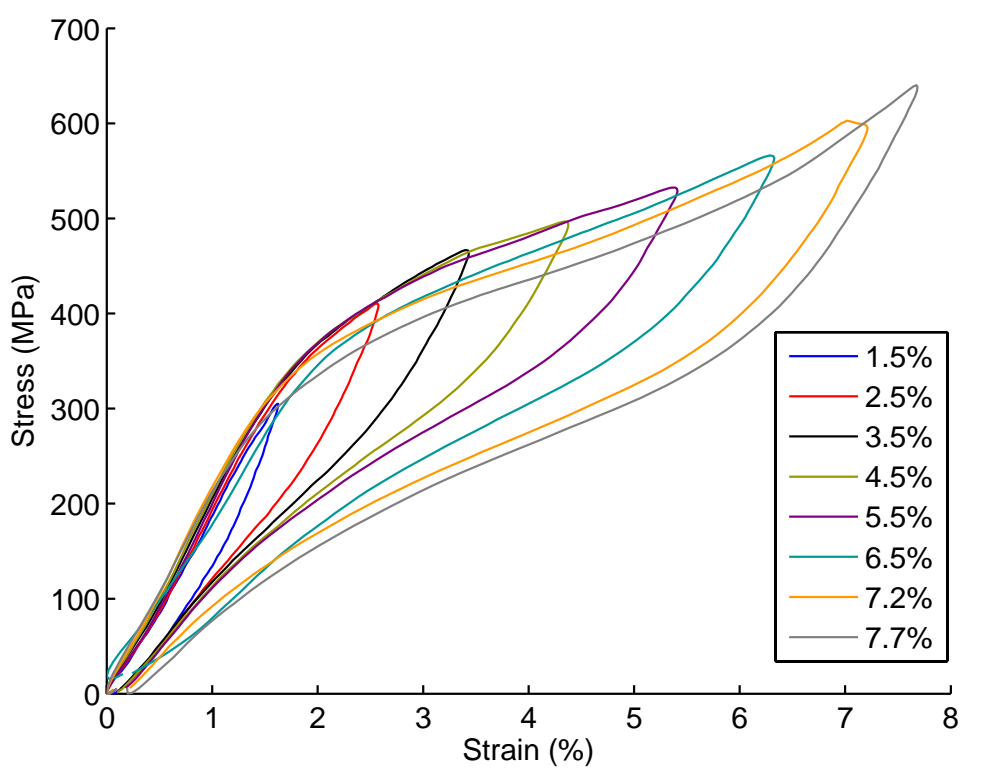

(a)

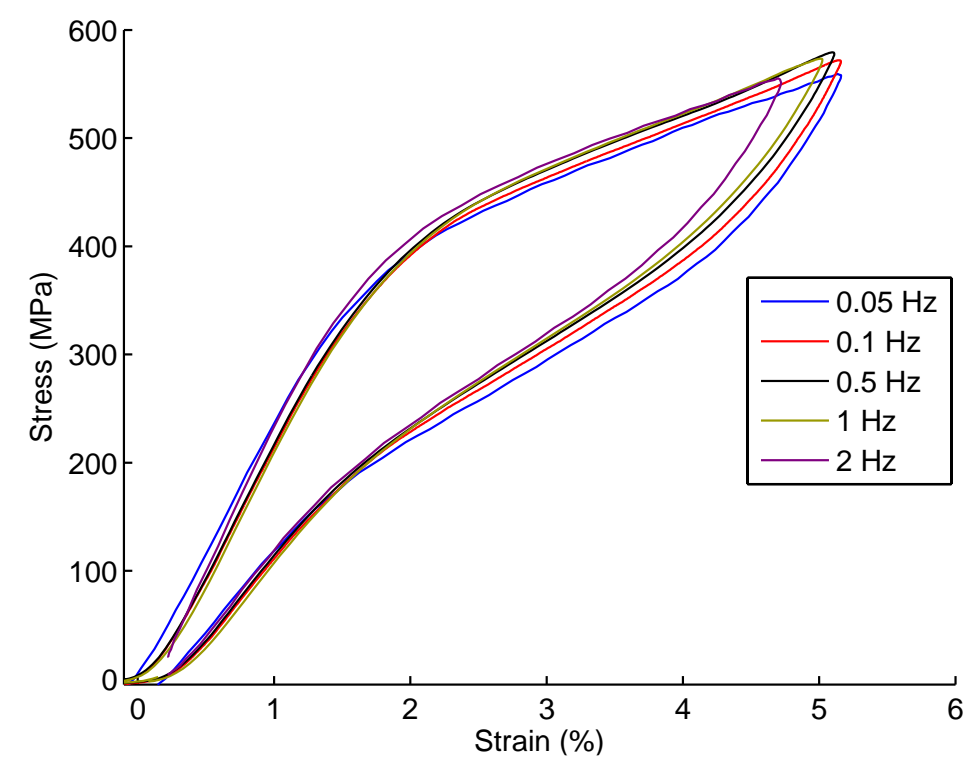

(b)

Figure 3-2. Stress-strain curves of SMA cable under different (a) strain amplitudes and (b) loading frequencies. 


\subsubsection{Experimental Characterization of High Damped Butyl Elastomer}

The high damped (HD) butyl series is compounded specifically to produce high damping at moderate to low stiffness. These elastomers are currently in use in various VE dampers and in a new base isolation system used to isolate storage racks from seismic events (Sause and Ricles, 2012; Michael et al., 2012; Sweeney and Michael, 2006). For these isolation devices, the HD butyl has resulted in an increase in damping by more than a factor of two over traditional elastomers. In this study, the HD butyl compounds are considered for use in an SMA-based hybrid damper.

Butyl rubber is a synthetic rubber produced by polymerization of about $98 \%$ isobutylene with about $2 \%$ of isoprene. Butyl rubber is also known as polyisobutylene or PIB. It has excellent impermeability, inherently high damping and its long polymer chains give it excellent flex properties. The first major application of butyl was tire inner tubes because of its excellent impermeability to air. Butyl is also used extensively in vibration isolators due to its high damping. Other favorable properties include a low glass transition temperature, low modulus, low compression set and excellent resistance to aging and to weathering from atmospheric exposure.

The HD butyl compounds are highly loaded which means that the \% rubber hydrocarbon $(r h c)$ is lower than traditional industrial butyls. Since there is less elastomer in it, the damping tends to go up because carbon black and oil, the typical materials used to lower the rhc, generally have higher damping than the rubber alone. In addition, these compounds utilize a grade of carbon black that builds hardness slowly so in order to get an equivalent hardness to traditional butyls, more black is needed which tends to drive up damping. Also these compounds use a higher viscosity plasticizer, which tends to drive up damping even more.

Natural Rubber (NR) is a multi-purpose elastomer and the elastomer of choice for most conventional seismic base isolators (where supplemental damping such as a lead core is added). NR rubber offers high tensile strength, high elongation and resilience, good fatigue, low damping, as well as low cost. It should be noted that the NR polymer is intrinsically resilient and its formula does not contain ingredients that contribute heavily to damping.

In order to obtain material properties and illustrate the differences of a typical NR compound, typical industrial butyl and HD butyl, static and dynamic shear tests are conducted at Gannon University. The NR and industrial butyl compounds are 50 durometer Shore A, and HD 
butyl is 45 durometer Shore A. All testing performed is to ASTM D5992, which utilizes the Fast Fourier Transform (FFT) method to determine important elastomer properties such as elastic shear modulus $\left(G^{\prime}\right)$, dynamic shear modulus $\left(G^{\prime \prime}\right)$, loss factor $\left(\eta=G^{\prime \prime \prime} G^{\prime}\right)$ and dynamic elastic stiffness $\left(K_{e}\right)$. The specimen geometry is a double shear with two identical rubber elements symmetrically disposed on opposite sides of a central rigid member. This specimen geometry is specified in ASTM D5992 and carefully designed to yield a length/wall thickness ratio of $8(=1.600 / .200)$ to achieve a state of pure shear.

The static shear load deflection curves shown in Figure 3-3(a) illustrate the difference in stiffness between the three compounds. The curve for NR and butyl shows an increase in stiffness at larger strains due to strain crystallization. For HD butyl, static modulus changes most at lower strains and softens by a factor of 2 as the strain increases from $10 \%$ to $200 \%$. However, for strain values between $100 \%$ and $200 \%$, the modulus is nearly constant (curve is nearly linear). Since the actual application strain will be in this range for a seismic application, it can be assumed that the modulus and therefore stiffness will be somewhat constant for the actual operating strain range. The stress-strain hysteresis loops recorded for $\pm 100 \%$ strain and $1 \mathrm{~Hz}$ under shear for the three materials are shown in Figure 3-3(b). In addition, Figure 3-4 compares various mechanical properties of three compounds. It can be seen that the NR and industrial butyl have higher static and dynamic shear modulus than the HD butyl. The HD butyl is significantly softer than the NR and industrial butyl yet has slightly more damping. The combination of low stiffness and heavily damped is atypical for an elastomer and a direct result of compounding described above. For the hybrid damper proposed in this study, the viscoelastic component is expected to provide damping without adding significant stiffness, i.e. the HD butyl is an ideal elastomer for this application. 


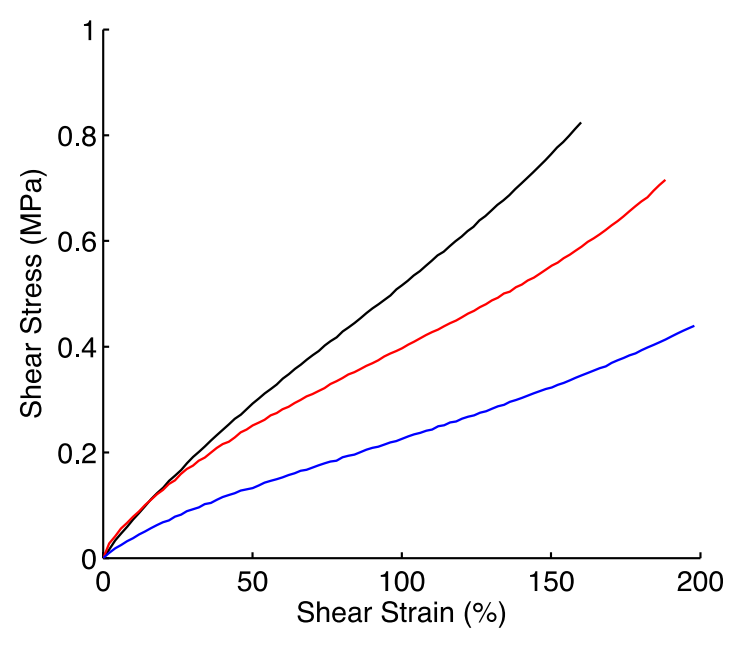

(a)

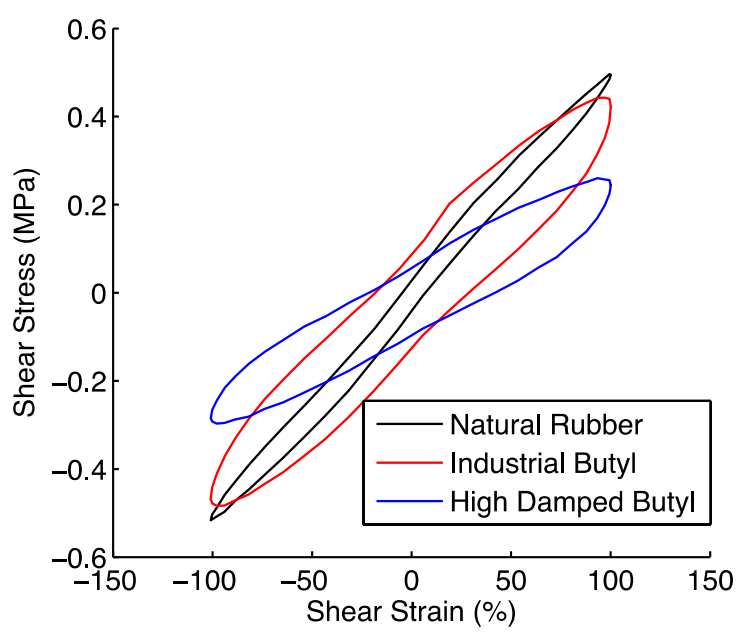

(b)

Figure 3-3. (a) Static shear stress versus shear strain, (b) Hysteresis loops at a frequency of 1 $\mathrm{Hz}$ and $100 \%$ strain for three compounds

In order to investigate the effects of strain amplitude and loading frequency on the mechanical properties of the HD butyl, shear tests are conducted at up to four specific and common frequencies $(0.1,0.5,1.0$, and $2.0 \mathrm{~Hz})$ and at up to three specific and common strain (displacement) amplitudes $(50 \%, 100 \%$, and 200\%). In actual seismic application, the elastomer strain is expected to see between $100 \%$ and $200 \%$ strain and a frequency of $0.5 \mathrm{~Hz}$ to $2 \mathrm{~Hz}$. So the test range is adequate to determine mount behavior in actual use. All tests are conducted at room temperature. All samples are pre-flexed (conditioned) twice to eliminate Mullin's effect. Mullin's effect is a softening that occurs during the first several cycles of deformation due to breaking of weak bonds. After the first few cycles, the material stabilizes with little change in subsequent cycles. Figure 3-5(a) shows shear strain-stress curves at a constant frequency of $1 \mathrm{~Hz}$ and cyclic strains of $\pm 50 \%$, $\pm 100 \%$ and $\pm 200 \%$ while the other subplots of Figure 3-5 illustrate the hysteresis loops under various loading frequencies for the HD butyl at various strain amplitudes. Moreover, Figure 3-6 plots the variation of dynamic shear modulus, loss factor and elastic stiffness with loading frequency at different shear strains. 

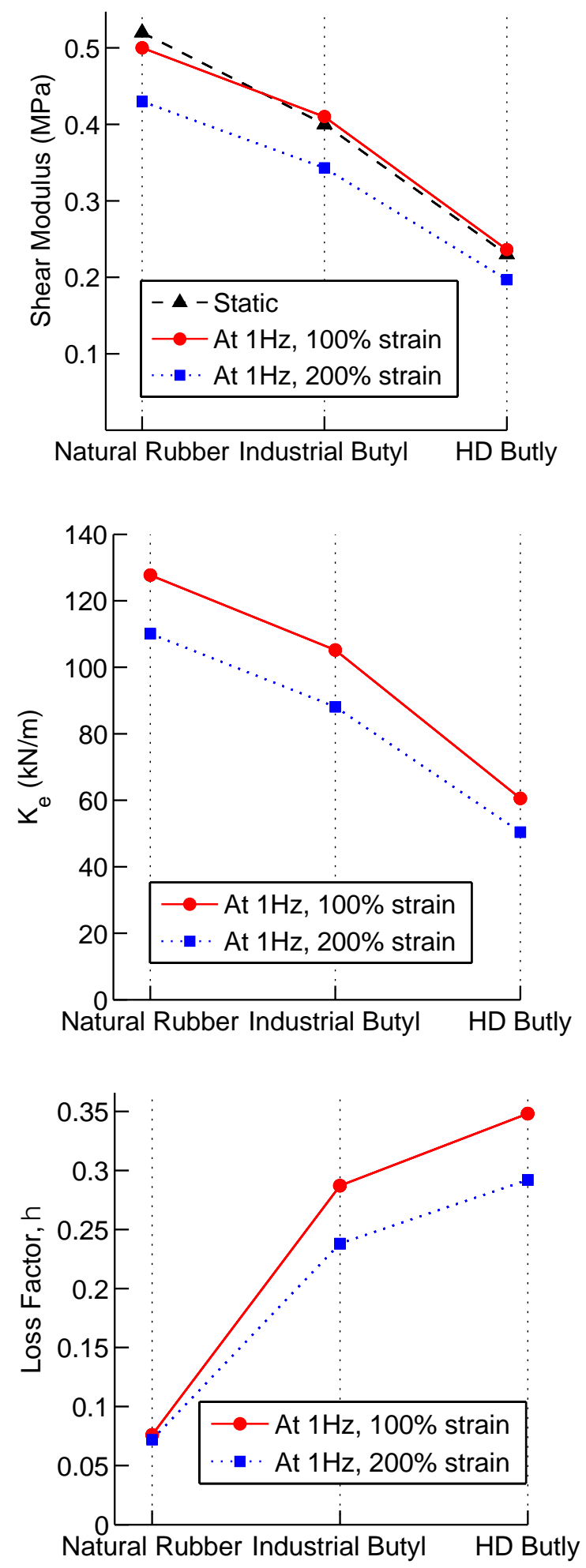

Figure 3-4. Shear modulus, elastic stiffness and loss factor for three compounds 


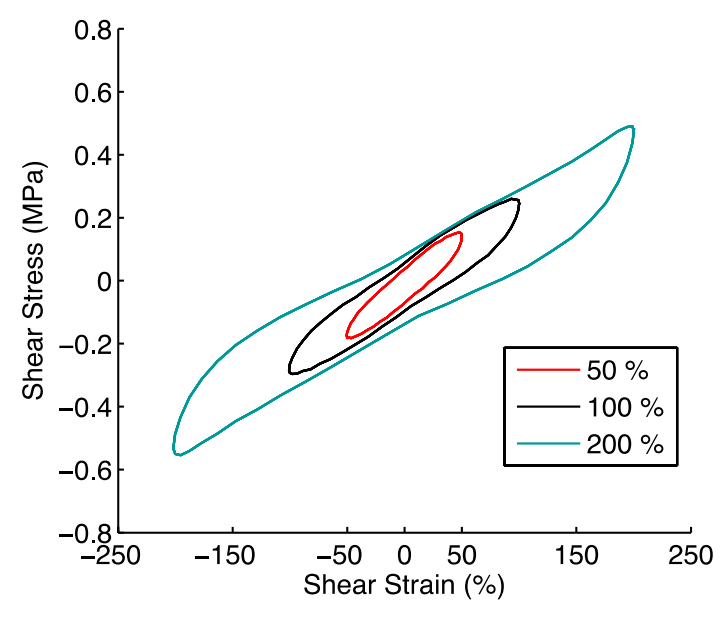

(a)

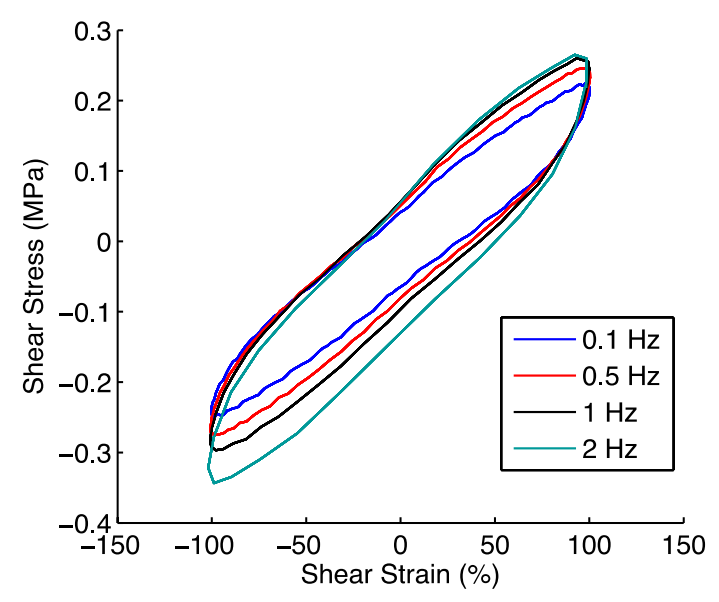

(c)

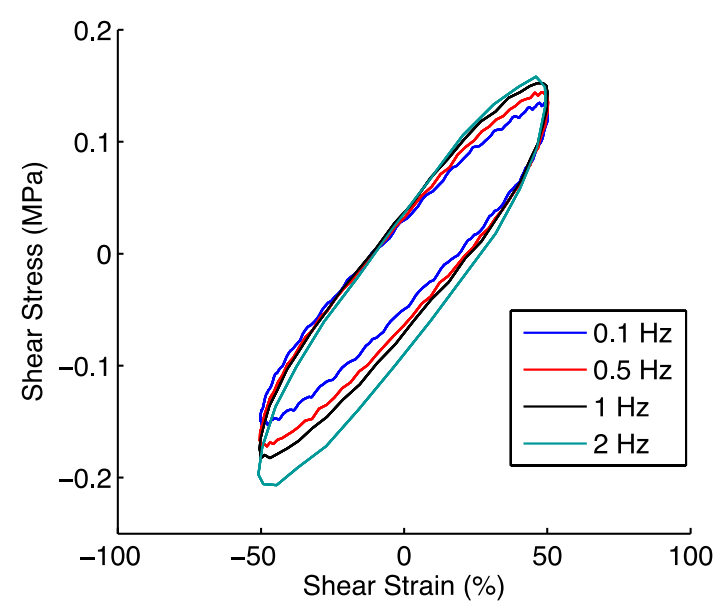

(b)

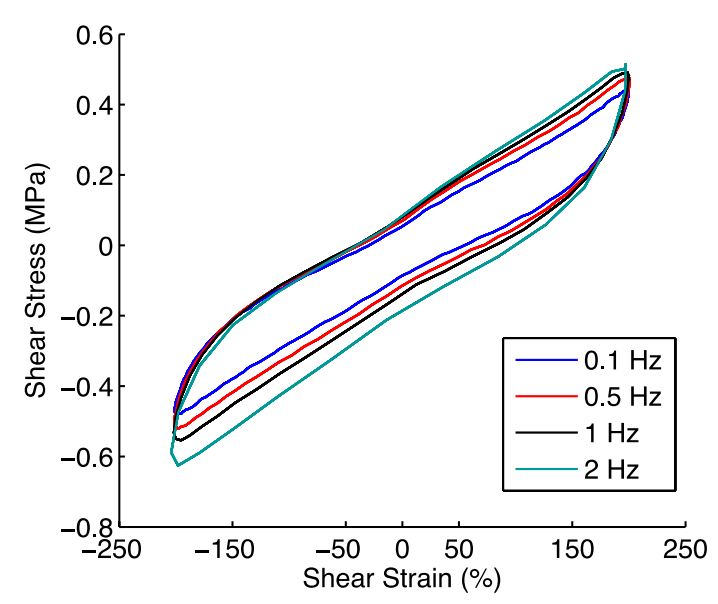

(d)

Figure 3-5. Hysteresis loops for HD butyl (a) at various strain amplitudes at $1 \mathrm{~Hz}$, and at various frequencies at (b) $50 \%$ strain, (c) $100 \%$ strain, and (d) $200 \%$ strain.

The results reveal that the dynamic shear modulus and elastic stiffness increases with increasing loading frequency. The properties seem to be more frequency sensitive at smaller dynamic strains. In other words, the modulus and stiffness vary less (only 5\% increase) at $\pm 200 \%$ than they do (about $16 \%$ increase) at $\pm 50 \%$ dynamic strain across the frequency range of $0.1-2$ Hz. Furthermore, dynamic shear modulus and elastic stiffness are affected by strain amplitude 
more as compared to loading frequency. Both decrease about $37 \%$ when the strain amplitude is varied from $50 \%$ to $200 \%$.

The loss factor is also impacted by strain amplitude and loading rate. It varies from 0.37 at $50 \%$ to 0.28 at $200 \%$ cyclic strain at $1 \mathrm{~Hz}$, which corresponds to a $20 \%$ decrease. On the other hand, the loss factor increases with increasing loading rate. In particular, there is a $29 \%$ and $28 \%$ increase in loss factor at $50 \%$ and $100 \%$ shear strains, respectively when the loading frequency is changed from $0.1 \mathrm{~Hz}$ to $2 \mathrm{~Hz}$, while the same increase is $19 \%$ at $200 \%$ strain.
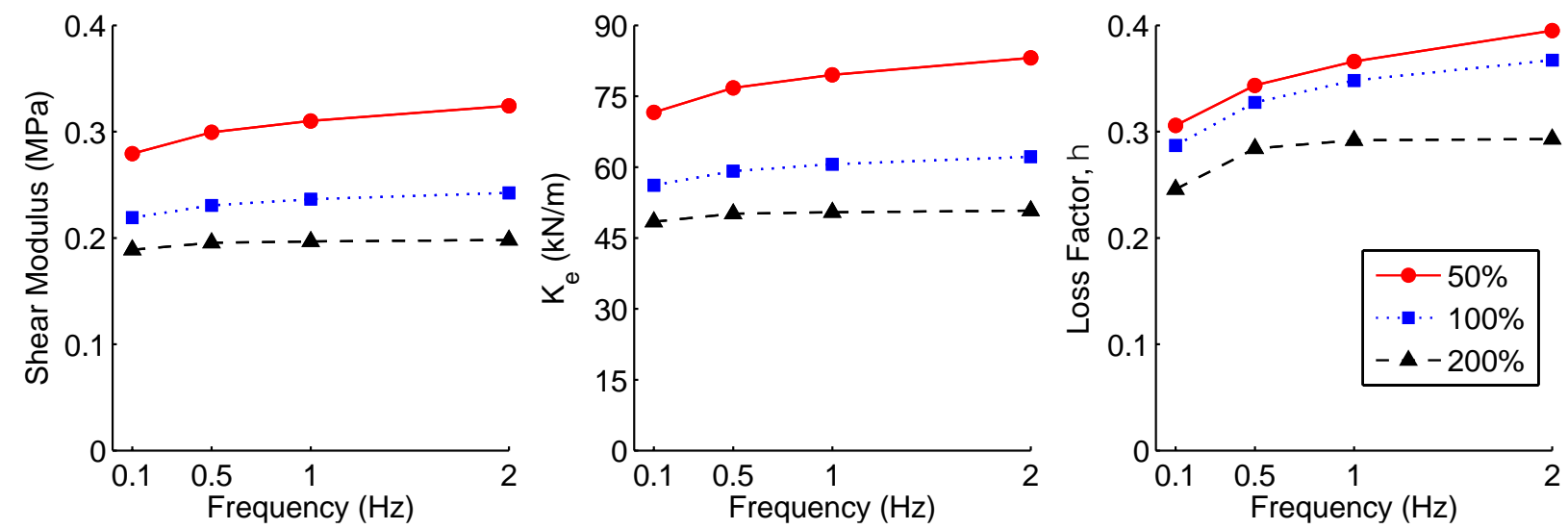

Figure 3-6. Variation of shear modulus, elastic stiffness and loss factor for HD butyl with loading frequency

\subsubsection{Superelastic Viscous Damper}

The proposed Superelastic Viscous Damper (SVD) combines re-centering capabilities of shape memory alloys with the energy dissipation ability of viscoelastic devices. The 3D renderings of the SVD and schematic diagrams of the device in the undeformed and deformed positions are given in Figure 3-7(a) and (b). The module comprises two high damped elastomer compounds, sandwiched between and bonded to three identical steel plates and installed SMA cables. Each SMA cable forms a continuous loop; wrapping the loops around the outer two plates improves compactness and efficiency. Whether the device itself moves left or right, the configuration ensures that the SMA elements will remain under tension. Along the top and bottom of the device, the wires are threaded through guides, which ensure that the wires remain parallel to the direction 
of shear deformation in the elastomer layers. As the device moves through its design displacements, the SMA cables apply a re-centering force to the center plate through a second set of wire guides. Composed of $3 \mathrm{C}$-channels, 2 angle brackets, and 4 crescent-shaped steel sections (one for each strand), each guide distributes the design loads over a longer length of wire (the arclength of each crescent, compared to the thickness of the center plate) in order to eliminate problematic stress concentrations.

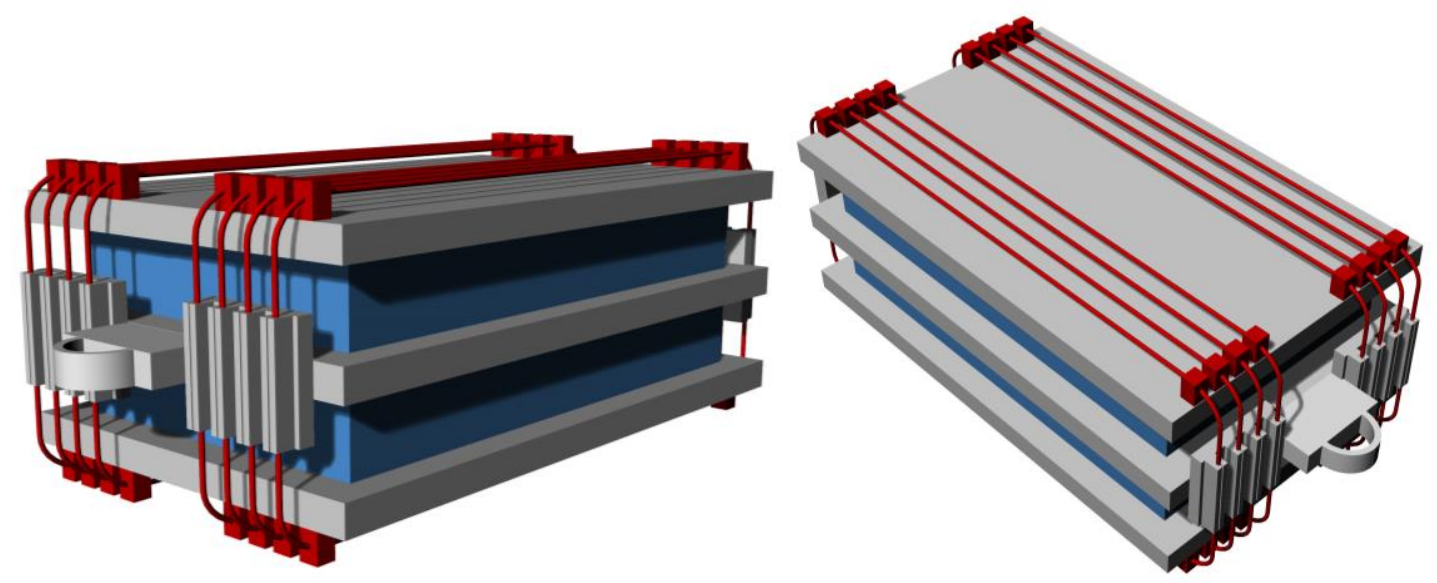

(a)

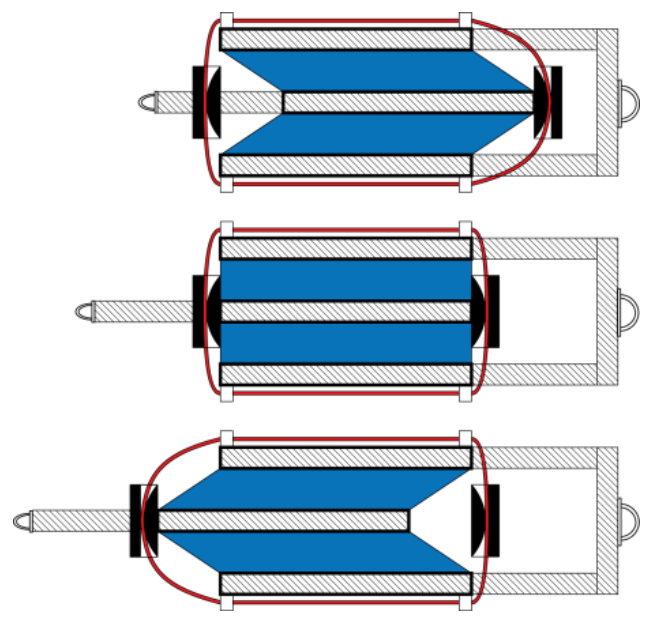

(b)

Figure 3-7. (a) 3D rendering of SVD, (b) a schematic diagram of SVD at its undeformed and deformed positions 


\subsection{Design of SVD}

Here, the SVD device is designed for a displacement capacity of $100 \mathrm{~mm}$. Assuming $200 \%$ shear strain at the design displacement for the elastomer compound, the thickness of each butly elastomer can be obtained as:

$$
t=-=\frac{100(\mathrm{~mm})}{200(\%)}=50 \mathrm{~mm}
$$

When the SVD moves through its design displacement $(\delta)$, the elongation of the SMA cables can approximately be calculated as:

$$
\delta_{S M A}=2\left(\sqrt{(b / 2)^{2}+\delta^{2}}-b / 2\right)
$$

where $b$ is the height of the SMA elements. Note that the effect of crescent-shape guides on the elongation of SMA elements is neglected in the above equation. The total length of each SMA cable, which is given as $\mathrm{L}_{\mathrm{SMA}}=2(\mathrm{c}+\mathrm{b})$ in Figure 3-8, is determined such that the maximum strain on the SMA cables at the design displacement of the damper remains in the superelastic strain range of the SMAs. Choosing $\mathrm{b}=185 \mathrm{~mm}$ and $\mathrm{c}=432 \mathrm{~mm}$ yields $\mathrm{L}_{S M A}=1235 \mathrm{~mm}$. Then, the strain on the SMA cable calculated as $\varepsilon_{S M A}=\delta_{S M A} / L_{S M A}$ is about $7.1 \%$. The total cross-sectional area of the SMA cables and the shear area of the viscoelastic component are designed such that the maximum force capacity and equivalent viscous damping ratio of a single damper will be about $300 \mathrm{kN}$ and $10 \%$, respectively. To achieve these design objectives, the cross-sectional area of the SMA cable is set to be $241 \mathrm{~mm}^{2}$. The selected cross-sectional area corresponds to 8 SMA cables with a diameter of $8 \mathrm{~mm}$ for each device. The required area for one layer of elastomer compound is $0.165 \mathrm{~m}^{2}$. Hence, the dimension of each elastomer compound is set to be $406 \mathrm{x} 406 \mathrm{x} 50 \mathrm{~mm}$. 


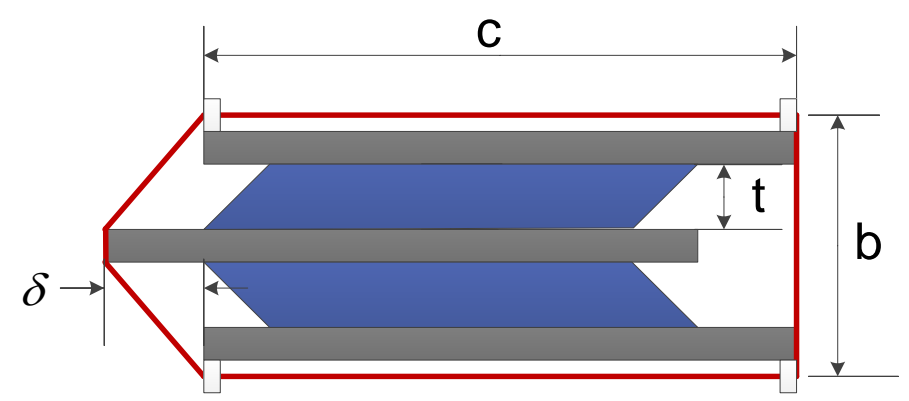

(c)

Figure 3-8. Design parameters for SVD

\subsection{Hysteretic Behavior of SVD}

Figure 3-9 shows the typical force-deformation curves of the SVD and its subcomponents at two different displacement amplitudes. It can be seen that the energy is dissipated by viscoelastic component at relatively small displacements while the SMA behaves almost in a linear-elastic manner and provide additional stiffness. At higher displacement amplitude, both the SMA cables and viscoelastic contribute to the energy dissipation, while the SMA elements provide additional re-centering force as a result of their superelastic behavior. Furthermore, the softening nonlinear behavior of SMAs at large displacements limits the base shear when the deformations become large. 


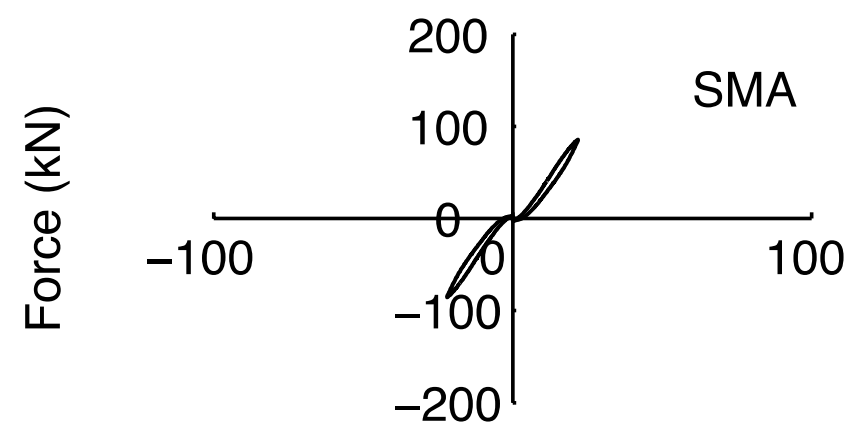

Displacement $(\mathrm{mm})$

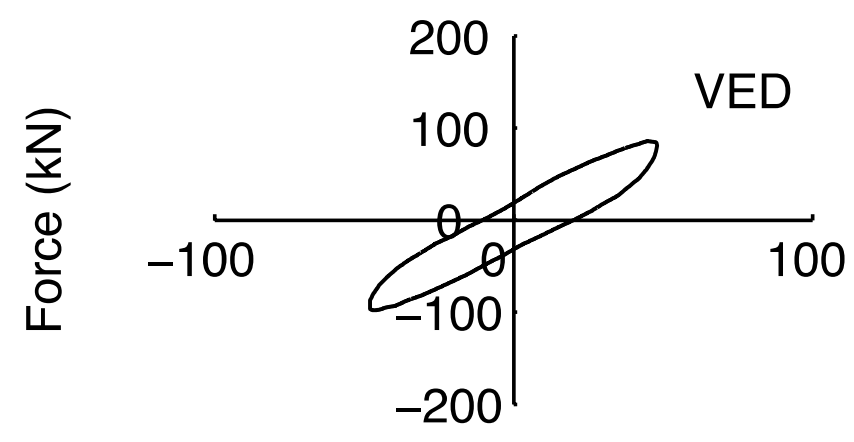

Displacement (mm)

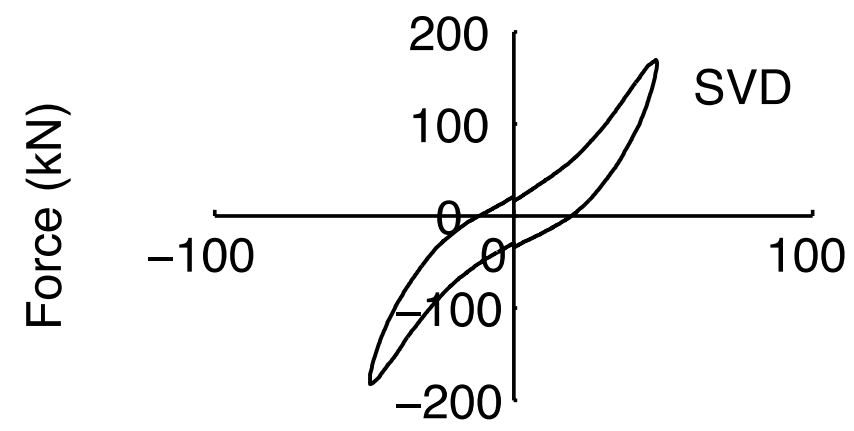

Displacement $(\mathrm{mm})$

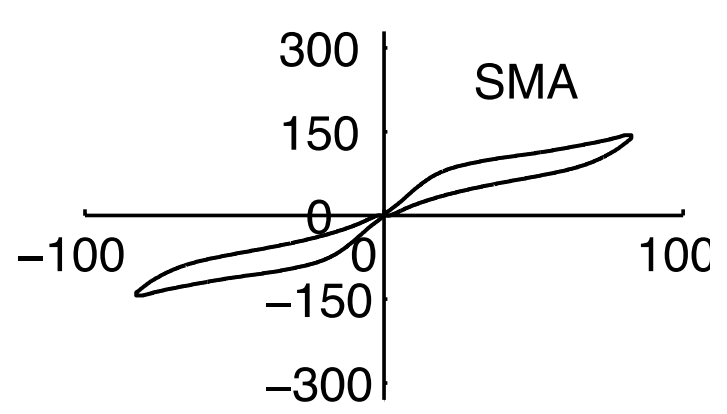

Displacement (mm)

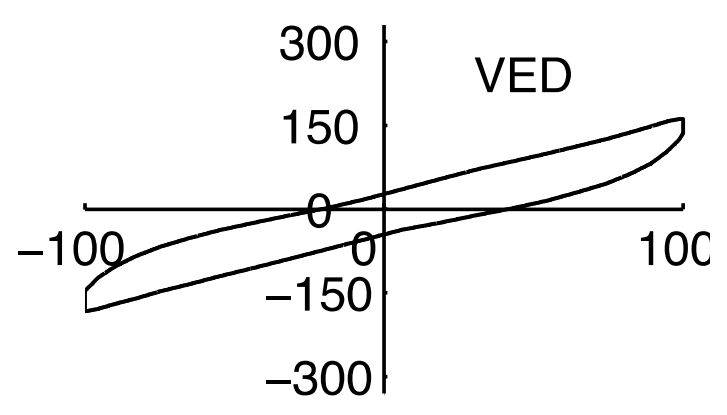

Displacement $(\mathrm{mm})$

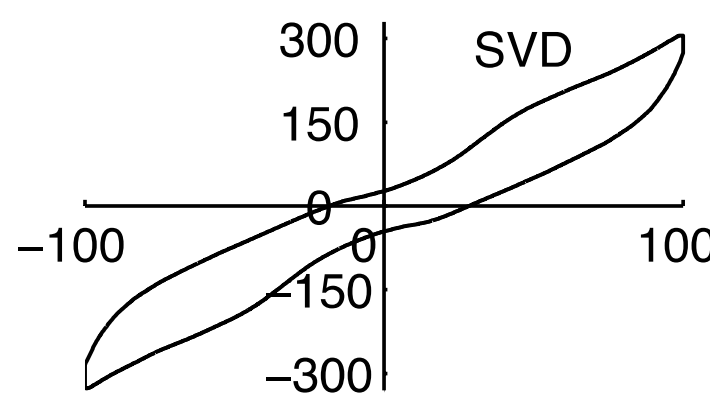

Displacement (mm)

Figure 3-9. Force-deformation curves of the SVD and its sub-components (SMA and VED) at two different displacement amplitudes 


\subsection{Closure}

A hybrid passive damper, which leverages the inherent re-centering capability of SMA cables and energy dissipation ability of an elastomer compound for superior seismic performance, is proposed. A heavily damped butyl compound, which provides high damping at low stiffness, is considered for the viscoelastic component of the hybrid damper, named as superelastic viscous damper. Experimental tests on the subcomponents of the hybrid damper, i.e. HD butyl compound and SMA cables, are conducted to characterize their mechanical response. The configuration of the SVD is described and its design parameters are discussed. The hysteresis loops of the SVD based on experimental testing of its subcomponents is provided at two different displacement levels. 


\section{SEISMIC RETROFITTING OF STEEL FRAME BUILDINGS WITH SVDS}

\subsection{Introduction}

The objective of this section is to explore the effectiveness of the proposed superelastic viscous damper (SVD) in the seismic retrofitting of steel frame buildings. First, analytical models for a six-story frame building illustrated in the FEMA P-751 (FEMA, 2012) are developed in OpenSees, a finite element framework for the nonlinear and dynamic analysis of structural systems. The building was analyzed in FEMA P-751 through a nonlinear response history analyses and excessive story drifts were identified, especially at lower floor levels. A damping system was suggested to upgrade the performance of structure. In this study, the superelastic viscous dampers are installed to enhance the seismic response of the selected building. Next, following the seismic design requirements for structures with damping systems described in Chapter 18 of ASCE/SEI 710, a nonlinear response-history procedure is adopted to design the steel frame with SVDs. A total of 7 ground motions are selected from PEER NGA database (PEER, 2014) and scaled according to ASCE/SEI 7-10 for the use in nonlinear analyses. In order to assess the efficiency of the SVD system, the performance of the six-story frame with installed SVDs is compared with response quantities of the bare frame.

\subsection{Model of Frame with Installed SVDs}

\subsubsection{Building Description}

In order to assess the performance of the proposed damper in mitigating seismic response of structures, a six-story steel building illustrated in the FEMA P-751, NEHRP Recommended Seismic Provisions: Design Examples (FEMA P-751, 2009) is selected for numerical analyses. The building is designed as an office building located in Seattle, Washington on class $\mathrm{C}$ soil. The special steel moment-resisting frames on the perimeter of the building provide the lateral load resistance for the structure. The building consists of five bays at $8.53 \mathrm{~m}$ (28 feet) in the northsouth $(\mathrm{N}-\mathrm{S})$ direction and six bays at $9.14 \mathrm{~m}$ (30 feet) in the east-west $(\mathrm{E}-\mathrm{W})$ direction. A plan and elevation of the building in the $\mathrm{N}-\mathrm{S}$ direction are shown in Figure 4-1. All the analyses in this 
study are for lateral loads acting in the $\mathrm{N}-\mathrm{S}$ direction. The story height is $3.81 \mathrm{~m}$ (12 feet-6 in.) at each floor except the first floor, which has a height of $4.57 \mathrm{~m}$ (15 feet).

One of the perimeter steel special moment frames that serve as the seismic-force-resisting system of the structure is analyzed. All the columns in the $\mathrm{N}-\mathrm{S}$ direction bend about their strong axis and the girders are attached with fully welded moment-resisting connections. The building is designed with Reduced Beam Section (RBS) connection details in accordance with design standards, ASCE/SEI 7-05 (ASCE, 2005) and ANSI/AISC 341-05 (AISC, 2005). The building is assigned to Seismic Design Category (SDC) D. The design spectral acceleration parameters are $\mathrm{S}_{D S}=0.912 \mathrm{~g}$ and $\mathrm{S}_{D 1}=0.459 \mathrm{~g}$, while the maximum considered spectral acceleration values are $\mathrm{S}_{\mathrm{I}}=1.368 \mathrm{~g}$ and $\mathrm{S}_{M 1}=0.689 \mathrm{~g}$. The seismic mass of the second level is $1.248 \times 10^{6} \mathrm{~kg}$, the mass of the third level through sixth level is $1.242 \times 10^{6} \mathrm{~kg}$ and the mass of the roof level is $1.237 \times 10^{6}$ $\mathrm{kg}$. The fundamental period of the structure is 1.96 seconds. The building was analyzed in FEMA P-751 through a nonlinear response history analysis and excessive story drifts were identified, especially at lower floor levels. A damping system was suggested to upgrade the performance of structure. In this study, the superelastic viscous dampers are used to enhance the seismic response of the selected building. 

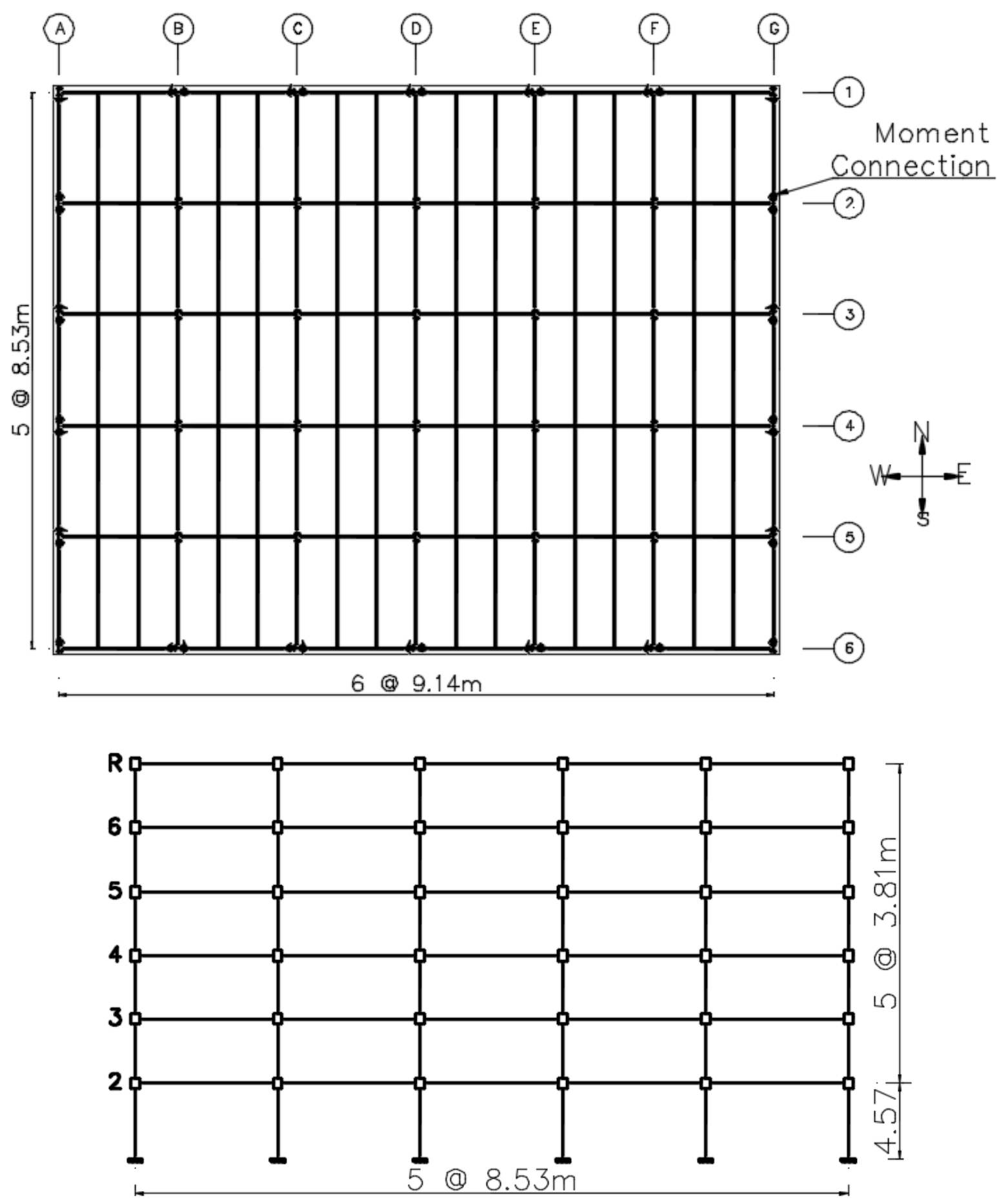

Figure 4-1. Six-story steel special moment resisting frame: plan and elevation 


\subsubsection{Modeling of Steel MRF}

The analytical models for the six-story steel frame building and superelastic viscous device are developed in OpenSees (OpenSees, 2014), a finite element framework for nonlinear and dynamic analysis of structural systems. The plane frame elements of the structure are modeled as elastic beam-column elements with concentrated inelastic springs using the Ibarra-Krawinkler hysteretic model (Ibarra et al., 2005) with the bilinear hysteretic rules. Figure 4-2(a) and (b) illustrates the Modified Ibarra Krawinkler hysteretic model for monotonic and cyclic behavior, respectively. The model considers cyclic stiffness and strength deterioration of the plastic hinges. Moment-rotation and cyclic deterioration parameters are adopted from Lignos (Lignos and Krawinkler, 2007).

The yield stress of structural steel is assumed to be equal to $375 \mathrm{MPa}$. Panel zones with doubler plates of beam-column joints are modeled using rotPanelZonet2D element, which is also known as Krawinkler model for panel zones (Lowes and Altoontash, 2003). The model includes four rigid links connected at the corners by four rotational springs. To consider the P-delta effects, leaning columns are connected to the plane frame with axially rigid truss elements at each story level. The model assumes Rayleigh damping with a $2 \%$ damping ratio for the first and third modes.

\subsubsection{Modeling of SVD}

The model of the superelastic viscous damper is developed in OpenSees by combining a finite length element with uniaxial self- centering material property to represent the SMAs and a zero-length element with the Maxwell material property to model the elastomeric compound. The self-centering material exhibits flag-shaped hysteric response and captures the post-transformation hardening behavior with a post-hardening stiffness equals to the initial stiffness. The experimental test results of SMAs and HD butyl compound reported in the literature (Silwal et al., 2015) are used to develop model parameters for the SMA and elastomeric compound. Since the SMAs will be subjected to dynamic loading rates during a seismic event, the material parameters for the SMA element are selected to match experimental response of the SMA cables at $1 \mathrm{~Hz}$. The selected parameters for the self-centering material model of the SMAs are as follows: initial stiffness $\mathrm{k}_{1}=$ $2.915 \mathrm{kN} / \mathrm{mm}$, post-activation stiffness $\mathrm{k}_{2}=0.994 \mathrm{kN} / \mathrm{mm}$, ratio of post-transformation hardening stiffness to initial stiffness $\gamma=1.0$, forward activation force $F_{a}=53.05 \mathrm{kN}$ and ratio of forward to 
reverse activation force $\beta=1.0$. The parameters for the Maxwell model is selected as follows: elastic spring coefficient $\mathrm{K}=1.43 \mathrm{kN} / \mathrm{mm}$, viscous damping coefficient $\mathrm{C}=1.00 \mathrm{kN}-\mathrm{s} / \mathrm{mm}$, nonlinear exponent coefficient $\alpha=0.8$. Figure 4-3 illustrates the parameters used to model SMA cables and butyl rubber in OpenSees.

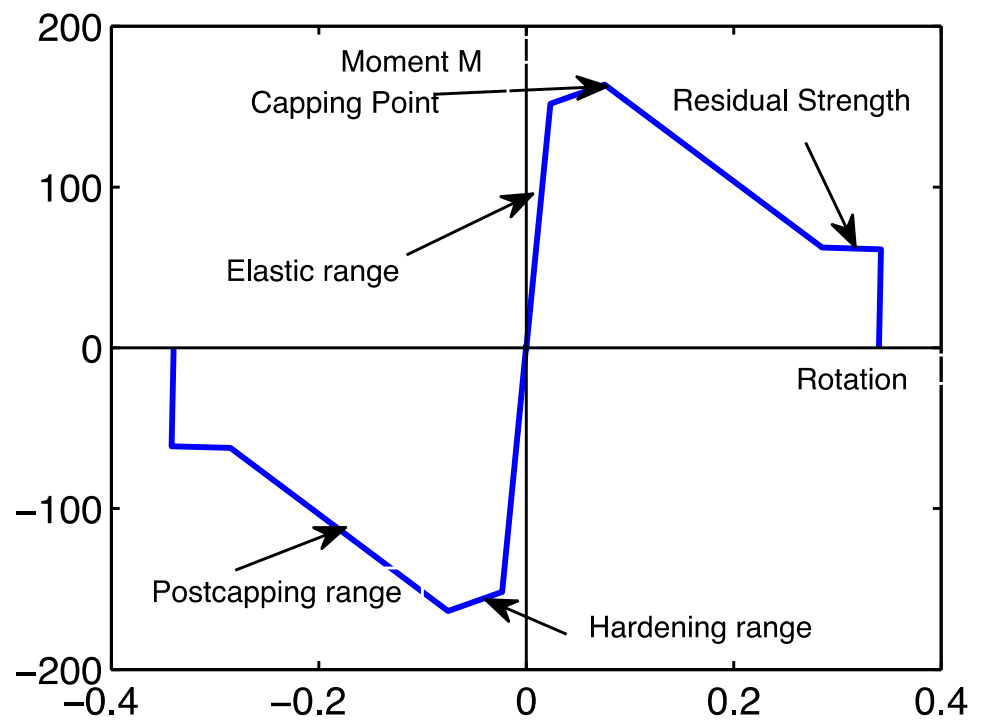

(a)

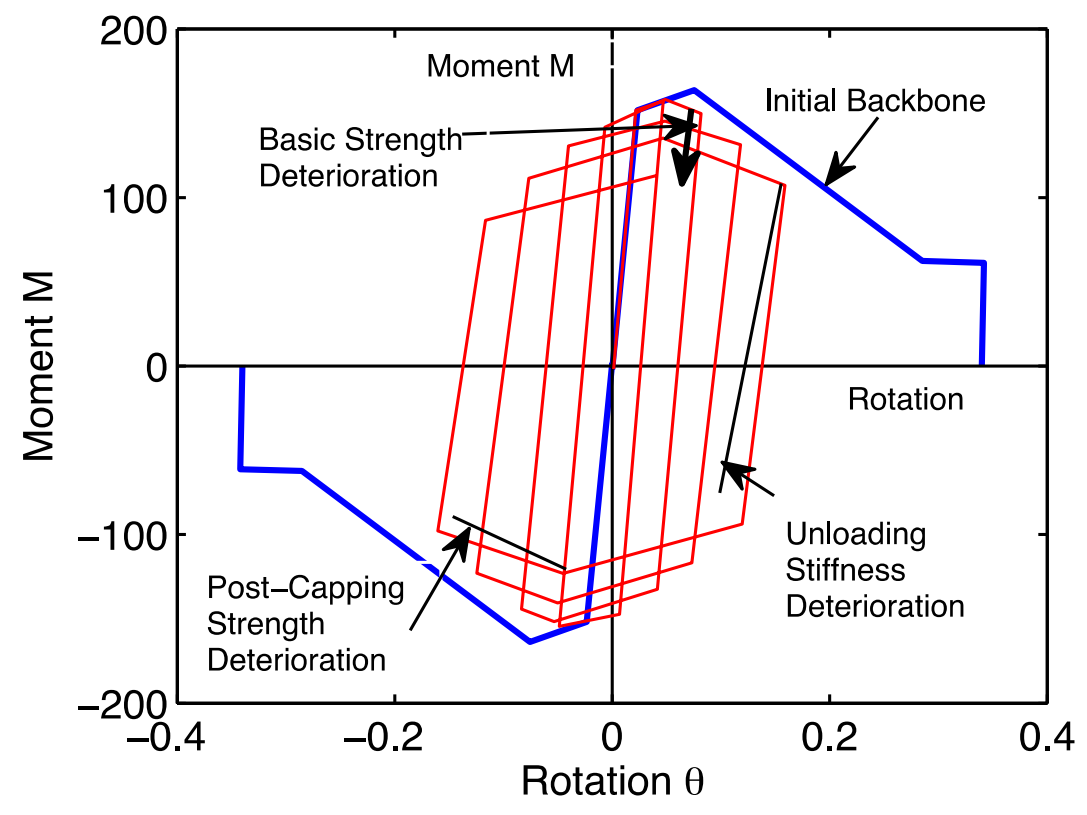

Figure 4-2 Modified Ibarra-Krawinkler Hysteretic Model: (a) monotonic and (b) cyclic 
Figure 4-4 illustrates the experimental stress-strain curve of SMA and VE components and the numerical model prediction. It can be seen that the models predict the response of NiTi SMA cables and butyl rubber reasonably well.
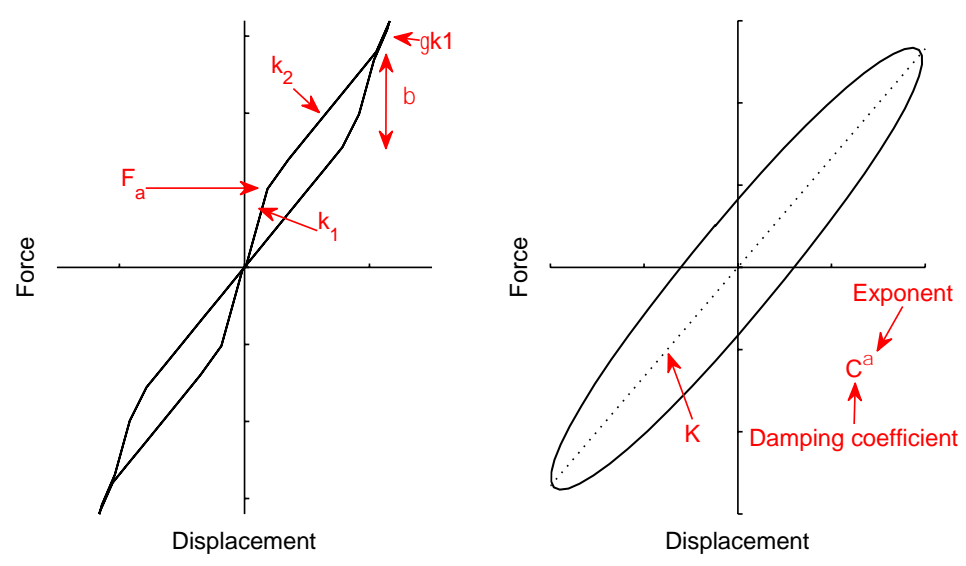

Figure 4-3. Parameters used to model SMA cables and butyl rubber in OpenSees

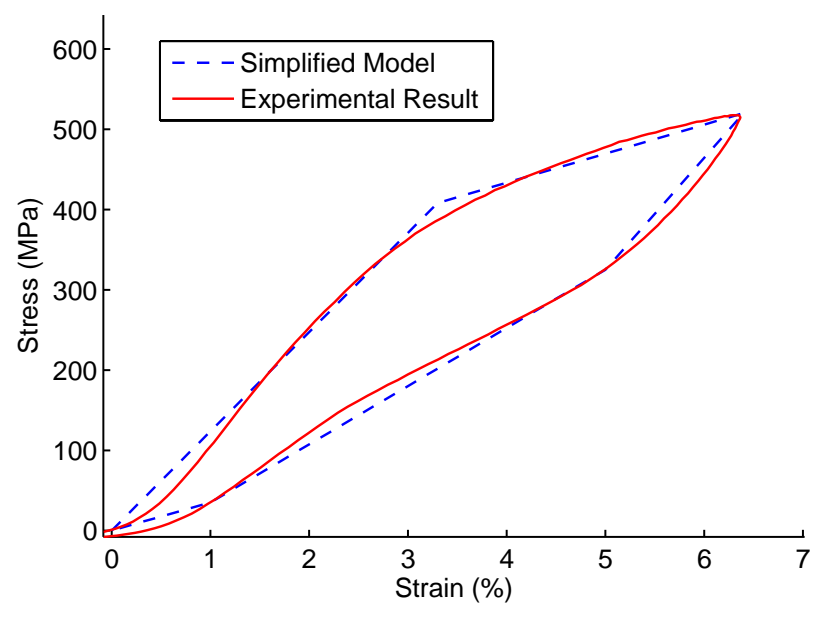

(a)

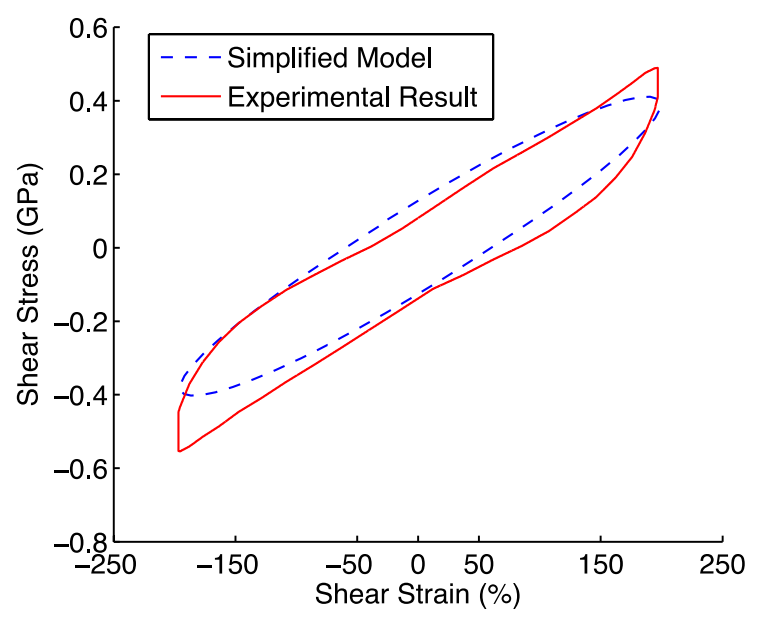

(b)

Figure 4-4. Experimental stress-strain curve and model prediction for (a) SMA cable, (b) VE device 


\subsubsection{Design of Steel MRF with SVDs}

Following the seismic design requirements for structures with damping systems described in Chapter 18 of ASCE/SEI 7-10, a nonlinear response-history procedure is used to design the steel frame with SVDs. Target spectra for design basis earthquake (DBE) and maximum considered earthquake (MCE) levels are developed in accordance with ASCE/SEI 7-10. The target design spectra are adjusted from 5\% damping to $2 \%$ damping using the modification factor given in ASCE 41 (ASCE, 2007). The modification factor used in this study is 0.815 . Figure $4-5$ illustrates $2 \%$ damped DBE and MCE level response spectra for Seismic Design Category (SDC) $\mathrm{D}_{\max }$. A total of 7 ground motions as shown in Table 4-1 are selected from PEER NGA database (PEER, 2014) and scaled according to ASCE/SEI 7-10 for the use in the response history analysis. In particular, the ground motions are scaled such that the average value of the response spectra for the selected ground motions is not less than the target response spectrum for periods ranging between 0.2 and 1.5 times the building fundamental period.

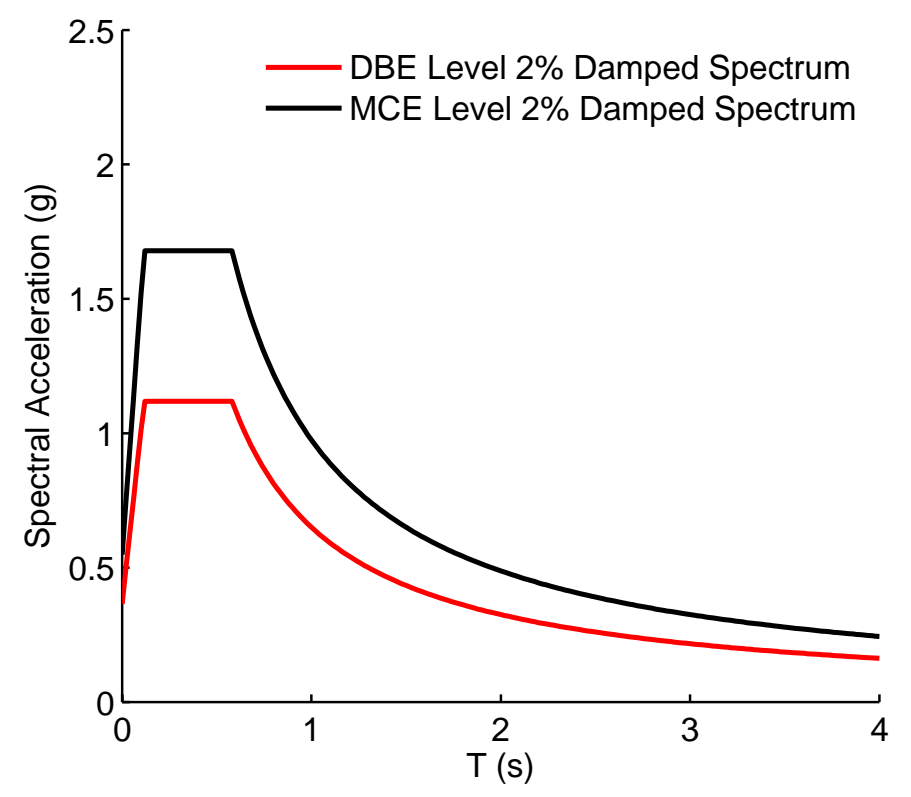

Figure 4-5. DBE and MCE level 2\% damped target response spectra 
The SVDs are installed at each bay at each story level. The design parameters described in Section 3 are used for the SVDs and these properties are set to be fixed for each device. The same numbers of dampers are assumed to be installed at each bay of a given floor. However, since the steel frame have larger drifts at lower stories, more dampers are used in lower stories. In particular, the number of control devices connected to the first and second stories, the third and fourth stories, and the fifth and sixth stories are selected to be 50, 40 and 30, respectively. The average response of the building with the installed dampers under the selected 7 ground motions satisfies the member strength criteria and 2\% drift requirements of the ASCE SEI 7-10. The fundamental period of the steel frame with the installed damper is 1.34 seconds.

Table 4-1 Seven ground motion records used in the design

\begin{tabular}{cccccc}
\hline No. & Earthquake & Station Name & $\begin{array}{c}\text { Magnitude } \\
\left(\mathrm{M}_{\mathrm{w}}\right)\end{array}$ & $\begin{array}{c}\text { Distance } \\
(\mathrm{km})\end{array}$ & $\begin{array}{c}\text { Peak Ground } \\
\text { Acceleration } \\
(\mathrm{g})\end{array}$ \\
\hline 1 & San Fernando (1971) & LA-Hollywood & 6.6 & 22.8 & 0.19 \\
2 & Imperial Valley-06 & Elcentro array & 6.5 & 12.6 & 0.37 \\
3 & Loma Prieta (1989) & Gilroy Array & 6.9 & 12.2 & 0.37 \\
4 & $\begin{array}{c}\text { Superstition Hills-02 } \\
(1987)\end{array}$ & El Centro Imp. & 6.5 & 18.2 & 0.26 \\
5 & Northridge (1994) & Canyon Country & 6.7 & 12.4 & 0.40 \\
6 & Duzce, Turkey (1999) & Duzce & 7.1 & 12.0 & 0.81 \\
7 & Kocaeli, Turkey (1999) & Bolu & 7.5 & 15.4 & 0.36 \\
\hline
\end{tabular}

\subsection{Ground Motions used for Analysis}

A total of 44 far-field ground motion records that are also used in the FEMA P695 (FEMA, 2009) methodology are employed for nonlinear time history analyses. The set includes strongmotion records, i.e. records with PGA $>0.2 \mathrm{~g}$ and $\mathrm{PGV}>15 \mathrm{~cm} / \mathrm{s}$, from event magnitudes range from M6.5 to M.7.6 and from stiff soil sites (site Class D) and very stiff soil sites (site Class C). The ground motions are normalized as described in FEMA P695 to eliminate unwarranted variability between records due to inherent differences in site conditions, source distance, source 
type, and event magnitude. Figure 4-6 shows acceleration spectra for individual records and the median response spectrum. The ground motion records are collectively scaled to selected hazard level, as defined in ASCE/SEI 7-05, such that the median spectral acceleration of the record set matches with those of the design spectra at the fundamental period of each frame (i.e. at 1.96 seconds for the uncontrolled frame and 1.34 seconds for the controlled frame).

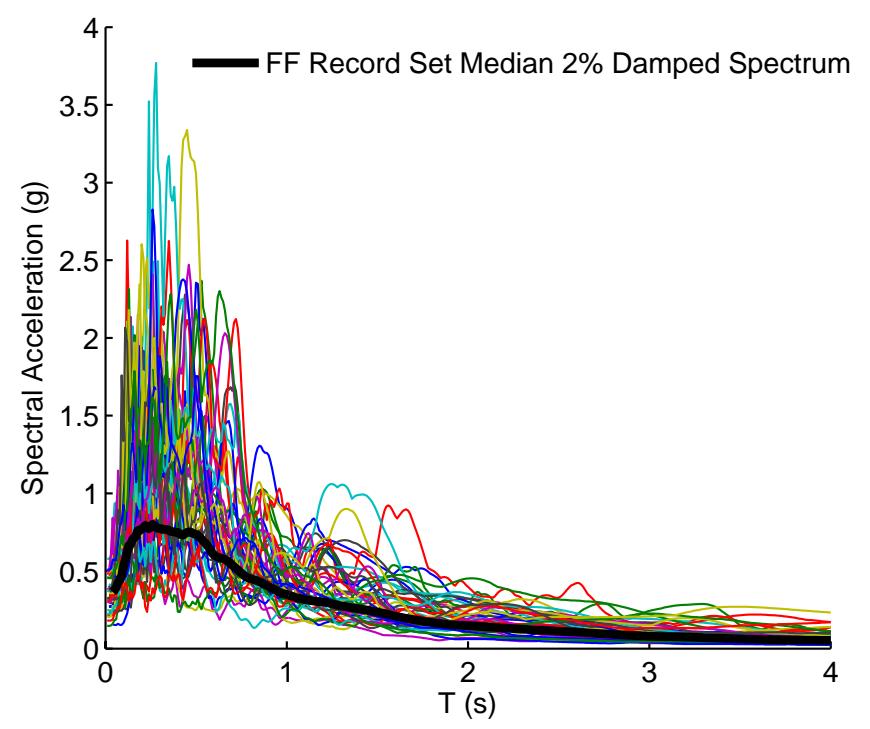

Figure 4-6. Acceleration spectra of individual ground motions for $2 \%$ damping and median spectrum

\subsection{Performance Assessment}

To evaluate efficacy of the SVDs in mitigating the seismic response of steel frame structures, extensive nonlinear response time history analyses are conducted for the conventional steel frame and the steel frame with SVDs. The response of steel frame with and without SVDs is evaluated under two different hazard levels: the design basis earthquake (DBE) seismic hazard level with a probability of exceedance of $10 \%$ in 50 years and the maximum considered earthquake (MCE) seismic hazard level with a probability of exceedance of $2 \%$ in 50 years.

Peak inter-story drift ratio, which is correlated with damage in structural elements; peak absolute floor acceleration, which is correlated with damage in non-structural components; and 
peak residual story drift ratio, which is related to the post-earthquake functionality of the structure are selected as the desired response quantities. Residual story drifts are calculated by continuing the analyses for at least 20 seconds after the end of the seismic event. As previous probability based studies suggested $10 \%$ inter-story drift ratio is considered to define the collapse in numerical simulations (Ribeiro et al., 2014).

Structural responses of the uncontrolled building and the building with installed hybrid dampers are computed under each ground motion record at two seismic hazard levels. Figures 47 and 4-8 illustrate the peak inter-story drift ratio, peak residual inter-story drift ratio, and peak floor absolute acceleration for the uncontrolled and controlled buildings under DBE and MCE level ground motion records, respectively. It can be seen that the installed SVDs reduces the drift demand of the six-story building under all DBE level earthquakes. For uncontrolled structure, the failure of the building is observed under nine ground motion records at the DBE level. For the controlled structure, the peak inter-story drift ratio is reduced below $3.1 \%$ during the same ground motions except one case where the peak inter-story drift ratio is recorded to be $6.2 \%$. In addition to collapse of the structure for nine records, residual story drift over $1 \%$ occurred in the uncontrolled building for eight other earthquake cases. Almost no residual drifts are observed for the controlled building for all ground motion records. However, peak story accelerations are slightly increased for the controlled building in most of the cases.

For MCE level seismic hazard, the number of cases where the structure failed is increased to seventeen for the uncontrolled building, whereas the collapse is observed only once for the controlled building. It can be seen that the uncontrolled building experience large permanent drifts for another eleven ground motion cases. On the other hand, the building upgraded with SVDs has minimal residual drifts for most cases. Similar to DBE level hazard, the controlled structure has higher acceleration response for more than half of the ground motion record cases. 

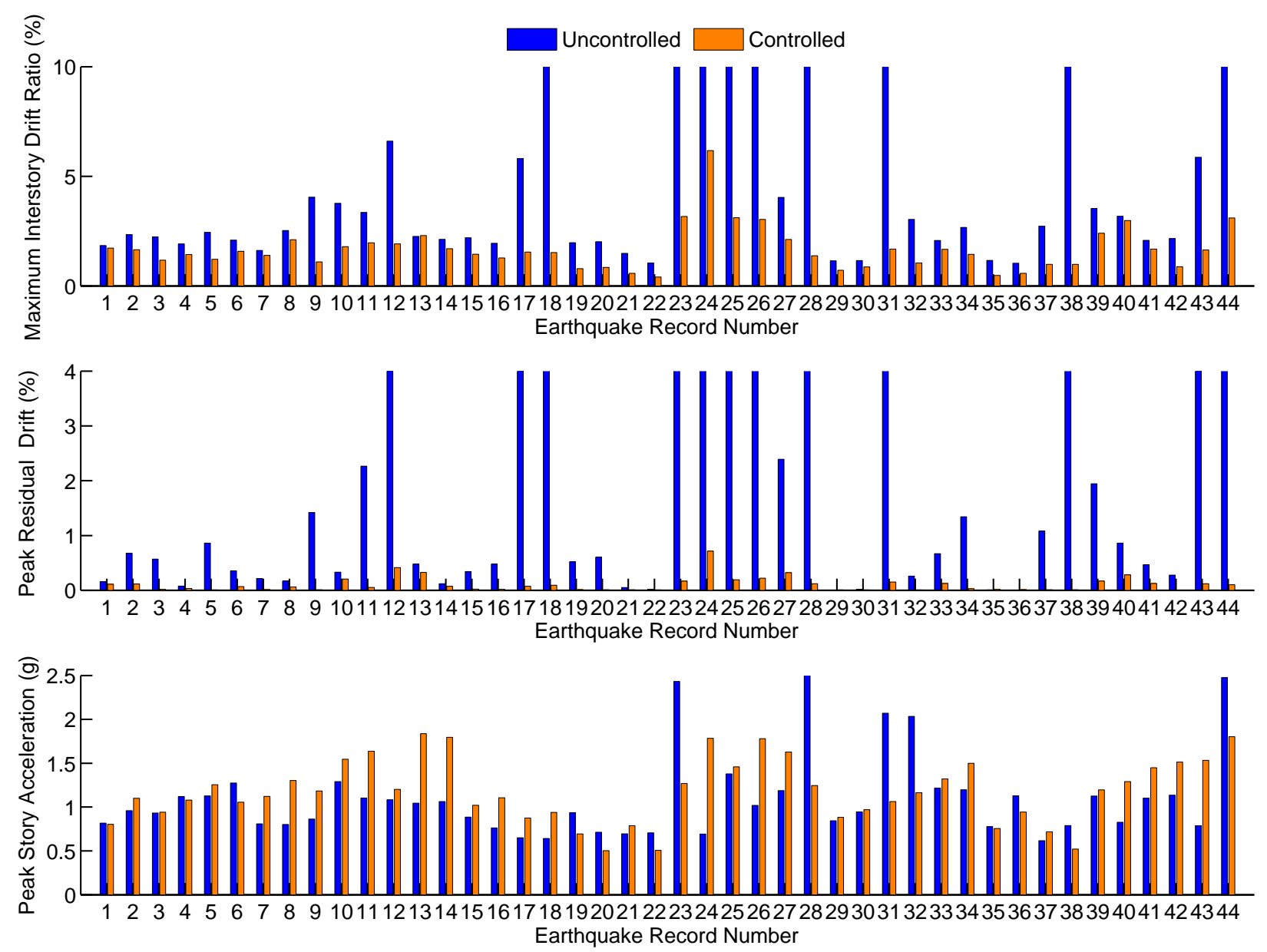

Figure 4-7. Peak inter-story drift, peak residual story drift, and peak story acceleration for individual DBE level ground motions

As the distribution of seismic response is typically assumed to follow a lognormal distribution, the statistics of the peak inter-story drift ratio and peak story absolute acceleration response under 44 ground motion records are provided in terms of median (50 percentile) and 84 percentile response and calculated as: 


$$
\begin{aligned}
& \hat{X}=\exp \left(\frac{\sum_{i=1}^{n} \ln x_{i}}{n}\right) \\
& X^{84}=\mu \exp \left(\sigma_{\ln X}\right)
\end{aligned}
$$

where $\mathrm{n}$ is the number of response data points and $\sigma_{\ln x}$ is the standard deviation of the logarithm of response $\mathrm{X}$.
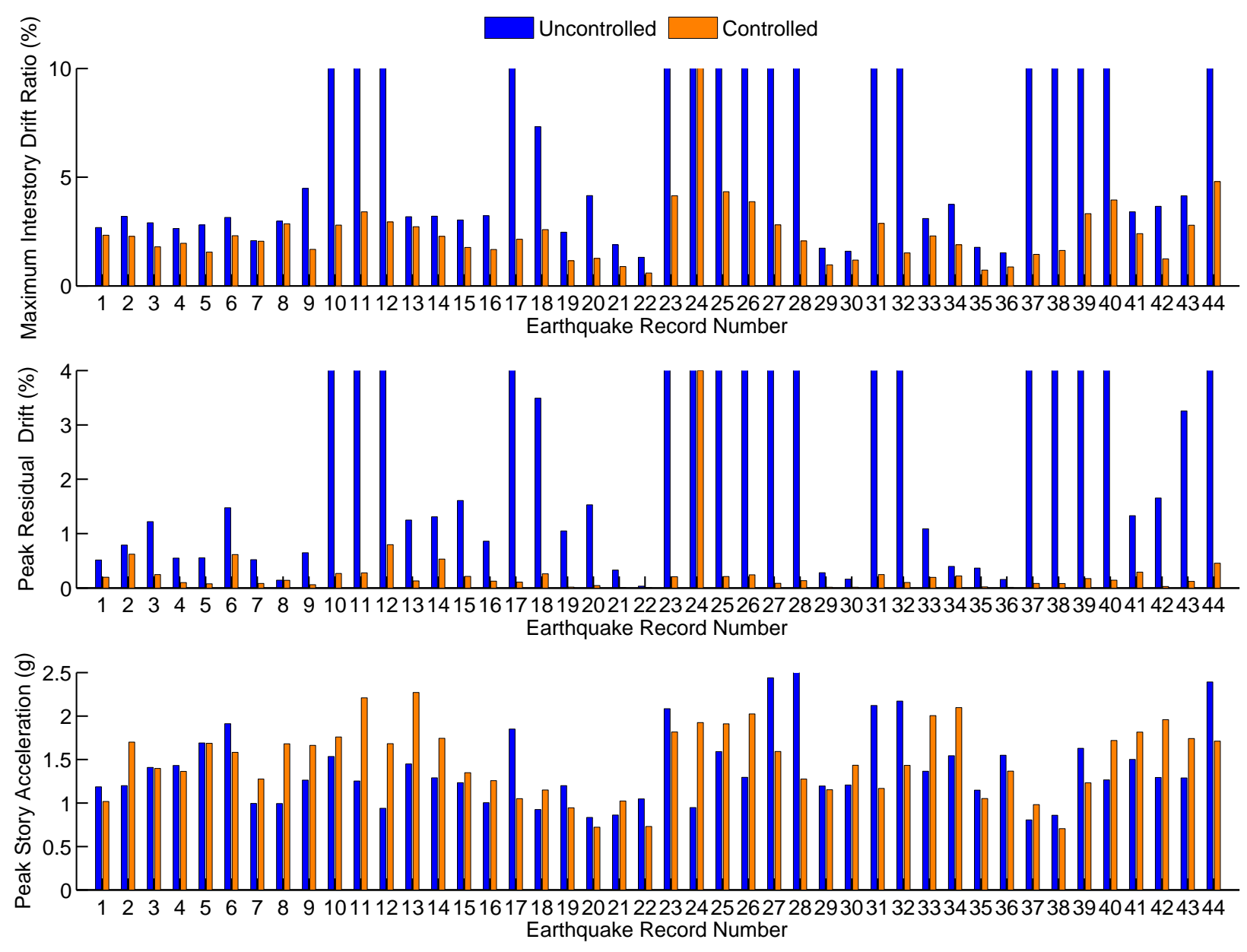

Figure 4-8. Peak inter-story drift, peak residual story drift, and peak story acceleration for individual MCE level ground motions 
Figure 4-9 shows the median and 84-percentile values of the peak inter-story drift ratio and peak story absolute acceleration at each floor level for the uncontrolled and controlled buildings to the DBE and MCE levels. It can be seen that peak inter-story drift attain smaller values for the controlled structure at all the floors for both DBE and MCE hazard levels. The median peak interstory drift ratio reaches about $2.8 \%$ and $4 \%$ for bottom three floors for the uncontrolled structure for the DBE and MCE levels, respectively. On the other hand, the median peak inter-story drift ratio is uniformly distributed along the height of the structure for the controlled frame with a maximum of $1.3 \%$ and $1.8 \%$ for the DBE and MCE levels, respectively. That clearly shows the effectiveness of SVDs in reducing the displacement response of the steel building, especially at high seismic hazard levels. Furthermore, the maximum 84-percentile inter-story drift ratios for the conventional frame are $6.5 \%$ and $9.0 \%$ for the DBE and MCE, respectively. The corresponding values are only $2.3 \%$ and $3.3 \%$ for the steel frame with SVDs. The peak acceleration response slightly increases for the steel frame with the installed dampers. However, the increase in the median peak acceleration is only $22 \%$ for the DBE level and $15 \%$ for the MCE level. The peak median accelerations for the uncontrolled frame are $0.88 \mathrm{~g}$ and $1.16 \mathrm{~g}$ and the peak 84-percentile acceleration values are $1.34 \mathrm{~g}$ and $1.62 \mathrm{~g}$ for the DBE and MCE, respectively. For the controlled frame, the median values are $1.08 \mathrm{~g}$ and $1.33 \mathrm{~g}$ at DBE and the peak 84-percentile acceleration values are $1.51 \mathrm{~g}$ and $1.80 \mathrm{~g}$ at MCE.

Figures 4-10 and 4-11 compare the time histories of the inter-story drift for the first floor and absolute acceleration for the top floor of the uncontrolled and controlled structures subjected to 1994 Northridge (ground motion \# 1) and 1995 Kobe earthquakes (ground motion \# 15) scaled to MCE level. These figures also include the force-displacement curves of the subcomponents of the SVD and combined hysteresis for one of the SVDs installed into the first floor. It can be seen that the drift response of the controlled frame under Kobe earthquake has reduced number and magnitude excursions with zero permanent deformations, while the uncontrolled structure experience considerable residual drift due to inelastic deformations. Although the controlled frame experienced larger drifts than the uncontrolled frame for the Northridge earthquake, it does not have considerable permanent drifts at the end of seismic event. Also, the vibrations cease much earlier for the controlled frame. 

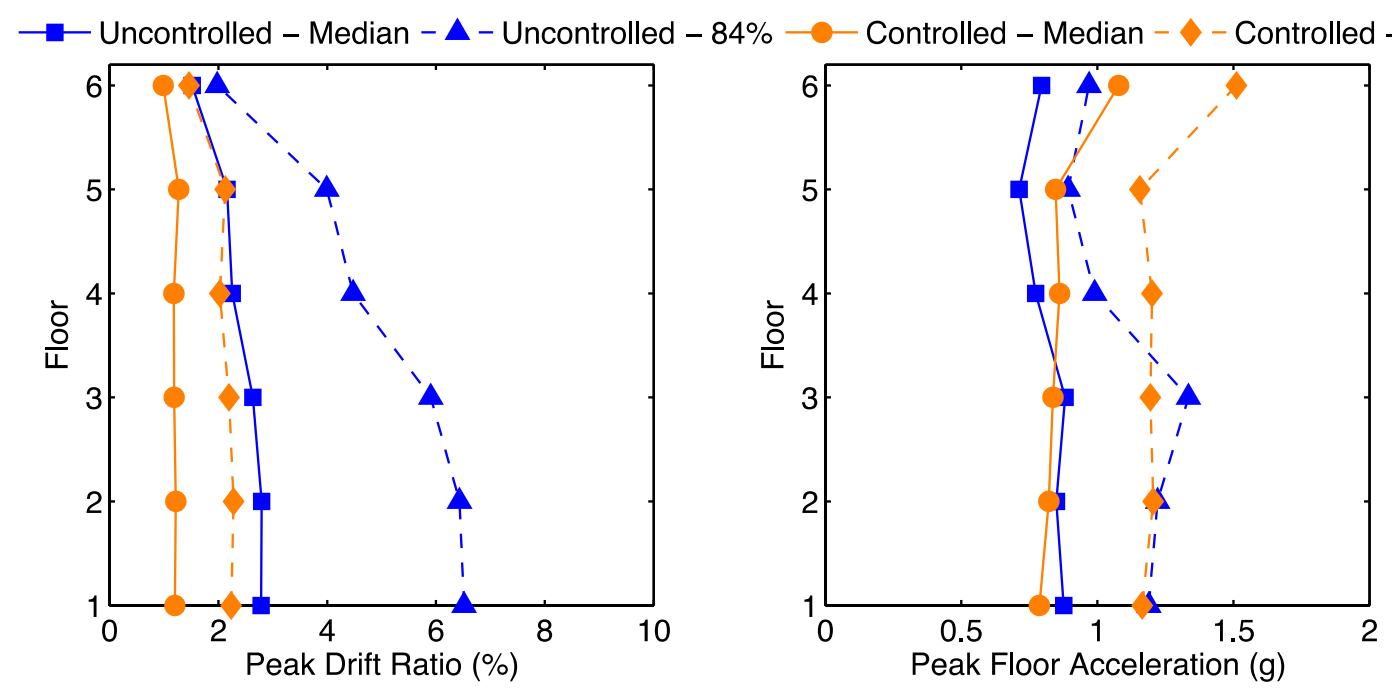

(a)
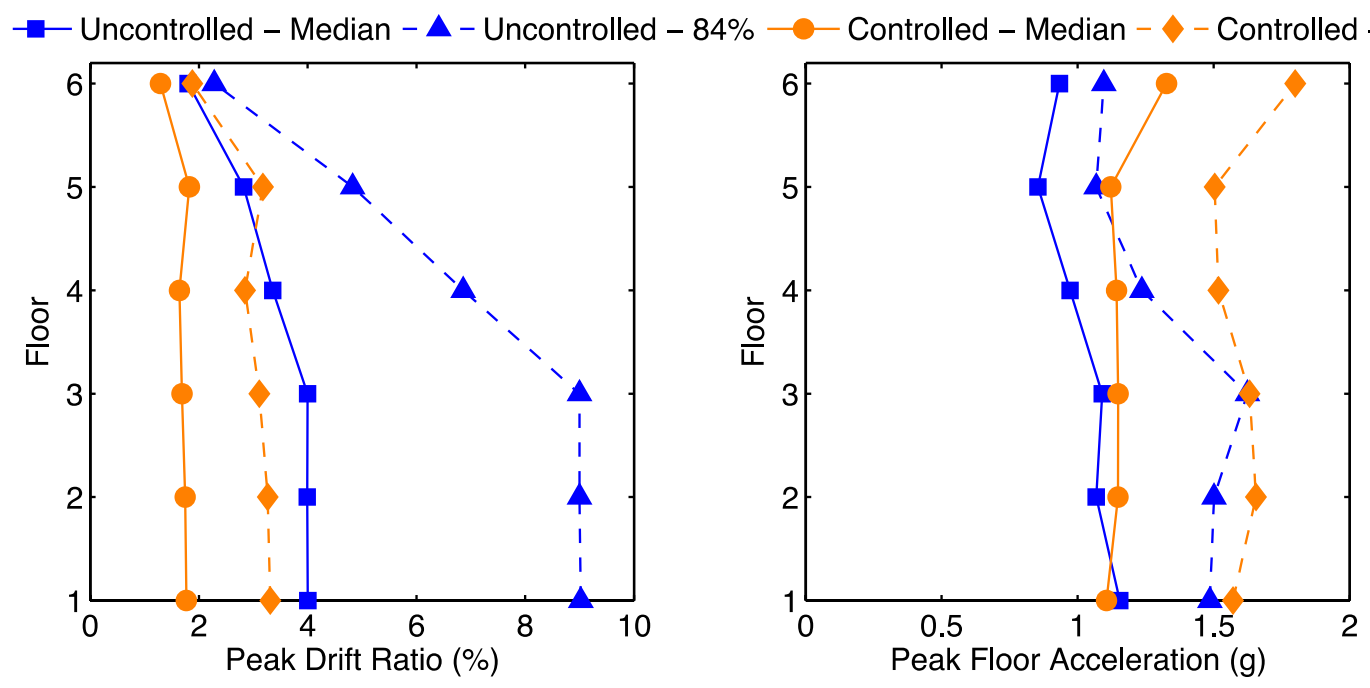

(b)

Figure 4-9. Statistics of peak inter-story drift ratio and peak story absolute acceleration for uncontrolled and controlled buildings subjected to 44 ground motions at (a) DBE level and (b) MCE level 

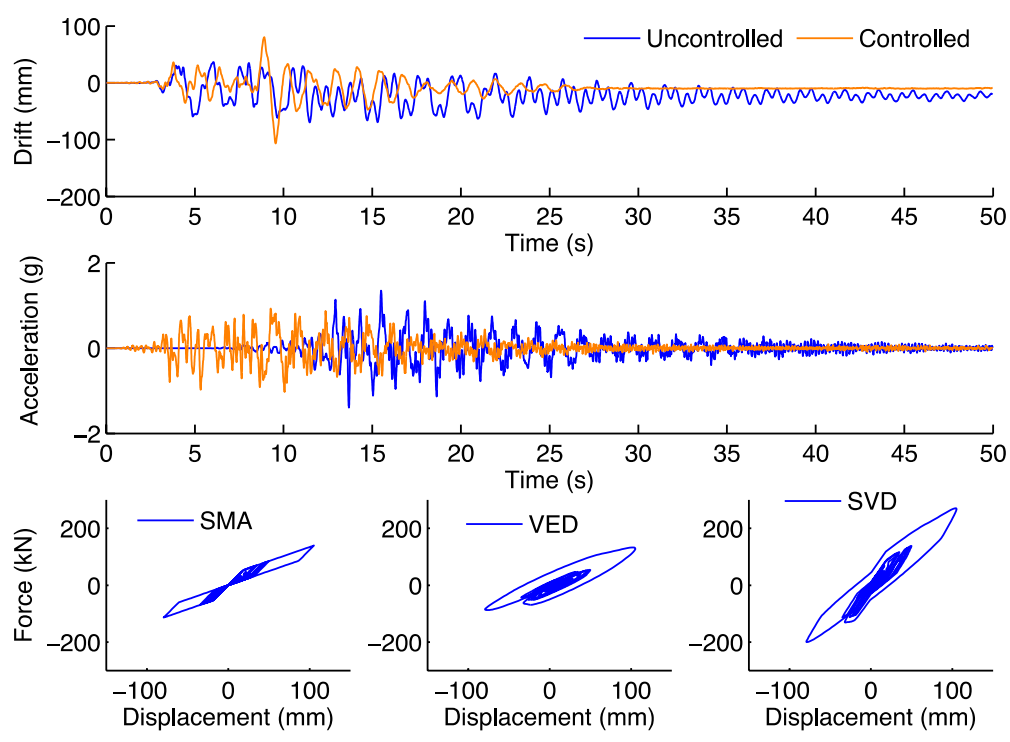

Figure 4-10. Time histories of selected response for uncontrolled and controlled structures subjected to MCE level Northridge earthquake and corresponding force-deformation curves for SVD and its subcomponents
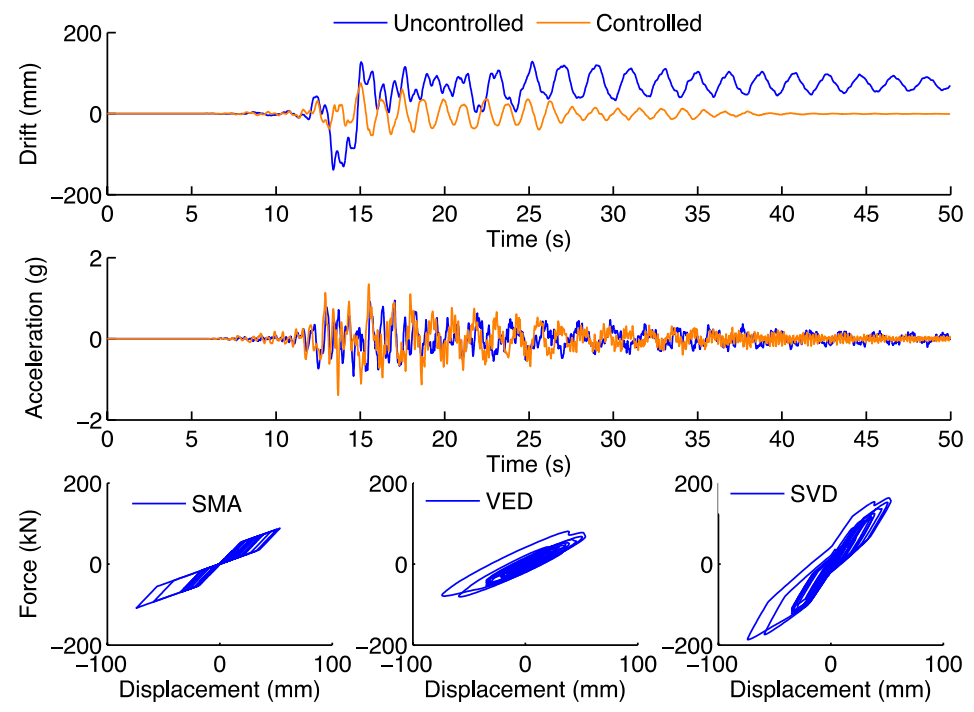

Figure 4-11. Time histories of selected response for uncontrolled and controlled structures subjected to MCE level Kobe earthquake and corresponding force-deformation curves for SVD and its subcomponents 


\subsection{Closure}

The performance and effectiveness of the proposed SVD in mitigating the response of steel frame buildings under DBE and MCE level seismic loads are assessed through numerical studies. A six-story special steel moment frame structure is modeled as a conventional moment resisting frame and a frame with installed superelastic viscous dampers. A suite of 44 strong ground motion records are normalized and scaled as described in the FEMA P695. Nonlinear response history analyses are conducted and the peak response quantities are evaluated. Results show that the interstory drift demands and residual drifts of the buildings subjected to DBE and MCE level

earthquakes can significantly be reduced with the installed SVDs without a considerable increase in peak acceleration demand. 


\section{SEISMIC COLLAPSE ASSESSMENT OF STEEL FRAME BUILDINGS WITH SVDS}

\subsection{Introduction}

This section investigates the seismic collapse resistance of steel moment resisting frames upgraded with the superelastic viscous damper (SVD) through incremental dynamic analysis (IDA). First, a nine-story steel frame building is designed as a conventional special moment resisting frame (SMRF) to meet the current seismic design requirements. Next, the same building is designed with SVDs to achieve a performance similar to that of conventional SMRF. For this purpose, a reduced strength version of the fully code-compliant frame is developed. In particular, the beam and column sizes are reduced such that the steel frame satisfies the strength requirements of the design codes but does not meet the drift limits. In this more flexible frame, the added dampers carry a large portion of the seismic loads and are mainly responsible to control the story drift. For comparison purposes, the steel building with the reduced strength is also designed with buckling restrained braces (BRB). The reduced strength frame is also designed with BRBs to meet the story drift requirements according to the ASCE 7-10. A typical BRB consists of three components: a central core designed to yield in tension and compression, buckling restrained elastic transition zone, and unrestrained elastic zone.

Incremental dynamic analyses are conducted using 44 ground motion records to assess the collapse resistance of each frame. The results are analyzed in terms of peak inter-story drift, peak floor absolute acceleration, and peak residual drifts. Finally, a sensitivity analysis is also performed to evaluate the influence of SVD design parameters on the overall seismic response of the steel frame designed with SVDs.

\subsection{Design of Model Buildings}

\subsubsection{Steel Moment Resisting Frame}

A nine-story steel building (SAC, 2000) is selected from the SAC steel project for numerical analyses. The selected building includes a basement level in addition to the nine stories above the ground level and was originally designed as an office building located on a stiff soil site 
(Site Class D) in Seattle, Washington. A floor plan and elevation of the nine-story building are

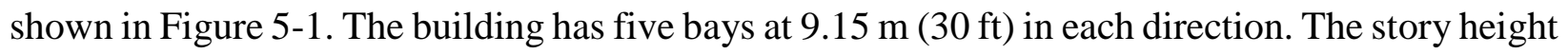

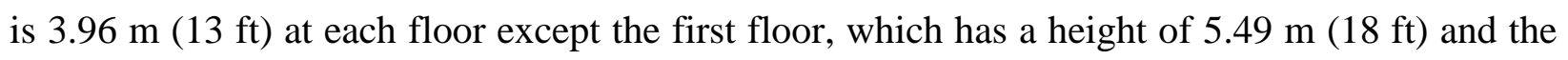
basement, which has a height of $3.65 \mathrm{~m}(12 \mathrm{ft})$. The lateral load-resisting system in both directions consists of two special moment resisting frames on the perimeter of the building. This study analyzes one of the moment frames in the E-W direction. The seismic masses are assigned as 1.01 $\times 10^{6} \mathrm{~kg}$ for floor level $2,9.89 \times 10^{5} \mathrm{~kg}$ for floor levels $3-9$, and $1.07 \times 10^{6} \mathrm{~kg}$ for roof level. All the columns are assumed to be pinned at the base. The exterior columns at the ground level are also restrained laterally.

The OpenSees is utilized to develop the analytical model of the nine-story steel frame building as a two-dimensional plane frame with panel zone and reduced beam section. To capture the structural behavior up to the collapse, the steel moment resisting frame is modeled as the nonlinear 2D frame capable of simulating different modes of strength and stiffness degradation associated with structural damages during earthquake events. Based on the concentrated plasticity concept, the flexural behavior of beam and column elements are modeled with elastic beamcolumn elements connected by zero-length inelastic rotational springs. To this end, a bilinear hysteretic material based on the modified Ibarra-Krawinkler deterioration model (Ibarra et al. 2005) that is capable of simulating flexural strength and stiffness deterioration of the material is used for the plastic hinges. Figure 4-2 illustrates the modified Ibarra- Krawinkler model, which is characterized by the yield point, the capping point, residual point, and ultimate point. Figure 5-2 displays the schematic numerical model of the partial steel frame in OpenSees. Here, model parameters are determined from Lignos and Krawinkler (Lignos and Krawinkler, 2007), which provides empirical equations from the calibration of experimentally tested steel section specimens. 

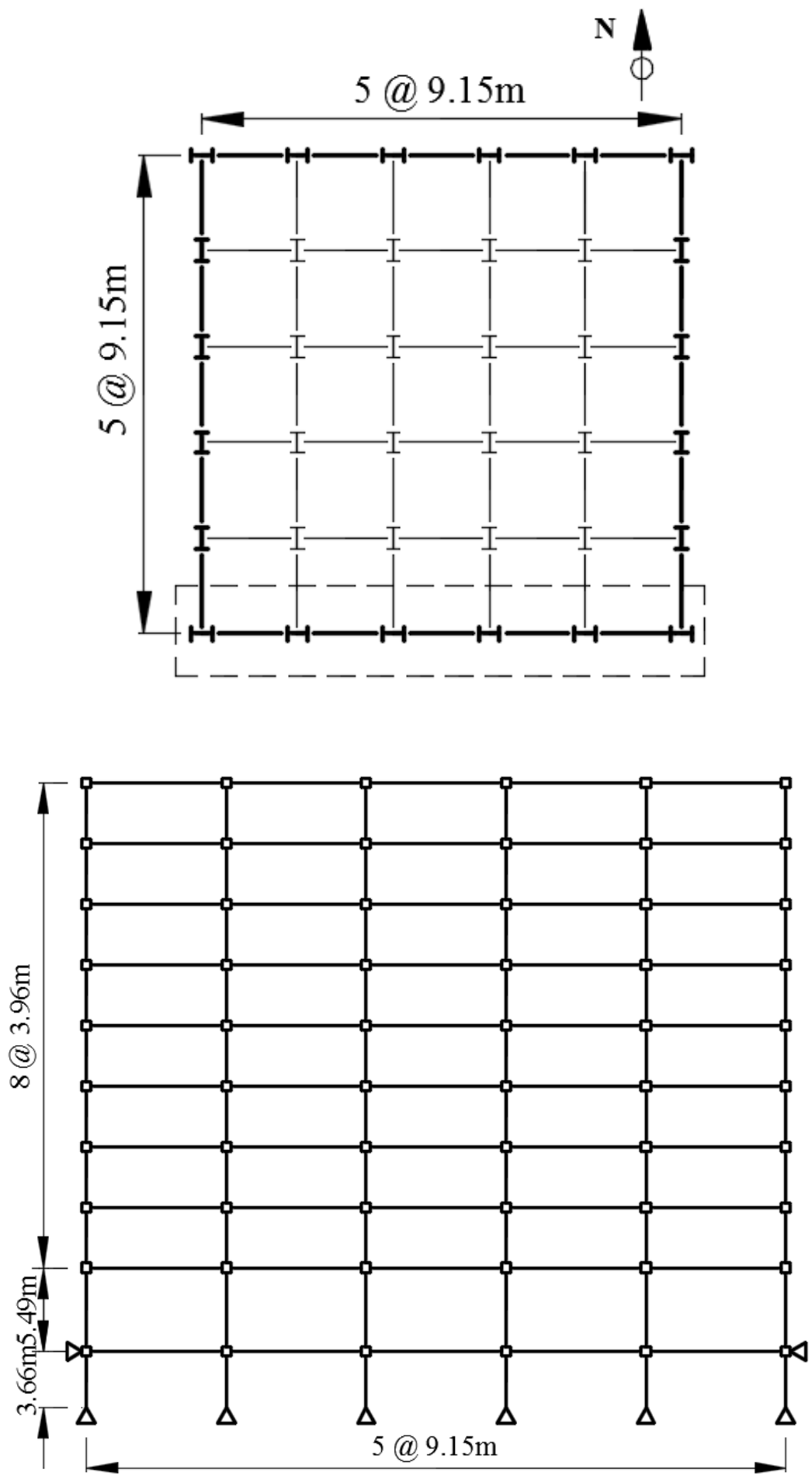

Figure 5-1. Plan and elevation of nine-story steel special moment resisting frame 
To capture shear yielding in the panel zone, the panel zone is modeled using eight very stiff elastic beam-column elements arranged as a rectangle and one zero-length rotational spring at the upper right corner to represent the shear distortion in the panel zone (Gupta and Krawinkler, 1999). The nominal yield strengths of beam and column elements are assigned to be $250 \mathrm{MPa}$ and $345 \mathrm{MPa}$, respectively. To simulate the P-delta effects, a leaning column carrying gravity loads is also created with elastic beam-column elements connected by zero-length rotational spring elements with negligible stiffness and is linked to the plane frame model with axially rigid truss element at each story level as shown in Figure 5-2. Rayleigh damping with a 2\% damping ratio for the first and third modes is assigned to the model to capture the inherent damping.

The building is designed according to ASCE 7-10 (ASCE, 2010) as a Risk Category II building by using nonlinear response history procedure. Based on the Seismic Design Category (SDC) D, the following design response spectral values are assigned to the site: $\mathrm{S}_{\mathrm{DS}}=0.912 \mathrm{~g}$ and $\mathrm{S}_{\mathrm{Dl}}=0.530 \mathrm{~g}$ for the design basic earthquake (DBE), and $\mathrm{S}_{\mathrm{MS}}=1.368 \mathrm{~g}$ and $\mathrm{S}_{\mathrm{Ml}}=0.795 \mathrm{~g}$ for the maximum considered earthquake (MCE). Using the site's spectral acceleration values, the target spectra for the DBE and MCE levels are developed. A total of 7 ground motions is selected from PEER NGA database (PEER, 2014) as shown in Table 4-1 and scaled according to ASCE 7-10. In particular, the ground motions are scaled such that the average response spectra for the selected records is not less than the target response spectrum for periods ranging between 0.2 and 1.5 times the building fundamental period. The steel members of the nine-story building are selected in accordance with the strength requirements of ANSI/AISC 360-10 (AISC, 2010) under the load combinations provided in ASCE 7-10. The building is also designed to comply with the drift requirements of ASCE 7-10. Since a nonlinear response history is adopted for the design, the allowable story drift is increased by $25 \%$ and determined from ASCE 7-10 for Risk Category II buildings as $2.5 \%$ under DBE level and as 3.75\% under MCE level. The selected column and beam sections for the nine-story frame are shown in Table 5-1. The building satisfies the drift requirements under both DBE and MCE levels as will be illustrated below. The first mode period of the special moment resisting frame is 2.44 seconds. 


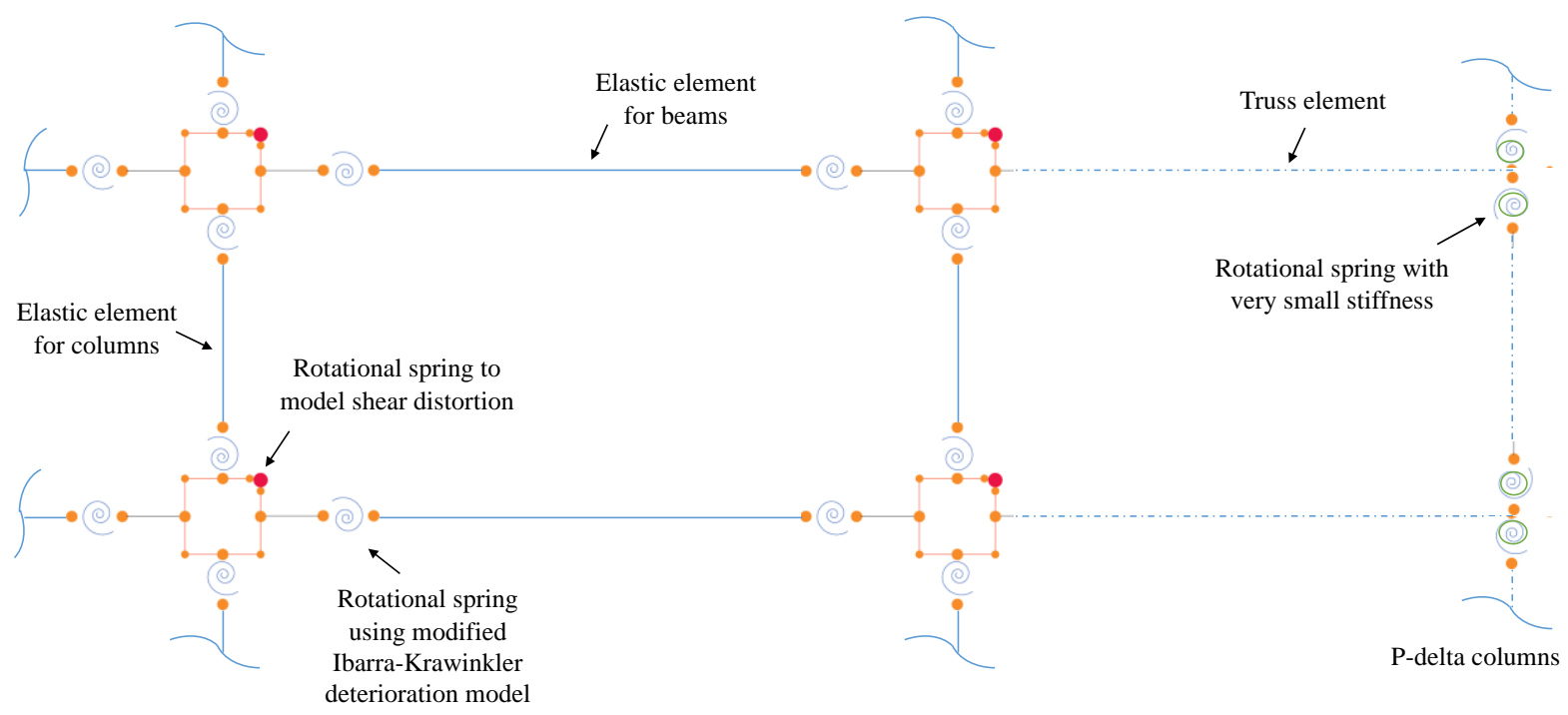

Figure 5-2 Schematic numerical model of the partial steel frame in OpenSees

\subsubsection{Steel Moment Frame with SVDs}

In order to design the nine-story frame with damping systems, a reduced strength version of the fully code-compliant frame is developed first. In particular, the beam and column member sizes are reduced such that the steel frame satisfies the strength requirements of the design codes but does not meet the drift limits. In this more flexible frame, the added dampers will carry a larger portion of the seismic loads and will be mainly responsible to control the story drifts. Table5-1 provides the selected member sizes for the reduced strength frame. The reduced strength steel frame is first upgraded with SVDs to comply with the story drift requirements of ASCE 7-10. In particular, the steel frame with SVDs is designed using the nonlinear response history procedure and following the seismic design requirements for structures with damping systems described in Chapter 18 of ASCE 7-10. The SVDs are installed at the second and fourth bay of each story level using a chevron brace configuration shown in Figure 5-3. The SVDs are modeled in OpenSees as described in Section 4.2.3. From the nonlinear time history analyses, the number of dampers for each story is selected to be 8 to meet the drift requirements. The fundamental period of the frame with the installed SVDs is 2.24 seconds. 
Table 5-1 Members of steel moment resisting frames

\begin{tabular}{ccccccc}
\hline & \multicolumn{2}{c}{ SMRF } & \multicolumn{2}{c}{ SMRF with SVD } & \multicolumn{2}{c}{ SMRF with BRB } \\
\cline { 2 - 7 } Story & $\begin{array}{c}\text { Exterior/ } \\
\text { Interior } \\
\text { Columns }\end{array}$ & Girders & $\begin{array}{c}\text { Exterior/ } \\
\text { Interior } \\
\text { Columns }\end{array}$ & Girders & $\begin{array}{c}\text { Exterior/ } \\
\text { Interior } \\
\text { Columns }\end{array}$ & Girders \\
\hline 1 & W18x311 & W21x201 & W18x311 & W21x201 & W18x311 & W21x201 \\
2 & W18x311 & W21x201 & W18x311 & W21x201 & W18x311 & W21x201 \\
3 & W18x311 & W21x201 & W18x283 & W21x182 & W18x258 & W21x166 \\
4 & W18x311 & W21x201 & W18x283 & W21x182 & W18x258 & W21x166 \\
5 & W18x283 & W21x182 & W18x234 & W18x192 & W18x211 & W21x132 \\
6 & W18x283 & W21x182 & W18x234 & W18x192 & W18x211 & W21x132 \\
7 & W18x234 & W18x192 & W18x192 & W18x143 & W18x143 & W18x106 \\
8 & W18x234 & W18x192 & W18x192 & W18x143 & W18x143 & W18x106 \\
9 & W18x192 & W18x175 & W18x143 & W18x130 & W18x86 & W18x65 \\
R & W18x192 & W18x175 & W18x143 & W18x130 & W18x86 & W18x65 \\
\hline
\end{tabular}

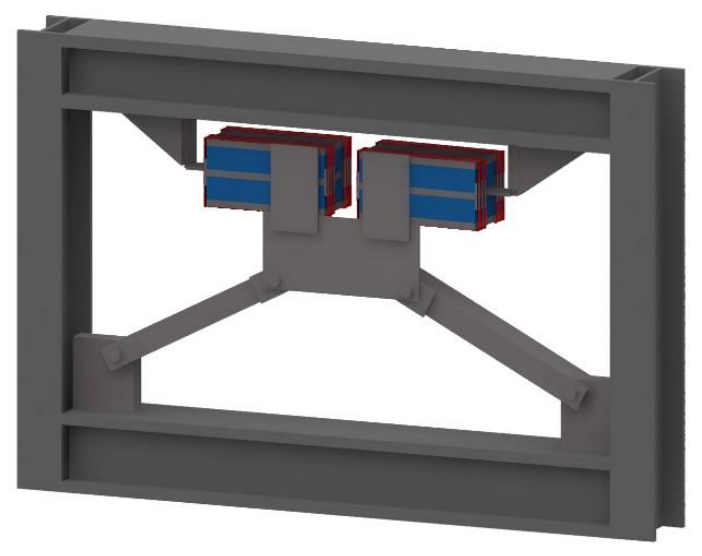

Figure 5-3. Superelastic viscous dampers installed into steel frame 


\subsubsection{Steel Moment Frame with BRBs}

The reduced strength frame discussed above is also upgraded with the buckling restrained braces (BRBs) to meet the story drift requirements according to ASCE 7-10. BRB is a structural component that exhibits a ductile load-deformation behavior under both tension and compression and a high-energy absorption capacity. A typical BRB consists of a central core designed to yield in tension and compression, buckling restrained elastic transition zone, and unrestrained elastic end zone as shown in Figure 5-4. Buckling-restraining mechanism is typically composed of mortar and steel tube casing. The area of the central core section is lower than that of the transition and end zones to provide a controlled yielding to the core segment. Here, the BRB elements are modeled in OpenSees using an inelastic corotTruss element that resists only axial force and deformation. To represent the overall hysteric behavior of a BRB that includes a variable crosssectional area along the length of the brace, a simple model with a constant cross-sectional area is assigned for the entire length of the truss element. In the model, the elastic stiffness and the yield strengths are adjusted to consider the effect of the BRB area variation to the response of the brace. The elastic stiffness of the BRB is modified as described in Oxborrow (Oxborrow, 2009) to represent the equivalent stiffness and, the yield strength of the BRB is adjusted as discussed in Coy (Coy, 2007) to match the hysteric response with experimental results. The material behavior for he $\mathrm{BRB}$ is represented using a steel02 material, which is a bilinear hysteric model that considers a uniaxial Giuffre-Menegotto-Pinto material behavior with isotropic strain hardening.

The nonlinear response history analysis procedure is adopted to design the BRB frame using the 7 ground motion records given in Table 4-1. The BRBs with the same capacity are installed in second and forth bay of each story using a diagonal brace configuration as shown in Figure 5-4(b). The yield stress of the BRB used in the design is $260 \mathrm{MPa}$ and the yield force capacity of the BRB is $405 \mathrm{kN}$. The first mode period of the nine-story frame upgraded with BRBs is 2.12 seconds. 

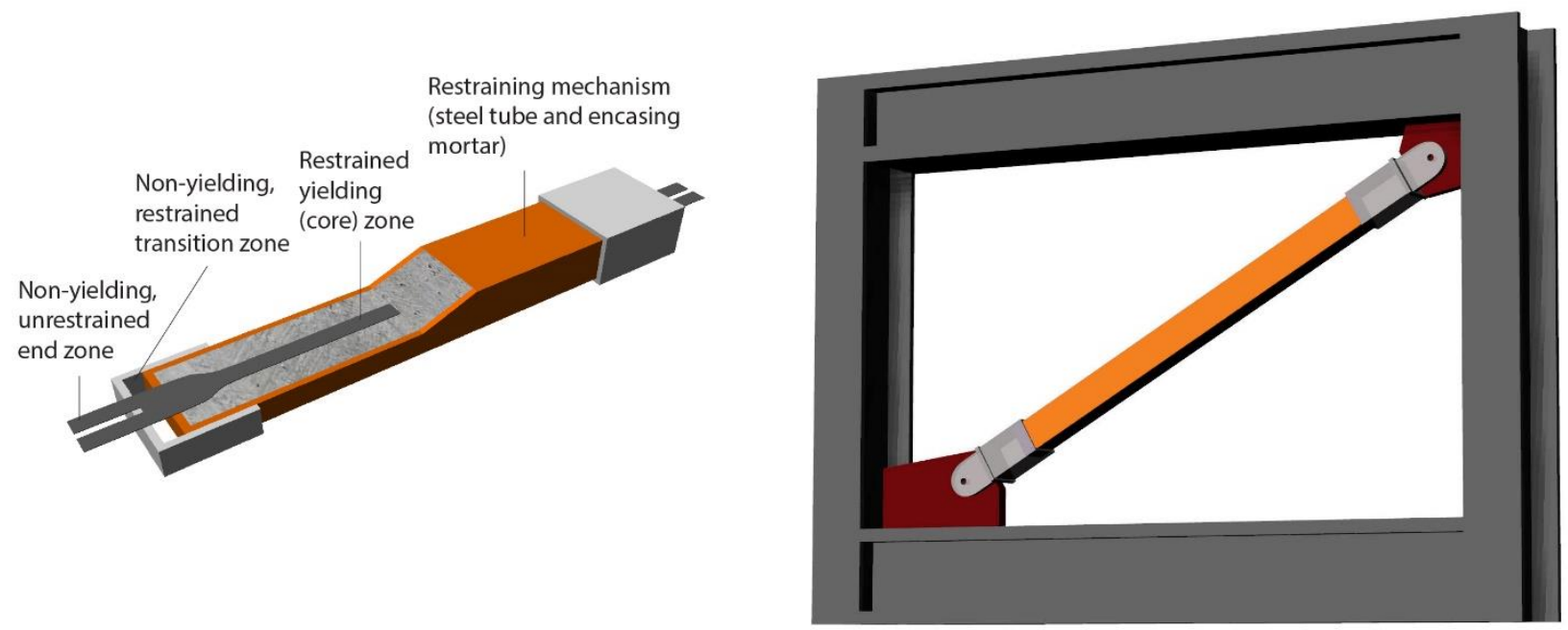

Figure 5-4. (a) Components of buckling restrained brace and (b) BRB installed to steel frame

The mean story drifts for the SMRF, the steel frame with SVDs and the steel frame with BRBs under seven ground motions are provided in Table 5-2 for the DBE and MCE levels. Note that each frame is designed such that the peak inter-story drift obtained from nonlinear response history procedure meets the code drift requirements without aiming to achieve any higher seismic performance objective. For each steel frame, it can be seen that the mean story drift response under DBE level earthquakes is similar while the SMRF has the lowest drift response under MCE level ground motions.

Table 5-2 Mean story drifts under 7 design ground motions at DBE and MCE level

\begin{tabular}{ccccc}
\hline \multirow{2}{*}{ Frame } & \multicolumn{2}{c}{ DBE } & \multicolumn{2}{c}{ MCE } \\
\cline { 2 - 5 } & Design & Limit & Design & Limit \\
\hline SMRF & $2.49 \%$ & $2.5 \%$ & $3.66 \%$ & $3.75 \%$ \\
SVD & $2.24 \%$ & $2.5 \%$ & $3.22 \%$ & $3.75 \%$ \\
BRB & $2.42 \%$ & $2.5 \%$ & $3.34 \%$ & $3.75 \%$ \\
\hline
\end{tabular}




\subsection{Performance Assessment}

\subsubsection{Ground Motion Records}

A set of 22 far-field ground motion pairs used in the FEMA P695 methodology (FEMA, 2009), which is described in Section 4.3, is employed in this study for extensive nonlinear time history analyses. The acceleration spectra of individual records and the median response spectrum are shown in Figure 5-5. The design spectra at DBE and MCE levels are also shown in the figure. The spectral acceleration at the fundamental period of SMRF, SVD, and BRB frames at the MCE seismic hazard level are found to be $0.33 \mathrm{~g}, 0.35 \mathrm{~g}$, and $0.38 \mathrm{~g}$, respectively.

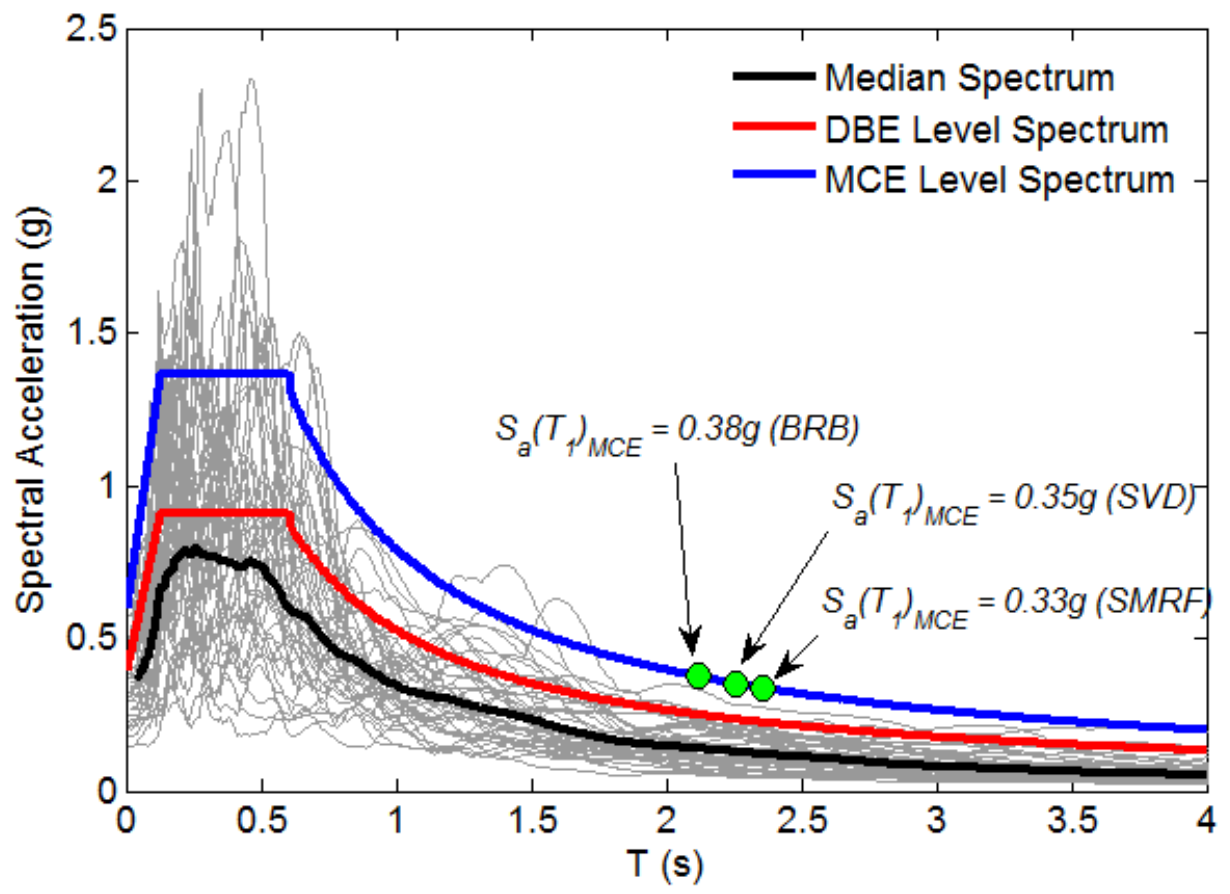

Figure 5-5. Acceleration spectra of each ground motion for 5\% damping and median spectrum

\subsubsection{Pushover Curves}

A displacement-controlled static pushover analysis is performed to evaluate the lateral strength and post-yield behavior of each steel frame system. The pushover analyses are conducted using a static lateral force distribution with a load pattern based on the first mode shape of the structure. Figure 5-6 shows the relationship between the base shear normalized by the weight of 
the structure $\left(V / W_{s}\right)$ and the roof drift ratio of each frame system. It can be seen that all the frames have similar initial stiffness, while the BRB has the slightly higher initial stiffness. This observation is consistent with the fundamental periods of each system reported earlier. The steel frame with SVDs has the highest post-yielding stiffness and strength due to the post-transformation hardening behavior of SMAs.

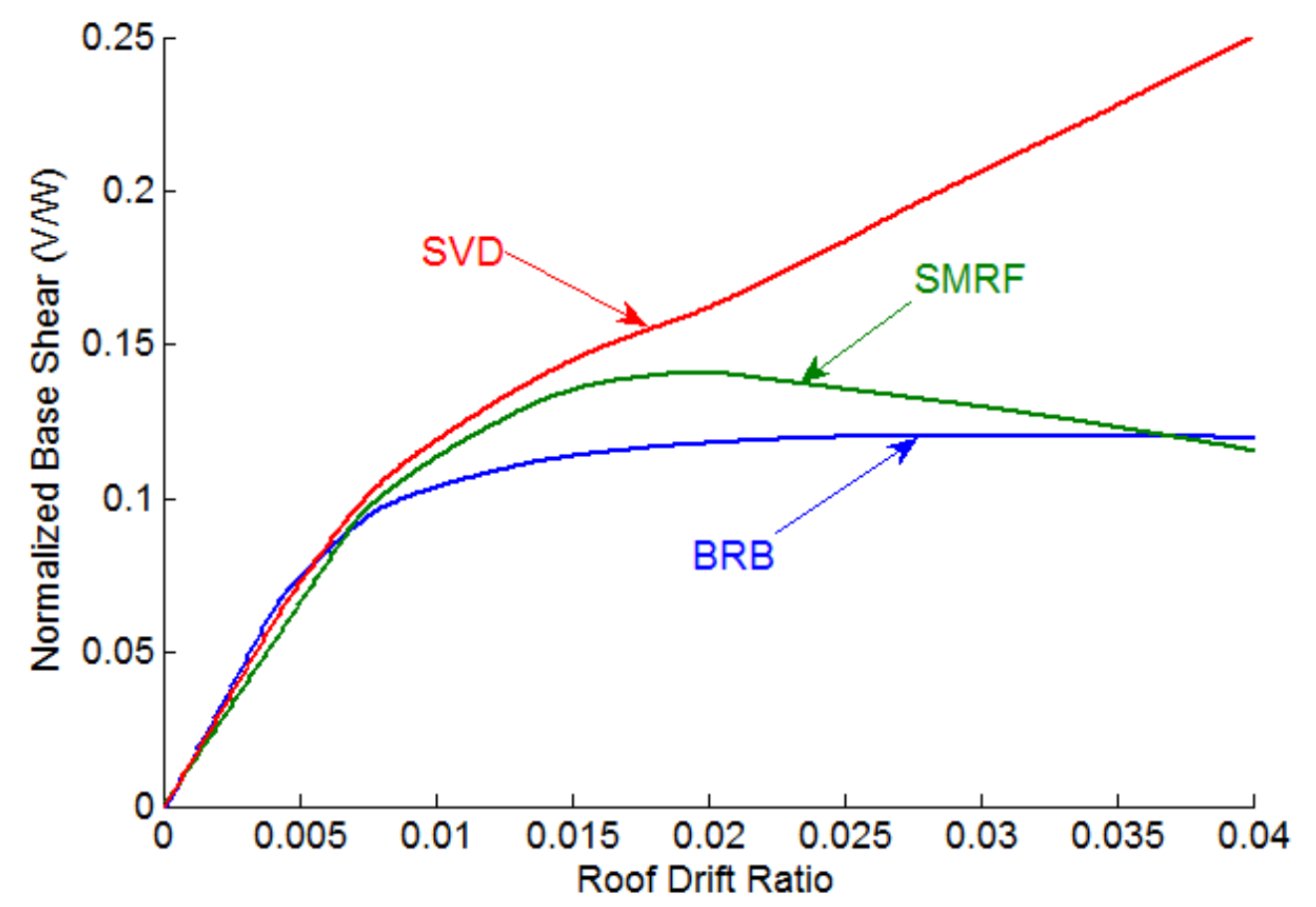

Figure 5-6. Pushover curves for three different steel frame systems

\subsubsection{Incremental Dynamic Analysis}

Incremental dynamic analysis (IDA) is employed to assess the seismic resistance of steel frame structures up to collapse using the 44 far field ground motions described above. For the IDA analysis, a series of nonlinear time history analyses is conducted under each of 44 ground motion records scaled to increasing intensity levels until the model becomes globally unstable (Vamvatsikos and Cornell, 2002). In this study, 5\% damped spectral acceleration at the fundamental period of the structure, $\mathrm{S} a\left(\mathrm{~T}_{l}\right)$, is used as the intensity measure, whereas the maximum inter-story drift ratio, peak absolute floor acceleration, and peak residual drift ratio are selected as engineering demand parameters. 
A potential collapse limit state corresponding to $5 \%$ inter-story drift is selected as suggested by previous studies considering the limitations in numerical simulation and insufficient resistance of the columns beyond this drift limit (Hsiao et al., 2013). Figure 5-7 displays interstory drift IDA curves for SMRF, SVD frame, and BRB frame, where a data point in each curve indicates the peak inter-story drift ratio of the frame under a ground motion record scaled at a specific spectral acceleration. The figure provides the traces for each of 44 ground motions. It can be seen that the record-to-record variability in peak drift among records is fairly large for each of three different steel frames. For SMRF and BRB frame, the slope of IDA curves decreases more rapidly as compared to that of SVD frame. For some ground motions, the curve flattens out at certain spectral acceleration, indicating the loss of lateral resistance of the structure.
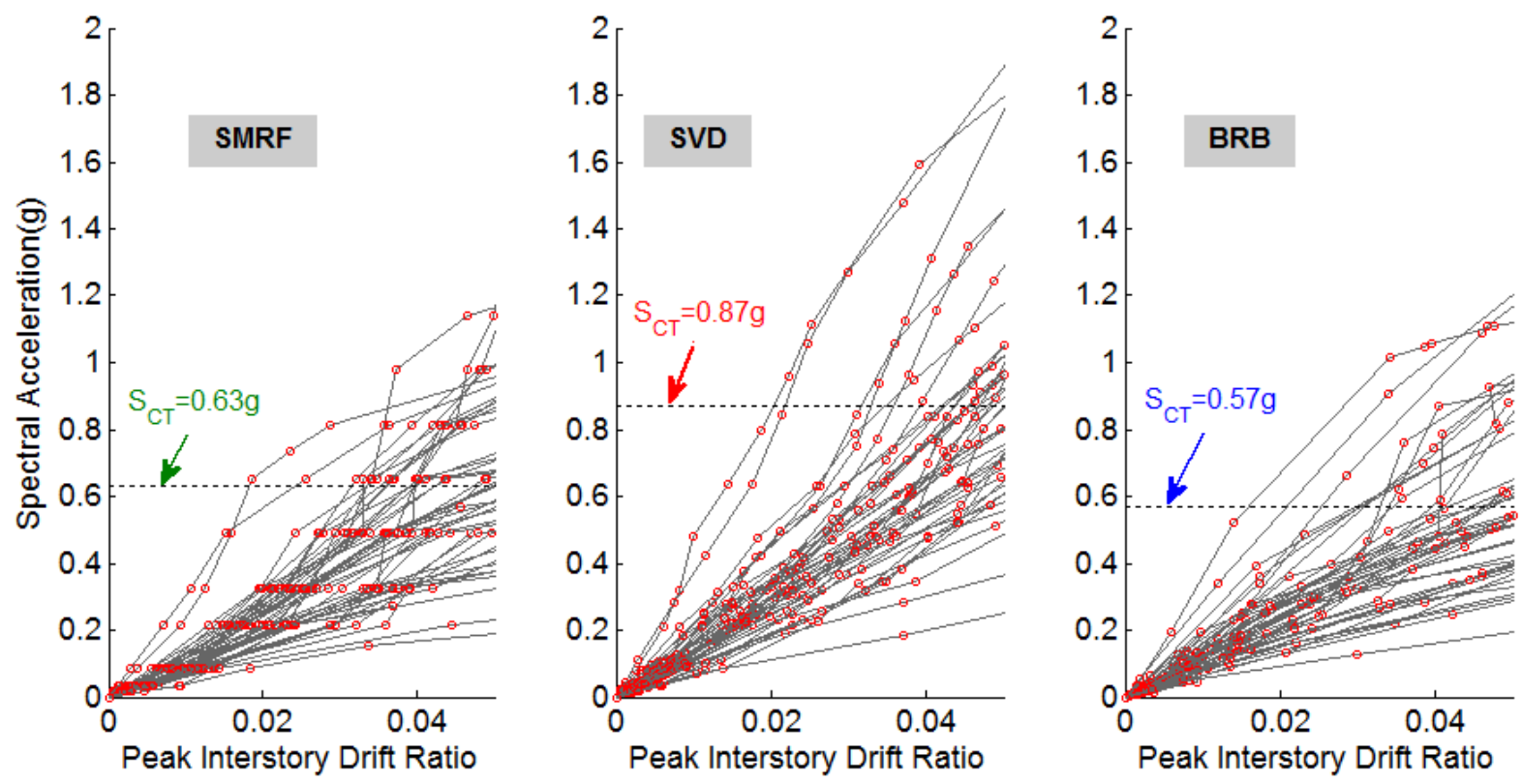

Figure 5-7. Maximum inter-story drift ratio IDA curves for: (a) SMRF, (b) SVD frame, and (c) BRB frame

FEMA 695 (FEMA, 2009) defines safety against collapse in terms of a collapse margin ratio (CMR). The CMR is defined as follows: 


$$
C M R=\frac{\hat{S}_{C T}}{S_{M T}}
$$

where $S_{C T}$ is the median collapse capacity defined as the ground motion intensity where half of the ground motions in the record set cause collapse of a structural model; and $\mathrm{S}_{\mathrm{Mт}}$ is the 5\%-damped spectral acceleration of the MCE ground motions at the fundamental period of the building. Factors such as ground motions variability (aleatory uncertainty) and uncertainty in design, analysis, and structural characteristics (epistemic uncertainty) affect the collapse of a seismic force-resisting system. Here, only aleatory uncertainty is considered to calculate the CMR for the three selected steel buildings and the results are summarized in Table 5-3. It can be seen that the frame installed with SVD provides higher median collapse capacity $\left(S_{C T}=0.87 \mathrm{~g}\right)$ and collapse margin ratio (CMR =2.49) compared to SMRF and BRB systems.

Table 5-3 Collapse safety parameters for different frames

\begin{tabular}{llll}
\hline Frame & $\hat{S}_{C T}(\mathrm{~g})$ & $S_{M T}(g)$ & $C M R$ \\
\hline SMRF & 0.63 & 0.33 & 1.91 \\
SVD & 0.87 & 0.35 & 2.49 \\
BRB & 0.57 & 0.37 & 1.51 \\
\hline
\end{tabular}

Incremental dynamic analysis curves for peak absolute floor acceleration and peak residual drift ratio are also plotted in Figures 5-8 and 5-9. Maximum floor acceleration is correlated with damage in non-structural components, while the residual drift ratio indicates post-event functionality of a structure. Residual story drifts are calculated by continuing the analyses for at least 20 seconds after the end of the seismic event. It can be seen from Figure 5-8 that BRB frame typically lead to peak floor accelerations that are lower than the other two frame systems for the ground motions scaled to different levels. Although for the SVD frame the ground motions are scaled to higher spectral levels for most ground motion records, the peak floor accelerations for the SVD frame is comparable with those for the SMRF system. 

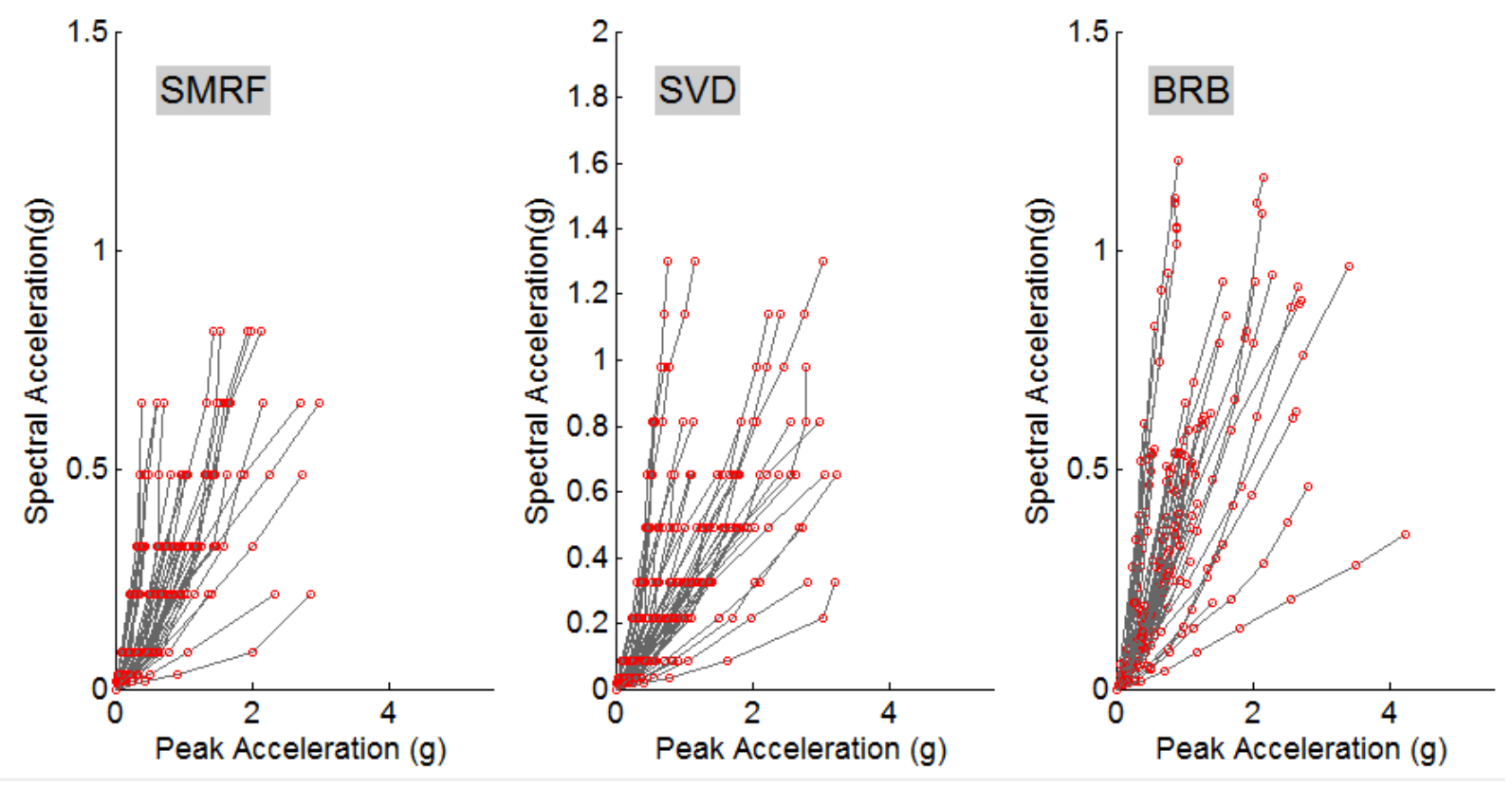

Figure 5-8. Maximum floor acceleration IDA curves for: (a) SMRF, (b) SVD frame, and (c) BRB frame

The results shown in Figure 5-9 reveal that the design of the building with SVDs significantly reduces the residual drift ratio at various levels of ground motions. Note that FEMA P-58 defines a damage state that requires realignment of structural frame and repairs for a residual story drift ratio of $0.5 \%$ and a damage state that requires major structural realignment that may not be economically and practically feasible for a residual story drift ratio of $1 \%$ (FEMA, 2012). It is found that the residual drifts for the SVD frame are very small for low and moderate intensity levels and below $1 \%$ even at high intensity levels for most of the ground motions. Only a few ground motion records produce residual drift ratios greater than $1 \%$ for the SVD frame at collapse level ground motion records. On the other hand, large residual drifts are observed for both SMRF and BRB frame at different ground motion intensity levels. 


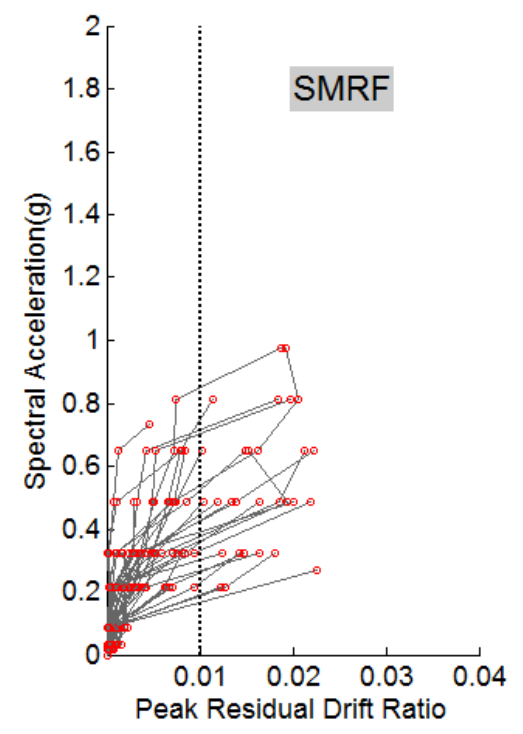

(a)

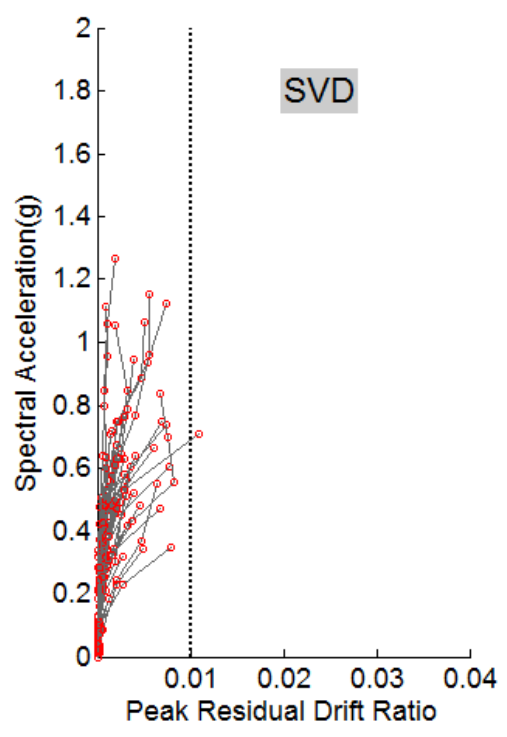

(b)

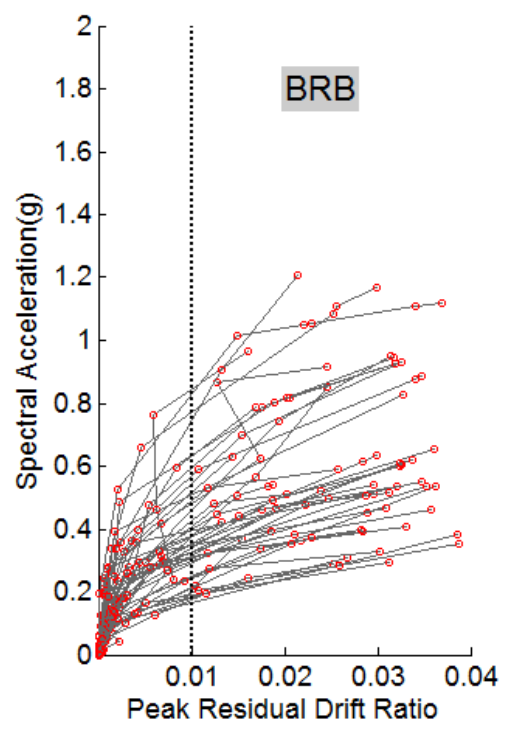

(c)

Figure 5-9. Maximum residual drift ratio IDA curves for: (a) SMRF, (b) SVD frame, and (c) BRB frame

In addition to IDA curves, the distribution of the peak inter-story drift ratio, peak floor acceleration ratio, and residual story drift ratio over the height of the building are computed for three lateral systems. Figure 5-10 provides the response of each frame to individual ground motion records as well as the median response for each floor level at $\mathrm{Sa}=0.33 \mathrm{~g}$, which corresponds to spectral acceleration at the fundamental period of SMRF for the MCE seismic hazard. To facilitate performance assessment of SMRF, SVD and BRB systems comparatively, the envelopes for the median of peak response quantities under 44 ground motion records for each system are also provided in Figure 5-11. It can be seen that each frame satisfactorily limits the peak inter-story drift response under various MCE level ground motions. Excessive drifts (above 10\%) are observed under two ground motion records for the SMRF frame. The SMRF frame typically has larger record-to-record variability in structural response, while the use of SVDs reduces the scatter in response. The BRB frame experiences the largest median inter-story drift ratio of $2.64 \%$ under 
44 ground motion records. The peak median responses are $2.29 \%$ and $2.14 \%$ for the SMRF and SVD frame, respectively.
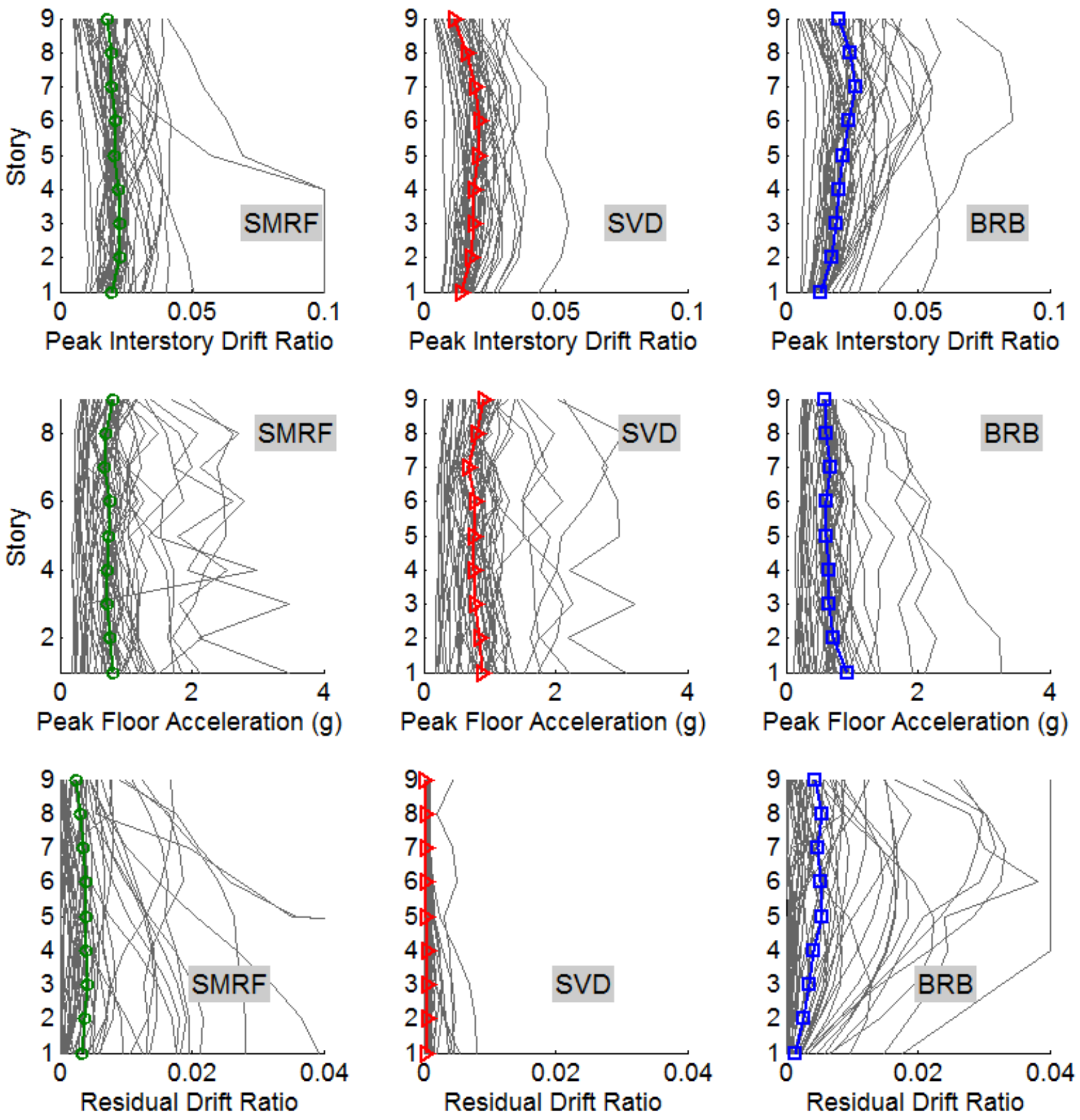

Figure 5-10. Profiles of (a) peak inter-story drift ratio, (b) peak floor acceleration, and (c) residual drift ratio at $\mathrm{MCE}$ level for each frame under individual ground motion records 

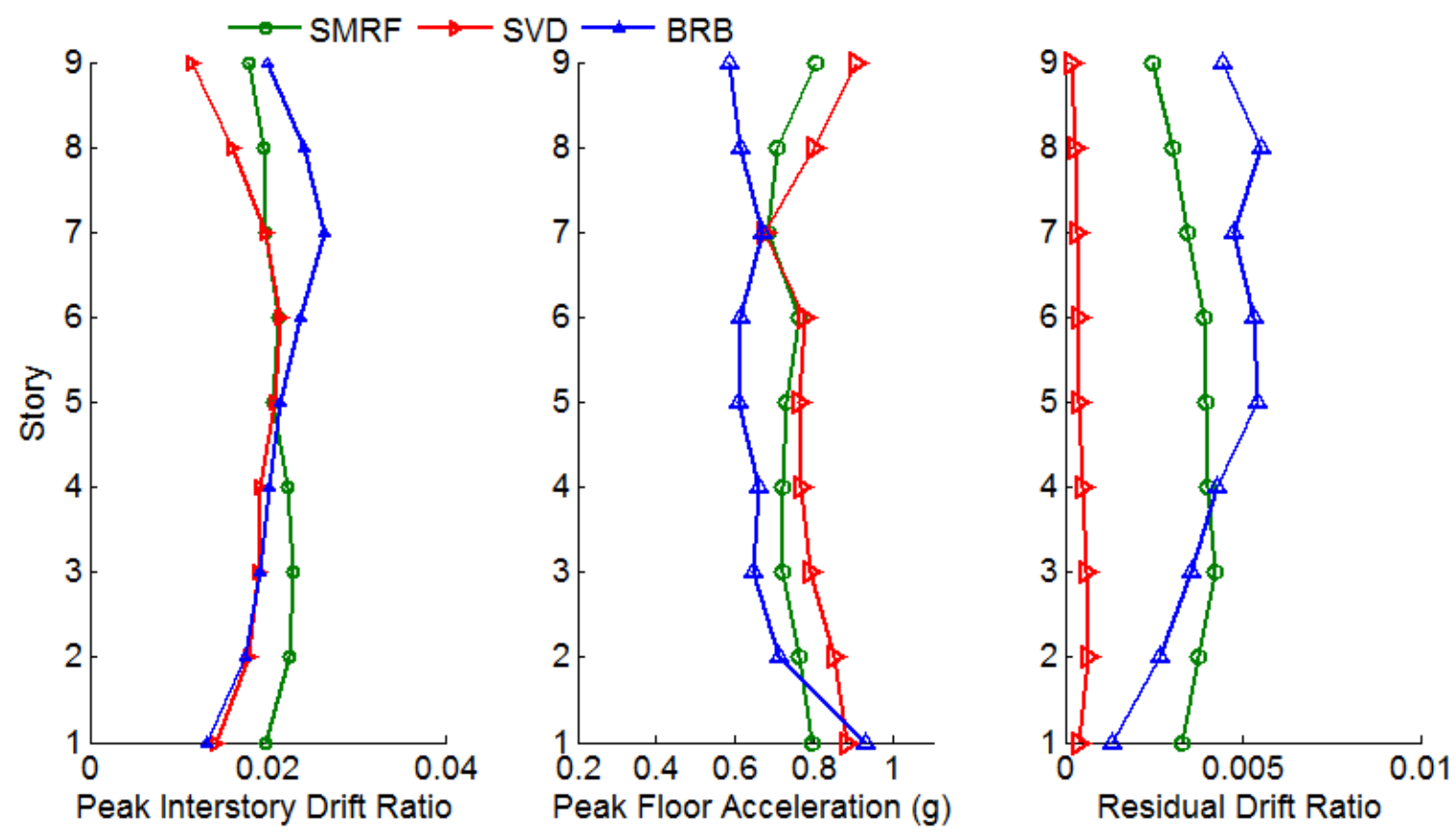

Figure 5-11. Envelopes for median of peak response quantities for SMRF, SVD, and BRB frames at MCE level

Although maximum values of the peak floor absolute acceleration are similar for each frame (0.93 $\mathrm{g}$ for BRB frame, $0.80 \mathrm{~g}$ for SMRF and 0.91 $\mathrm{g}$ SVD frame), the SMRF frame has the lowest peak acceleration values at each floor level except the first floor, while the SVD system produces larger accelerations at most of the floor levels as compared to the SVD frame. The SVD frame also has minimal residual drifts over the height of the 9-story frame, whereas especially the $\mathrm{BRB}$ system has considerable residual deformations. None of the ground motions scaled to $\mathrm{Sa}=$ 0.33 g produces peak residual drift ratios over $1 \%$ for the SVD frame. Also, the peak residual drift ratio is found to be above $0.5 \%$ only for one case out of 44 ground motion cases. On the other hand, the peak residual drift ratio over $1 \%$ is observed for the $32 \%$ and $77 \%$ of the ground motions records for the SMRF and BRB frame, respectively.

Finally, the peak median response quantities of each frame at different record set intensity are compared in Figure 5-12. In particular, the individual ground motions are scaled to following spectral intensities: $\mathrm{Sa}=0.22 \mathrm{~g}, \mathrm{Sa}=0.33 \mathrm{~g}, \mathrm{Sa}=0.49 \mathrm{~g}$, and $\mathrm{Sa}=0.65 \mathrm{~g}$. These spectral levels correspond to spectral acceleration at the fundamental period of the SMRF for the DBE, MCE, 1.5 
$\times$ MCE, and $2 \times$ MCE seismic hazard levels. It can be seen that the SVD frame has the lowest median peak inter-story drift at each spectral intensity level. The SMRF experiences the highest acceleration response at low (DBE) and very high $(2 \times \mathrm{MCE})$ seismic hazard levels. The SVD frame produces the slightly larger accelerations for the MCE and 1.5 × MCE levels (only 9\% and $7 \%$ higher, respectively) compared to the SMRF. The BRB frame has the smallest median peak acceleration values for all intensity levels. The results are in agreement with prior studies that found elasto-plastic systems generally produce smaller accelerations than flag-shaped hysteretic systems (Gavridou et al, 2014; Christopoulos, 2002). The results also highlight the excellent recentering ability of SVDs as the peak residual drifts are below $0.5 \%$ for the SVD frame for all seismic hazard levels. The BRB frame consistently possesses the highest residual drifts, while the SMRF frame experiences large residual drifts especially at high spectral intensity levels.
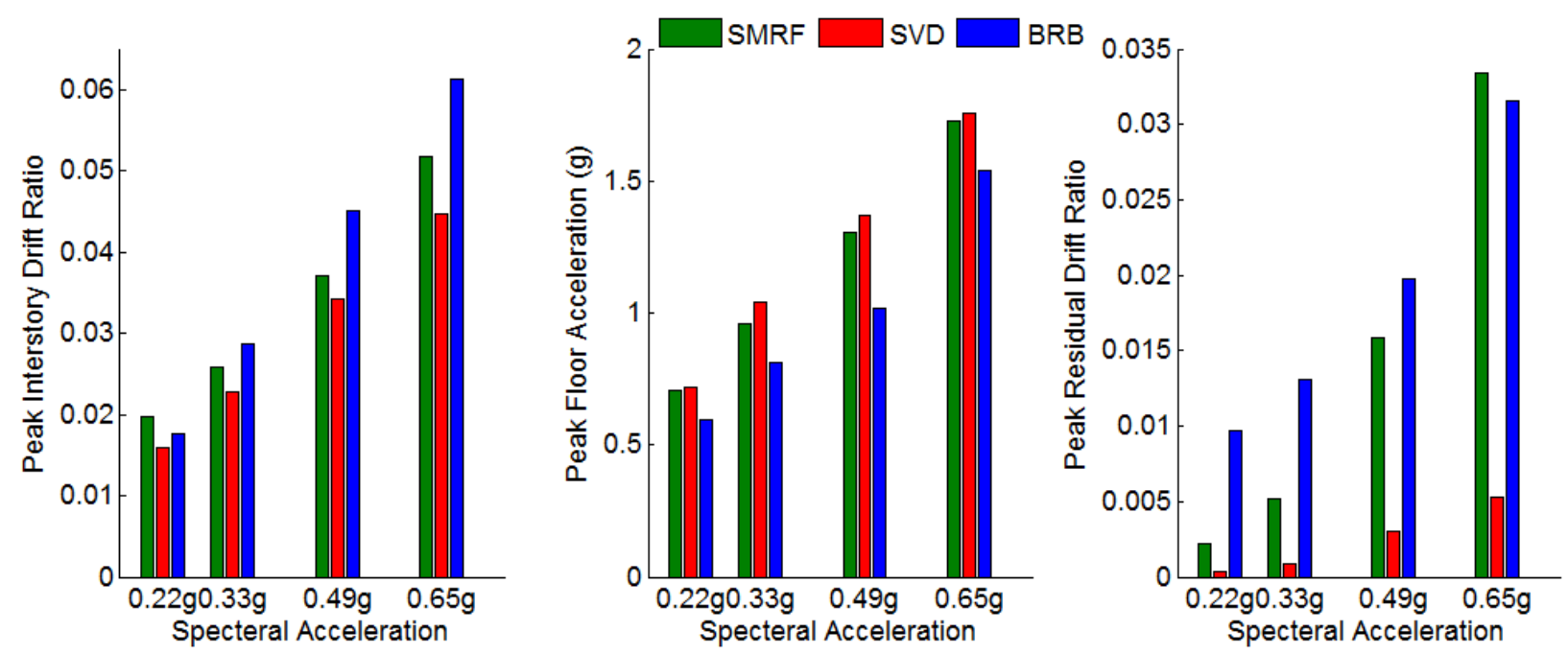

Figure 5-12. Median peak response quantities under 44 ground motion records scaled to different intensity levels for SMRF, SVD, and BRB frames

\subsubsection{Influence of SVD parameters}

In order to study the influence of SVD design parameters on the collapse and overall performance of the steel buildings designed with SVDs, a sensitivity analysis is also conducted. In particular, the original SVD designed discussed above (named as SVD-8) is altered such that in 
one design case more SMA elements are included to the device while the shear area of HD butyl damper is decreased (named as SVD-12), while in the other design case the size of the elastomeric component is increased while the number of SMA elements is reduced (named as SVD-4). In all designs, the force capacity of the SVDs at design displacement of $100 \mathrm{~mm}$ is kept to be the same, i.e. $300 \mathrm{kN}$. Table 5-4 summarizes the design parameters and modeling parameters for each SVD design case. In the table, $N_{S M A}$ shows the number of SMA cables, while $d_{S M A}$ represents the diameter of each SMA cable.

Table 5-4 SVD design and modeling parameters

\begin{tabular}{|c|c|c|c|c|c|c|c|c|c|c|c|}
\hline \multirow[t]{2}{*}{ Case } & \multicolumn{3}{|c|}{ SVD design parameters } & \multicolumn{5}{|c|}{ SMA model parameters } & \multicolumn{3}{|c|}{$\begin{array}{l}\text { HD butyl model } \\
\text { parameters }\end{array}$} \\
\hline & $N_{S M A}$ & $d_{S M A}$ & $\begin{array}{l}\text { Dimensions } \\
\text { of HD butyl }\end{array}$ & $\begin{array}{c}k_{1} \\
(\mathrm{kN} / \mathrm{mm})\end{array}$ & $\begin{array}{c}k_{2} \\
(\mathrm{kN} / \mathrm{mm})\end{array}$ & $\gamma$ & $\begin{array}{c}F_{a} \\
(\mathrm{kN})\end{array}$ & $\beta$ & $\begin{array}{c}K \\
(\mathrm{kN} / \mathrm{mm})\end{array}$ & $\begin{array}{c}C \\
(\mathrm{kN}- \\
\mathrm{s} / \mathrm{mm})\end{array}$ & $\alpha$ \\
\hline SVD-4 & 4 & 8 & $482 \times 482 \times 50$ & 1.458 & 0.497 & 1 & 26.52 & 1 & 2.02 & 1.41 & 0.8 \\
\hline SVD-8 & 8 & 8 & $406 \times 406 \times 50$ & 2.915 & 0.994 & 1 & 53.05 & 1 & 1.43 & 1.00 & 0.8 \\
\hline SVD-12 & 12 & 8 & $305 \times 305 \times 50$ & 4.373 & 1.491 & 1 & 79.57 & 1 & 0.81 & 0.56 & 0.8 \\
\hline
\end{tabular}

Table 5-5 Collapse safety parameters for frames with different SVD designs

\begin{tabular}{llll}
\hline Frame & $\hat{S}_{C T}(\mathrm{~g})$ & $S_{M T}(g)$ & $C M R$ \\
\hline SVD-4 & 0.79 & 0.34 & 2.32 \\
SVD-8 & 0.87 & 0.35 & 2.49 \\
SVD-12 & 0.74 & 0.37 & 2.00 \\
\hline
\end{tabular}

First, IDA analyses are conducted and the median collapse capacity and collapse margin ratio are calculated for the new SVD design cases. As can be seen from Table 5-5, the median collapse capacity does not change considerably with different SVD design parameters. Each SVD design case has higher $\mathrm{S}_{\mathrm{ct}}$ than the $\mathrm{SMRF}$ or BRB frames. However, CMR increased from 2.18 to 2.52 when larger elastomer is used and decrease to 1.98 when more SMA cables are used. With 
increasing number of SMA cables (i.e. from design case SVD-4 to SVD-12), the period of the

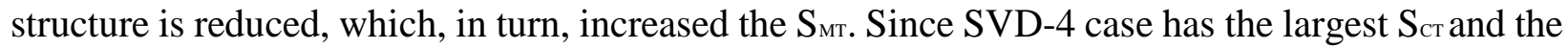
lowest $\mathrm{S}_{\mathrm{MT}}$, it attains the largest CMR value.

Then, the median peak response quantities for each SVD design case under 44 ground motion records for the $\mathrm{DBE}, \mathrm{MCE}, 1.5 \times \mathrm{MCE}$, and $2 \times \mathrm{MCE}$ seismic hazard levels are computed and shown in Figure5-13. It can be seen that the peak inter-story drift is not affected considerably at all hazard levels when different design parameters are used for the SVD. Nevertheless, there is a slight increase in the peak inter-story drift with the increasing number of SMA cables used in the device, especially at the $2 \times$ MCE seismic hazard level (8\% increase from SVD-4 to SVD- 12). This can be attributed to the reduction in viscous damping as smaller elastomer components are used in SVD-12 case compared to SVD-4 case. In addition, due to higher forces developed in SMA cables at large seismic intensities, the peak floor accelerations also increase with the increasing number of SMA cables used in the device. For the SVD-4 design case at $2 \times$ MCE seismic hazard level, the median peak acceleration is found to be $1.60 \mathrm{~g}$, while it increases to $1.75 \mathrm{~g}$ and $1.92 \mathrm{~g}$ for the SVD-8 and SVD-12, recording 9\% and 20\% increases, respectively.
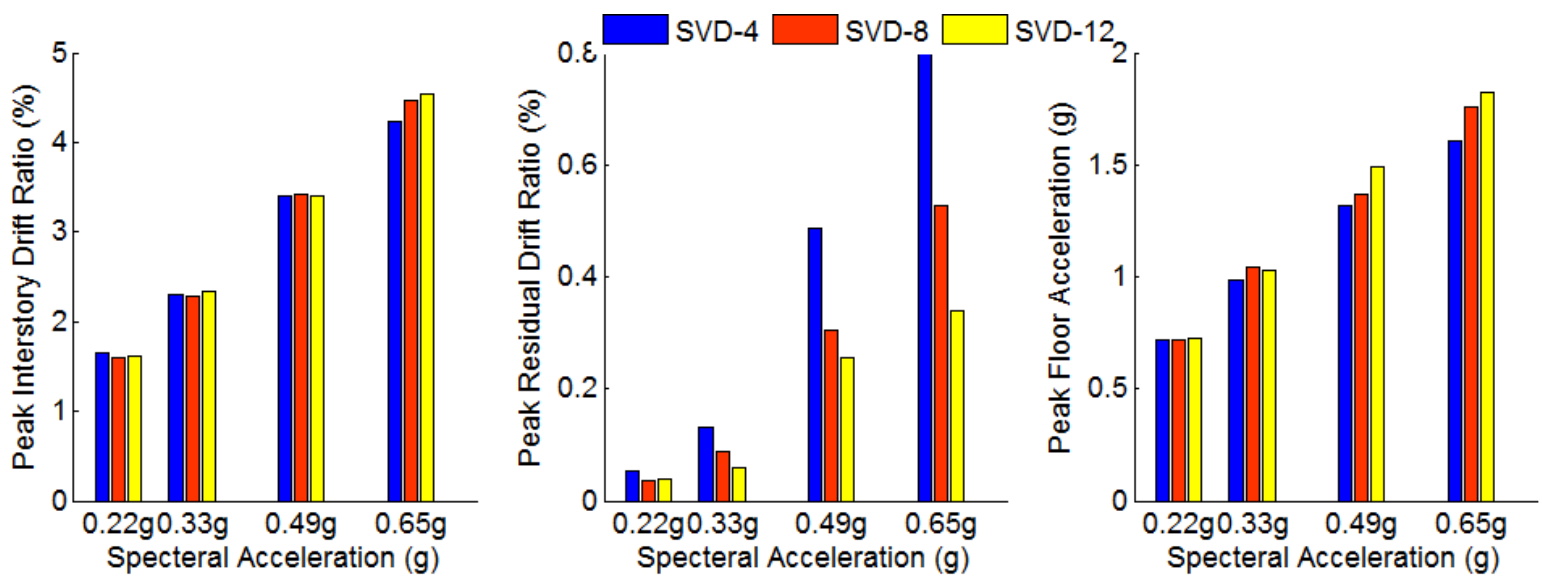

Figure 5-13. Median peak response quantities under 44 ground motion records scaled to different intensity levels for different designs of SVD frame

On the other hand, peak residual drift ratio has changed considerably almost at all hazard levels with the different SVD design parameters. At MCE hazard level, there are 40\% and 47\% reductions in peak residual drift response when the number of SMA cable at the device increases 
from 4 to 8 and 12, respectively. Similar reductions (38\% and 41\%) are observed at $1.5 \times \mathrm{MCE}$ seismic hazard level with the increasing number of SMAs in the SVD design. The highest decrease in the peak residual drift ratio is observed at $2 \times \mathrm{MCE}$ seismic hazard level, where it decreases from $0.72 \%$ for SVD-4 to $0.43 \%$ and $0.33 \%$ for SVD-8 and SVD- 12 , indicating $40 \%$ and $54 \%$ decreases, respectively. Note that for both SVD-8 and SVD-12 design cases, the peak residual drift ratios are below the critical $0.5 \%$ drift ratio, while it is somewhat higher for SVD-4 case. Nevertheless, for all SVD design cases residual drift ratios are considerably lower compared to SMRF and BRB frames, which have about $3 \%$ residual drift ratio at $2 \times$ MCE seismic hazard level. These results suggest that the SVD-8 design case provides both good collapse resistance and control of peak response quantities and can be considered as preferred design of the hybrid device.

\subsection{Closure}

In this section, a comparative seismic collapse assessment of a nine-story steel frame structure designed (i) as a special moment resisting system, (ii) with buckling restrained braces, and (iii) with superelastic viscous devices is conducted. Incremental dynamic analysis is employed to explore the behavior of the nine-story steel building with three different design configurations. A total of 44 ground motions records are used in nonlinear response history analyses. The performance of each system in mitigating the response of steel frame buildings under various levels of seismic hazard is assessed. A sensitivity analysis is also conducted to evaluate the effect of SVD design parameters on the collapse and overall performance of steel frames designed with SVDs.

Results show that the steel frame designed with SVDs has larger median collapse capacity compared to the conventional special moment resisting frame or steel frame with BRBs. The SVD frame effectively reduces the peak inter-story drift ratio at different seismic hazard levels without a considerable increase in the acceleration response. The SVD frame also produces minimal residual drifts even at very high seismic hazard levels. The significantly lower residual drifts observed in the SVD frame indicates that the repair costs of the steel frame buildings with SVDs will be less than that of the conventional SMRF and steel frames with BRBs after a seismic event. The results of the sensitivity analysis indicate that using larger viscoelastic component but fewer SMA cables in the design of SVD device improves the collapse resistance. However, it 
considerably increases peak residual drifts at different seismic hazard levels. Using a more balanced ratio between SMA and viscoelastic components in the SVD design can provide a satisfactory seismic performance. Overall, the results show the potential of SVDs as a passive seismic control device in improving collapse resistance of steel frame buildings and eliminating the damage under various seismic hazard levels. 



\section{SEISMIC FRAGILITY ESTIMATES OF STEEL FRAME BUILDINGS WITH SVDS}

\subsection{Introduction}

This section presents the seismic performance evaluation of a nine-story steel frame building designed either as a conventional steel moment resisting frame or with SVDs in a probabilistic framework. A nine-story steel moment resisting frame building designed with and without SVDs as described in Section 5 is employed for numerical analyses. Nonlinear response history analyses are carried out under 44 ground motion records that are scaled to different seismic hazard levels. Probabilistic demand models are developed for the selected engineering demand parameters. The resulting seismic demand relationships are employed in the fragility curve development to assess the effectiveness of SVDs in improving the performance of steel buildings over a range of seismic hazards.

\subsection{Building Description and Modeling}

A nine-story steel frame building, originally developed for the SAC Steel Frame Project (FEMA, 2000a; FEMA, 2000b) is selected for the numerical studies here. The detail of the building description, modeling, and design of the building with and without SVDs are described in Section 5.2.1 and 5.2.2, respectively.

\subsection{Nonlinear Dynamic Analysis}

To evaluate the effectiveness of the proposed SVD, the developed OpenSees models are analyzed considering a set of 44 far-field ground motion described in Section 4.3. Figure 6-1(a) illustrates the acceleration spectra of individual ground motion and median response spectrum. For the dynamic analysis, the ground motion records are scaled at two seismic hazard levels defined in ASCE 7-10, namely DBE and MCE level, so that average spectral ordinate of the records matches the target spectrum for the corresponding seismic hazard level at the fundamental period of the SMRF. To better characterize the seismic hazard at the site, the ground motions are scaled at two additional hazard levels, with probabilities of exceedance of $20 \%$ and $50 \%$ in 50 years. 
Figure 6-1(b) shows the median spectrum of the selected ground motions scaled at different seismic hazard levels. Using the 44 earthquake records scaled at four different seismic hazard levels, dynamic nonlinear response history analyses are performed for the SMRF and SVD frames and the structural responses of both frames are obtained.

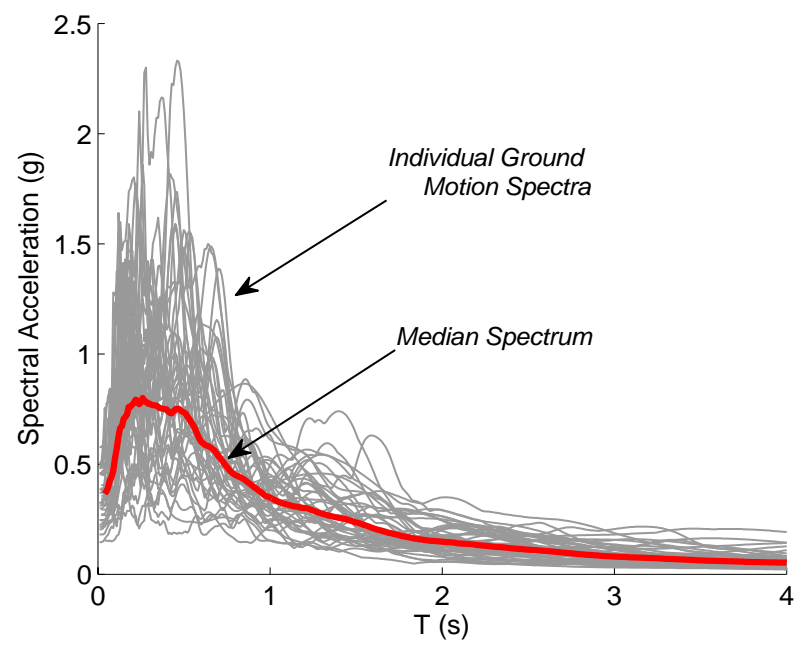

(a)

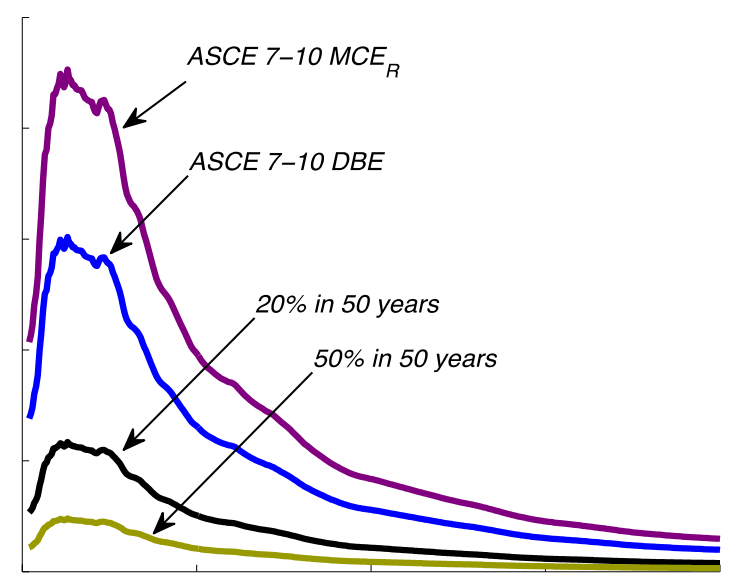

(b)

Figure 6-1 (a) Acceleration spectra of individual records and medium spectrum for 5\% damping, (b) median spectrum scaled to different hazard levels

Typical time histories of structural response under Northridge (recorded at Beverly Hills station) and Kobe (recorded at Shin-Osaka station) ground motion records scaled at MCE level are provided in Figure 6-2. Figure 6-2 shows the time histories of the inter-story drift ratio at the floor level where the maximum drift occurred for SMRF and SVD frames. It can be seen that peak interstory drift responses for both frames are similar for the Northridge and Kobe earthquakes. However, the SVD frame has significantly lower residual drift response. Figure 6-3 illustrates the time histories of the top floor absolute acceleration for both frames. Compared to Figure 6-3, the differences between the SMRF and SVD frames are much similar in terms of floor acceleration response. The peak acceleration response under Northridge earthquake is recorded to be $1.50 \mathrm{~g}$ and 1.62g for SMRF and SVD frames, respectively. The peak acceleration responses for SMRF and SVD frames under Kobe earthquake are $0.94 \mathrm{~g}$ and $0.99 \mathrm{~g}$, respectively. A probabilistic seismic 
performance evaluation of the structures using the dynamic response results is provided in the next section.
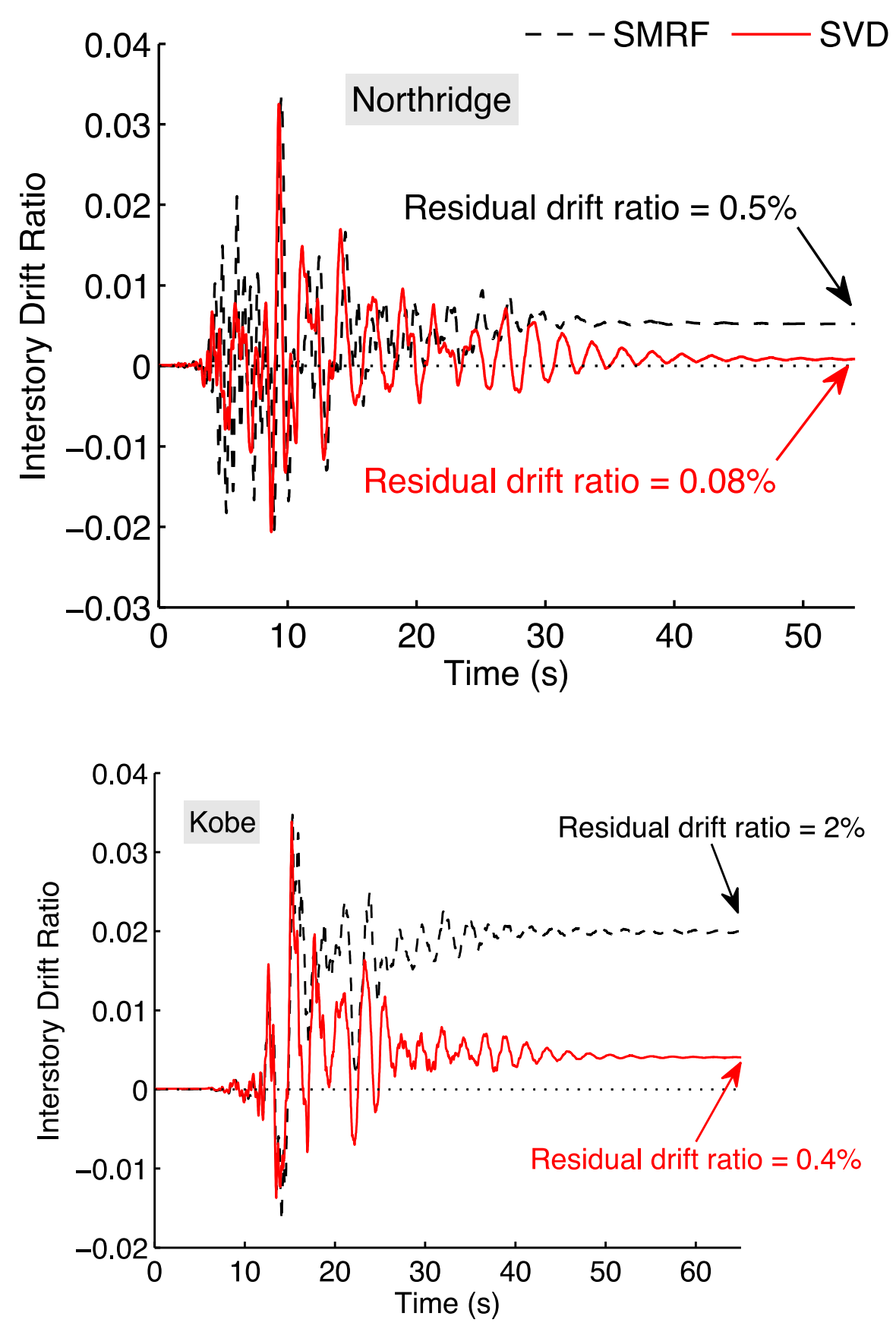

Figure 6-2 Time histories of inter-story drift for SMRF and SVD frames under Northridge and Kobe earthquakes. 

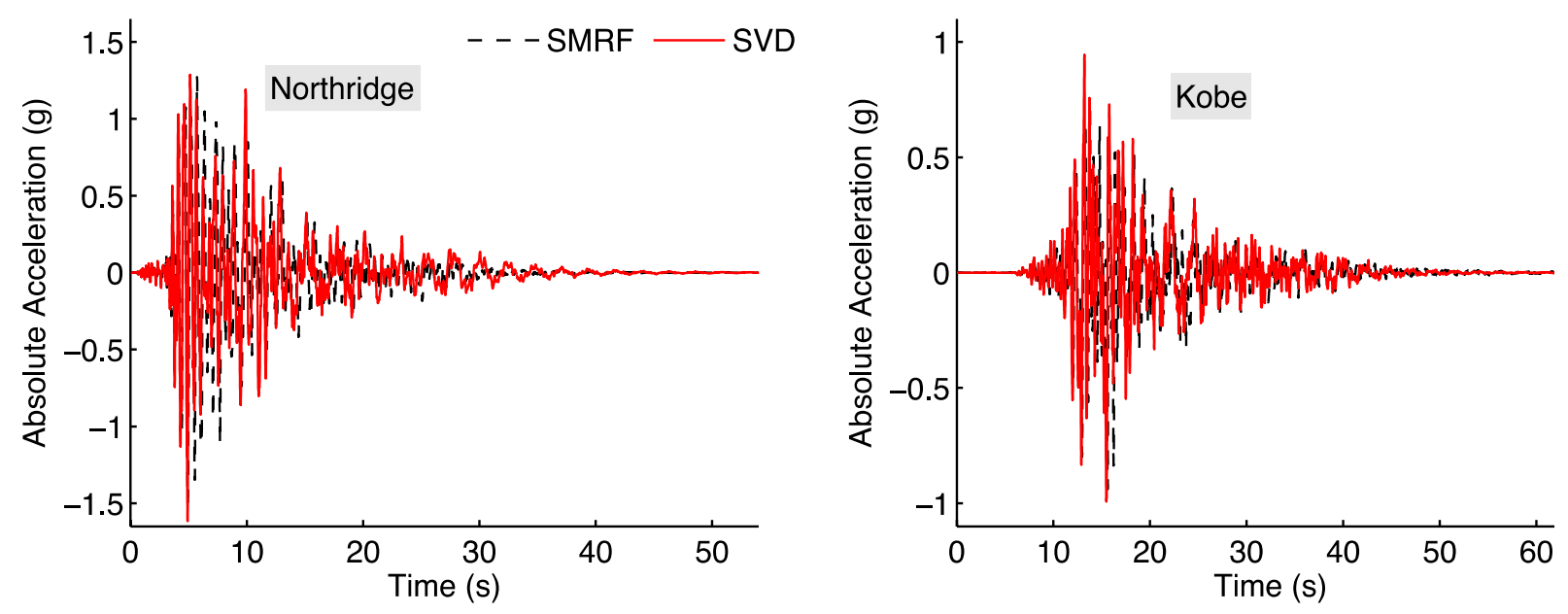

Figure 6-3 Time histories of top floor acceleration for SMRF and SVD frames under

Northridge and Kobe earthquakes.

\subsection{Probabilistic Performance Assessment}

\subsubsection{Seismic Fragilities}

Considering a broad scope of uncertainties associated with earthquake loading and seismic structural responses, seismic fragility is developed to quantitatively compare the seismic performances of the SMRF and SVD frames. Seismic fragility is defined as the conditional probability that an engineering demand parameter (EDP) of interest attains or exceeds a specified capacity level for given values of earthquake intensity measures (IMs). Typically, a specific capacity level corresponds to a specific structural/non-structural performance level. Therefore, seismic fragility can be used to describe the probability of exceeding a certain performance level.

Theoretically, seismic fragility curves can be developed empirically or analytically. Empirical fragility curves are built based on damage data from past earthquakes (Basoz and Kiremidjian, 1999; Shinozuka et al, 2000; Yamazaki et al., 1999). However, as the SVD frame is a newly proposed structural system, past damage data are unavailable. Many researchers have used analytical approaches (e.g., elastic spectral analysis (Hwang and Jernigan, 2000), nonlinear static analysis (Mander and Basoz, 2000; Shinozuka et al., 2000; Moschonas et al., 2009; Dutta and 
Mander, 1998), and nonlinear time-history analysis (Gardoni et al., 2003; Huang et al., 2010; Choi et al., 2004; Mackie and Stojadinović, 2003; Kafaeikivi et al., 2013; Dyanati et al., 2015) rather than empirical methods to derive fragility functions based on capacity and demand. In this study, nonlinear time-history analysis is conducted to assess seismic fragility. In particular, seismic fragility for the $k$ th failure mode is assessed using the following formulation:

$$
P_{f, k}(s)=P\left[C_{k}-D_{k}(\mathbf{x}, \boldsymbol{\Theta}) \leq 0 \mid s\right]
$$

where $s=$ seismic intensity measure (IM), and $C_{k}$ and $D_{k}$ are the capacity and EDP values for describing the $k$ th failure mode, respectively. For example, for peak inter-story drift $k=I D$ and for residual inter-story drift $k=R D$. While the capacities for the steel frame system are specified in the design code for various limit states, probabilistic EDP models are developed as described in the following subsection.

\subsubsection{Probabilistic Model Developments of Engineering Demand Parameters}

To measure structural damages in a building system, peak inter-story drift and residual inter-story drift, are two EDPs usually used (Badpay and Arbabi, 2008; Lin et al., 2010; Sabelli et al., 2003; Mayes et al., 2005; Wei, 2006; Uriz and Mahin, 2008; Ruiz-García and Miranda, 2010; Erochko et al., 2011; Song and Ellingwood, 1999). On the other hand, peak inter-story drift and peak floor acceleration are the EDPs used for the performance evaluation of drift-sensitive and acceleration sensitive non-structural components, respectively (Dyanati et al., 2016; Lin et al., 2010; Liu and Warn, 2012; Wanitkorkul and Filiatrault, 2008). Therefore, three probabilistic models are developed for three EDPs: peak inter-story drift, residual inter-story drift, and peak floor acceleration.

The EDP model formulation used in the study is written as:

$$
D_{k}\left(\mathbf{x}, \boldsymbol{\Theta}_{k}\right)=\theta_{k, 0}+\sum_{i=1}^{p_{k}} \theta_{k, i} h_{i}(\mathbf{x})+\sigma_{k} \varepsilon
$$

where $D_{k}\left(\mathbf{x}, \boldsymbol{\Theta}_{k}\right)=$ demand measure (or a suitable transformation such as natural logarithm); $h_{i}(\mathbf{x})$ $=$ explanatory functions (or a suitable transformation); $\boldsymbol{\theta}_{k}=\left(\theta_{k, 0}, \ldots, \theta_{k, p k}\right) ; \boldsymbol{\Theta}_{k}=\left(\boldsymbol{\theta}_{k}, \sigma_{k}\right)=$ a vector of unknown model parameters; $p_{k}=$ number of predictors; $\sigma_{k}=$ standard deviation of the model 
error; $\varepsilon=$ normal random variable with zero mean and unit variance; $\mathbf{x}=$ vector of basic variables (e.g., material properties, member dimensions, and imposed boundary conditions); and natural logarithmic transformations are used for all three demand quantities for variance stabilization. Since the characteristics of ground motions have high correlations with the seismic responses of the structures, seismic IMs are used to construct the potential explanatory functions $h_{i}(\mathbf{x})$. In this study, a total 12 of natural logarithms of the normalized IMs listed in Dyanati et al. (Dyanati et al. 2015) are used, which includes elastic pseudo spectral acceleration at the first mode period, PSA, and peak ground acceleration PGA.

To evaluate the model parameters, maximum likelihood approach is adopted. Since peak inter-story drift and residual inter-story drift both are used for structural performance evaluation, the joint distribution of these two quantities is desired. In this study, it is found that the numerical inter-story drift and residual drift responses obtained from the dynamic analysis in OpenSees are extremely large for some earthquakes, which is believed that the structure has collapsed. Therefore, those large value responses themselves are not meaningful. However, one can believe that the true responses for these earthquake cases should be larger than the collapse limit. In this study, such collapse limit $\left(\delta_{c}\right)$ for either the inter-story drift or residual drift is considered to be $10 \%$, then $10 \%$ is set as the lower bound value for those cases.

Following Gardoni et al. (Gardoni et al., 2003), with the considering the equality data and lower bound data, the likelihood function for the bi-variant, peak inter-story drift and residual inter-story drift, is used to assess $\boldsymbol{\Theta}_{I D}$ and $\boldsymbol{\Theta}_{R D}$, written as:

$$
L(\boldsymbol{\Theta})=\prod_{\text {equality data }} \varphi_{2}\left(\frac{\hat{D}_{I D, i}-D_{I D, i}}{\sigma_{I D}}, \frac{\hat{D}_{R D, i}-D_{R D, i}}{\sigma_{R D}}, \rho\right) \prod_{\text {lower bound data }} \Phi_{2}\left(-\frac{\hat{D}_{I D, i}-\delta_{c}}{\sigma_{I D}},-\frac{\hat{D}_{R D, i}-\delta_{c}}{\sigma_{R D}}, \rho\right)
$$

where $\varphi$ and $\Phi=$ bi-variate probability density function and cumulative density function of two standard normal random variables, respectively, $D_{k}=$ numerical response and that symbol represents the point estimation of demand quantity predicted by Eq. (6-2), and $\rho=$ correlation coefficient between peak inter-story and residual inter-story drifts. 
Meanwhile, for the cases with extreme value of drift responses, the peak floor acceleration responses can only be used as lower bound data as well. Unless the peak floor acceleration response is also extreme large, then a collapse limit (ac) is used as the lower bound value. In this study, ac $=3.5 \mathrm{~g}$ is adopted following Dyanati et al. (Dyanati et al., 2016). Thus, to assess $\boldsymbol{\Theta}_{P F A}$, the likelihood function can be written as follows:

$$
L(\boldsymbol{\Theta})=\prod_{\text {equality data }} \varphi\left(\frac{\hat{D}_{P F A, i}-D_{P F A, i}}{\sigma_{P F A}}\right) \prod_{\text {lower bound data }} \Phi\left(-\frac{\hat{D}_{P F A, i}-D_{P F A, i}}{\sigma_{P F A}}\right)
$$

With all the potential explanatory functions, the full model size is 12 . An all possible subset model selection method (Sheather, 2008) is adopted to select the explanatory function(s) that contribute to the demand prediction. Finally, it is found that the same demand formulations are obtained for both buildings with and without SVD. The demand models for the peak inter-story drift, residual inter-story drift, and peak floor acceleration are shown as following:

$$
\begin{gathered}
D_{I D}\left(\mathbf{x}, \boldsymbol{\Theta}_{I D}\right)=\theta_{I D 0}+\theta_{I D, 1} \cdot \log \left(\frac{\mathrm{PSA}}{g}\right)+\sigma_{I D} \varepsilon \\
D_{R D}\left(\mathbf{x}, \boldsymbol{\Theta}_{I D}\right)=\theta_{R D, 0}+\theta_{R D, 1} \cdot \log \left(\frac{\mathrm{PSA}}{g}\right)+\sigma_{R D} \varepsilon \\
D_{P F A}\left(\mathbf{x}, \boldsymbol{\Theta}_{P F A}\right)=\theta_{P F A, 0}+\theta_{P F A, 1} \cdot \log \left(\frac{\mathrm{PGA}}{g}\right)+\sigma_{P F A} \varepsilon
\end{gathered}
$$

Table 6-1 and Table 6-2 provide the statistics of the model parameters obtained from the maximum likelihood method. 
Table 6-1 Statistics of model parameters in the peak inter-story drift $(k=$ ID) and residual interstory drift $(k=R D)$ models.

\begin{tabular}{|c|c|c|c|c|c|c|c|c|c|c|}
\hline \multirow{2}{*}{$\begin{array}{c}\text { Frame } \\
\text { type }\end{array}$} & \multirow{2}{*}{$\begin{array}{l}\text { Para- } \\
\text { meter }\end{array}$} & \multirow{2}{*}{ Mean } & \multirow{2}{*}{ Std. } & \multicolumn{7}{|c|}{ Correlation coefficient } \\
\hline & & & & $\theta_{I D, 0}$ & $\theta_{I D, 1}$ & $\sigma_{I D}$ & $\theta_{R D, 0}$ & $\theta_{R D, 1}$ & $\sigma_{R D}$ & $P$ \\
\hline \multirow{7}{*}{ SMRF } & $\theta_{I D, 0}$ & 2.308 & 0.069 & 1.000 & 0.873 & 0.327 & 0.603 & 0.514 & 0.063 & 0.227 \\
\hline & $\theta_{I D, 1}$ & 0.963 & 0.030 & 0.873 & 1.000 & 0.326 & 0.525 & 0.600 & 0.135 & 0.206 \\
\hline & $\sigma_{I D}$ & 0.418 & 0.023 & 0.327 & 0.326 & 1.000 & 0.187 & 0.199 & 0.281 & 0.488 \\
\hline & $\theta_{R D, 0}$ & 1.622 & 0.217 & 0.603 & 0.525 & 0.187 & 1.000 & 0.865 & 0.249 & 0.159 \\
\hline & $\theta_{R D, 1}$ & 1.648 & 0.100 & 0.514 & 0.600 & 0.199 & 0.865 & 1.000 & 0.237 & 0.146 \\
\hline & $\sigma_{R D}$ & 1.424 & 0.082 & 0.063 & 0.135 & 0.281 & 0.249 & 0.237 & 1.000 & 0.358 \\
\hline & $\rho$ & 0.690 & 0.043 & 0.227 & 0.206 & 0.488 & 0.159 & 0.146 & 0.358 & 1.000 \\
\hline \multirow{7}{*}{ SVD } & $\theta_{I D, 0}$ & 1.810 & 0.065 & 1.000 & 0.863 & 0.033 & 0.529 & 0.463 & -0.023 & 0.065 \\
\hline & $\theta_{I D, 1}$ & 0.752 & 0.027 & 0.863 & 1.000 & 0.040 & 0.458 & 0.531 & 0.005 & 0.068 \\
\hline & $\sigma_{I D}$ & 0.456 & 0.025 & 0.033 & 0.040 & 1.000 & -0.032 & -0.013 & 0.366 & 0.450 \\
\hline & $\theta_{R D, 0}$ & -0.824 & 0.138 & 0.529 & 0.458 & -0.032 & 1.000 & 0.864 & 0.010 & -0.015 \\
\hline & $\theta_{R D, 1}$ & 1.076 & 0.058 & 0.463 & 0.531 & -0.013 & 0.864 & 1.000 & 0.031 & 0.016 \\
\hline & $\sigma_{R D}$ & 0.995 & 0.054 & -0.023 & 0.005 & 0.366 & 0.010 & 0.031 & 1.000 & 0.395 \\
\hline & $\rho$ & 0.601 & 0.050 & 0.065 & 0.068 & 0.450 & -0.015 & 0.016 & 0.395 & 1.000 \\
\hline
\end{tabular}

Table 6-2 Statistics of model parameters in the peak floor acceleration ( $k=$ PFA) models.

\begin{tabular}{ccccccc}
\hline \multirow{2}{*}{$\begin{array}{c}\text { Frame } \\
\text { type }\end{array}$} & Parameter & Mean & $\begin{array}{c}\text { Standard } \\
\text { deviation }\end{array}$ & \multicolumn{3}{c}{ Correlation coefficient } \\
\cline { 5 - 7 } & $\theta_{P F A, 0}$ & 0.152 & 0.034 & 1.000 & 0.4731 & -0.260 \\
SMRF & $\theta_{P F A, 1}$ & 0.657 & 0.023 & 0.4731 & 1.000 & -0.317 \\
& $\sigma_{P F A}$ & 0.370 & 0.020 & -0.260 & -0.317 & 1.000 \\
\hline \multirow{2}{*}{ SVD } & $\theta_{P F A, 0}$ & 0.173 & 0.037 & 1.000 & 0.726 & 0.000 \\
& $\theta_{P F A, 1}$ & 0.682 & 0.027 & 0.726 & 1.000 & -0.039 \\
& $\sigma_{P F A}$ & 0.340 & 0.018 & 0.000 & -0.039 & 1.000 \\
\hline
\end{tabular}




\subsubsection{Results}

Based on ASCE 41-06 (ASCE, 2007), three performance levels are considered in this study: immediate occupancy (IO), life safety (LS), and collapse prevention (CP). The capacities corresponding to these three performance levels are given in Table 6-3. In Table 6-3, the capacity values for the structural performance are adopted from ASCE 41-06, while the capacity values for the non-structural performance are adopted from HAZUS (FEMA, 2014) corresponding to generic acceleration-sensitive and drift-sensitive components. To account for the variability in the capacity, all the capacity is assumed to follow lognormal distribution with medium values shown in Table 6-3 and a coefficient of variation (COV) of $30 \%$ following Ellingwood and Wen (Ellingwood and Wen, 2005).

Table 6-3 Medium of inter-story drift, residual inter-story drift, and peak floor acceleration capacities for various performance levels.

\begin{tabular}{|c|c|c|c|c|}
\hline \multirow{2}{*}{$\begin{array}{l}\text { Performance } \\
\text { level }\end{array}$} & \multicolumn{2}{|c|}{ Structural } & \multicolumn{2}{|c|}{ Non-structural } \\
\hline & $\begin{array}{l}\text { Peak inter- } \\
\text { story drift }\end{array}$ & $\begin{array}{l}\text { Residual inter-story } \\
\text { drift }\end{array}$ & $\begin{array}{l}\text { Acceleration } \\
\text { sensitive }\end{array}$ & $\begin{array}{c}\text { Drift } \\
\text { sensitive }\end{array}$ \\
\hline IO & $0.7 \%$ & $0.05 \%$ & $0.7(\mathrm{~g})$ & $0.4 \%$ \\
\hline $\mathrm{LS}$ & $2.5 \%$ & $0.5 \%$ & $1.0(\mathrm{~g})$ & $0.8 \%$ \\
\hline $\mathrm{CP}$ & $5.0 \%$ & $2 \%$ & $2.2(\mathrm{~g})$ & $2.5 \%$ \\
\hline
\end{tabular}

With the demand models developed as shown in Eqs. (6-5), (6-6), and (6-7), and the capacity values shown in Table 6-3, seismic fragility of structural and non-structural performance for three performance levels can be obtained based on Eq. (6-1). Note that since the structural performance is determined when the perk inter-story drift exceeds its limit or when the residual inter-story drift exceeds its limit, the seismic fragility for structural performance should be assessed by 


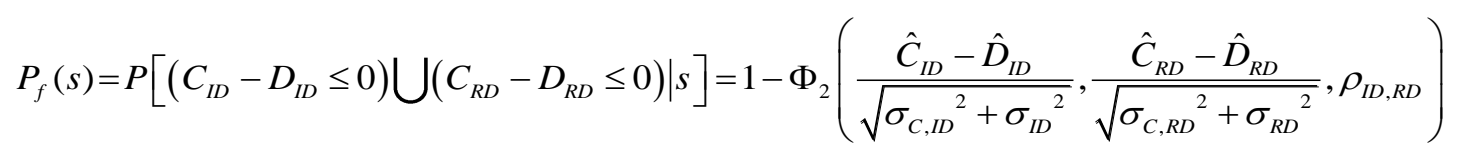

where $\hat{C}_{k}=$ mean of logarithm of capacity, $\rho_{I D, R D}=$ correlation between limit state $\left(C_{I D}-D_{I D} \leq 0\right)$ and limit state $\left(C_{R D}-D_{R D} \leq 0\right)$. Since the capacity and demand can be treated as statistically independent, following Huang et al. (Huang et al., 2010), $\rho_{I D, R D}$ can be calculated by

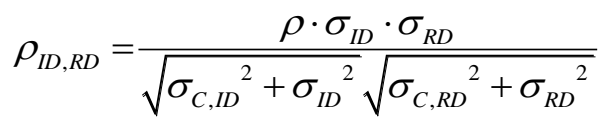

Since the performance for non-structural component is determined by a single limit state, the seismic fragility for non-structural component is determined by

$$
P_{f}(s)=1-\Phi\left(\frac{\hat{C}_{k}-\hat{D}_{k}}{\sqrt{\sigma_{C, k}^{2}+\sigma_{k}^{2}}}\right)
$$

where $k=I D$ when evaluating drift-sensitive nonstructural performance, and $k=R D$ when evaluating acceleration-sensitive nonstructural performance.

Fragility curves for three performance levels (IO, LS, and CP) are developed for structural, non-structural drift sensitive and non-structural acceleration sensitive performances, as shown in Figures 6-4, 6-5, and 6-6, respectively. In terms of structural and drift-sensitive nonstructural performance, Figures 6-4 and 6-5 show that the building design with SVDs has lower probability of "failure" (i.e. better seismic performance) than the SMRF building given a specific seismic IM, particularly for lower performance levels (CP level). However, for the high performance level (IO level), the structural performances of the SMRF and SVD frames are nearly the same. In terms of acceleration-sensitive nonstructural performance, Figure 6-4 indicates that the seismic performances of both building are about the same for all performance levels, while the SVD frame has slightly better for lower seismic intensities and the SMRF has marginally better for higher seismic intensities. Overall, the results shown in Figures 6-4, 6-5, and 6-6 reveal the steel buildings 
designed with SVDs can significantly improve structural and drift-sensitive non-structural performance, but will not impact the acceleration-sensitive non-structural performance. Since seismic fragilities are directly related to damage states, the results also indicate that the implementation of the proposed SVD can significantly lower the post-earthquake costs.

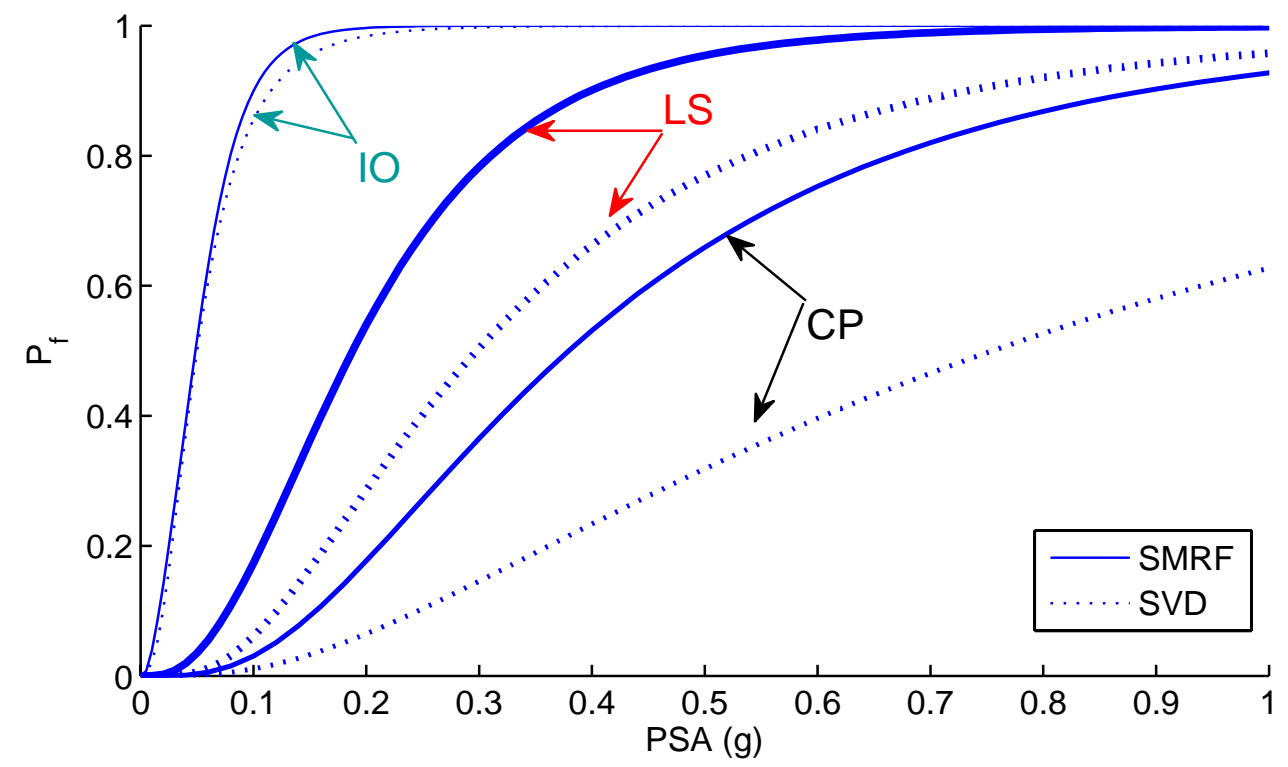

Figure 6-4.Fragility curves of structural components 


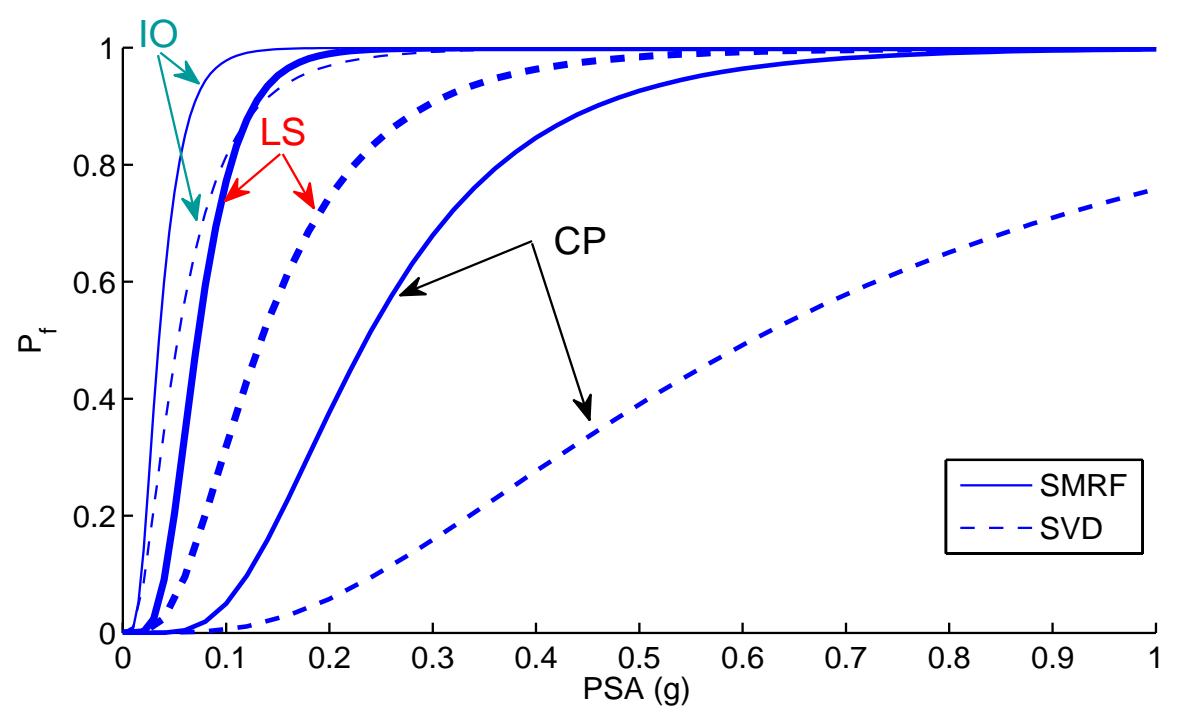

Figure 6-5 Fragility curves of nonstructural drift sensitive components

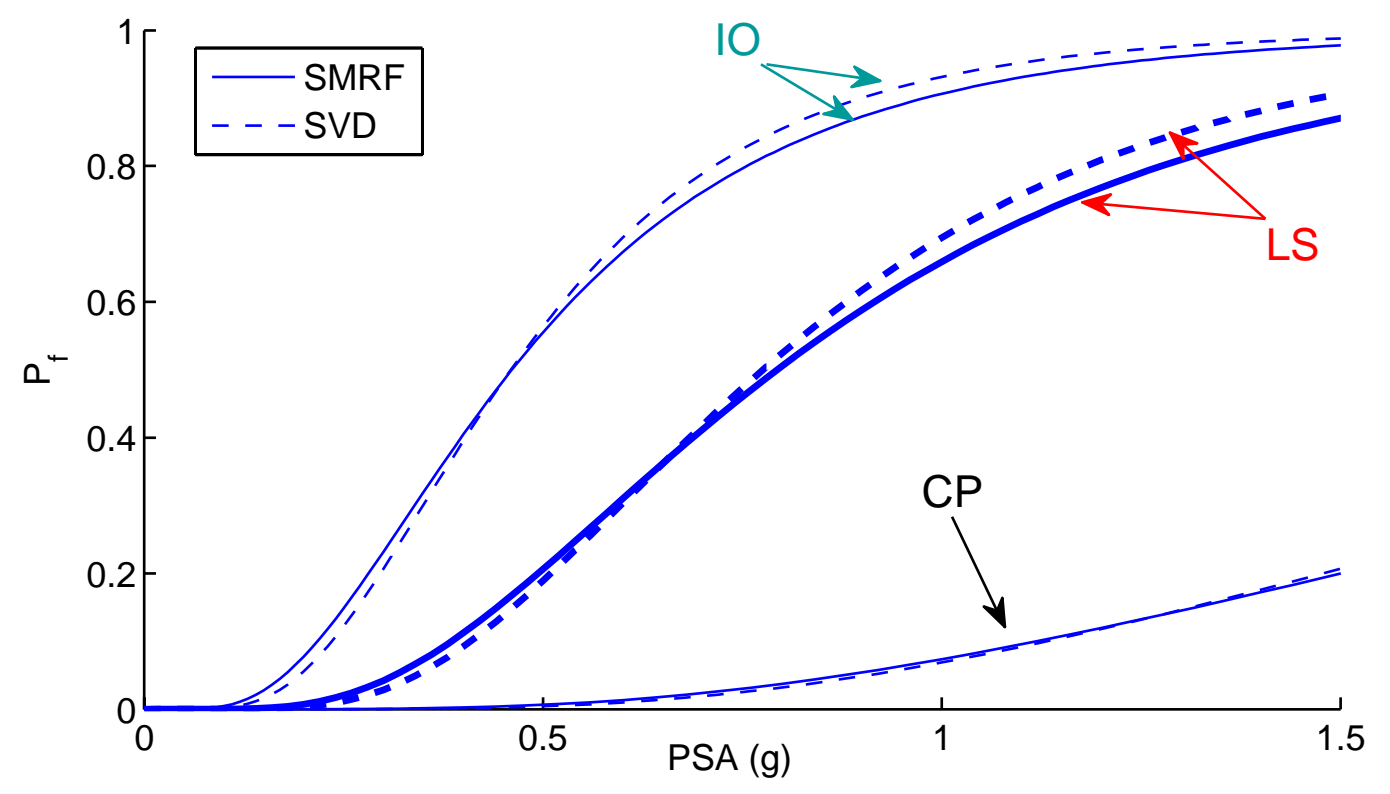

Figure 6-6 Fragility curves of nonstructural acceleration sensitive components 


\subsection{Closure}

The seismic performance of steel frame structures designed with and without a supplementary seismic control system is investigated in this Section using a probabilistic framework. A nine-story steel building is designed according to the current design guidelines either as a steel moment resisting frame (SMRF) or as a steel frame with installed SVDs (or called SVD frame). Structural responses of steel frame buildings under various seismic hazard levels are evaluated through nonlinear response history analyses.

Probabilistic demand models are then developed for inter-story drift, residual inter story drift and peak floor acceleration. With the developed demand models, the seismic fragility curves at different performance levels are created for both SMRF and SVD frames to assess the effectiveness of the SVDs. The results suggest that the steel frame buildings designed with supplemental SVD systems can considerably improve the structural and drift-sensitive nonstructural performances, especially at high seismic hazard levels, while provide almost the same performance for the acceleration-sensitive non-structural components with traditional SMRF systems. This suggests that the implementation of SVDs can potentially reduce the post-earthquake losses. 



\section{MAINSHOCK-AFTERSHOCK PERFORMANE EVALUATION OF STEEL FRAME BUILDINGS WITH SVDS}

\subsection{Introduction}

This section explores the aftershock collapse performance of steel buildings designed with superelastic viscous dampers under seismic sequences. A nine-story steel moment resisting frame building designed with and without SVDs and described in Section 5 is employed for numerical analyses. A mainshock incremental dynamic analysis (IDA) is conducted for the SMRF and SVD frames using a total of ten as-recorded seismic sequences. The specific levels of post-mainshock inter-story drift ratios are then induced in both frames and an aftershock IDA analysis is conducted for the mainshock-damaged buildings. The maximum inter-story drift and residual drift IDA curves are developed and compared for both frames at different mainshock damage levels. The results are analyzed in terms of the aftershock collapse capacity, collapse fragility, and collapse capacity at demolition. The effect of aftershock ground motion polarity on the performance of both frames is also explored.

\subsection{Effect of Aftershocks}

In traditional seismic design of structures, only one earthquake event called as mainshock is generally considered in the design process, while the effect of aftershocks is ignored. Aftershocks typically originate near the rupture zone of the mainshock and include a sequence of events with varying magnitudes and can occur hours, months or even years after the mainshock. For example, in Gorkha, Nepal, a local magnitude $\left(M_{L}\right) 7.6$ (a moment magnitude $M_{w}$ of 7.8) earthquake was followed by 120 earthquakes with $M_{L}$ greater than 4.0 within the first 12 hours. There were 42 earthquakes with $M_{L}$ greater than 5.0 within 30 days as shown in Figure 7-1(a). Among these aftershocks, four ground motions with a local magnitude larger than 6.0 were observed within 20 days and the largest aftershock with a $M_{L}=6.9\left(M_{w}=7.3\right)$ occurred on May 12, 2015 (NSC, 2015). Similarly, after $M_{w} 8.8$ February 27, 2010 Chile earthquake, there were 306 aftershocks with magnitudes equal to or greater than 5.0, among which 21 had magnitudes greater than 6.0, until April 26, 2010. Figure 7-1(b) shows aftershocks with $M_{w}$ greater than 5.0 within first two days of February 27 Chile earthquake. 


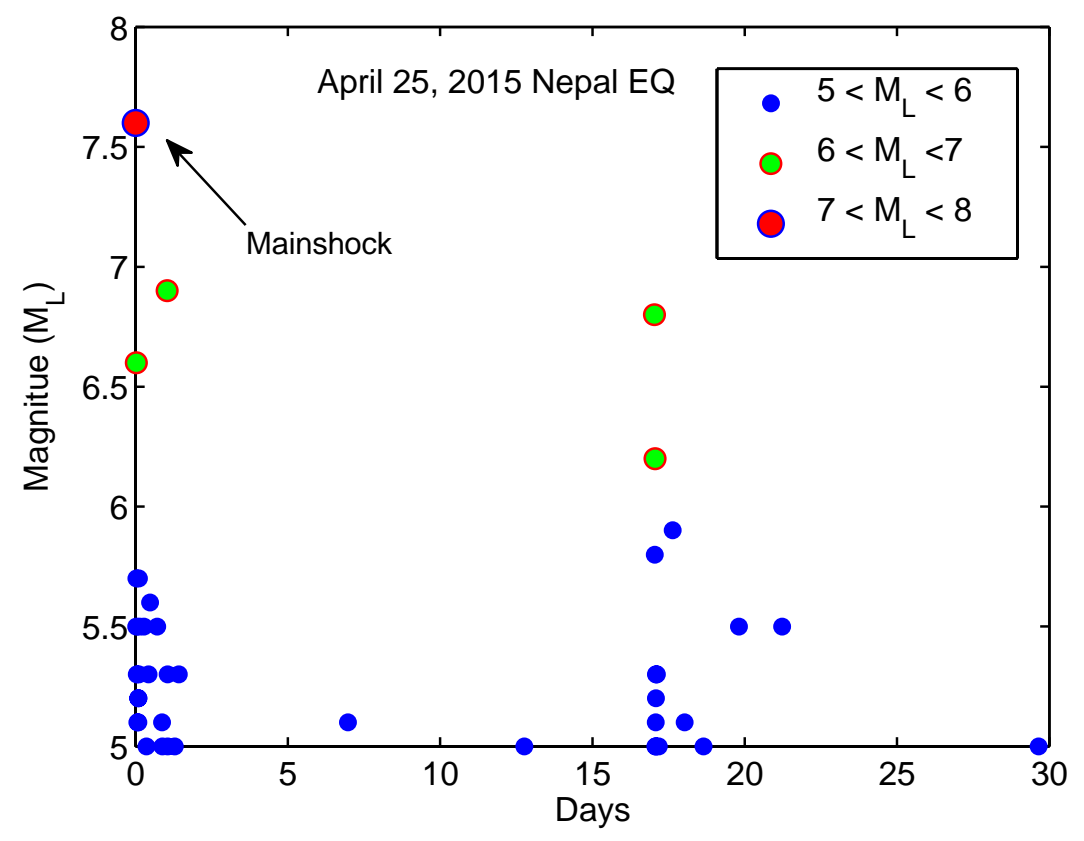

(a)

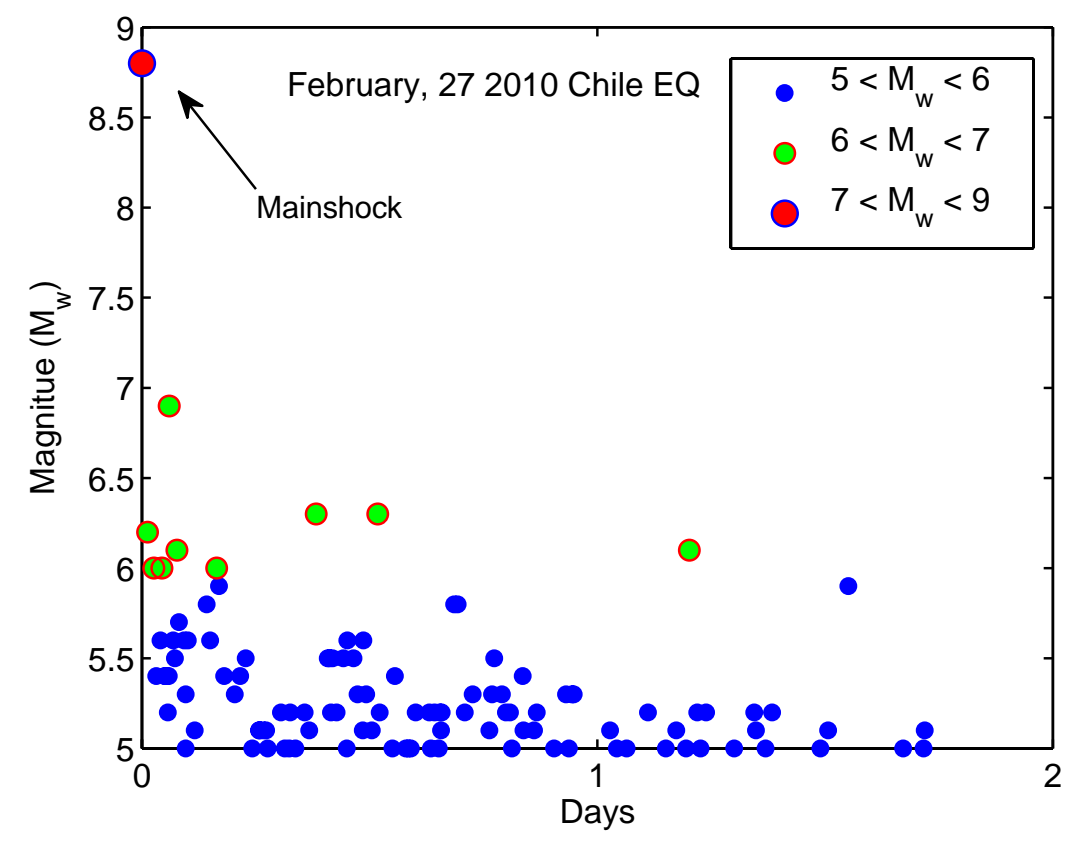

(b)

Figure 7-1 Major aftershocks (a) within 30 days of April 2015 Nepal earthquake and (b) within 2 days of February 2010 Chile earthquake 
Structures that are exposed to the combined effect of a mainshock and a series of aftershocks in a short duration of time have increased vulnerability to structural damage. A number of buildings that were slightly damaged by the mainshock had major damage or even collapsed during an aftershock event in the past earthquakes such as the 1994 Northridge earthquake, 2011 Great East Japan earthquake, 2010 Christchurch earthquake, 1999 Kocaeli earthquake; 2010 Haiti earthquake, 2012 Emili earthquake, and 2015 Gorkha earthquake (Hauksson and Jones 1994, Goda et al 2011, Shcherbakov et al 2012, USGS 2000, Decanini et al 2012, DesRoches 2011, Moss et al 2015, Kam et al 2011). Aftershocks usually have different frequency, amplitude, energy content, and duration than the mainshock, and therefore might require a different performance demand. Since they might occur immediately after the mainshock or several months later than the mainshock, assessing the integrity of a structure after a major earthquake and identifying any damage is critical for ensuring long-term safety of occupants.

Residual deformations sustained by a structural system after a seismic event can have significant role in post-earthquake structural performance assessment and in evaluation of potential damage (Ruiz-García J and Aguilar, 2015; Uma et al. 2010; Bojórquez and Ruiz-García 2013). Structural systems that exhibit excessive residual drifts under strong earthquake events may be demolished even if no severe damage or partial collapse exists. For example, a field investigation in Japan after 1995 Kobe earthquake revealed the effect of residual drifts on occupant and concluded that a residual drift ratio greater than $0.5 \%$ in buildings may require demotion of the structure from economic perspective (McCormick et al 2008). Similarly, Erochko et al. (2010) examined residual drift response of steel buildings through numerical studies and found that both steel moment resisting frames and buckling retrained frames show significant residual drifts (greater than $0.5 \%$ ) even under design basis earthquakes, while the residual drift values vary between 2.0 and $4.0 \%$ for maximum considered seismic hazard level (Erochko et al, 2010). They also reported that a steel building with $0.5 \%$ residual drift from an initial design level earthquake will not behave as designed under a subsequent design level earthquake, i.e. an aftershock. In another study for the building earthquake loss estimation, Ramirez and Miranda found that considering residual drift in loss estimation considerably increases the expected economic losses (Ramirez and Miranda, 2012). Hence, reducing residual drifts of structures subjected to seismic sequences can maximize post-event functionality, minimize repair costs, maintain overall structural integrity, and ensure the public safety. 


\subsection{Aftershock Performance Assessment Framework}

In recent years, several assessment procedures have been proposed to evaluate seismic performance and collapse capacity of structures against aftershocks (Jeon et al. 2015, Ribeiro et al. 2014, Ruiz-Garcia and Aguilar 2015). This study employs the following framework that consists of six steps for the aftershock performance assessment of steel frame buildings with selfcentering systems:

- $\quad$ Design and modeling of buildings: Reliable computational models that can capture the degradation in structural strength and stiffness of the steel frame elements associated with structural damages are first generated for steel buildings. The steel frame buildings are designed either as moment resisting frame or with SVDs to resist the lateral loads.

- $\quad$ Seismic sequence selection: As-recorded seismic sequences that consist of the combination of a mainshock and one aftershock are selected for dynamic analyses.

- $\quad$ Intensity measure selection: The spectral acceleration at the fundamental period of buildings $S_{a}\left(\mathrm{~T}_{1}\right)$ is selected as intensity measure (IM).

- $\quad$ Target post-mainshock performance levels: Three levels of mainshock damage based on the peak transient drift are considered for aftershock performance assessment. These performance levels can also be considered to represent three damage states: minor, moderate, and severe damage of structures under the effect of mainshock records. Here, the predefined levels of damage are selected to be $0.7 \%$ (DS1), $2.5 \%$ (DS2), and 3.75\% (DS3).

- $\quad$ Mainshock analysis: To identify the damage states for the aftershock analysis and to predict the collapse capacity of the frame structures under only mainshock records, an IDA using only mainshock ground motion records is conducted. In IDA, a structural model is subjected to a set of ground motions that are scaled to increasing intensity levels and a series of nonlinear time history analyses are conducted until the scaled ground motion causes the global dynamic instability, indicating the collapse of the structure (Vamvatsikos and Cornell 2002).

- $\quad$ Aftershock analysis: The aftershock IDA involves a series of nonlinear time history analyses with a constant scale factor for the mainshock event to specify particular 
damage state and incrementally increasing the intensity of aftershock records until seismic sequence indicate the collapse of the structure.

The collapse capacities obtained from the aftershock IDA analysis can be considered as the residual capacity of the building model damaged to a particular state during the mainshock event. In this study, the aftershock capacity associated with the demolition, which is the aftershock intensity that causes $2 \%$ residual drift, is also computed for both frames at different damage states.

\subsection{Numerical Modeling and Design of Steel Moment Frames}

In order to assess the performance of the SVD frame system in mitigating the seismic response of structures under seismic sequences, a nine-story steel moment resisting frame is selected for numerical investigations. The detail description of the selected steel frame, its design of as SMRF and SVD frames, and their modeling are provided in Section 5.2.

\subsection{Selection of Ground Motion Sequences}

A total of ten as-recorded seismic sequences are selected from the PEER NGA database (PEER, 2014) for response history analyses. Each selected seismic sequence consists of a mainshock and one corresponding aftershock ground motion chosen from the same station. The magnitudes of mainshock and aftershock events are selected to be equal to or greater than 5.0, and the peak ground accelerations (PGA) of horizontal components of the records are selected to be greater than $0.05 \mathrm{~g}$. To avoid the effect of soil-structure interaction, the selected acceleration-time histories recorded on the stations installed on the free field or low height buildings (Song et al., 2014). The list of seismic sequences used in this study and their important characteristics are shown in Table 7-1. In particular, the moment magnitude $\left(M_{w}\right)$, peak ground acceleration (PGA), mean period $\left(T_{m}\right)$ and significant duration $\left(D_{s}\right)$ for each earthquake are provided in the table. $T_{m}$ was

proposed by Rathje et al. (1998) as the best parameter that describes the frequency content of the ground motion and is calculated as: 


$$
T_{m}=\frac{\sum C_{i}^{2} / f_{i}}{\sum C_{i}^{2}}
$$

where $C_{i}$ is the Fourier amplitude and $f_{i}$ is the discrete Fourier transform frequencies between $0.25 \mathrm{~Hz}$ and $20 \mathrm{~Hz}$, and $f$ is frequency interval for which the Fourier transform is performed. $D_{s}$ is defined as the interval of the time over which $5 \%$ to $95 \%$ of the total Arias intensity is accumulated. Figure 7-2 shows the 5\%-damped acceleration response spectrum of the individual earthquakes. The acceleration time histories of the seismic sequences S1 and S3 are also shown in Figure 7-3. Note that a time gap of 40 seconds by adding zero acceleration values between the mainshock and aftershock records is considered to ensure the stabilized response under the free vibration of structures before the application of the aftershock.

As can be also seen from Table 7-1 and Figures 7-2 and 7-3, an aftershock might have different characteristics than the corresponding mainshock. In particular, aftershocks are usually characterized with shorter duration and higher frequency content than the mainshock (Song et al 2014). Although usually magnitude of aftershocks is smaller than that of mainshocks, they might have PGAs larger than that of mainshocks as shown in Figure 7-3(a). 
Table 7-1 Seismic sequences and their characteristics

\begin{tabular}{|c|c|c|c|c|c|c|c|}
\hline No & Earthquake & Station Name & RSN & $M_{w}$ & $\begin{array}{c}\text { PGA } \\
(\mathrm{g})\end{array}$ & $\begin{array}{l}T_{m} \\
(\mathbf{s})\end{array}$ & $\begin{array}{l}D_{s} \\
(\mathbf{s})\end{array}$ \\
\hline \multirow[t]{2}{*}{ S1 } & \multirow[t]{2}{*}{1987 Whittier } & Mt Wilson - CIT Seis Sta & 663 & 6.0 & 0.122 & 0.19 & 9.8 \\
\hline & & Mt Wilson - CIT Seis Sta & 715 & 5.3 & 0.145 & 0.21 & 3.9 \\
\hline \multirow[t]{2}{*}{$\mathrm{S} 2$} & \multirow{2}{*}{$\begin{array}{l}1980 \text { Irpinia, } \\
\text { Italy }\end{array}$} & Calitri & 289 & 6.9 & 0.126 & 2.62 & 24.2 \\
\hline & & Calitri & 300 & 6.2 & 0.154 & 2.48 & 20.0 \\
\hline \multirow[t]{2}{*}{ S3 } & \multirow{2}{*}{$\begin{array}{l}1999 \text { Chi-Chi, } \\
\text { Taiwan }\end{array}$} & CHY035 & 1202 & 7.6 & 0.251 & 0.85 & 27.9 \\
\hline & & CHY035 & 2709 & 6.2 & 0.136 & 0.64 & 12.1 \\
\hline \multirow[t]{2}{*}{ S4 } & 1994 & Castaic-Old Ridge Route & 963 & 6.7 & 0.568 & 0.54 & 9.1 \\
\hline & Northridge & Castaic-Old Ridge Route & 1676 & 5.9 & 0.138 & 0.47 & 9.7 \\
\hline \multirow[t]{2}{*}{ S5 } & \multirow{2}{*}{$\begin{array}{l}1986 \text { Chalfant } \\
\text { Valley }\end{array}$} & Zack Brothers Ranch & 547 & 5.8 & 0.272 & 0.42 & 11.5 \\
\hline & & Zack Brothers Ranch & 558 & 6.2 & 0.447 & 0.48 & 8.1 \\
\hline \multirow[t]{2}{*}{ S6 } & \multirow[t]{2}{*}{1983 Coalinga } & Pleasant Valley P.P. & 367 & 6.4 & 0.300 & 0.61 & 11.6 \\
\hline & & Pleasant Valley P.P. & 412 & 5.8 & 0.575 & 0.41 & 7.3 \\
\hline \multirow[t]{2}{*}{ S7 } & 1980 & Convict Creek & 230 & 6.1 & 0.419 & 0.33 & 7.1 \\
\hline & $\begin{array}{l}\text { Mammoth } \\
\text { Lake }\end{array}$ & Convict Creek & 248 & 5.9 & 0.266 & 0.39 & 2.8 \\
\hline \multirow[t]{2}{*}{ S8 } & 1979 Imperial & El Centro Array \#3 & 178 & 6.5 & 0.223 & 0.47 & 14.1 \\
\hline & Valley & El Centro Array \#3 & 201 & 5.0 & 0.097 & 0.33 & 5.3 \\
\hline \multirow[t]{2}{*}{ S9 } & 1980 & San Ramon-Eastman Kodak & 214 & 5.8 & 0.150 & 1.00 & 14.2 \\
\hline & Livermore & San Ramon-Eastman Kodak & 223 & 5.4 & 0.280 & 0.59 & 12.4 \\
\hline \multirow[t]{2}{*}{ S10 } & 1994 & Moorpark - Fire Sta & 1039 & 6.7 & 0.193 & 0.58 & 16.1 \\
\hline & Northridge & Moorpark - Fire Sta & 1681 & 5.9 & 0.140 & 0.55 & 10.0 \\
\hline
\end{tabular}



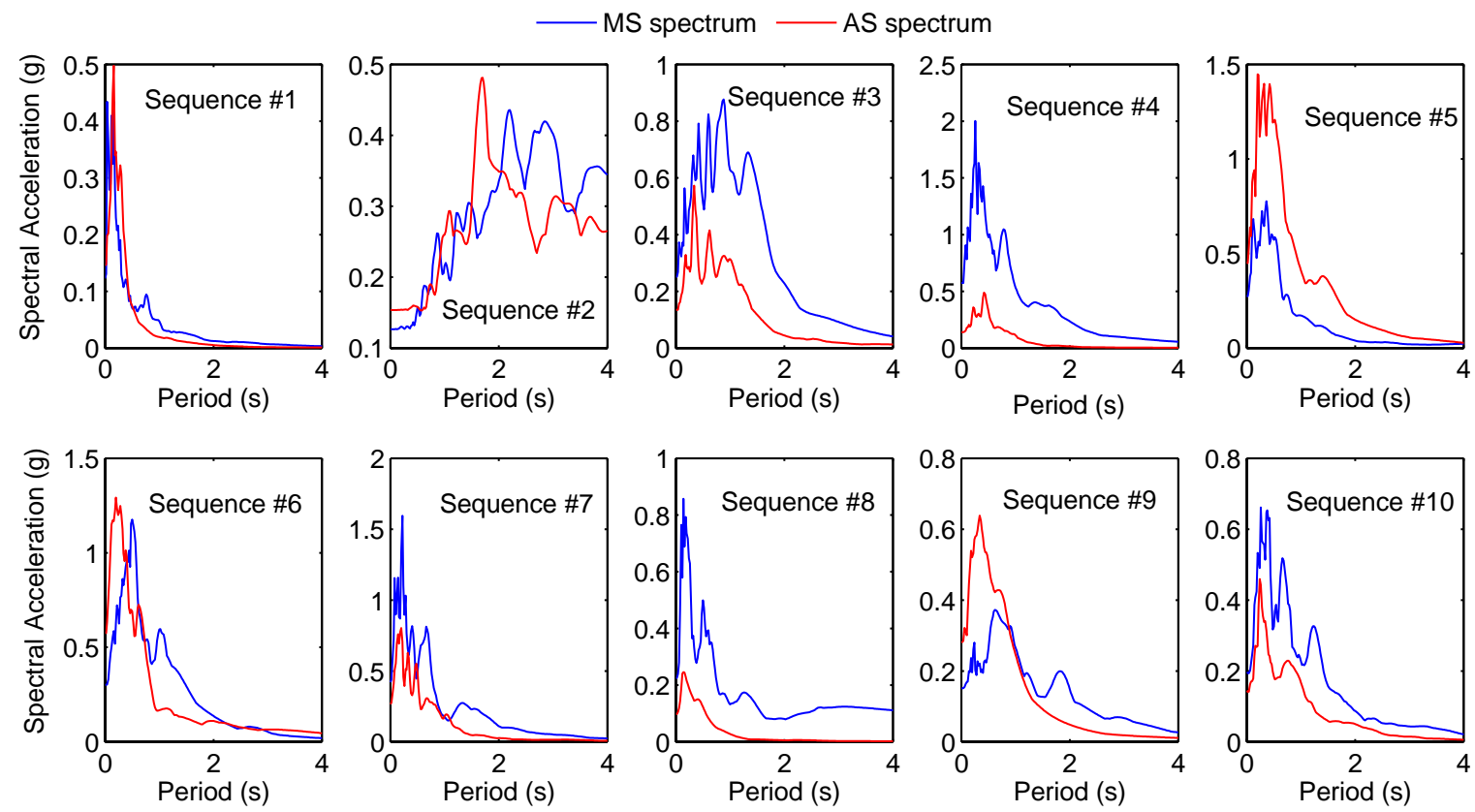

Figure 7-2 Response spectrum of selected seismic sequences
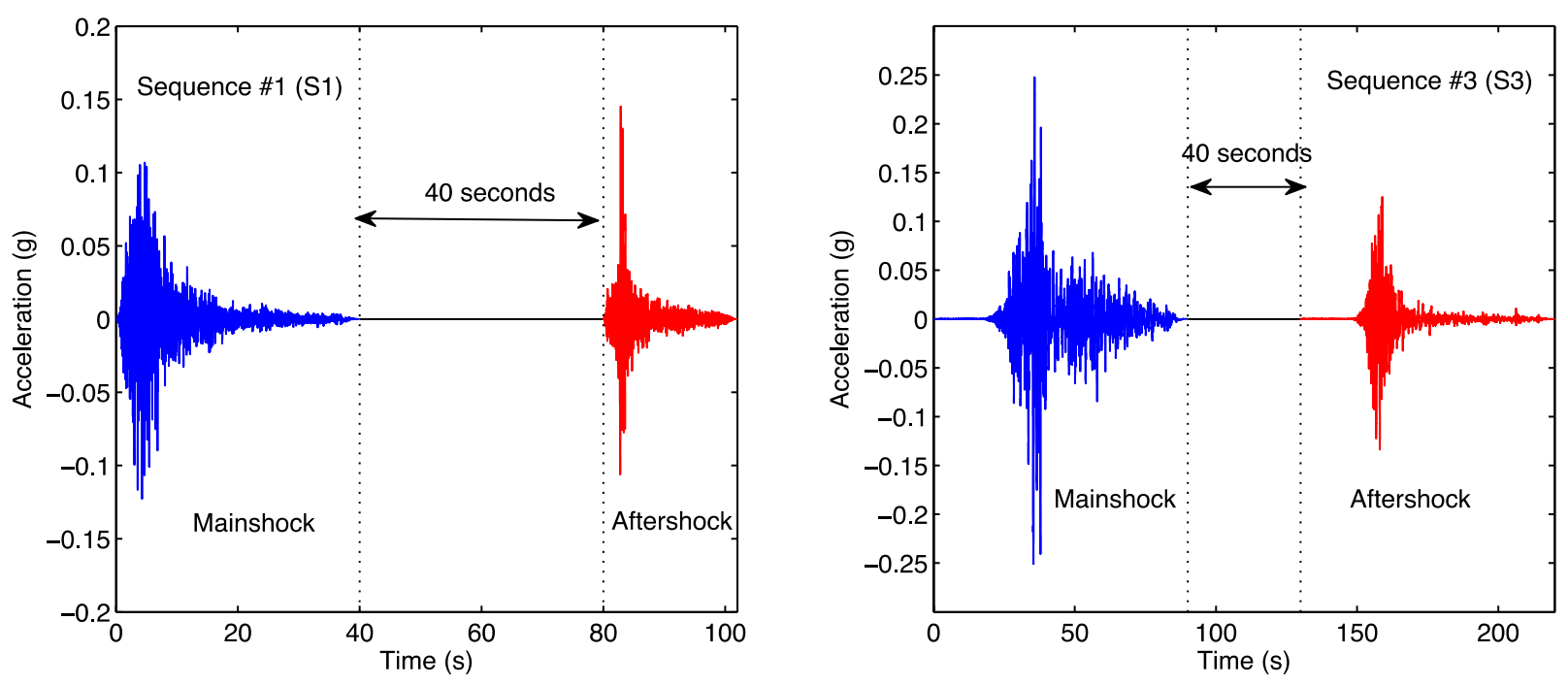

Figure 7-3 Acceleration time history for a mainshock-aftershock sequence recorded at Convict Creek Station from 1980 Mammoth Lake Earthquake 


\subsection{Performance Assessment for Mainshock-Aftershock Analysis}

\subsubsection{Mainshock Seismic Assessment}

In order to determine the collapse capacity of the frames under only mainshocks, the mainshock IDA analyses are conducted on the structural models. Considering the limitations in computational modeling and simulations as well as inadequate resistance of the columns beyond $5 \%$ drift limit, a potential collapse limit state corresponding to $5 \%$ inter-story drift is selected as suggested by previous studies (Hsiao et al., 2013). Figure 7-4 illustrates the IDA curves that show the relationship between spectral acceleration and the maximum inter-story drift ratio (MIDR) for individual mainshock records as well as the median response for the SMRF and SVD frames. Each dot on an IDA curve indicates the response for an individual earthquake scaled to a specific ground motion intensity level. Median collapse capacity of the frames can be calculated as the ground motion intensity where half of the selected mainshock records cause collapse of a structural model (Song et al 2014). The collapse capacities from the mainshock IDA are $0.50 \mathrm{~g}$ and $0.75 \mathrm{~g}$ for the SMRF and SVD frames, respectively. The result indicates the superior collapse capacity of the frame with the installed SVDs under mainshock records.

The damage sustained during the mainshock can significantly affect the performance of the structure at subsequent seismic events (Ruiz-García J and Aguilar, 2015; Uma et al. 2010; Bojórquez and Ruiz-García 2013). Before conducting the aftershock IDA analysis, building models are subjected the mainshock records that are scaled to achieve a damage state corresponding to $0.7 \%, 2.5 \%$, or $3.75 \%$ drift. The results of the mainshock IDA analysis are used to determine the required scale factors to achieve corresponding damage state during the aftershock analysis that is discussed next. 

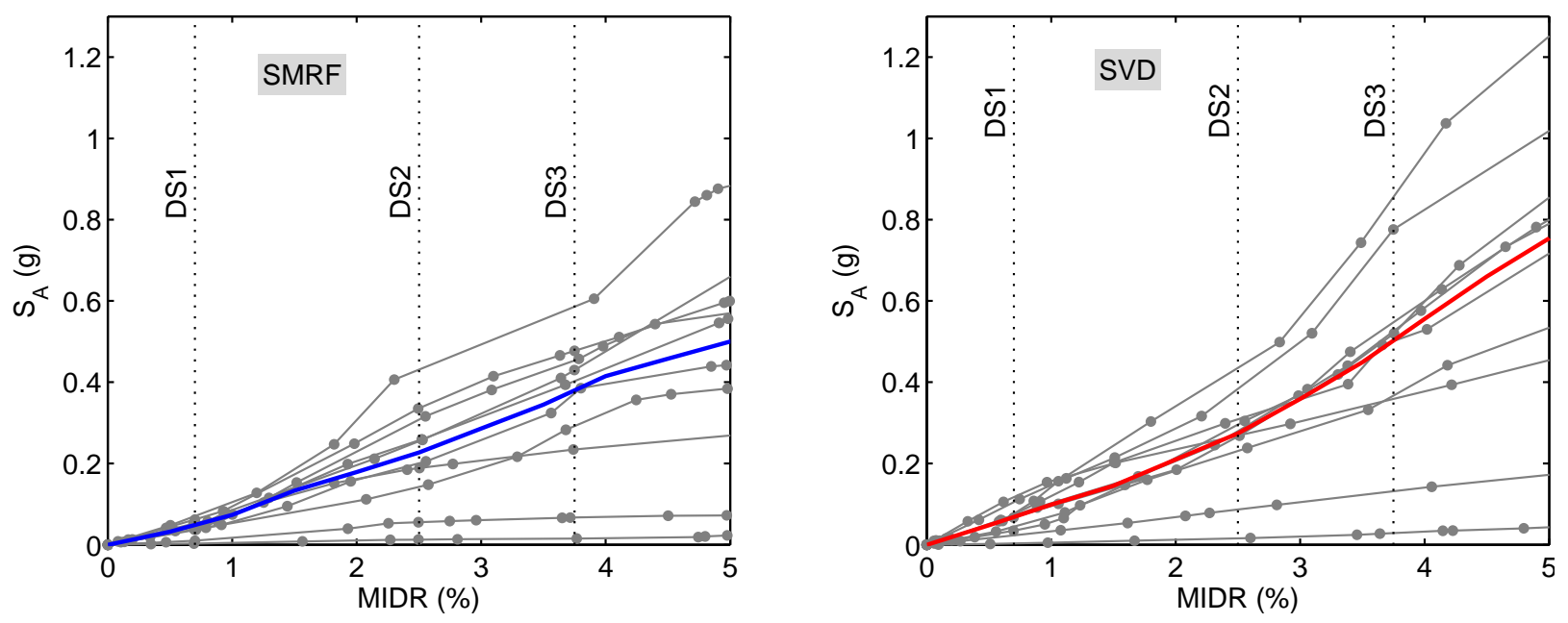

Figure 7-4 Incremental dynamic analysis curves of frames subjected to mainshocks: (a) SMRF, and (b) SVD frames

\subsubsection{Aftershock Seismic Assessment}

Nonlinear response history analyses of the undamaged SMRF and SVD buildings are conducted first under a given mainshock record scaled to a damage state of interest. Note that to reach a particular damage state in the SMRF and SVD frames under a mainshock event, different scale factors are used during the simulation of SMRF and SVD frames. In particular, a larger scaling factor, especially for DS3 simulations, is usually applied to a given mainshock for the SVD frame analysis. Then, the corresponding aftershock event is applied to the damaged structural model with the elapsed time of 40 seconds at the end of mainshock event to ensure the building to come to rest. To develop the aftershock IDA curve, the nonlinear time history analyses are repeated with increasing intensity measure of aftershock to represent structural behavior until global dynamic instability.

Aftershock IDA curves for the SMRF and SVD frames subjected to different damage states during mainshock are shown in Figure 7-5. The figure illustrates the relationship between the spectral acceleration of the aftershock and the maximum inter-story drift ratio experienced by the structures during the mainshock-aftershock sequence. The results for the individual earthquakes 
as well as the median response are shown in each plot. It can be seen that the median aftershock IDA curves for the SMRF and SVD frames feature a steep line at low intensity levels of aftershock events, indicating the maximum drift of the frames is controlled by the mainshock event for low intensity aftershocks. This initial steep increase in the spectral acceleration values in the aftershock IDA curves becomes more pronounced with the increasing damage sustained during the mainshock.
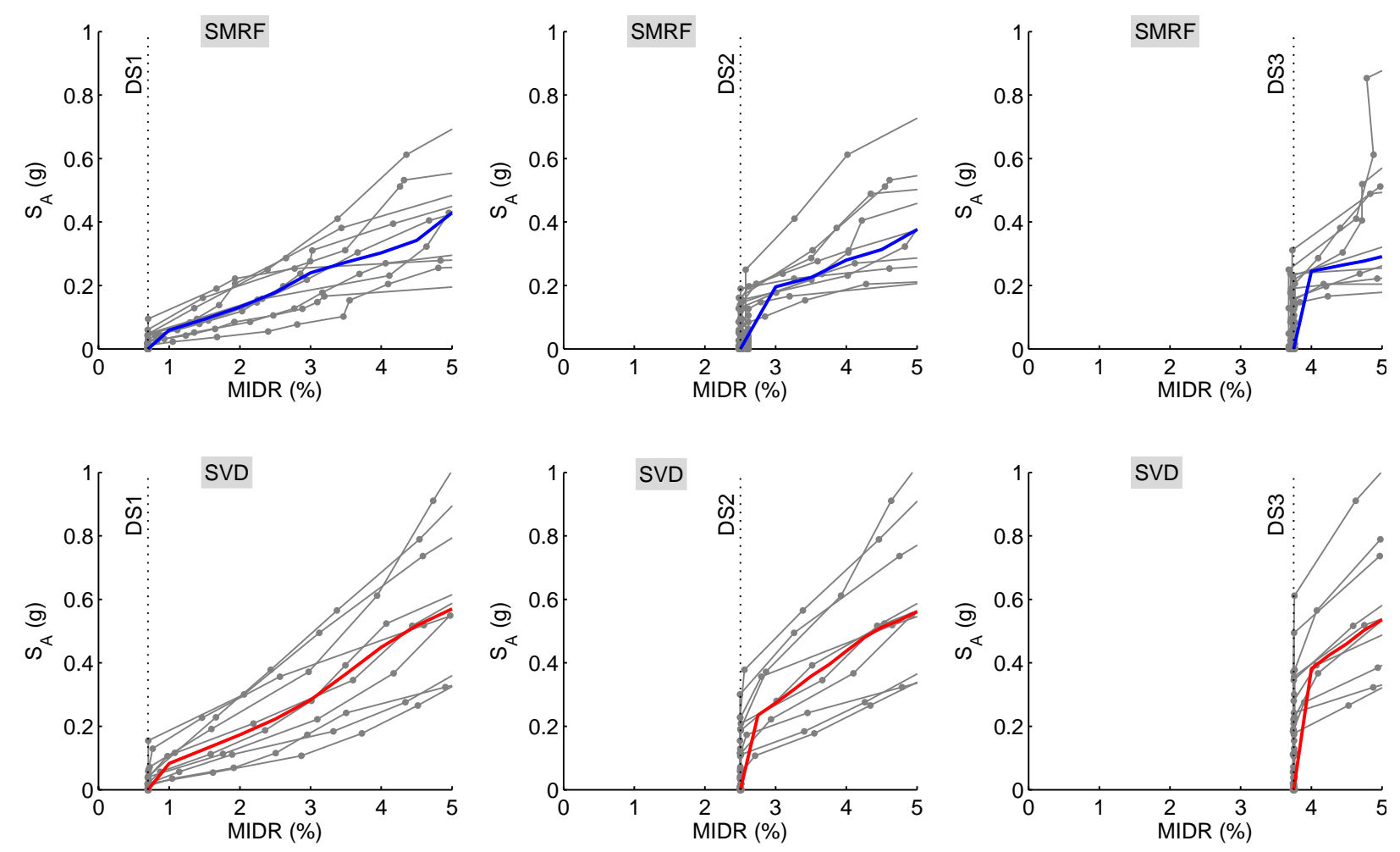

Figure 7-5 Maximum inter-story drift versus spectral acceleration IDA curve under mainshock-aftershock sequence indicating three damage states for the SMRF and SVD frames

Figure 7-6 shows the median aftershock IDA curves at three damage states for both SMRF and SVD frames. Figure 7-6 provides the median collapse capacities of SMRF and SVD frames at each mainshock damage state. The corresponding IDA curve and collapse capacities for the 
mainshock only are also provided in Figures 7-4 and 7-6, respectively for comparison purposes, which also presents the change in the median collapse capacity for the building models subjected to mainshock-aftershock sequences compared to these subjected to mainshock only. For the SMRF, the median collapse capacity of the building with sustained mainshock damages of DS1, DS2, and DS3 decreases 14\%, 25\%, and 42\%, respectively. This indicates the SMRF with low damages from the mainshock experiences somewhat small reductions in its collapse capacity during future earthquakes, while the effect of aftershocks on the collapse capacity becomes more pronounced when the structural damage during the mainshock increases. Similar observations for the steel frame buildings were reported in previous studies (Li et al., 2014; Ribeiro et al. 2014).

Table 7-2 Median collapse capacities for SMRF and SVD under seismic sequences

\begin{tabular}{cccccccccc}
\hline & \multicolumn{3}{c}{ SMRF } & \multicolumn{5}{c}{ SVD } \\
\cline { 2 - 8 } & $\begin{array}{c}\text { Mainshock } \\
\text { Only }\end{array}$ & DS1 & DS2 & DS3 & $\begin{array}{c}\text { Mainshock } \\
\text { Only }\end{array}$ & DS1 & DS2 & DS3 \\
\cline { 2 - 9 } & 0.50 & 0.43 & 0.38 & 0.29 & 0.75 & 0.57 & 0.56 & 0.54 \\
$\begin{array}{c}\text { Median collapse } \\
\text { capacity (g) } \\
\begin{array}{c}\text { Decrease in } \\
\text { collapse capacity }\end{array}\end{array}$ & - & $14 \%$ & $25 \%$ & $42 \%$ & - & $24 \%$ & $26 \%$ & $29 \%$ \\
\hline
\end{tabular}




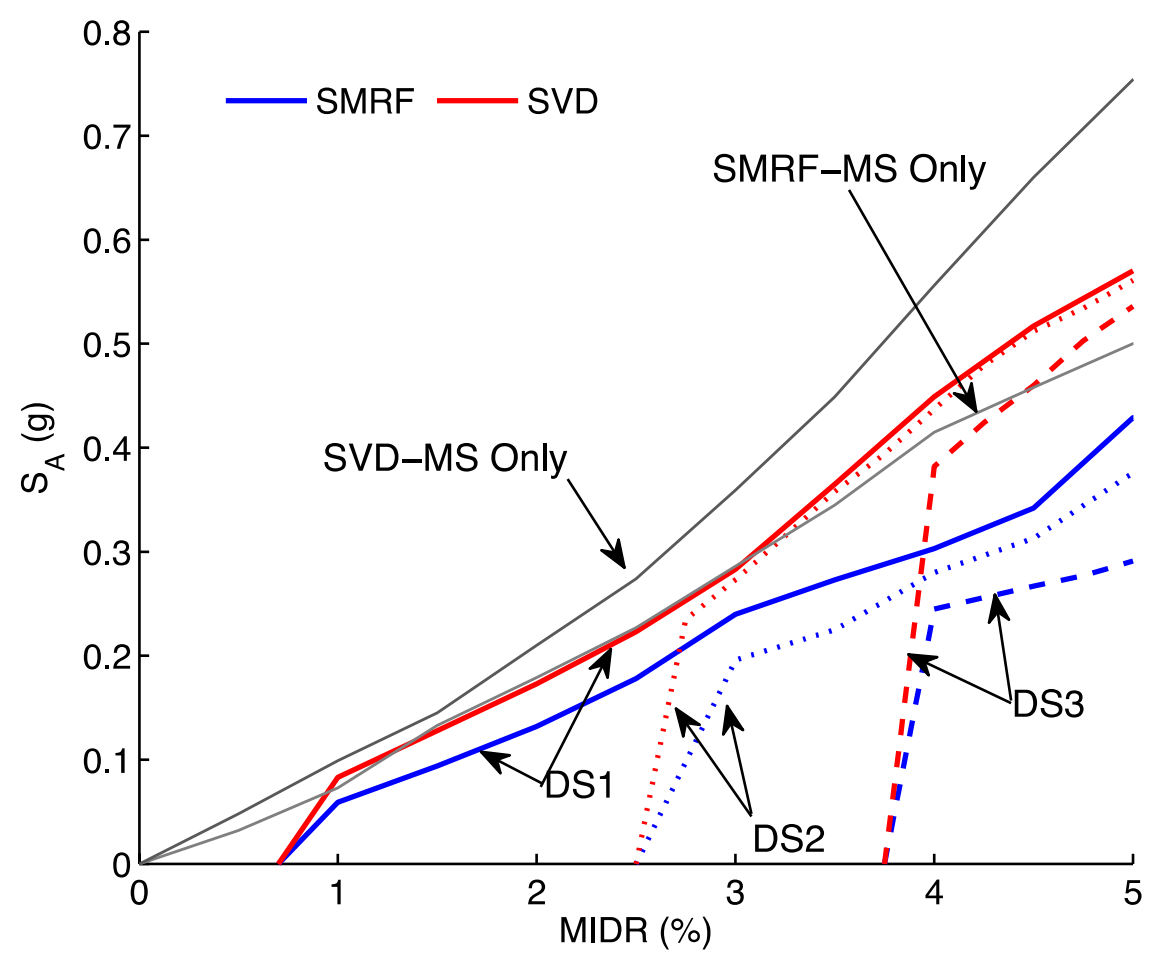

Figure 7-6 Median IDA curves at three damage states for SMRF and SVD frame under seismic sequences

The steel frame with the installed SVDs reaches higher median collapse capacities at all three damage states compared to the SMRF. Similar to the SMRF, the median collapse capacity of the mainshock-damaged SVD frame decreases compared to that of the SMRF frame subjected to only mainshock events. However, the reduction in the collapse capacity does not increase significantly with the increasing mainshock damage level. Compared to the SMRF, the SVD frame provides $33 \%, 49 \%$, and $84 \%$ higher collapse capacity than the SMRF for the DS1, DS2, and DS3 mainshock-damaged buildings. The superior collapse performance of the SVD frame can also be seen from Figure 7-7, which compares the aftershock fragility curves of the SMRF and SVD frames at DS1, DS2, and DS3 levels. The aftershock collapse fragility curves are modeled using a lognormal distribution and are quantified by the median collapse capacity and lognormal standard deviation of the intensity measure of the ground motion records. 

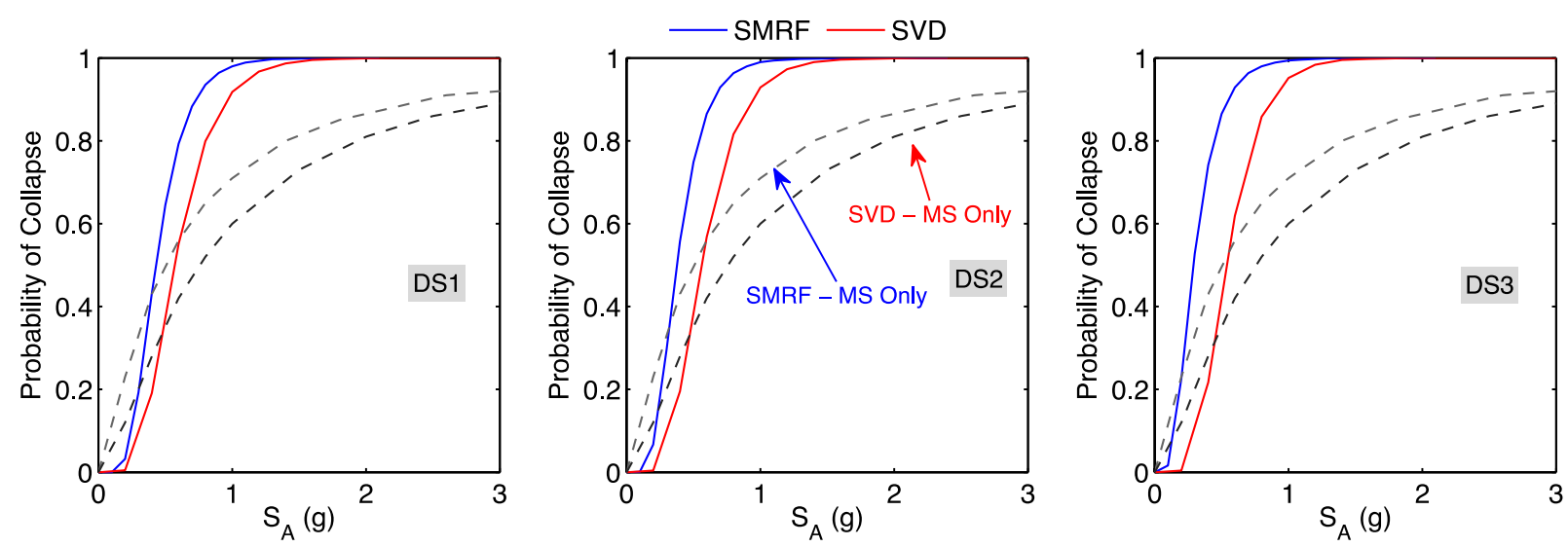

Figure 7-7 Comparative fragility curves for SMRF and SVD frames at three damage states: (a) DS1, (b) DS2, and (c) DS3

\subsubsection{Demolition Capacity Assessment}

Controlling residual drift of steel structures is another important performance objective. According to FEMA P-58 (FEMA, 2012), steel structures that experience peak residual drifts greater than $1 \%$ require major realignment and those with permanent drifts over $2 \%$ cannot be repaired. One recent study reported that the aftershock capacity associated with the demolition (i.e. associated to a $2 \%$ residual drift) provides better parameter to measure the seismic performance of structures subjected to aftershock sequences (Ruiz-García and Aguilar 2015). Here, the aftershock capacity associated with the demolition is also evaluated for the steel buildings designed with and without SVDs and sustained different damage levels from the mainshock. Figures Figure 7-8(a) and (b) illustrate the relationship between the spectral acceleration and the median residual drift ratio for the SMRF and SVD frames subjected to different mainshock damages. It can be seen that the aftershock capacity associated with the demolition for the SMRF are $0.30 \mathrm{~g}$ for DS1 and DS2 levels, and $0.18 \mathrm{~g}$ for DS3 level. Note that these values are considerably lower than the collapse capacity of the SMRF at each of these damage states. However, for the SVD frame, the residual drifts barely exceed $1 \%$ at the collapse capacity of the structure. This indicates the SVD frame has 
the potential to lower the post-earthquake losses by minimizing the residual drifts and thereby satisfying a reparability state.

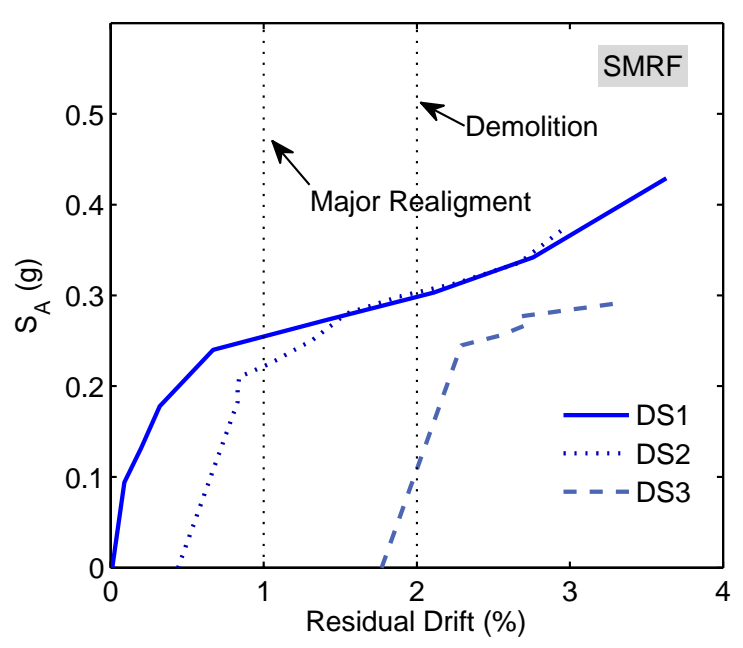

(a)

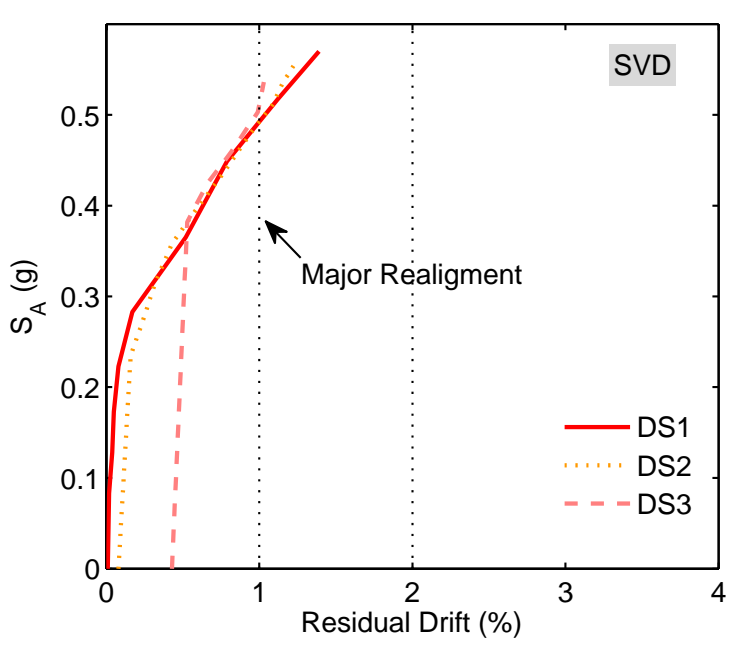

(b)

Figure 7-8 Median aftershock IDA residual drift curves for SMRF and SVD frames at different mainshock damage levels

\subsubsection{Effect of Aftershock Polarity}

To assess the effect of ground motion polarity on the damage potential of seismic sequences for the SMRF and SVD frames, the aftershock motions are also applied in the negative direction, and aftershock IDA analysis are repeated. The results are presented for three cases: (a) positive polarity (PP); (b) negative polarity (NP); and (c) maximum response (MAX). In all cases the original direction of the mainshock is preserved, while the aftershock is applied in the same and the opposite directions of the as-recorded acceleration time history to get the response for the cases PP and NP, respectively. The higher response obtained from the cases PP and NP is selected for each individual ground motion to get the MAX.

The first story drift histories of the SMRF and SVD frames subjected to mainshockaftershock sequence 2 are illustrated in Figure 7-9. Here, the mainshock record is scaled such that the SMRF or SVD frame reaches to DS2 or DS3, and then the aftershock record is applied to the 
SMRF frame with a scaling factor of 0.70 and to the SVD frame with a scaling factor of 1.60. For the SMRF frame, when the mainshock is scaled to DS2, there is no significant residual drift and a strong aftershock with PP or NP produces similar residual drifts. However, the mainshock scaled to DS3 level causes a residual drift of $1.3 \%$ at the end of the mainshock and the polarity of the aftershock significantly affect the aftershock response. The aftershock with PP produces large story drifts and induces further permanent drifts up to $3.7 \%$, while the aftershock with NP lead to a re-centering behavior and reduce the residual drift to $0.3 \%$. On the other hand, the SVD frame does not have significant residual drift upon aftershock at both DS2 and DS3 levels and therefore the aftershocks with different polarities produce similar peak story drift and residual drifts.
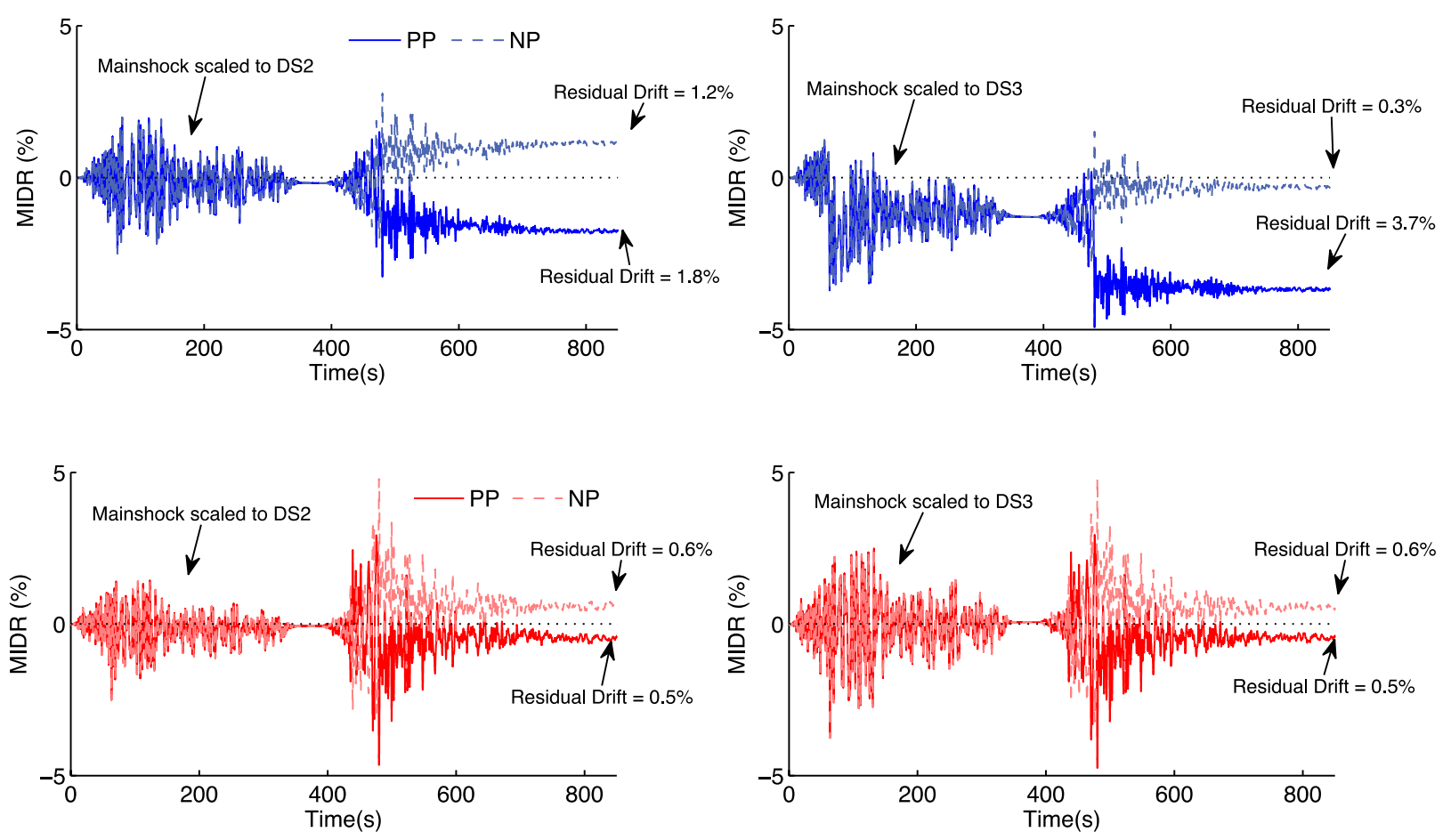

Figure 7-9 Time histories of fist floor drift for (a) SMRF and (b) SVD frames subjected to sequence S2

To further explore the aftershock ground motion polarity on the aftershock response, the median IDA curves for the SMRF and SVD frames at three damage states are shown in Figures 7- 
10 and 7-11 for the MIDR and peak residual drift, respectively. It can be seen that the polarity of aftershock records does not affect both median MIDR and residual drift response at low mainshock damage level (DS1) for the SMRF. However, with the increasing mainshock damage level, the IDA curves are considerably influenced with the aftershock polarity. On the other hand, the effect of polarity on the aftershock MIDR is minimal for the SVD frame even at high mainshock damage levels. Considering the effect of polarity amplifies the residual drift response for the SVD at DS3 while it does not cause a significant change at other damage levels.
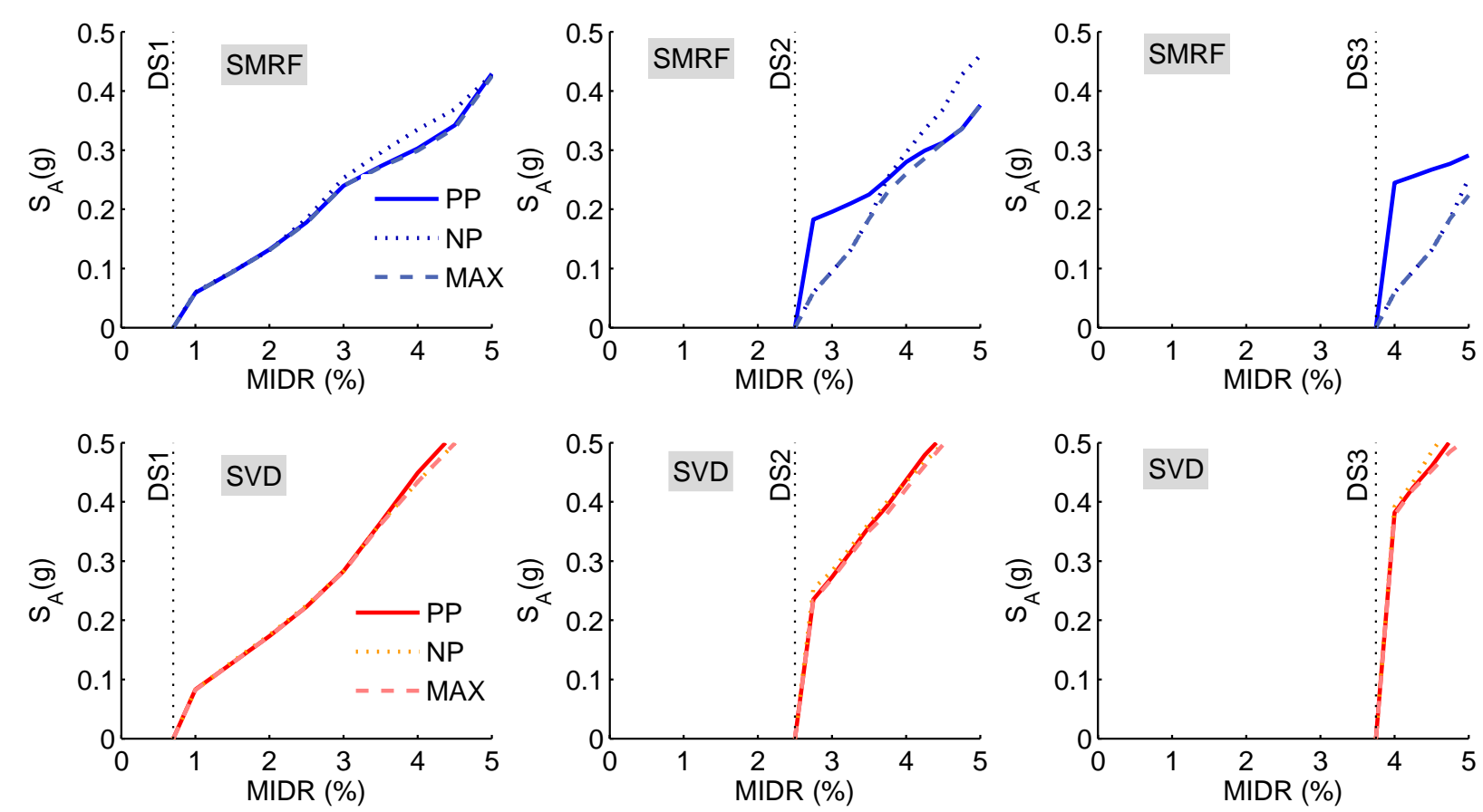

Figure 7-10 Median aftershock IDA curves for maximum inter-story drift for SMRF and SVD frames at different mainshock damage levels considering aftershock polarity 

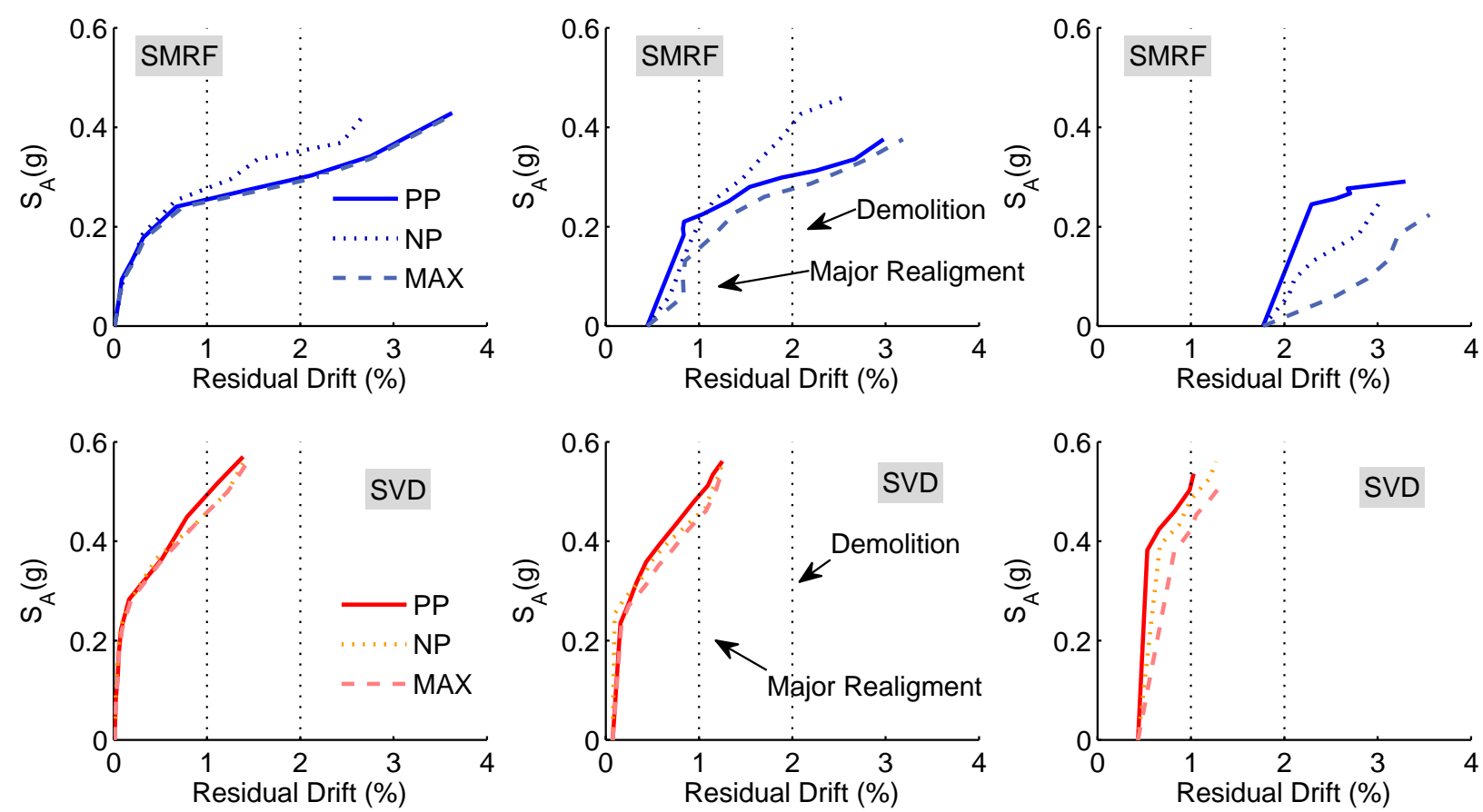

Figure 7-11 Median aftershock IDA curves for maximum residual drift for SMRF and SVD frames at different mainshock damage levels considering aftershock polarity

\subsection{Closure}

In this section, the aftershock performance of steel frame buildings with and without installed superelastic viscous dampers is investigated. A case study building is designed and modeled with and without dampers. The models capture the degradation in the structural strength and stiffness of the steel frame elements associated with structural damages. First, incremental dynamic analysis is conducted for both frames to identify the structural response under mainshock events scaled to increasing intensities. A suite of historical mainshock and aftershock ground motion records are employed in the analysis. Three post-mainshock damage states are defined based on maximum inter-story drift thresholds and the corresponding aftershock collapse fragility analyses are performed at each damage state. 
Results shows that the SVD frame has higher median collapse capacities than the SMRF for undamaged (considering only mainshock) and damaged conditions. When the aftershocks are considered, the collapse capacities of both SMRF and SVD frames decrease. For the SMRF, the reduction in the collapse capacity is more pronounced when the building experiences a higher mainshock damage. However, the intensity of mainshock does not affect the aftershock collapse performance of the SVD frame. At the largest post-mainshock damage state, the SVD frame provides $86 \%$ higher collapse capacity compared to the SMRF. The aftershock capacities associated with the demolition is considerably lower than the collapse capacity for the SMRF frame at all damage states, while the SVD frame successfully control the residual drifts and mostly limits the residual drifts below $1 \%$. It is also found that the polarity of the aftershock records does not influence the performance of the SVD frame due to its ability to limit the residual drifts at the end of a mainshock event. These results indicate the advantages of self-centering systems in reducing the seismic losses and risks in steel structures when they are subjected to seismic sequences. 



\section{EFFECT OF TEMPERATURE ON SEISMIC PERFORMANCE OF SVDS}

\subsection{Introduction}

This section explores the effects of temperature on the seismic performance of steel frame buildings designed with SVDs. Since the mechanical behavior of SMAs and high damped butyl rubber is highly dependent on temperature, it is important to study the effect of temperature on the performance of SVDs. First, the influence of temperature on the hysteretic response of butyl rubber and SMAs are discussed through experimental test results. Next, nonlinear time history analyses of a nine-story steel moment resisting frame building with installed SVDs are conducted for environmental temperature variations of $0^{\circ} \mathrm{C}, 23^{\circ} \mathrm{C}$, and $40^{\circ} \mathrm{C}$. A total of 10 far-field ground motion records are employed in the simulations. Results are analyzed in terms of peak story drift and peak absolute acceleration. The variations of peak inter-story drift ratio, which is correlated with damage in structural elements, and peak absolute floor acceleration, which is correlated with damage in non-structural components, with temperature are studied.

\subsection{Temperature Effects on Butyl Rubber}

In order to assess the effect of temperature on the behavior of HD butyl rubber, static and dynamic shear tests are conducted at Gannon University. The HD butyl is 50 durometer Shore A. All tests are performed according to ASTM D5992. The specimen geometry is a double shear with two identical rubber elements symmetrically disposed on opposite sides of a central rigid member. This specimen geometry is specified in ASTM D5992 and carefully designed to yield a length/wall thickness ratio of 8 (=1.6"/.2") to achieve a state of pure shear. All testing is performed on an MTS 810 servo-hydraulic test system. An environmental chamber is used to create desired test temperatures.

The tests are conducted at $100 \%$ shear strain at $1 \mathrm{~Hz}$ loading frequency. This strain level is selected to yield useful information about temperature impact as higher strain levels tend to be dominated by nonlinear effects. The tests are performed at $0^{\circ} \mathrm{C}, 23^{\circ} \mathrm{C}$ and $40^{\circ} \mathrm{C}$. Figure $8-1$ shows

the hysteresis loops at various temperatures for the HD butyl rubber. In addition, to enable the 
evaluation of the test results in a quantitative way, the equivalent stiffness and loss factor are calculated. They are defined as:

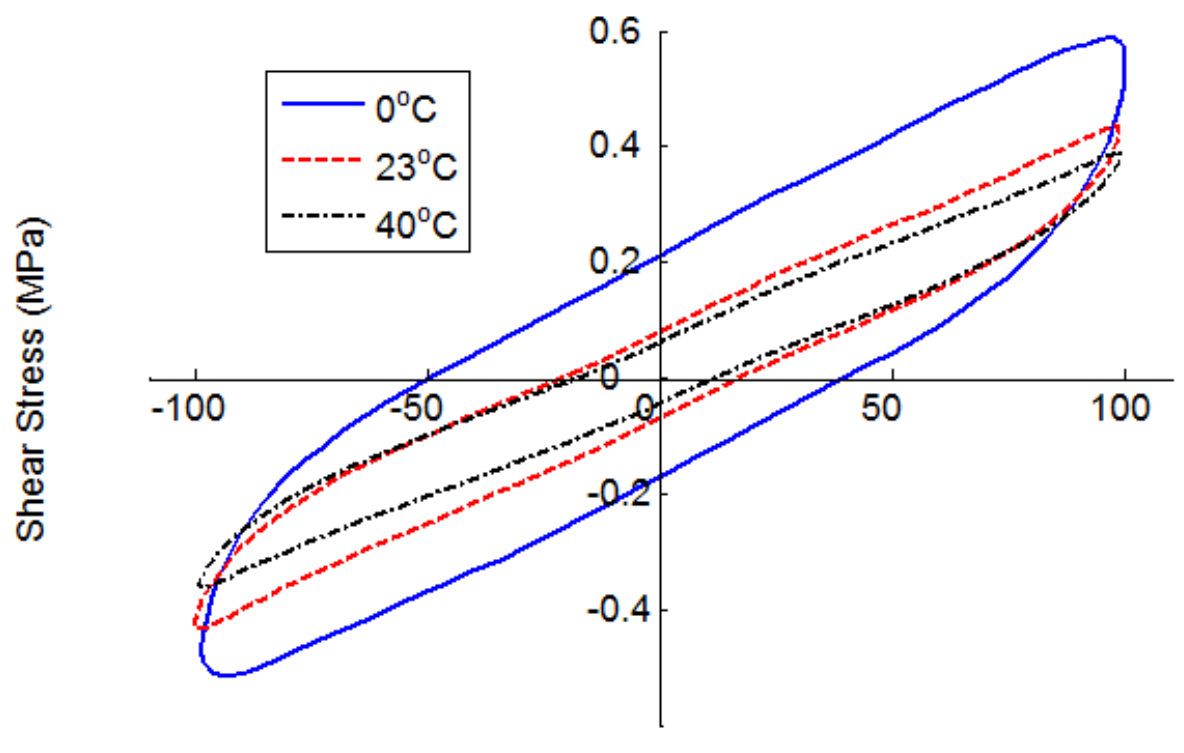

Shear Strain (\%)

Figure 8-1 Shear stress - shear strain curves of HD butyl rubber at different temperatures

$$
\begin{gathered}
K_{s}=\frac{F_{\text {max }}-F_{\text {min }}}{d_{\text {max }}-d_{\text {min }}} \\
\eta=\frac{1}{2 \pi} \frac{E_{D}}{E_{S}}
\end{gathered}
$$

where $E_{D}$ is the energy dissipated per cycle (hysteresis area), $E_{S}$ is the maximum strain energy for the same cycle calculated as the energy absorbed in a linear system that has the same maximum displacement and force, $F_{\max }$ and $F_{\min }$ are the maximum and minimum forces attained for the maximum and minimum cyclic displacements $d_{\max }$ and $d_{\min }$. Figure 8-2 illustrates the variation of equivalent stiffness and loss factor with temperature. The results reveal that both the equivalent stiffness and loss factor decreases with increasing temperature. The properties seem to be more 
temperature sensitive when the temperature decreases below room temperature compared to an increase in temperature above room temperature. In particular, the equivalent stiffness and loss factor vary $+21 \%$ and $+125 \%$, respectively when the temperature changes from room temperature to $0^{\circ} \mathrm{C}$. On the other hand, the change in the equivalent stiffness and loss factor are only $-13 \%$ and $-23 \%$ when the temperature is increased from $23^{\circ} \mathrm{C}$ to $40^{\circ} \mathrm{C}$.
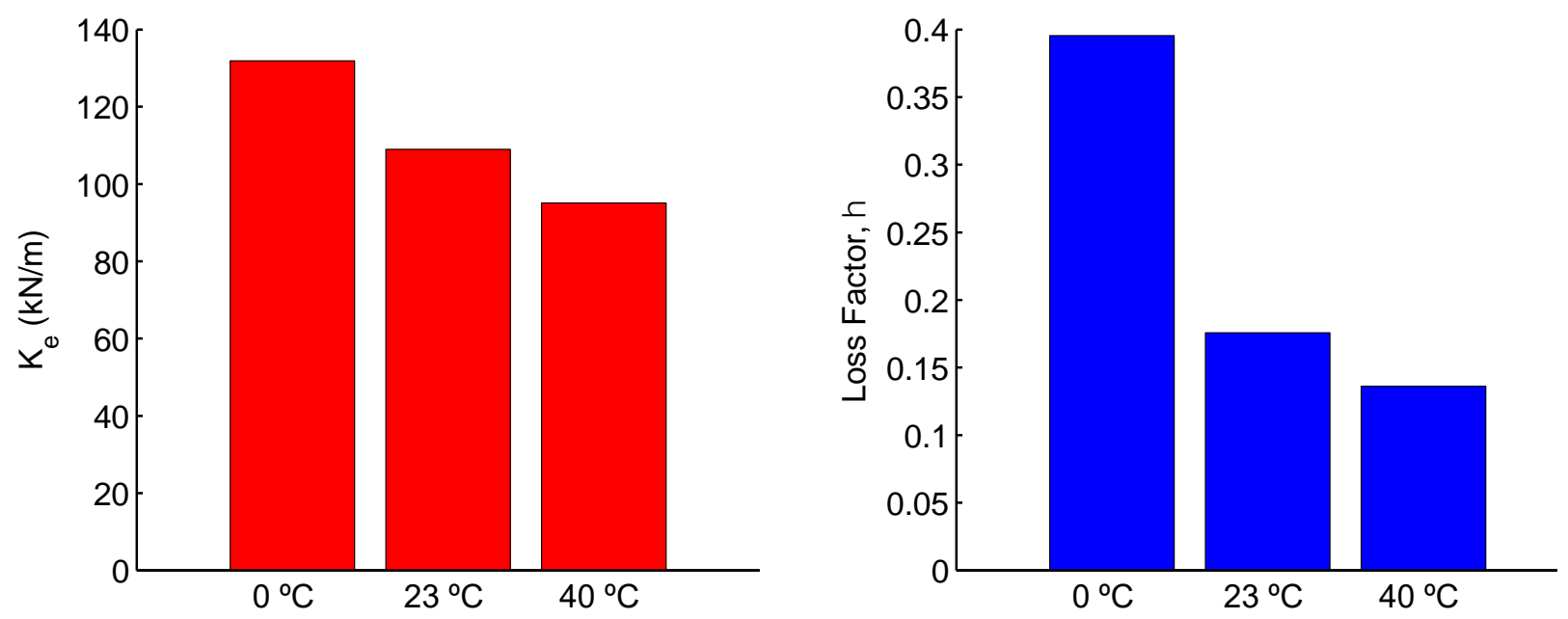

Figure 8-2 Variation of equivalent stiffness and loss factor for HD butyl with temperature

\subsection{Temperature Effects on Shape Memory Alloys}

In order to investigate the temperature dependence of superelastic behavior of NiTi SMAs, the experimental tests are conducted at different temperatures. The material used is NiTi wires with a diameter of $1.5 \mathrm{~mm}$ and obtained from SAES Smart Materials. The alloy chemical composition has $55.8 \%$ nickel by weight and the balance titanium. The austenite start and finish temperatures are specified by manufacturer as $A_{s}=-10^{\circ} \mathrm{C}$ and $A_{f}=5^{\circ} \mathrm{C}$, respectively. An MTS servo-hydraulic load frame are used to conduct tests at various frequencies and temperatures. The sinusoidal tensile tests are performed under displacement control at $1 \mathrm{~Hz}$ at $0^{\circ} \mathrm{C}, 23^{\circ} \mathrm{C}$ and $40^{\circ} \mathrm{C}$. Figure 8-3 shows strain-stress curves of NiTi wires at different temperatures. It can be seen that the hysteresis loops of SMA shifts upward with increasing temperature. Figure 8-4 illustrates the variation of modulus of elasticity and loss factor with temperature. It can be seen that the modulus 
of elasticity decreases from $42.6 \mathrm{GPa}$ at $23^{\circ} \mathrm{C}$ to $27.6 \mathrm{GPa}$ at $0^{\circ} \mathrm{C}$, recording a large decrease ($35 \%$ ) in modulus of elasticity at low temperatures. However, the modulus varies only $+10 \%$ when the temperature changes from $23^{\circ} \mathrm{C}$ to $40^{\circ} \mathrm{C}$. On the other hand, the loss factor attains higher values at lower temperatures. As temperature increases from $0{ }^{\circ} \mathrm{C}$ to $40{ }^{\circ} \mathrm{C}$, the loss factor reduces $38 \%$. If the room temperature is considered as reference, the loss factor varies $-24 \%$ at $0{ }^{\circ} \mathrm{C}$ and $+21 \%$ at $40^{\circ} \mathrm{C}$.

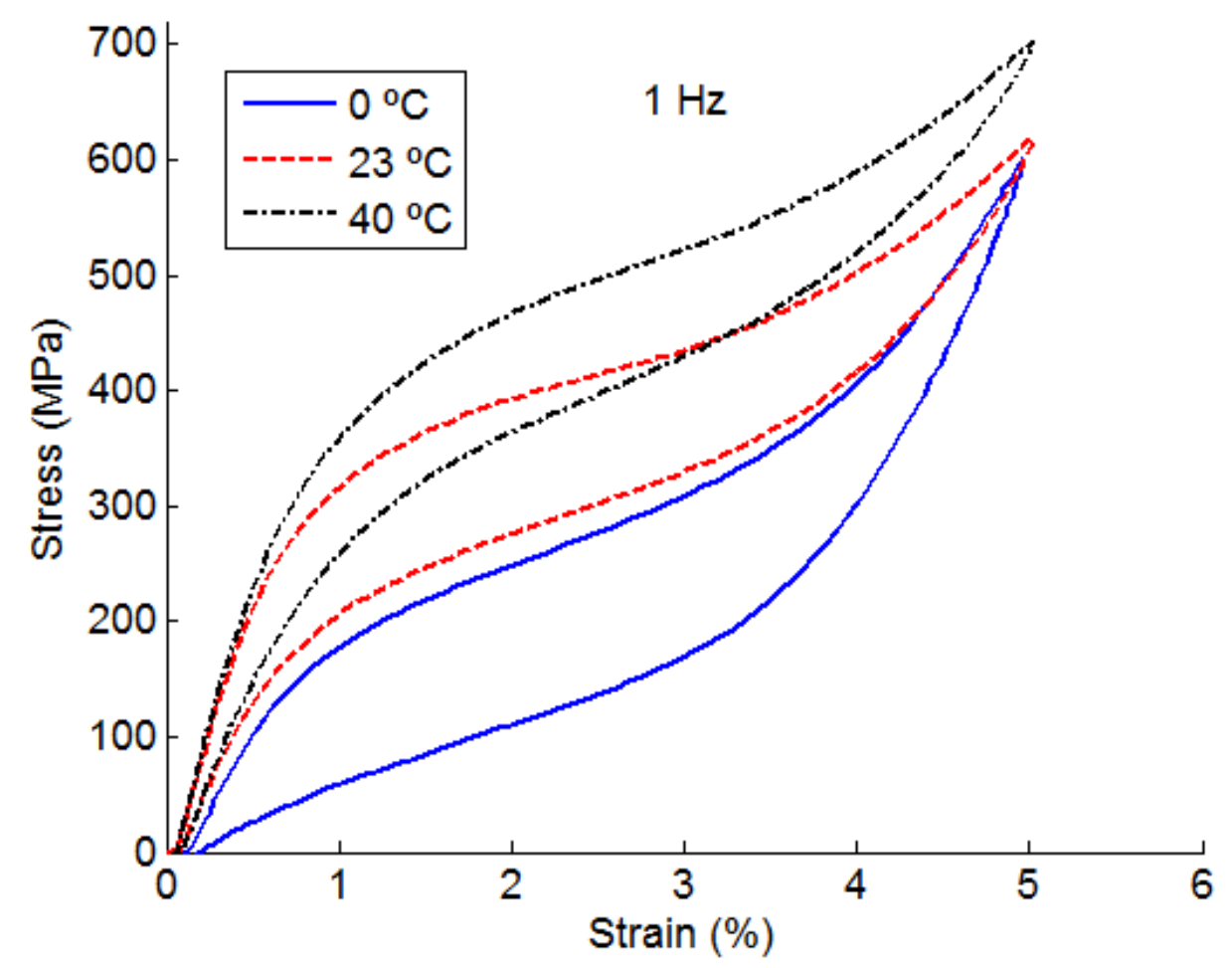

Figure 8-3 Stress - strain curves of SMAs at different temperatures 

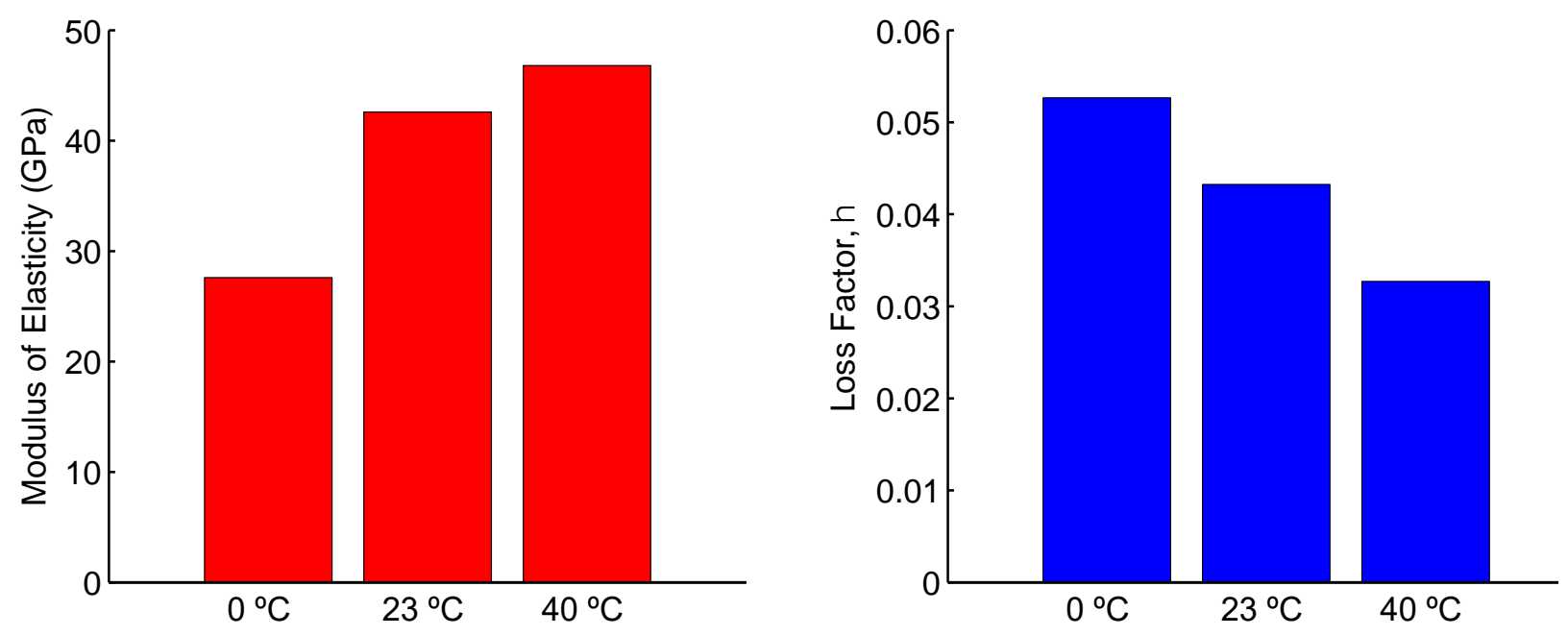

Figure 8-4 Variation of equivalent stiffness and loss factor for SMA with temperature

\subsection{Building Description and Numerical Modeling}

A nine-story steel moment resisting frame is selected for numerical investigations. The detail description of the selected steel frame and its design and modeling as SMRF and SVD frames are provided in Section 5.2.

The model of the superelastic viscous damper is developed in OpenSees as described in Section 5.2. The experimental test results at different temperatures on SMAs and HD butyl compound are used to develop model parameters for the SMA and elastomeric compound. The selected parameters for each temperature are shown in Table 8-1. 
Table 8-1 Model parameters for butyl rubber and SMAs for different temperatures

\begin{tabular}{|c|c|c|c|c|c|c|c|c|}
\hline \multirow[b]{2}{*}{ Temperature } & \multicolumn{4}{|c|}{ Butyl rubber } & \multicolumn{4}{|c|}{ SMA } \\
\hline & $\begin{array}{c}K \\
(\mathrm{kN} / \mathrm{mm})\end{array}$ & $\begin{array}{c}C \\
(\mathrm{kN}-\mathrm{s} / \mathrm{mm})\end{array}$ & $\alpha$ & $\begin{array}{c}k_{1} \\
(\mathrm{kN} / \mathrm{mm})\end{array}$ & $\begin{array}{c}k_{2} \\
(\mathrm{kN} / \mathrm{mm})\end{array}$ & $\gamma$ & $\begin{array}{r}F_{a} \\
(\mathrm{kN})\end{array}$ & $\beta$ \\
\hline $0^{\circ} \mathrm{C}$ & 2.15 & 0.92 & 0.8 & 2.03 & 1.07 & 1.4 & 46.26 & 1 \\
\hline $23^{\circ} \mathrm{C}$ & 1.43 & 1.00 & 0.8 & 3.12 & 0.969 & 1.2 & 71.17 & 0.5 \\
\hline $40^{\circ} \mathrm{C}$ & 1.22 & 1.01 & 0.8 & 3.59 & 1.24 & 1.2 & 81.99 & 0.389 \\
\hline
\end{tabular}

\subsection{Nonlinear Response History Analysis}

To evaluate effect of temperature on the performance of SVDs in mitigating the seismic response of steel frame structures, nonlinear response time history analyses are conducted for the steel frame with SVDs at different temperatures. The response of steel frame is evaluated under two different hazard levels: the design basis earthquake (DBE) seismic hazard level with a probability of exceedance of $10 \%$ in 50 years and the maximum considered earthquake (MCE) seismic hazard level with a probability of exceedance of $2 \%$ in 50 years. A total of 10 ground motion records that are also used in the FEMA P695 (FEMA, 2009) methodology is employed for nonlinear time history analyses.

Table 8-2 provides the characteristics of the selected ground motion records. The records are collectively scaled to selected hazard level, as defined in ASCE/SEI 7-05, such that the median spectral acceleration of the record set matches with those of the design spectra at the fundamental period of steel moment resisting frame. 
Table 8-2 Ground motion records used in analyses

\begin{tabular}{|c|c|c|c|c|c|c|c|}
\hline No. & Earthquake & Station Name & Component & $\begin{array}{l}\text { Magnitude } \\
\qquad\left(\mathrm{M}_{\mathrm{w}}\right)\end{array}$ & $\begin{array}{l}\text { Distance } \\
(\mathrm{km})\end{array}$ & $\begin{array}{l}\text { Peak Ground } \\
\text { Acceleration } \\
\text { (g) }\end{array}$ & $\begin{array}{l}\text { Peak } \\
\text { Ground } \\
\text { Velocity } \\
(\mathrm{cm} / \mathrm{s})\end{array}$ \\
\hline 1 & $\begin{array}{c}\text { Northridge } \\
\text { (1994) }\end{array}$ & $\begin{array}{l}\text { Beverly Hills- } \\
\text { Mulhol }\end{array}$ & MUL009 & 6.7 & 13.3 & 0.42 & 59 \\
\hline 2 & $\begin{array}{l}\text { Northridge } \\
\text { (1994) }\end{array}$ & $\begin{array}{l}\text { Beverly Hills- } \\
\text { Mulhol }\end{array}$ & MUL279 & 6.7 & 13.3 & 0.52 & 63 \\
\hline 3 & $\begin{array}{l}\text { Northridge } \\
\text { (1994) }\end{array}$ & $\begin{array}{c}\text { Canyon } \\
\text { Country-WLC }\end{array}$ & LOS000 & 6.7 & 26.5 & 0.41 & 43 \\
\hline 4 & $\begin{array}{l}\text { Northridge } \\
\text { (1994) }\end{array}$ & $\begin{array}{c}\text { Canyon } \\
\text { Country-WLC }\end{array}$ & LOS270 & 6.7 & 26.5 & 0.48 & 45 \\
\hline 5 & $\begin{array}{l}\text { Duzce, Turkey } \\
\text { (1999) }\end{array}$ & Bolu & BOL000 & 7.1 & 41.3 & 0.73 & 56 \\
\hline 6 & $\begin{array}{l}\text { Duzce, Turkey } \\
\text { (1999) }\end{array}$ & Bolu & BOL090 & 7.1 & 41.3 & 0.82 & 62 \\
\hline 7 & $\begin{array}{l}\text { Hector Mine } \\
\text { (1999) }\end{array}$ & Hector & HEC000 & 7.1 & 26.5 & 0.26 & 29 \\
\hline 8 & $\begin{array}{l}\text { Hector Mine } \\
\text { (1999) }\end{array}$ & Hector & HEC090 & 7.1 & 26.5 & 0.34 & 42 \\
\hline 9 & $\begin{array}{c}\text { Imperial Valley } \\
\text { (1979) }\end{array}$ & Delta & H-DLT262 & 6.5 & 33.7 & 0.24 & 26 \\
\hline 10 & $\begin{array}{c}\text { Imperial Valley } \\
(1979)\end{array}$ & Delta & H-DLT352 & 6.5 & 33.7 & 0.35 & 33 \\
\hline
\end{tabular}

Structural responses of the uncontrolled building and the building with the installed hybrid dampers are computed under each ground motion record at two seismic hazard levels. The distribution of the peak inter-story drift and peak floor acceleration over the height of the building are computed for the steel frame with the installed SVDs at different temperatures. Median response of ten records for each temperature at the DBE level and MCE level is provided in Figures 8-5 and 8-6.

It can be seen from Figures 8-5 and 8-6 that the SVD system has the lowest inter-story drift and acceleration response values at both the DBE and MCE levels when the temperature is $0^{\circ} \mathrm{C}$. This can be attributed to the fact that the damping ratio for both butyl rubber and SMAs increases with a decrease in temperature. Also, the stiffness of the SMAs considerably decreases at $0^{\circ} \mathrm{C}$, 
which reduces overall stiffness of the SVDs device at lower temperatures and lead to a reduction in acceleration response. Since the energy dissipation capacity of both butyl rubber and SMAs is lower at $40^{\circ} \mathrm{C}$ compared to $23^{\circ} \mathrm{C}$, the peak story drifts has the largest values at $40^{\circ} \mathrm{C}$ for the frame with SVDs. Due to the increase in SMA forces at $40^{\circ} \mathrm{C}$, the peak acceleration response is slightly higher at $40^{\circ} \mathrm{C}$ compared to that at $23^{\circ} \mathrm{C}$. When the temperature drops from $23^{\circ} \mathrm{C}$ to $0^{\circ} \mathrm{C}$, the median story drift at each floor changes between $+1 \%$ and $-18 \%$, and the median floor accelerations experience a decrease between a minimum of $0.4 \%$ and a maximum of $19 \%$ under DBE and MCE level ground motions. On the other hand, when the temperature increases from $23^{\circ} \mathrm{C}$ to $40^{\circ} \mathrm{C}$, the median story drift at each floor increases between $0.1 \%$ and $7 \%$, and the median floor accelerations experience a varies between $+2 \%$ and $+11 \%$ under DBE and MCE level ground motions.
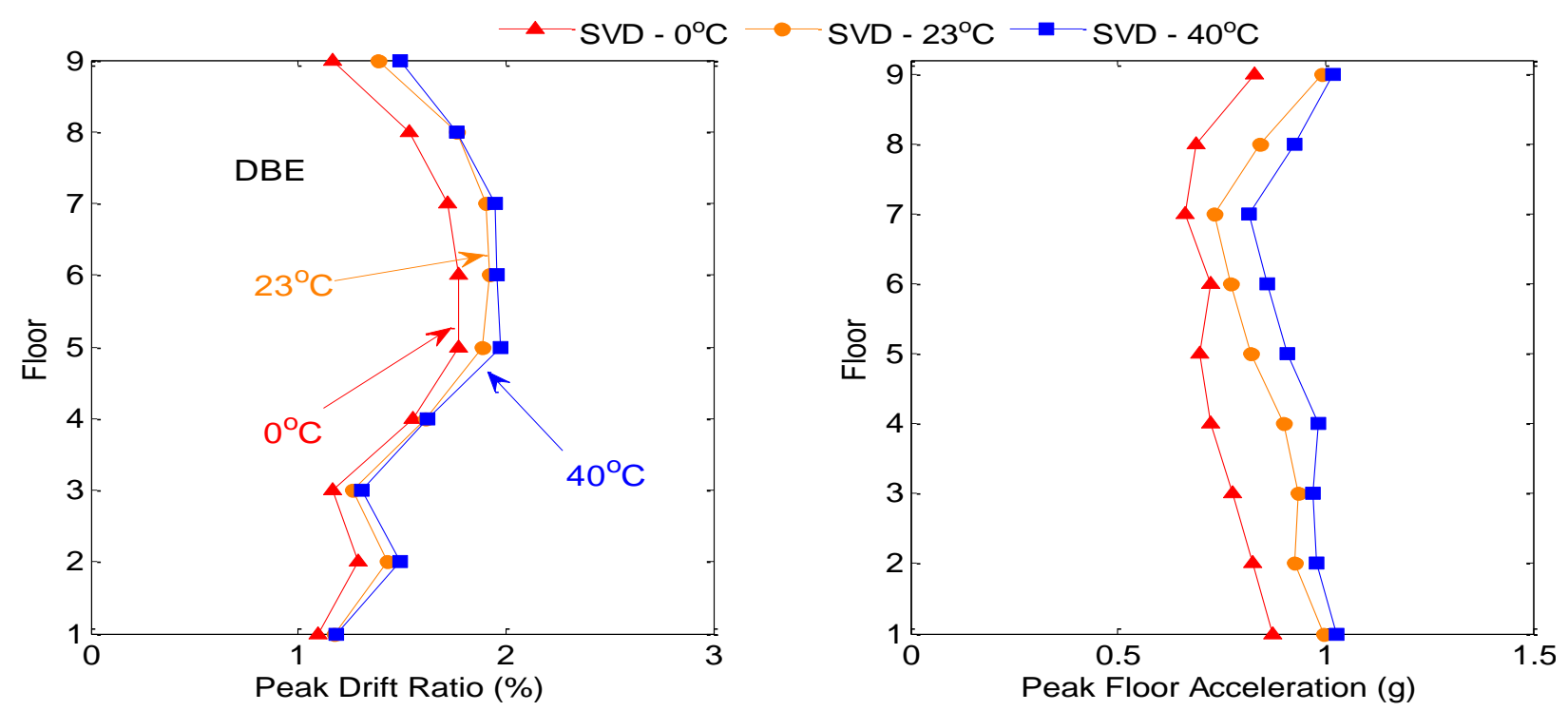

Figure 8-5 Envelopes for median of peak response quantities for SVD systems at different temperatures at DBE level hazard 

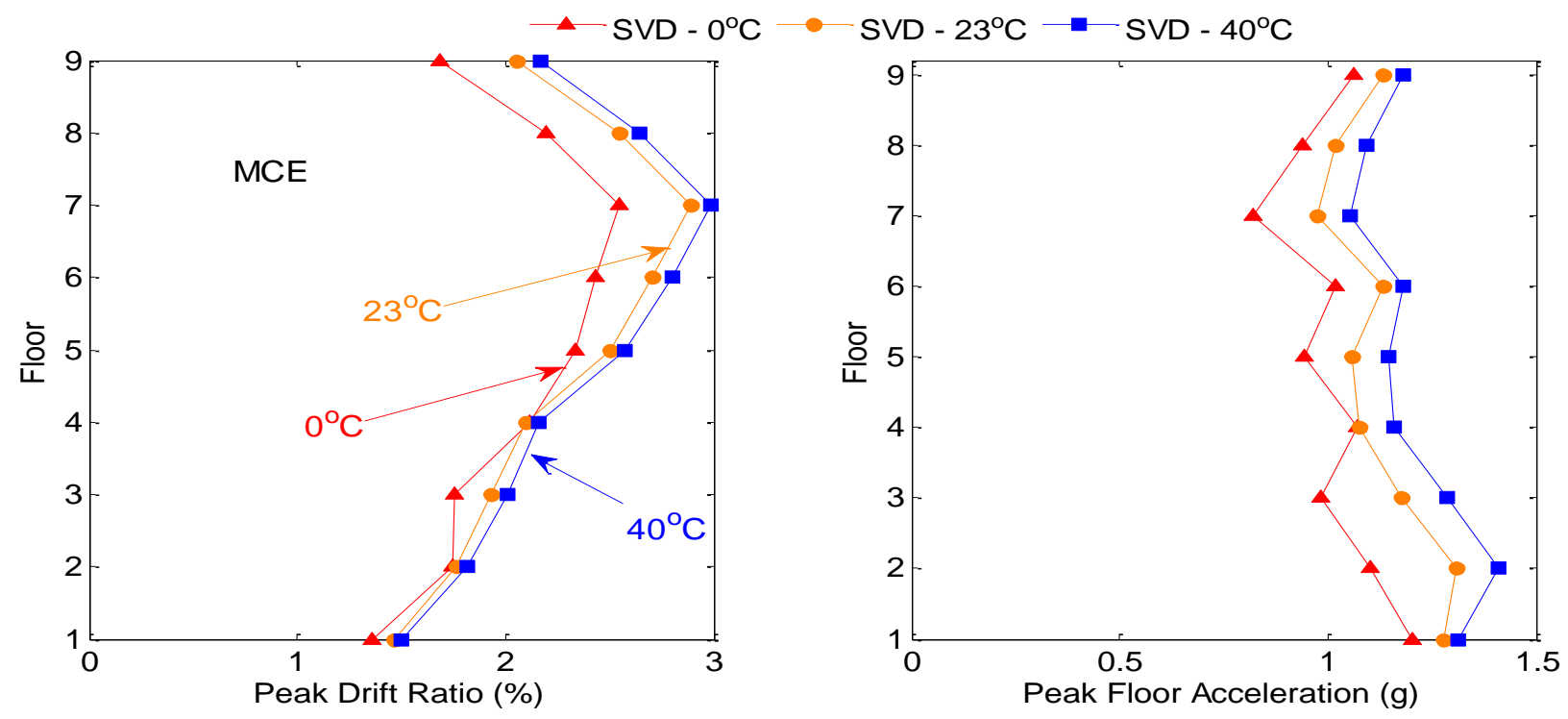

Figure 8-6 Envelopes for median of peak response quantities for SVD systems at different temperatures at MCE level hazard

In order to further evaluate the findings discussed above, Figure 8-7 illustrates the forcedisplacement curves of all SVDs installed to the $6^{\text {th }}$ floor at different temperatures. A larger hysteresis loop, which indicates more energy dissipation, is observed at $0^{\circ} \mathrm{C}$. Due to higher damping at $0^{\circ} \mathrm{C}$, peak story drifts attained their minimal values at that temperature. In addition, it can be seen that higher forces with larger stiffness are developed at $40^{\circ} \mathrm{C}$, which caused higher acceleration responses at high temperature. 

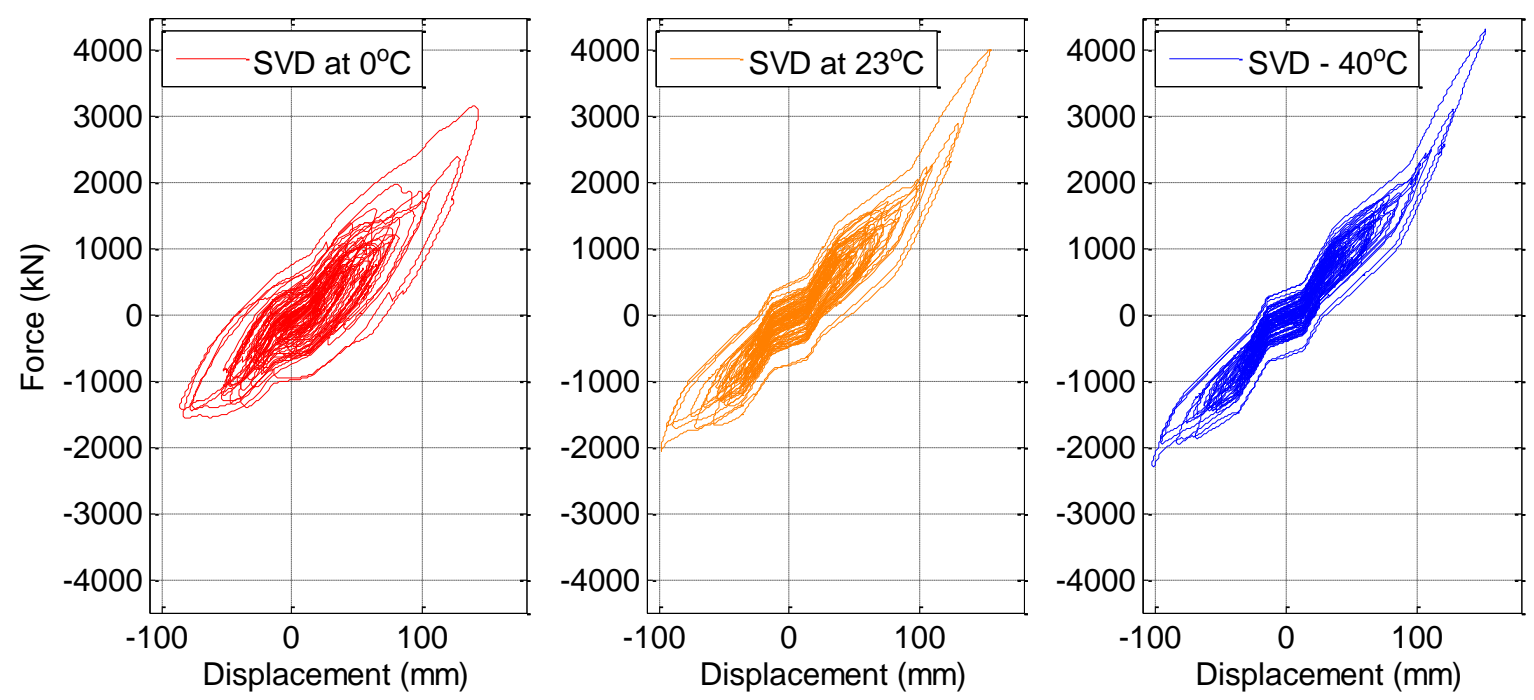

Figure 8-7 Force-displacement curves of SVDs at different temperatures under Imperial Valley earthquake

\subsection{Closure}

In this section, the seismic performance of a nine-story steel moment resisting frame equipped with SVDs is investigated under different temperatures. First, the effects of temperature on both butyl rubber and SMAs are characterized through experimental testing. Then, the ninestory steel moment resisting frame is designed with SVDs. Numerical models for both nine-story building and frame with the SVD device are generated in a finite element program. The parameters of the SVD model are adjusted for three temperatures: $0^{\circ} \mathrm{C}, 23^{\circ} \mathrm{C}$ and $40^{\circ} \mathrm{C}$. The peak response quantities of the building are determined through the nonlinear response history analyses at each temperature. Results shows that both the peak story drift and peak floor acceleration have their minimal values at $0^{\circ} \mathrm{C}$, while larger responses are observed at $40^{\circ} \mathrm{C}$. Nevertheless, it is shown that designing structures with SVDs considering the room temperature material properties of SVDs will underestimate both the peak drift and acceleration response at various temperatures only about $10 \%$. 


\section{SEISMIC LOSS ASSESSMENT OF STEEL FRAME BUILDINGS WITH SVDS}

\subsection{Introduction}

In this section, the seismic loss assessment of steel building installed with superelastic viscous damper is conducted, by employing the performance-based seismic assessment methodology illustrated in the FEMA P-58 (FEMA, 2012). Incremental dynamic analyses on SMRF and SVD frames are carried out to assess the collapse capacity of structural systems. Then, nonlinear time history analyses are conducted for both structural systems at different seismic hazard levels. The seismic loss evaluation is performed by employing the Performance Assessment Calculation Tool (PACT) software. The building information, performance groups, structural analysis results, and seismic hazard data are defined in the PACT loss analysis tool. The structural damage consequences of the SMRF and SVD frames are compared in terms of the expected repair cost and repair time of structural systems under multiple seismic hazard levels.

\subsection{Building Description and Modeling}

A nine-story steel moment resisting frame is selected for numerical investigations. The detailed description of the selected steel frame, and its design and modeling as the SMRF and SVD frames, are provided in Section 5.2.

\subsection{Seismic Loss Assessment Methodology}

The seismic loss assessment of steel frame buildings with and without installed SVDs is evaluated based on the FEMA P-58 methodology, which describes five fundamental steps for determining the loss assessment as shown in Figure 9-1. First, the building performance model is described through assigning data for structural components, nonstructural components, and their exposure to earthquake hazards. The vulnerable building components are divided into fragility groups, which are recognized by sets of similar components indicating the same potential damage characteristics in terms of vulnerability and consequences, and performance groups, which are subsets of the fragility groups experiencing the same earthquake demands. Next, the earthquake 
hazard is defined to quantify the intensity measure of ground motions and the site-specific probability for the corresponding intensity measure.

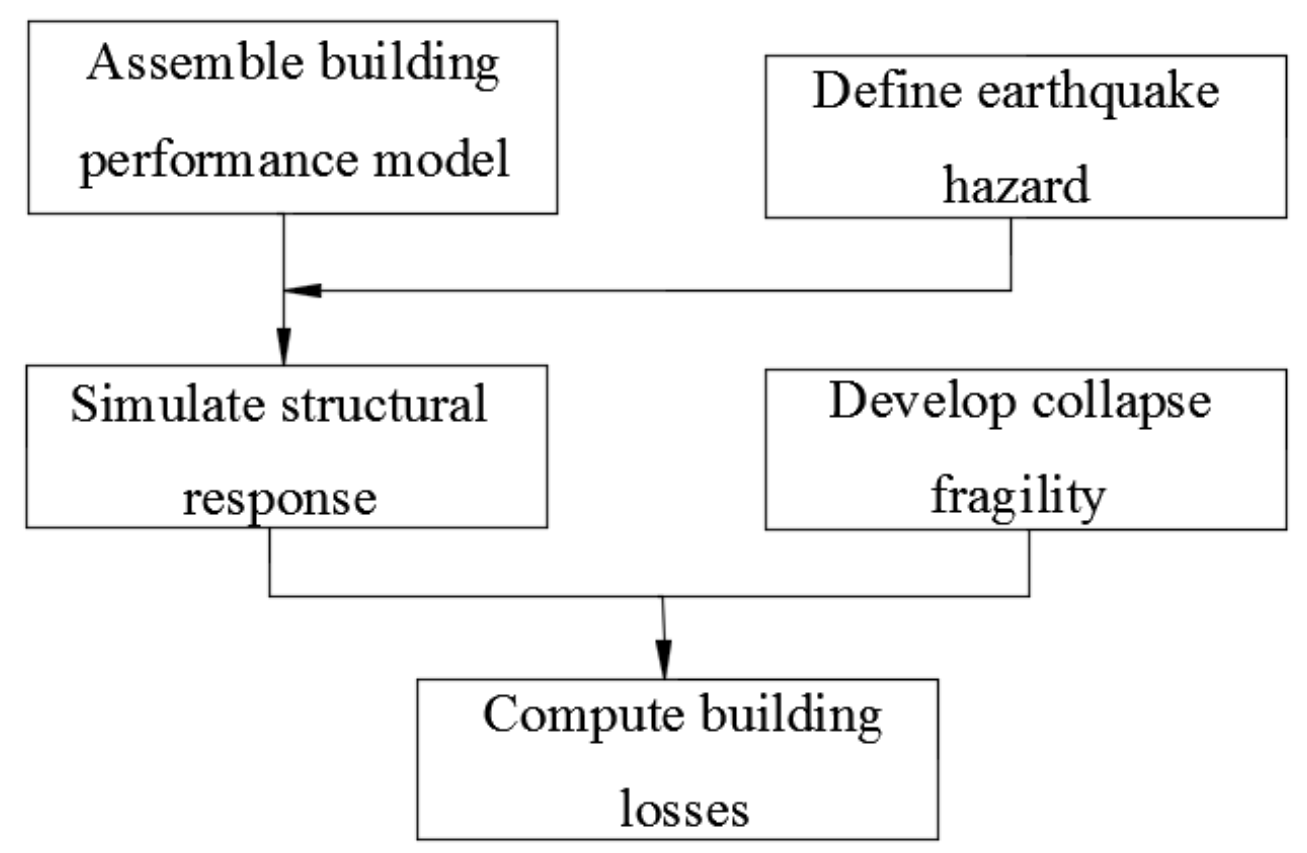

Figure 9-1 FEMA P-58 Procedure for seismic loss assessment

Then, nonlinear structural analysis procedure is used to determine the structural demand quantities of building structure, which include peak inter-story drift ratio, peak floor acceleration, and residual drift ratio. These demand quantities are associated with both structural and nonstructural damage. After predicting the structural response quantifications at different hazard levels for both SMRF and SVD frames, collapse fragility functions are developed to assess the potential casualties that can occur by the partial or total collapse of building structures. Based on the structural analysis and engineering judgement, the probability of incurring structural collapse is estimated for establishing collapse fragilities with the possible modes of structural collapse.

Finally, to calculate building losses, PACT tool utilizes a Monte Carlo simulation procedure to determine the potential distributions of economic losses, considering the many uncertainties inherent in a seismic performance evaluation. The Monte Carlo procedure is an 
iterative process to calculate building performance considering a large number of realizations. In this study, 200 realizations are used to evaluate building performance. Since structural analysis results provide median response values and dispersion to represent modeling dispersion and scenario uncertainty, the median value matrix and correlation matrix together with the dispersions are employed to generate potential simulated response states in the PACT software. Each simulated response state is recognized with one realization, which represents one potential performance of the building's earthquake response, corresponding to an intensity measure. Figure 9-2 represents the performance calculation process in each realization in order to compute losses for each realization.

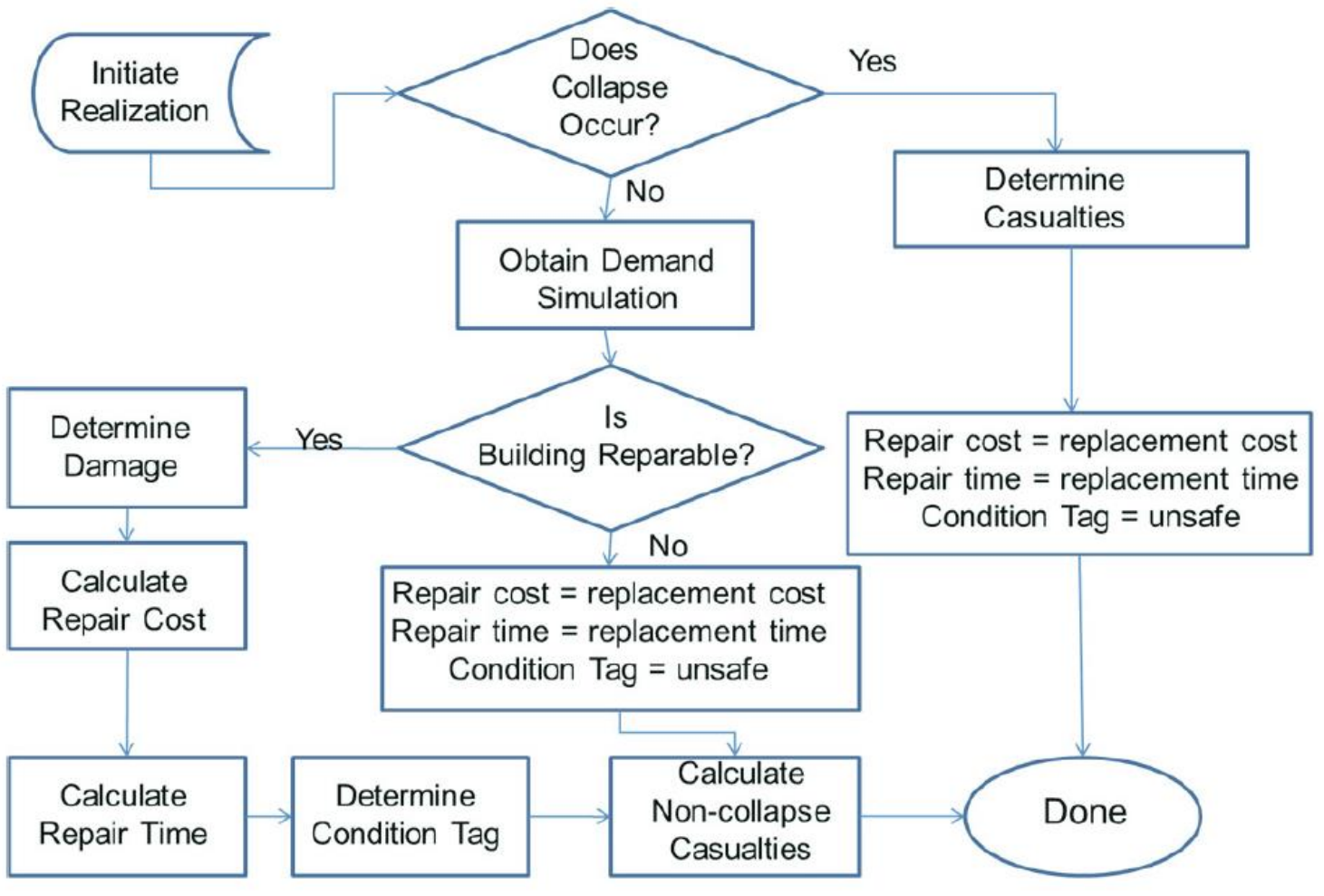

Figure 9-2 Performance calculation process in each realization (FEMA P-58-1) 


\subsection{Seismic Hazard Curve}

The seismic hazard curve for a nine-story steel frame located in Seattle, Washington, is generated for the site class D. Figure 9-3 shows the seismic hazard curve of the nine-story frame at fundamental period of 2.44 seconds, expressing the relationship between the annual frequency of exceeding earthquake intensity and the corresponding spectral acceleration. Typically, earthquake intensity is measured as the spectral acceleration at the first mode period of a building. To assign the hazard curve data to the selected intensity measures in the PACT software, the hazard curve data at six intensity measures are determined, indicating hazard levels ranging from $20 \%$ probability of exceedance (PE) in 50 years to two times the hazard level at maximum considered earthquake (MCE). Figure 9-4 and Table 9-1 show the seismic hazard curve plot at the selected intensity measure, and the corresponding hazard data at six intensity measures.

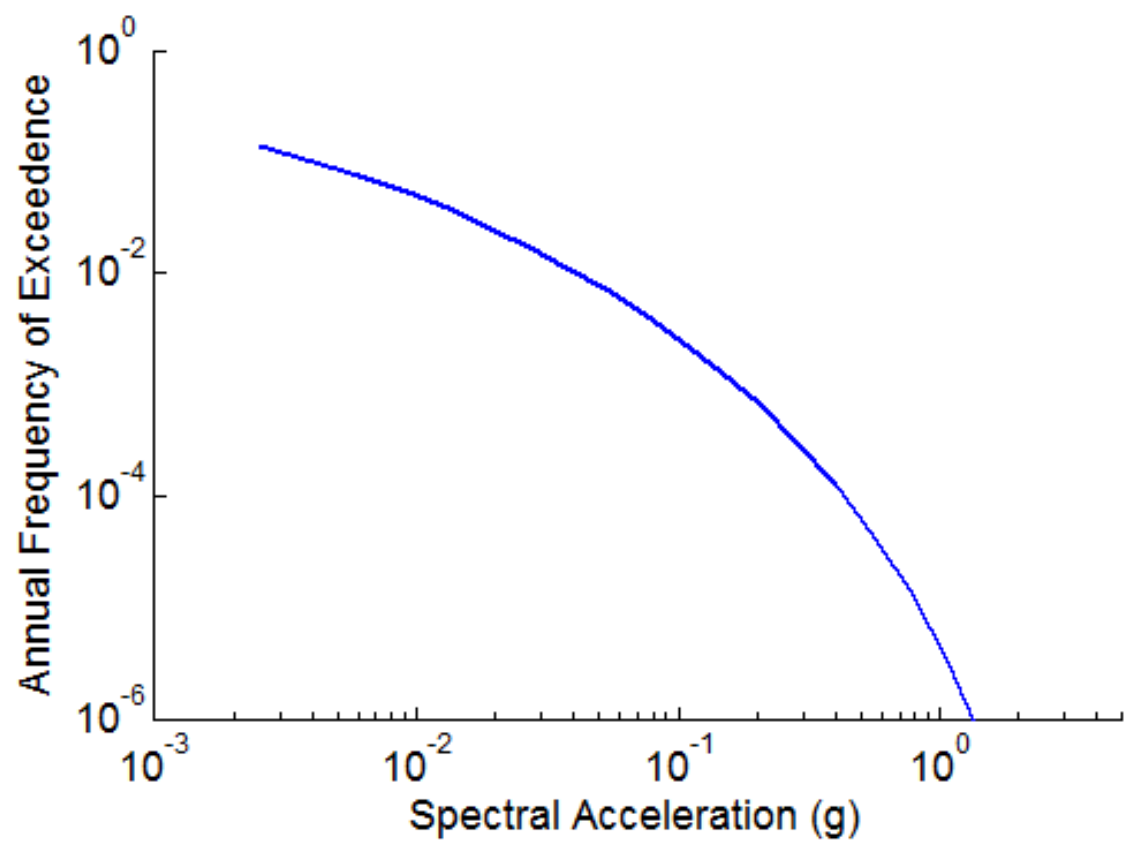

Figure 9-3 Seismic hazard curve for a nine-story steel frame located in Seattle 


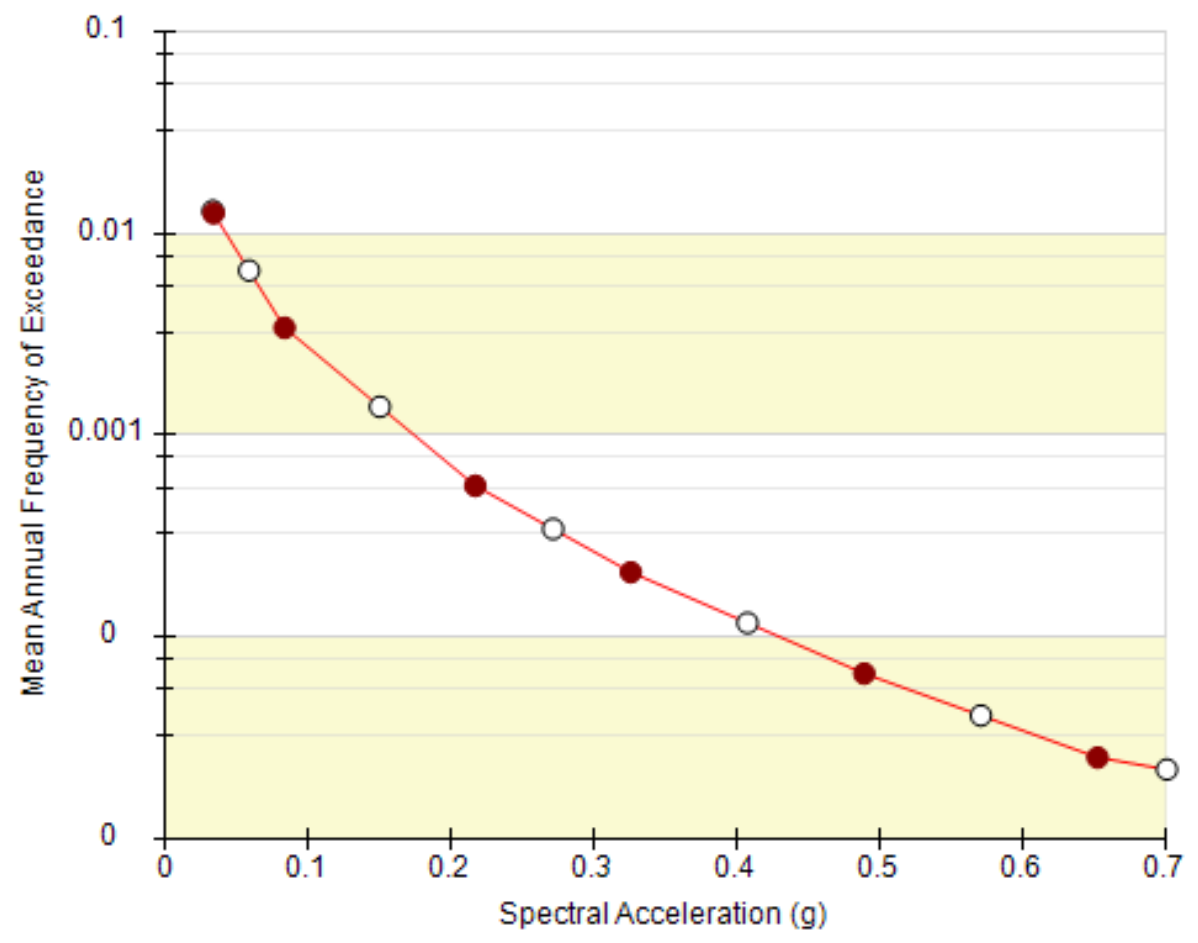

Figure 9-4 Hazard curve at selected intensity measure used in PACT

Table 9-1 The hazard curve data at the selected intensity level

\begin{tabular}{c|l|l|}
\multicolumn{1}{c}{} & \multicolumn{1}{c}{ Sa (g) } & \multicolumn{1}{l}{ MAFE } \\
\cline { 2 - 3 } Sa Min Point & 0.034 & 0.013 \\
\cline { 2 - 3 } \multicolumn{1}{c}{ Sa Max Point } & 0.7 & $2.2 \mathrm{E}-05$ \\
\hline RunName & Sa & MAFE \\
\hline Intensity 1 & 0.0345348 & 0.012619632 \\
\hline Intensity 2 & 0.084079206 & 0.00341704 \\
\hline Intensity 3 & 0.2172 & 0.000562091 \\
\hline Intensity 4 & 0.3258 & 0.000209266 \\
\hline Intensity 5 & 0.4887 & $6.57667 \mathrm{E}-05$ \\
\hline Intensity 6 & 0.6516 & $2.52059 \mathrm{E}-05$ \\
\hline
\end{tabular}




\subsection{Nonlinear Response History Analysis}

Nonlinear response history analyses are performed for the steel frames designed as SMRF and SVD frames, under 10 far-field ground motion records described in Section 8 and detailed in Table 8-2. The structural responses of the steel frame are evaluated under six different hazard levels. The selected hazard levels are: seismic hazard level with a $20 \%$ probability of exceedance (PE) in 50 years; frequent level of earthquake (FOE); the design basis earthquake (DBE); the maximum considered earthquake (MCE); 1.5 $\times \mathrm{MCE}$; and 2.0×MCE. The distinct scale factor at the fundamental period of the SMRF is selected for each record to represent the selected hazard level. In order to compare the seismic loss assessment, both the SMRF and SVD frames are scaled by the same scale factor, so that identical seismic hazards are assigned for both frame systems.

The structural response quantities for both SMRF and SVD frames are computed at each hazard level. Peak demands are expressed in terms of the distribution of the peak inter-story drift ratio, peak floor acceleration, and the peak residual drift ratio over the height of the nine-story steel buildings. These quantities for each hazard level are assigned in the PACT software to evaluate the performance of both frame systems.

In the PACT methodology, the potential distribution of building damage, to structural and nonstructural components and building contents, are determined from the results of the structural analysis with the information on the building's configurations. Since the damage prediction is uncertain even for a specific demand, component or frame-system specific fragility functions are used to characterize damage for the demand identified from the structural analysis. The fragility curves are used for the assessment of the damage at the given demand, as mentioned in the subsequent section.

\subsection{Evaluation of Collapse Fragility}

The fragility curve provides the probability of incurring damage at the given demand, where demand is measured by any useful response quantity, such as story drift and floor acceleration in this study. Most earthquake casualties are caused by the partial or complete collapse of the building, and the PACT methodology calculates these casualties relating damage associated to the specific hazard level. However, damage to each structural and nonstructural component in 
a building may be highly uncertain, due to the unique probability of sustaining damage during an earthquake. Hence, defining fragility function seems imperative to assess seismic losses. In the PACT methodology, the collapse fragility function indicates the probability of building collapse as a function of the spectral acceleration at the first mode period of the building. The fragility functions are developed in the PACT software using the log-normal distributions defined by a median value and dispersion.

Here, incremental dynamic analysis (IDA) is adopted to determine the collapse fragility (FEMA, 2009). The IDA results presented in Section 5 are used to develop collapse fragility curves for the SMRF and SVD frames. In this study, the intensity measure is selected as the 5\% spectral acceleration at the fundamental period of the SMRF. The collapse fragilities are $0.63 \mathrm{~g}$ and $0.87 \mathrm{~g}$ for the SMRF and SVD frames, considering a potential collapse limit state corresponding to 5\% inter-story drift ratio, as recommended in previous studies (Hsiao et al., 2013). Figure 9-5 displays the fragility curves of the SMRF and SVD frames, indicating the relationship between the spectral acceleration $\mathrm{S} a\left(\mathrm{~T}_{1}, 5 \%\right)$ and the probability of collapse. It can be seen that a frame upgraded with SVDs indicates higher collapse capacity ( $\mathrm{S}_{C T}=0.87 \mathrm{~g}$ ) compared to SMRF ( $\mathrm{S}_{C T}=0.63 \mathrm{~g}$ ). The SVD frame exhibits significantly better performance, illustrated by the fragility curve's shift to the right of the SMRF. Hence, the installation of SVD dampers in steel frames results in higher collapse resistance, compared to the SMRF. 


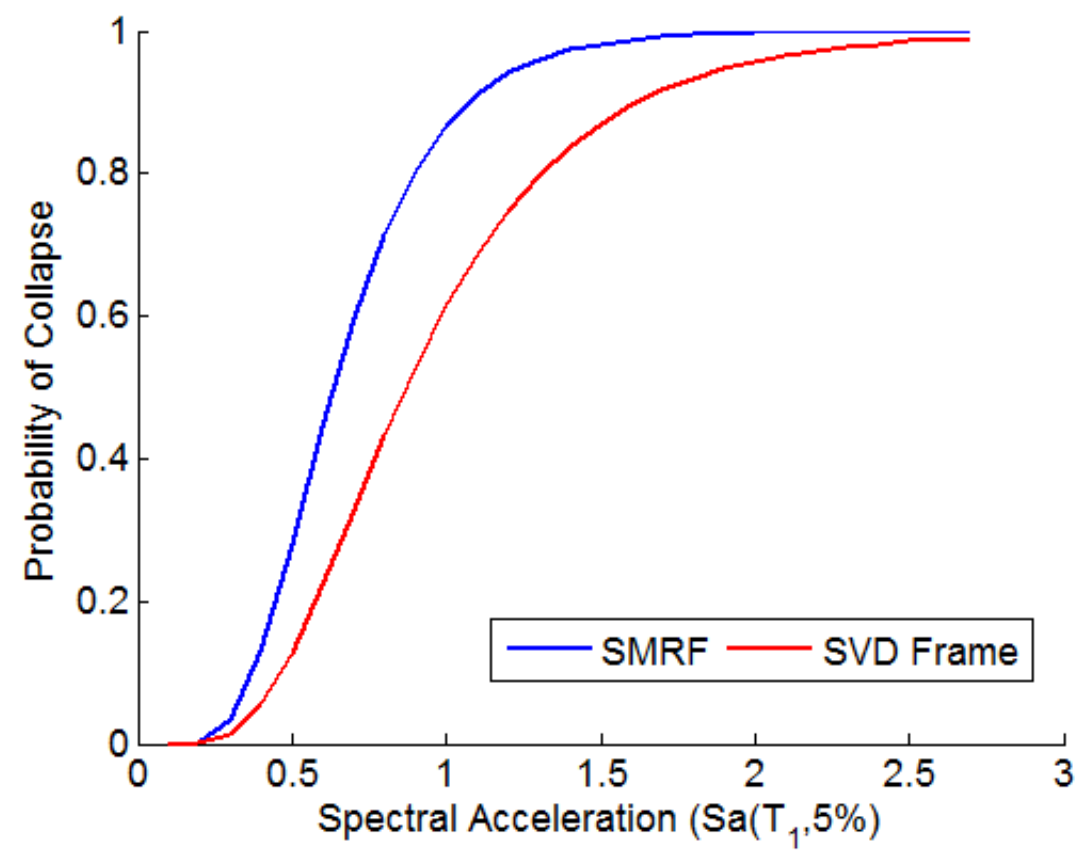

Figure 9-5 Collapse fragility curves for the SMRF and SVD frames

\subsection{Performance Assessment Results}

\subsubsection{PACT Results for Repair Cost and Repair Time}

The PACT tool is used to conduct the loss estimation analysis for both SMRF and SVD frames under different hazard levels in order to assess the repair cost, repair time, story-wise distribution of repair time, and the disaggregation of building loss.

The total replacement costs of SMRF and SVD frames are calculated as $\$ 37,500,000$ and $\$ 38,150,000$ for 2011 national average cost considering unit price of $\$ 180$ per square foot for the construction cost of office buildings in 2016, \$4000 for each damper, and a date cost multiplier of 1.08. Next, the regional cost multiplier is taken as 0.97 for Seattle, Washington, since the PACT software was developed for the Los Angeles location, considering the cost of structures in 2011. The core and shell replacement cost is taken as $40 \%$ of the total replacement cost. Similarly, the replacement times are selected as 200 days and 215 days for the SMRF and SVD frames, respectively. 
Figure 9-6 illustrates the comparative repair total costs and times for both SMRF and SVD frames under different hazard levels. It can be observed that the steel frame building installed with SVDs improves building performance by reducing the repair cost at different hazard levels up to twice the MCE hazard levels. For example, the building frame installed with SVDs has reduced the repair cost by $44.8 \%$ and $42.3 \%$ at DBE level and MCE level of seismic hazard, compared with the repair cost of the SMRF at the corresponding hazard level. Similarly, the repair time required for the SVD frame is reduced by $50.8 \%$ and $34.0 \%$ at DBE level and MCE level, compared to the repair time for the SMRF frame. However, when the hazard level is high, the repair times for the SMRF and SVD frames approximate each other, with the replacement time of 200 days for the SMRF and 215 days for the SVD frame. Hence, the installation of SVD dampers in a steel frame improves performance by reducing both repair cost and repair time at different hazard levels of earthquake events.
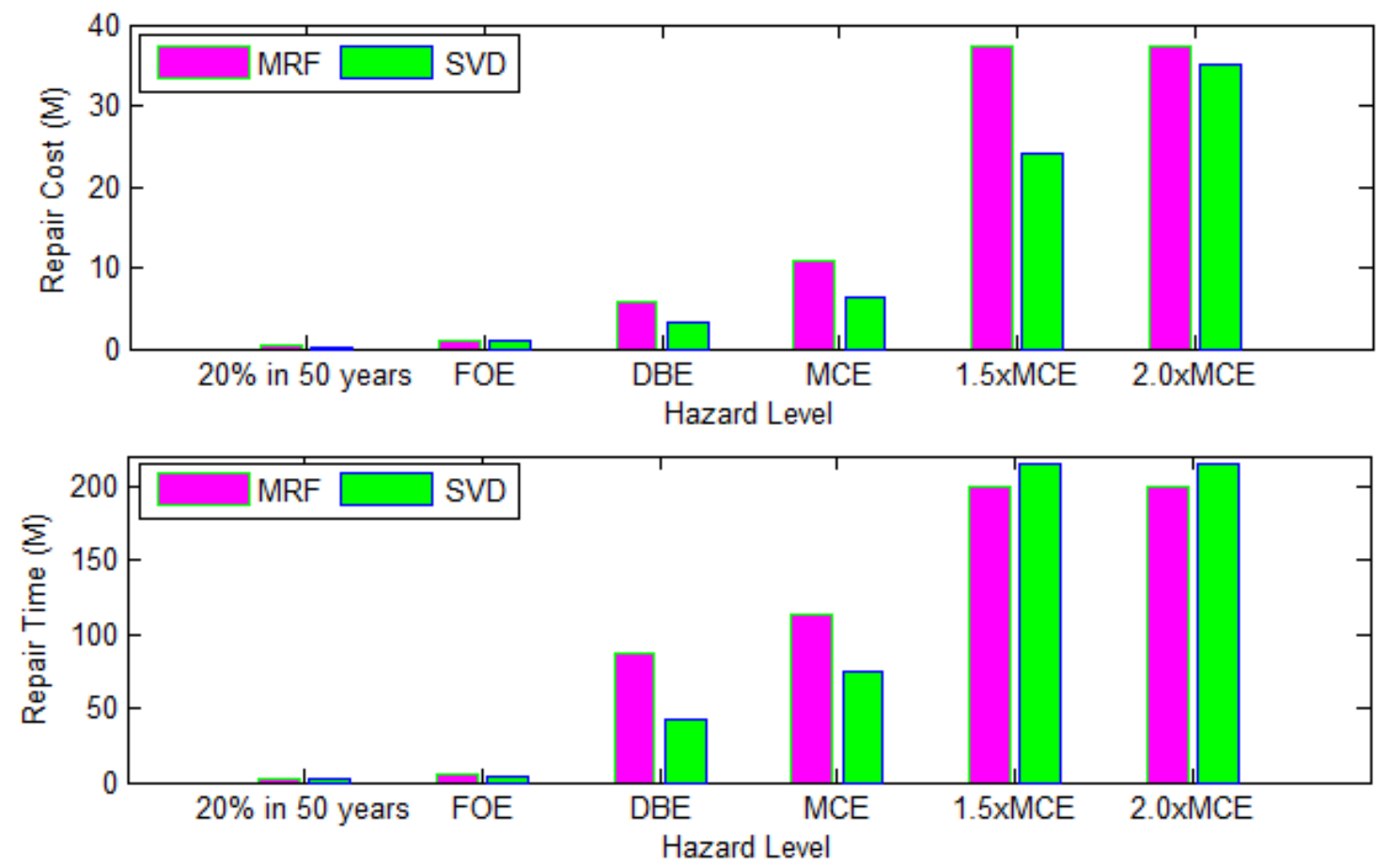

Figure 9-6 Comparative repair costs and repair times for SMRF and SVD frames at different hazard levels 
Figures 9-7 and 9-8 illustrates the repair costs of structural and nonstructural components for both SMRF and SVD frames at DBE level and MCE level of seismic hazards, respectively. The figures provide a weighted average of realizations for different performance groups to both SMRF and SVD frames. It can be seen that the structural component for the SVD frame indicates a lower replacement cost at DBE level compared with the SMRF. Furthermore, the total repair cost for both the structural and nonstructural component for the SVD frame is lower compared with the SMRF, at DBE level of seismic hazard. At MCE level of seismic hazard, the SVD frame shows significant improvement in performance by reducing the repair cost for both structural and nonstructural components, compared to the repair cost of the SMRF. Although the repair costs for structural components for both the SMRF and SVD frames are higher compared with the repair cost of the nonstructural components, the overall performance of the SVD frame is better in lowering the total repair cost by $44.8 \%$ and $42.3 \%$ at the DBE and MCE levels, compared with the repair cost of the SMRF at corresponding hazard levels.

Figures 9-9 and 9-10 present the story-wise distribution of repair times required for the SMRF and SVD frames at the DBE and MCE levels of seismic hazard, highlighting the required repair times for both the structural and nonstructural components at different stories. It can be seen that the SVD frame requires lower repair time at different stories, compared to the repair time for the SMRF frame at both DBE and MCE hazard levels. Although structural components in both the SMRF and SVD frames require more repair time compared to the repair time for the nonstructural components, the repair time required for the SVD frame is significantly reduced at different stories for both the DBE and MCE hazard levels. At DBE level, as shown in Figure 9-9 the SMRF demands more repair time at Floors 1 and 4, while the SVD frame needs more repair time at Floors 3 and 9. At MCE level, as shown in Figure 9-10, the SMRF demands more repair time at Floors 4 and 5, while the SVD frame requires more repair time at Floors 3 and 6, compared to the repair time required for the other floors. Overall, the steel frame installed with SVDs saves repair time at the DBE and MCE hazard levels, compared to the repair time required for the SMRF frame. 


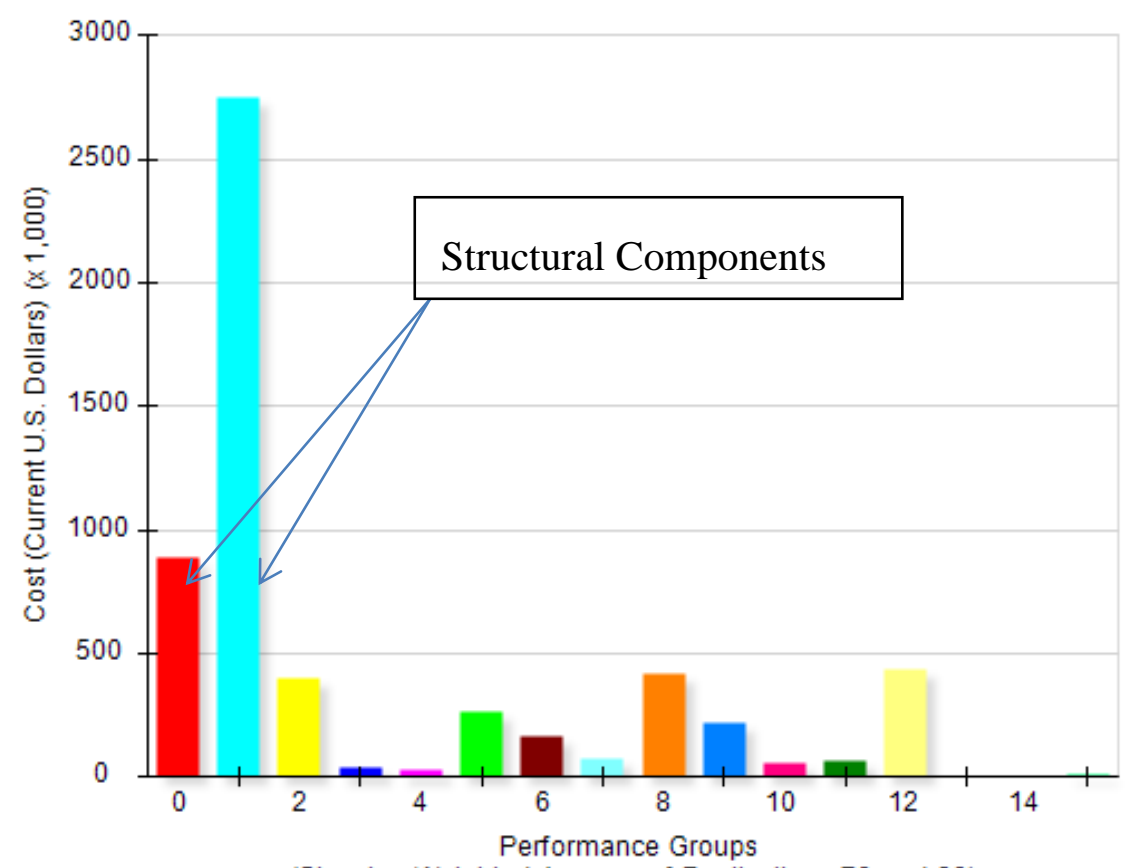

(a)

(Showing Weighted Average of Realizations 76 and 28)

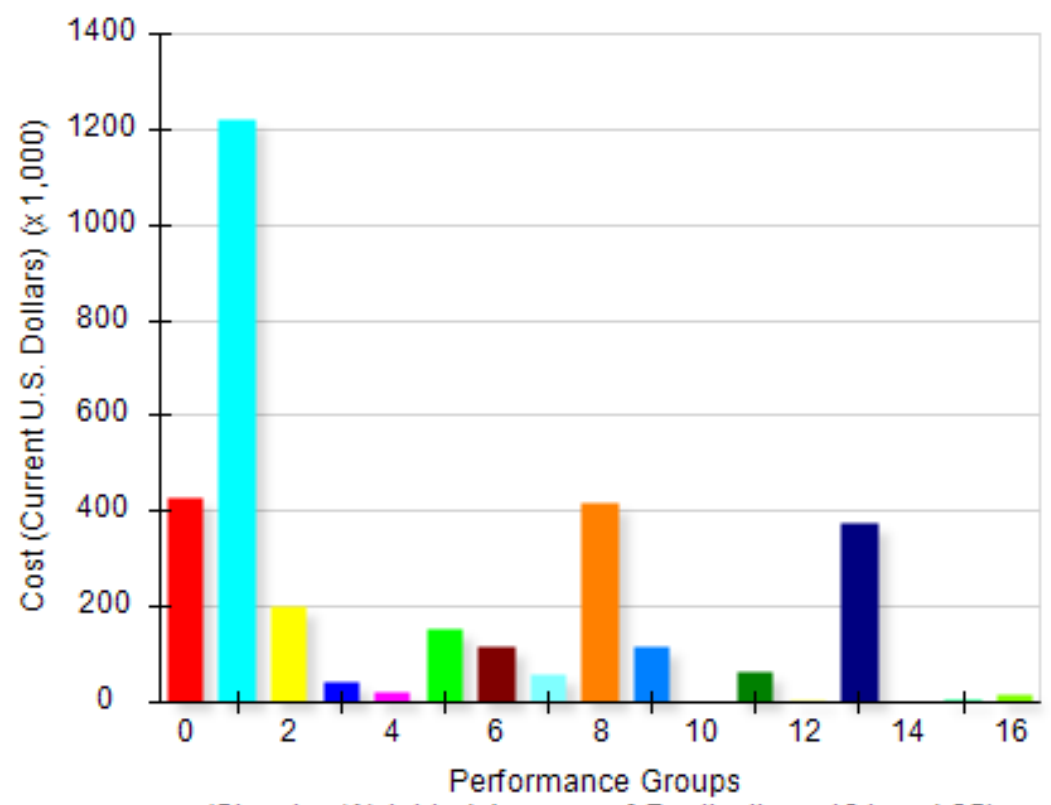

(b)

(Showing Weighted Average of Realizations 161 and 25)

Figure 9-7 Repair cost at DBE level: (a) SMRF and (b) SVD frames 


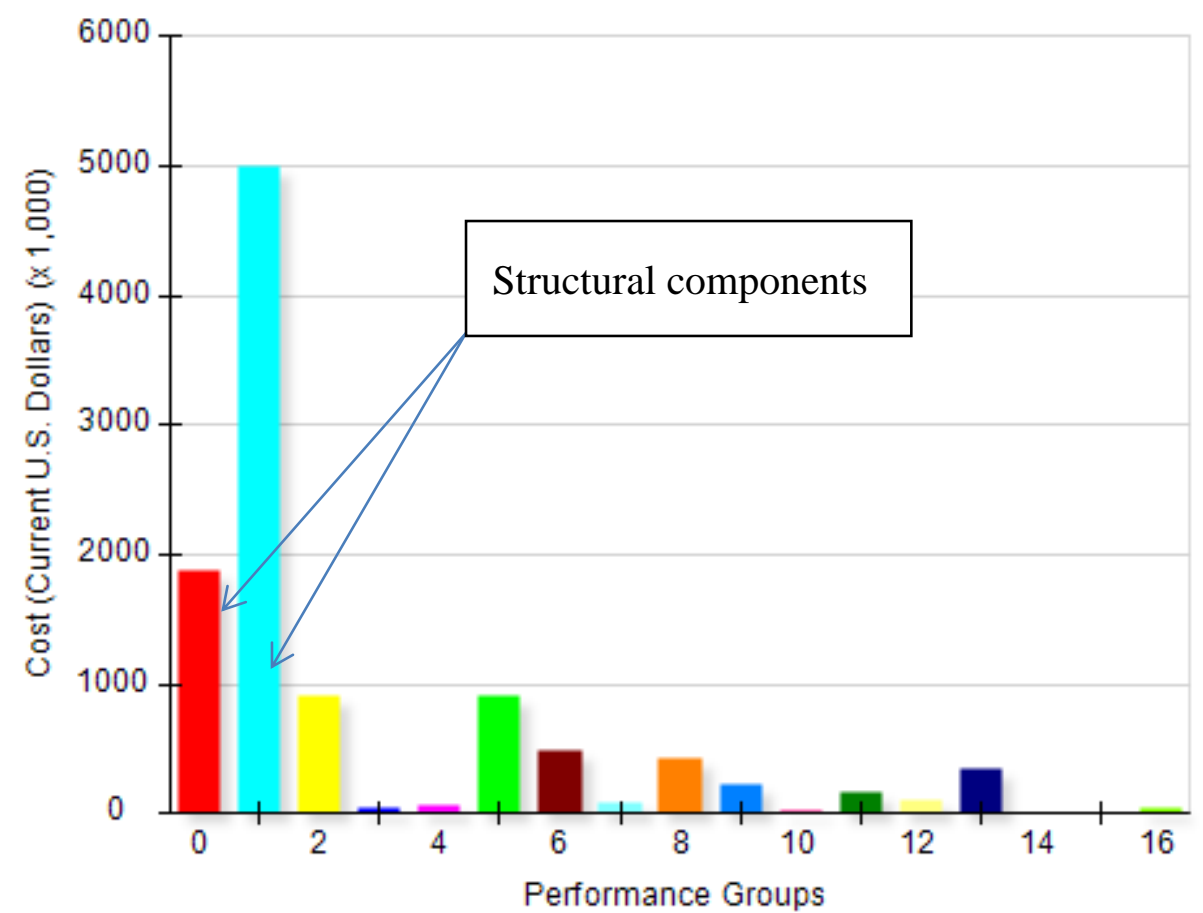

(a)

(Showing Weighted Average of Realizations 34 and 134)

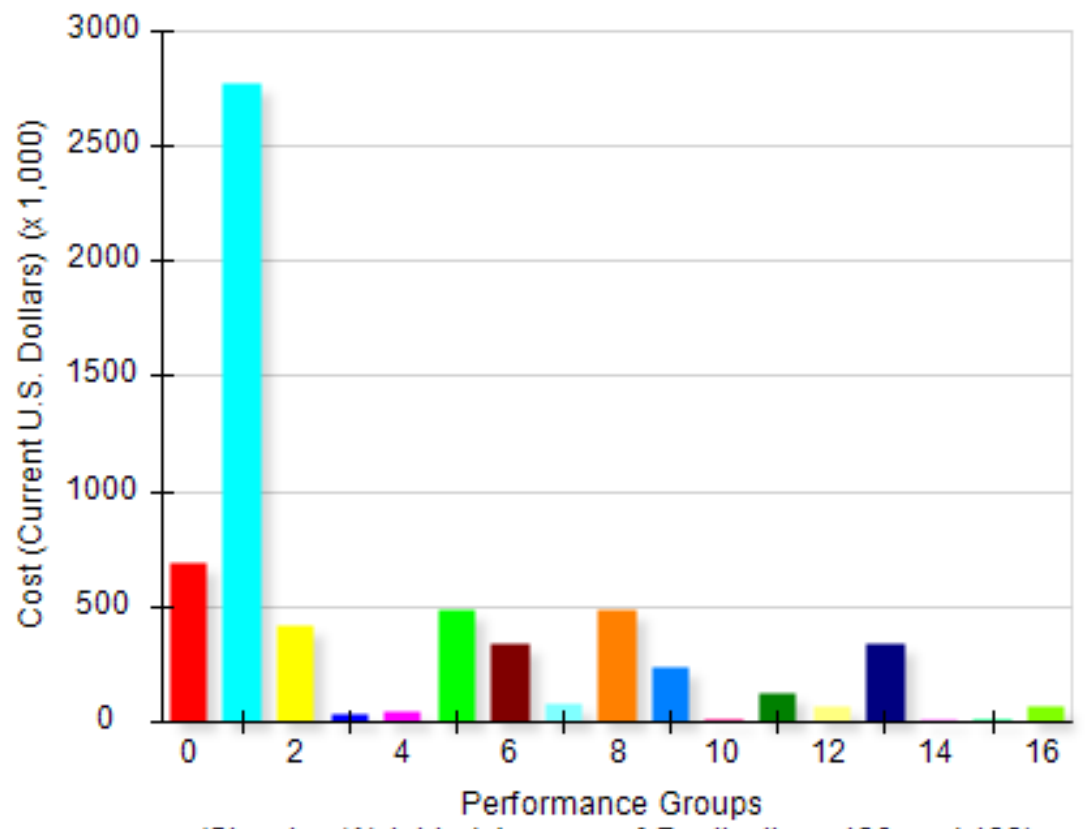

(b)

(Showing Weighted Average of Realizations 130 and 122)

Figure 9-8 Repair cost at MCE level: (a) SMRF and (b) SVD frames 


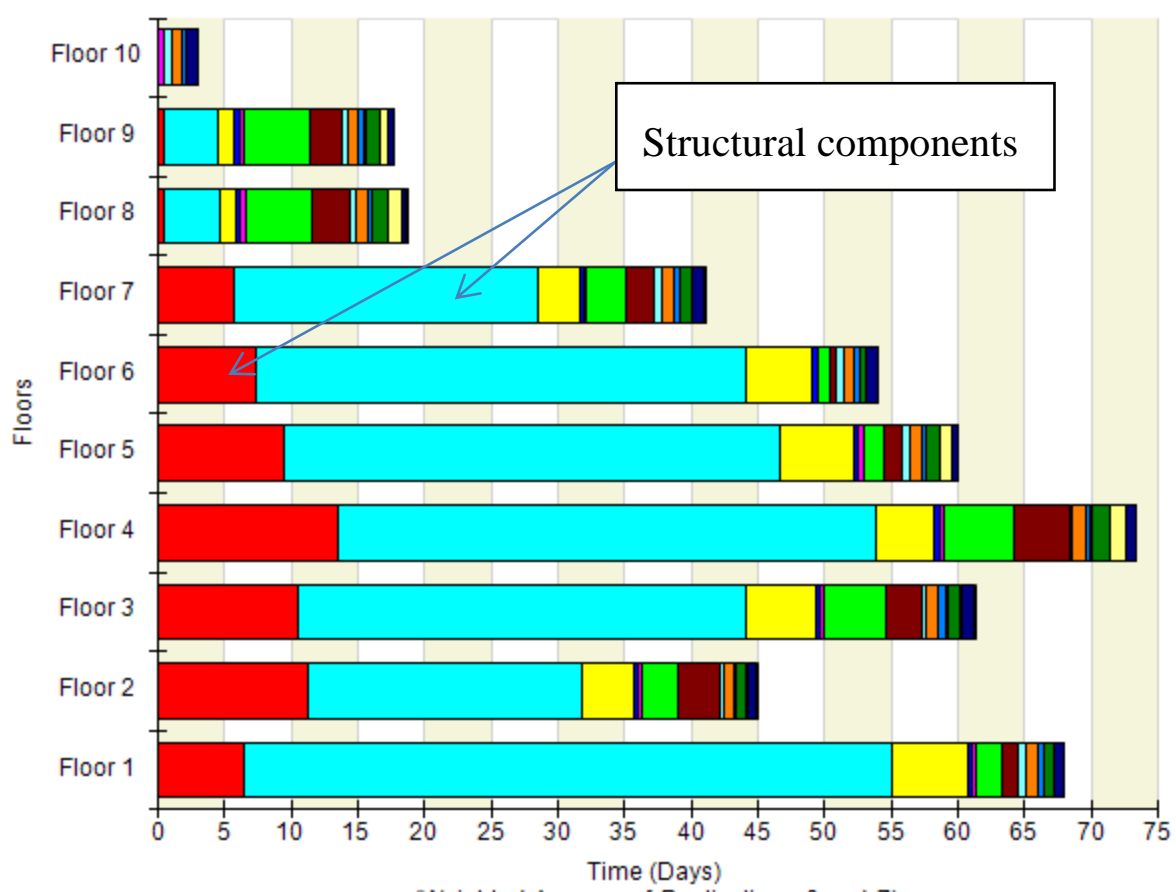

(a)

(Weighted Average of Realizations 6 and 7)

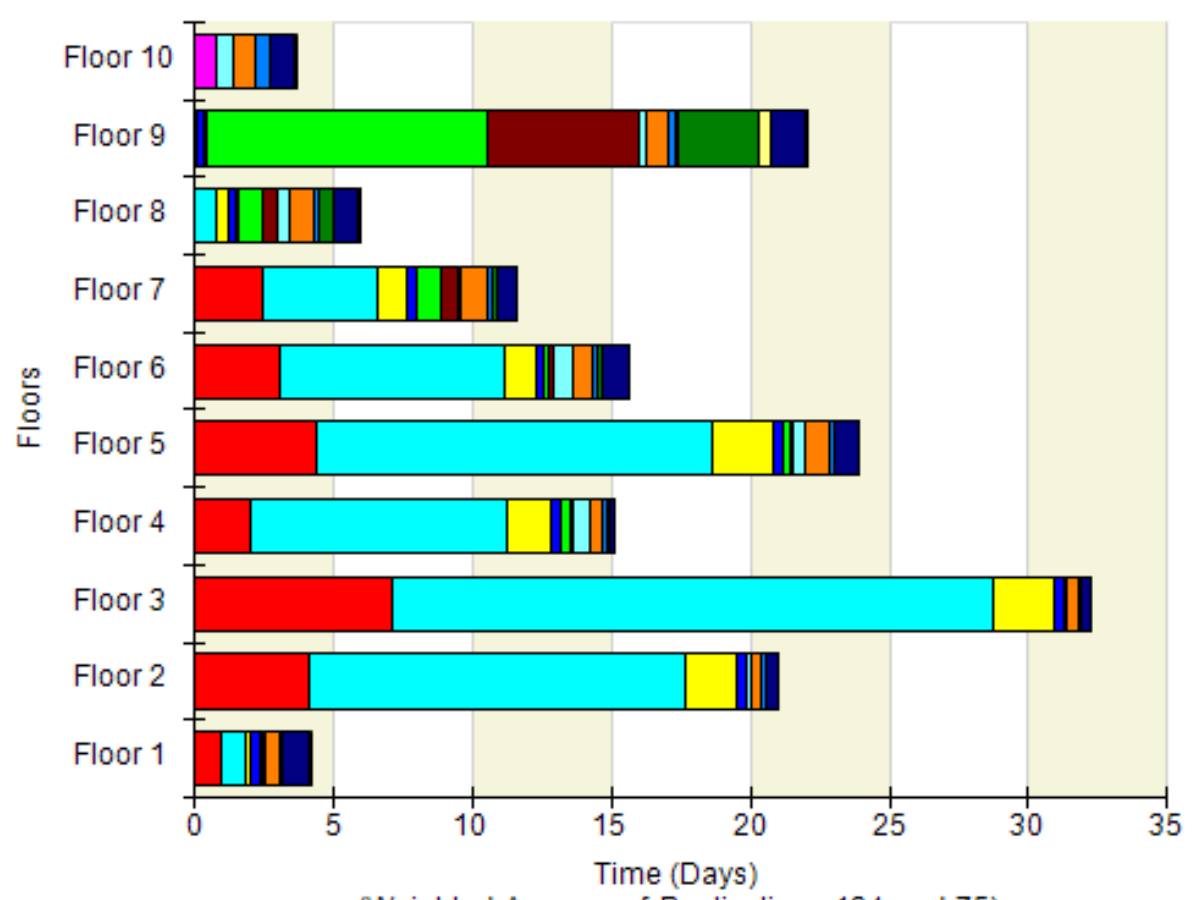

(b)

(Weighted Average of Realizations 134 and 75)

Figure 9-9 Story-wise distribution of repair times at DBE level: (a) SMRF and (b) SVD frames 


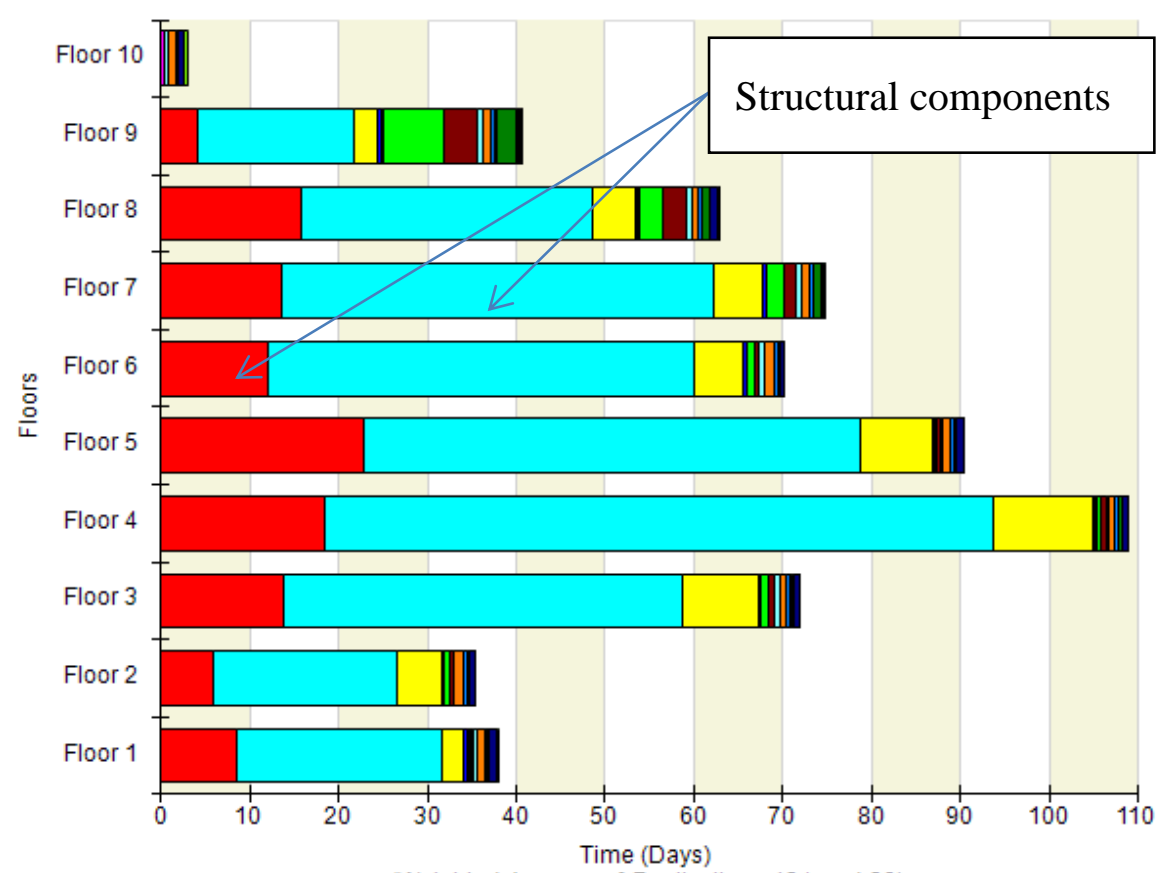

(a)

(Weighted Average of Realizations 134 and 38)

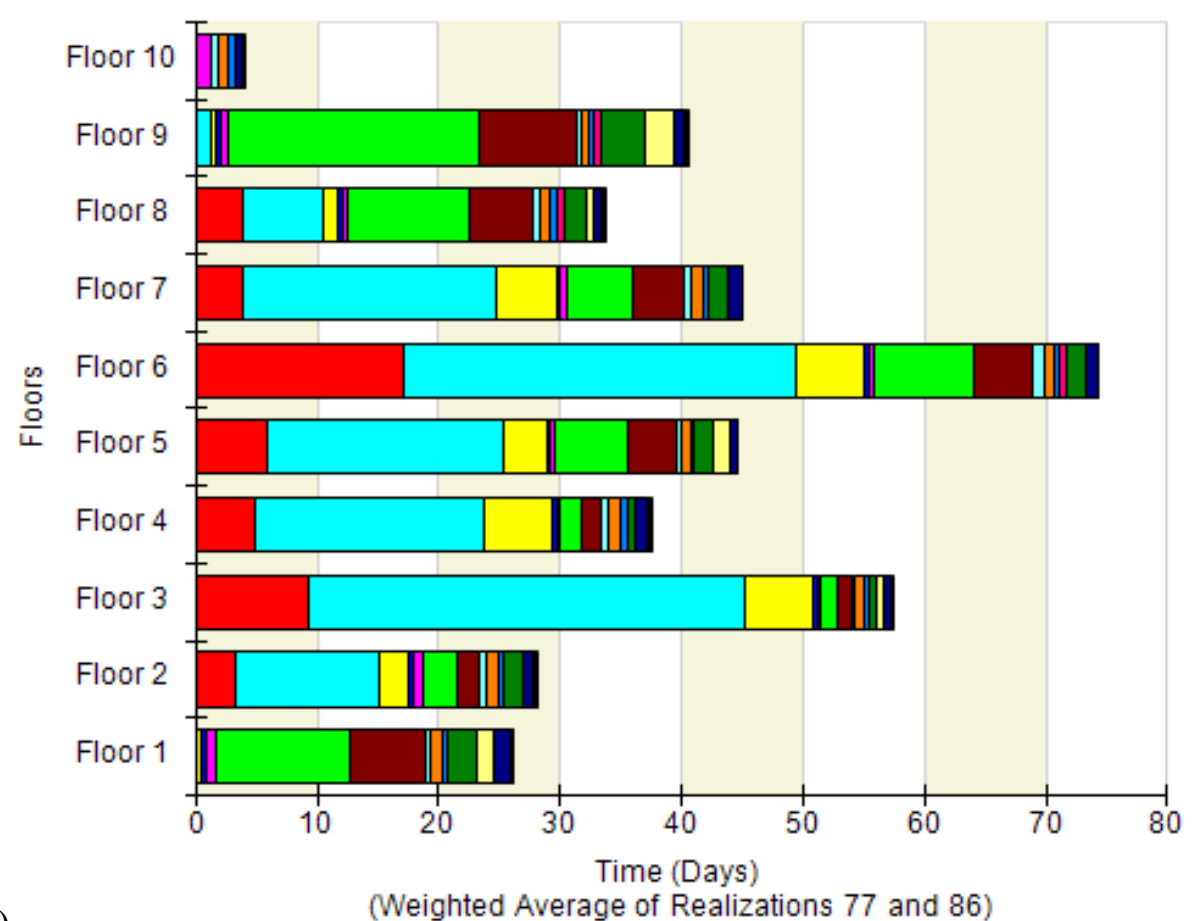

(b)

Figure 9-10 Story-wise distribution of repair times at MCE level: (a) SMRF and (b) SVD frames 


\subsubsection{Repair Cost with Realizations}

Figure 9-11 shows the repair cost required for the SMRF and SVD frames from 200 realizations at the DBE hazard level. As shown in Figure 9-11(a), the SMRF indicates higher total repair cost caused by the residual drift and the collapse. It can be observed that the SVD frame shows a lower total repair cost, compared to the repair cost for the SMRF, demonstrating the higher performance of the SVD frame. Due to the self-centering behavior and good energy dissipation capacity of the SVD frames, the SVD frame minimizes repair costs caused by no or lower residual drift and collapse.

Figure 9-12 illustrates the total repair cost for the SMRF and SVD frames from 200 realizations at the MCE level of seismic hazard. Figures Figure 9-12 (a) and (b) highlight that the SVD frame requires a lower total repair cost, compared to the SMRF, at the MCE level. Figure 9-12(a) illustrates that the SMRF demands higher total repair costs caused by its higher residual drift and collapse. The SMRF costs more to repair the structural and nonstructural components damaged at the MCE level. The SVD frame requires a lower total repair cost due to higher collapse capacity $\left(\mathrm{S}_{C T}=0.87 \mathrm{~g}\right)$ and the prevention of collapse.

Performance results from PACT software show that the total repair cost of the SMRF is considerably controlled by the residual drift, rather than collapse, at both the DBE and MCE levels of seismic hazard, as shown in Figures 9-11 and 9-12. Building components can be considered irreparable when the damage is caused by residual drift. The SMRF indicates irreparable damage caused by the excessive residual drift at the DBE and MCE levels of seismic hazard, which emphasizes the higher repair cost associated with residual drift. However, the SVD frame reveals no repair cost associated with either collapse or residual drift at the DBE and MCE levels of seismic hazard, which illustrates the superior performance of the SVD frame in minimizing the damage and collapse potential, due to lower residual drift caused by the SVD system. Hence, the

steel building frame installed with SVDs demonstrates superior performance by reducing the damage associated with the residual drift and the collapse of structural components. 


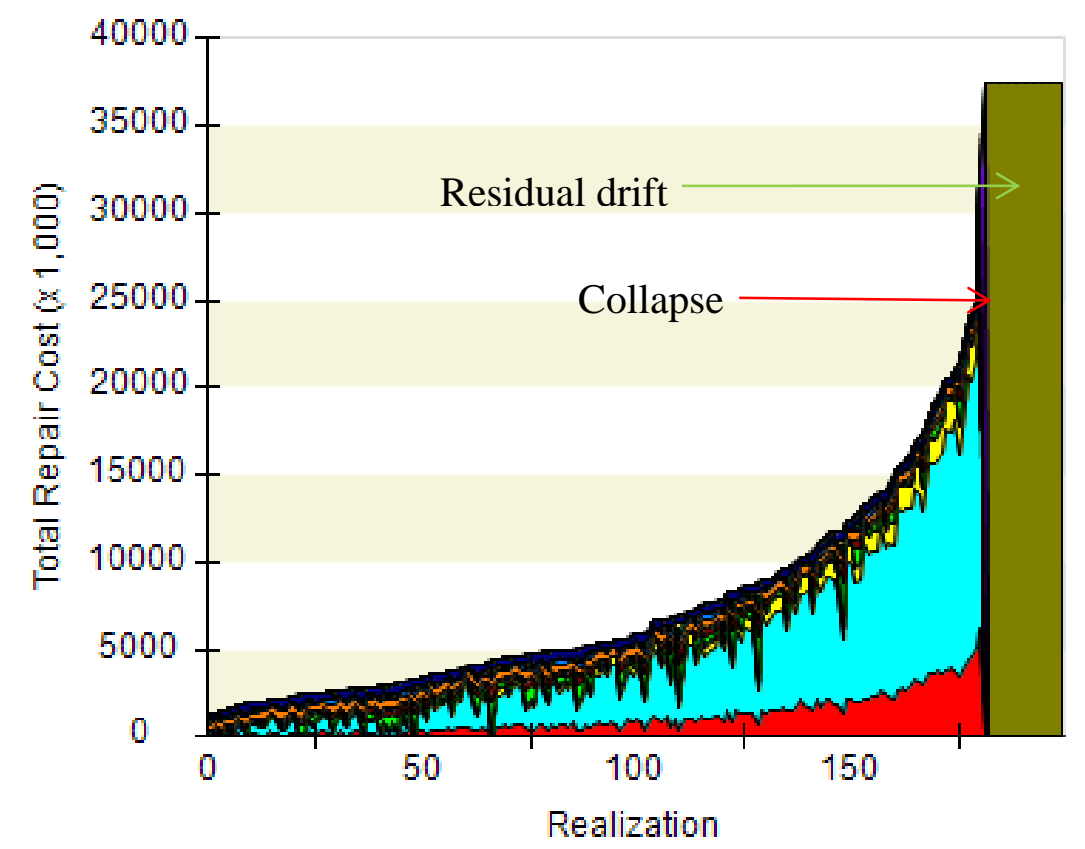

(a)

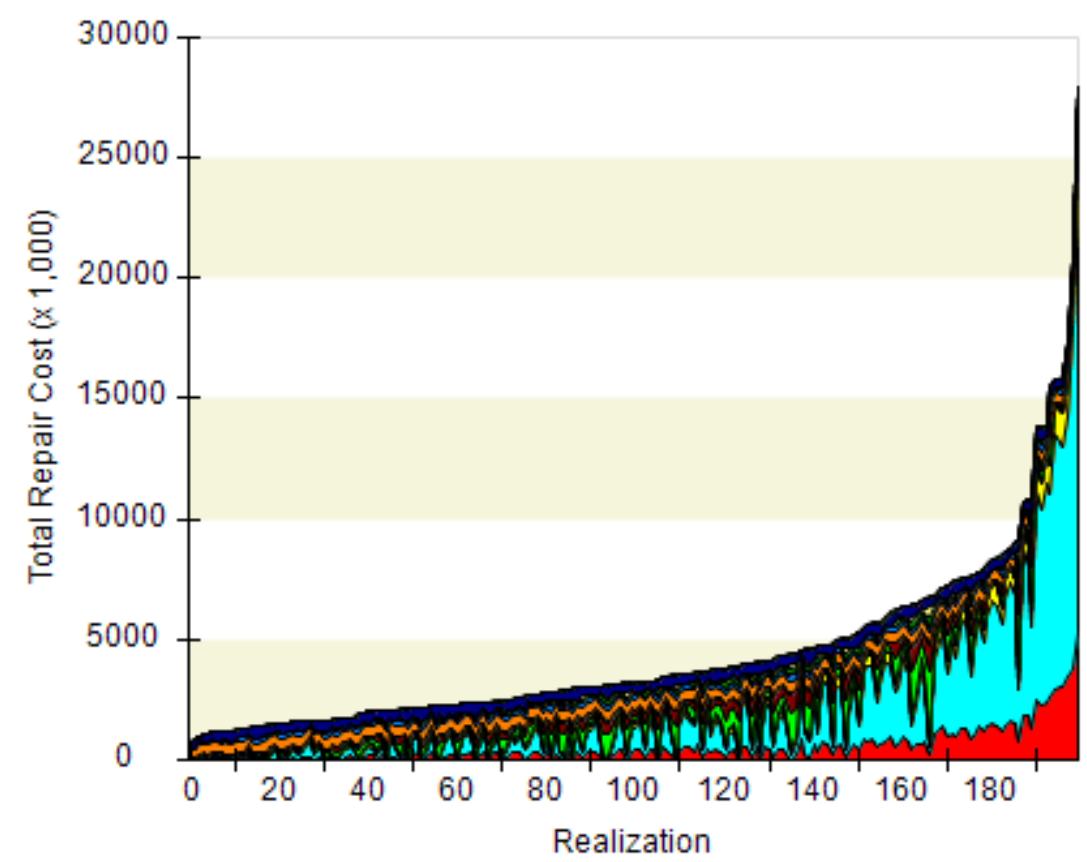

(b)

Figure 9-11 Repair costs from 200 realizations at the DBE level for (a) SMRF and (b) SVD frames 


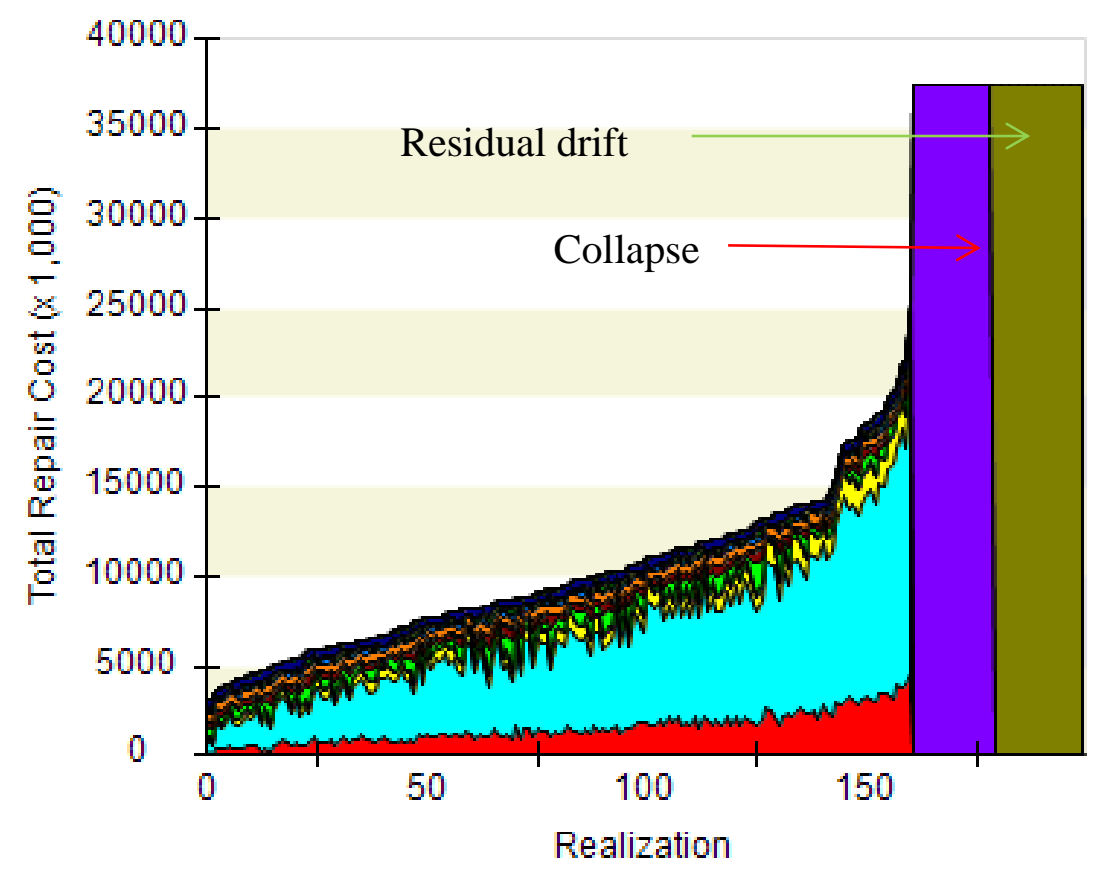

(a)

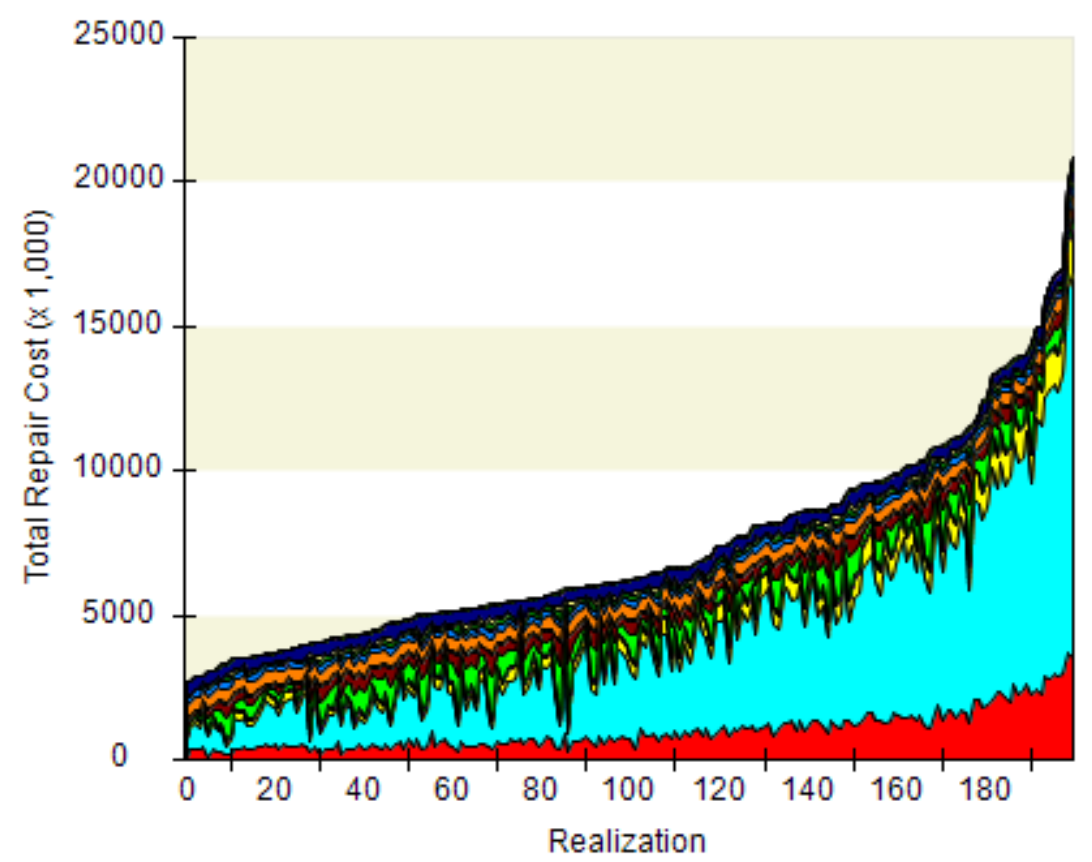

(b)

Figure 9-12 Repair costs from 200 realizations at the MCE level for (a) SMRF and (b) SVD frames 


\subsubsection{Disaggregation of Building Loss}

The PACT toolbox also provides a time-based performance assessment to obtain a building loss curve that reflects the total expected losses as a percentage of building replacement cost. Figures Figure 9-13 (a) and (b) illustrate the building loss curve disaggregation for the SMRF contributions from different intensity measures. As shown in Figure 9-13 (a), the building loss curve for the SMRF plots the total expected loss related to building repair cost as a function of probability of exceedance of repair cost, at different intensity measures. The annualized repair cost and the repair time for the SMRF are \$36,837.70 and 0.32 days as shown in Figure 9-13.

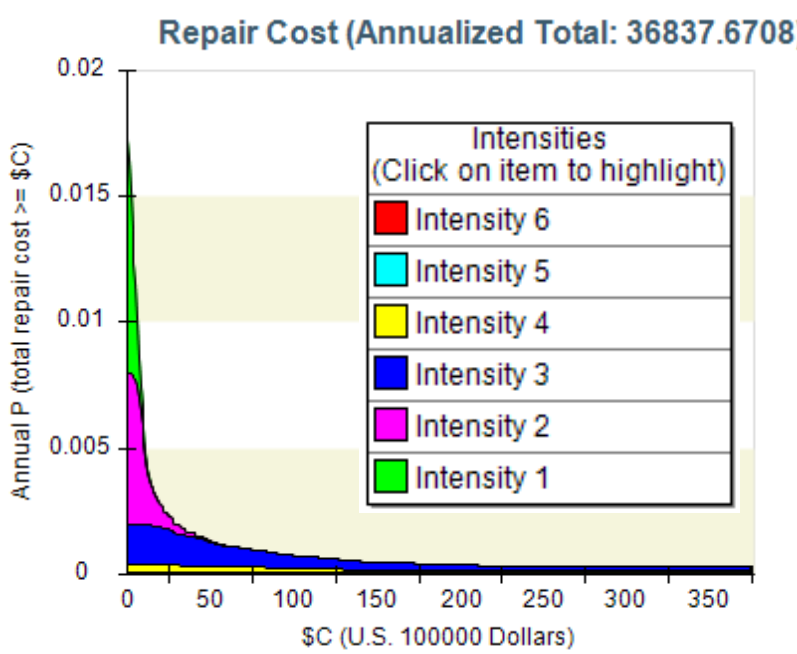

(a)

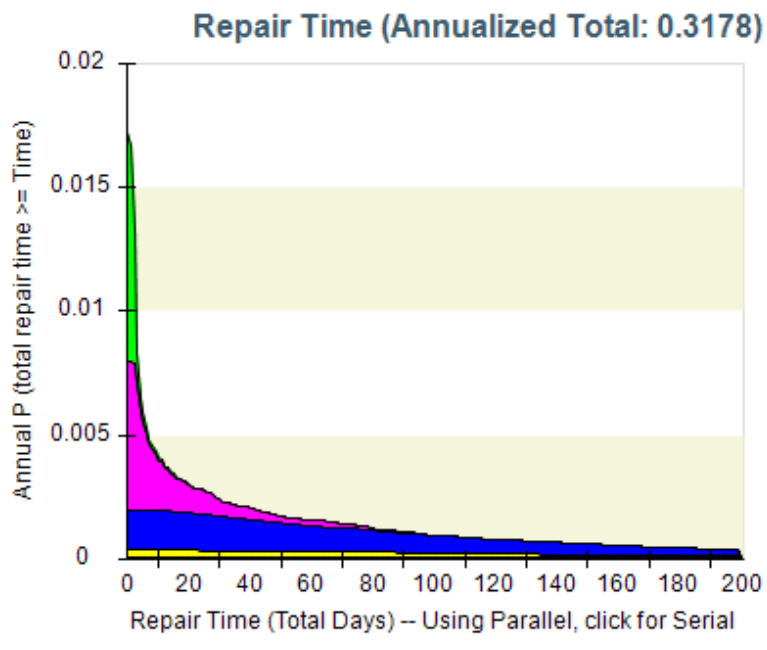

(b)

Figure 9-13 Disaggregation of the building loss curve into contributions from different intensity measures for SMRF

Figures Figure 9-14 (a) and (b) display the time-based performance assessment of the SVD frame, indicating a building loss curve disaggregated in contributions from six intensity measures. The annualized repair costs and repair time for the SVD frame are $\$ 21,404.50$ and 0.19 days as shown in Figure 9-14, expressing the total expected repair loss as a function of the annual probability of exceedance of the repair cost of varying amounts. 


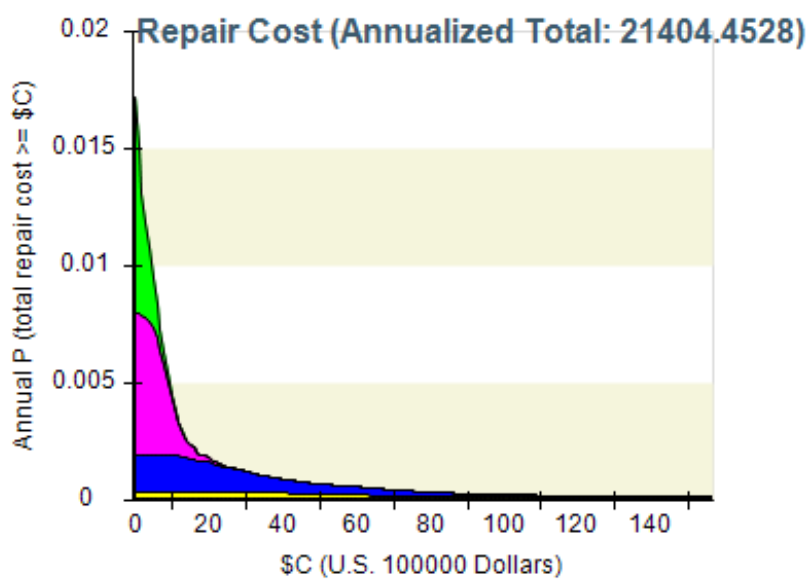

(a)

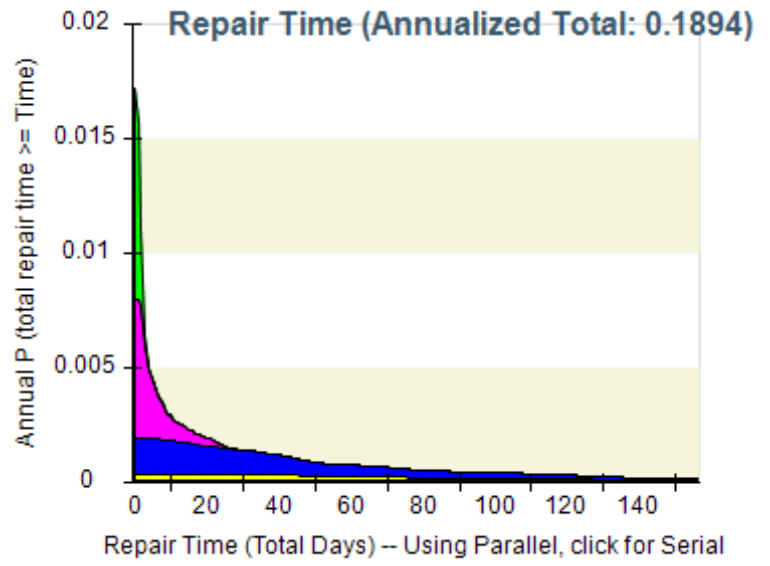

(b)

Figure 9-14 Disaggregation of the building loss curve into contributions from different intensity measures for the SVD frame

The comparison of time-based performance assessments from the PACT software shows that the SVD frame has superior performance, indicated by lower annualized repair costs and repair time. It can be observed that the annualized repair costs and repair time for the SVD frame are reduced by $41.9 \%$ and $40.4 \%$, compared to the results for the SMRF.

\subsection{Closure}

This section evaluates the potential of steel buildings upgraded with SVD in reducing economic losses under strong earthquake events. A nine-story steel building designed as a conventional SMRF or with SVDs in order to satisfy the drift requirements based on modern seismic design criteria is selected for the case study. Next, nonlinear time history analyses for both the SMRF and SVD frames are conducted under 10 far-field ground motion records to generate structural response data at different hazard levels. Then, these structural response data at six different hazard levels, and the collapse capacity of each frame system, are assigned in PACT software. The economic losses are evaluated in terms of total repair costs and total repair time at six different seismic hazard levels. 
The result shows that the steel frame system installed with SVDs reduces total repair costs and repair time at different hazard levels. It can be seen that the SVD frame reveals a lower repair cost and repair time associated with the collapse and residual drift, compared to the SMRF. The result highlights the significance of reducing residual drift as a major demand parameter to make decisions whether buildings can be repaired or need to be demolished in the aftermath of a strong earthquake. The SVD frame also contributes to lower annualized repair losses, compared to the SMRF. The application of SVDs in steel building structures can considerably reduce the repair costs of building structures at different hazard levels, indicating their enhanced post-earthquake functionality in frame buildings. 


\section{SUMMARY, CONCLUSIONS, AND RECOMMENDATIONS}

\subsection{Summary and Conclusions}

Passive energy dissipation systems can favorably affect the dynamic response of civil structures in retrofit scenarios as well as in new designs. A number of passive energy dissipation systems have been proposed and developed to mitigate the damaging effects of natural hazards. These passive energy dissipation devices can be grouped into two main categories: displacementdependent and rate-dependent devices. Examples of displacement-dependent devices include metallic yielding devices and friction devices. Energy dissipation in hysteretic devices depends primarily on the relative displacement within a device. These devices add initial stiffness until the yielding or slip occurs and dissipates energy, especially at large deformations. Metallic devices usually have a limited number of working cycles and may require replacement after a strong seismic event. Similarly, friction devices may lead to permanent deformations if no restoring force mechanism is provided in the system. Examples of rate-dependent devices encompass fluid viscous dampers and viscoelastic dampers. These rate-dependent devices can dissipate energy at all levels of vibration and may provide some stiffness. The energy dissipation capacity of ratedependent devices depends on the velocity across the device.

Viscoelastic (VE) dampers comprise viscoelastic layers, which are typically copolymers or glassy substances, bonded with steel plates. Damping is produced via the hysteresis or relative motion of polymer molecules. Some materials, such as butyl and silicone, have inherently high damping and are quite common for VE dampers. Other materials, such as natural rubber and neoprene, are compounded to produce high damping via fillers (oil, carbon black, and so on), but this may compromise other properties, such as tensile strength and elongation. A number of researchers examined the performance of VE dampers in reducing the seismic response of structures. Viscoelastic dampers have also been combined with displacement-dependent devices to produce a hybrid damping system, which can effectively control the response of structures over a wide range of displacement amplitudes. The VE dampers can provide energy dissipation at lowlevel vibrations, while the displacement-dependent devices do not provide sufficient damping at small vibrations. On the other hand, displacement-dependent devices dissipate significant energy 
during large-magnitude earthquakes and can augment the damping capacity of VE dampers during strong earthquakes. However, in all previously proposed hybrid control systems, the displacementdependent device needs to be replaced after a strong earthquake event.

This dissertation presents investigations of a shape memory alloy-based self-centering system, termed a superelastic viscous damper, to mitigate the structural responses of steel building structures subjected to multi-level seismic hazards. The superelastic viscous dampers leverage the inherent re-centering capability of shape memory alloy cables and the energy dissipation ability of a heavily damped butyl compound, which provides high damping at low stiffness, for superior seismic performance. The SMA cables recently developed as a new structural element exploit the excellent mechanical properties of thin wires to resist large axial loads. They possess advantageous characteristics, such as large tensile strength, ability to fully recover deformations up to $7 \%$ strain, and ease of handling. In addition, they present considerable cost advantages over same-size monolithic SMA bars, as they leverage the highly-optimized manufacturing process currently available for wires. Therefore, SMA cables are adopted in the proposed SVD. The viscoelastic component of the SVD is expected to provide energy dissipation without adding significant stiffness. The butyl rubber is a synthetic rubber produced by polymerization of about $98 \%$ isobutylene with about $2 \%$ of isoprene. The high damped (HD) butyl compounds are highly loaded, meaning that the percentage of rubber hydrocarbon is lower than in traditional industrial butyls. They are compounded specifically to produce high damping at moderate to low stiffness. Thus, the HD butyl is selected as the elastomer compound to be used for the SVD.

First, the experimental component testing of the SVDs is discussed and the conceptual design of the SVD is described. Then, a set of numerical studies is conducted to evaluate the efficiency of the SVDs in controlling the response of steel frame buildings. All numerical models are developed using the nonlinear seismic analysis program OpenSees. All building models capture the degradation of structural strength and stiffness of the steel frame elements associated with structural damage. The SVD is modeled by combining an element with the uniaxial selfcentering material properties to represent the SMA cables, and an element with Maxwell material properties to represent the HD butyl compound. The model parameters for the SMA cables and the elastomeric compound are selected based on the experimental test results on the NiTi SMAs and HD butyl compound. Then, the following numerical investigations are conducted: 
Retrofitting steel buildings with SVDs: A six-story special steel moment frame structure, which exhibits excessive story drifts, is retrofitted using SVDs. Nonlinear response history analyses are conducted, and the peak response quantities of both the initial frame and retrofitted frame are evaluated under a suite of 44 far-field strong ground motion records.

Designing steel buildings with SVDs: A nine-story steel building is selected from the SAC steel project for extensive numerical analyses. First, the nine-story steel frame is designed as a conventional special moment resisting frame (SMRF) to meet current seismic design requirements. Next, the reduced-strength version of the fully code-compliant frame is also developed, which satisfies the strength requirements of the design codes but does not meet the drift limits. In order to meet the story drift requirements according to ASCE 7-10, the reduced-strength frame is designed with SVDs to achieve a performance similar to that of the conventional SMRF. To evaluate the seismic performance of steel frame buildings with SVDs, a number of studies, summarized below, are conducted:

- Seismic collapse assessment: Incremental dynamic analysis is employed to access the collapse resistance of a nine-story steel frame structure designed as SMRF and SVD frames. For comparison purposes, the steel building is also designed with buckling restrained braces (BRB). The performance of each system in mitigating the response of steel frame buildings under various levels of seismic hazard is assessed. A sensitivity analysis is also conducted to evaluate the effect of SVD design parameters on the collapse, and overall performance of steel frames designed with SVDs.

- Fragility curve estimates: The structural responses of steel frame buildings under various seismic hazard levels are evaluated by conducting nonlinear response history analyses. Probabilistic demand models are then developed for inter-story drift, residual inter-story drift and peak floor acceleration. In particular, in order to incorporate different types of data (i.e., equality data and lower bound data) obtained from the numerical analysis, the maximum likelihood approach is applied to assess the model parameters. With the developed demand models, seismic fragility curves at different performance levels are created for both SMRF and SVD frames to assess the effectiveness of the SVDs, and the structural fragilities are 
proposed to be evaluated based on a union of two limit states defined by inter-story drift and residual inter-story drift, respectively.

- Aftershock fragility assessment: Incremental dynamic analysis is conducted for both frames to identify the structural response under mainshock events scaled to increasing intensities. A suite of historical mainshock and aftershock ground motion records is employed in the analysis. Three post-mainshock damage states are defined based on maximum inter-story drift thresholds, and the corresponding aftershock collapse fragility analyses are performed at each damage state.

- Effect of the temperature on SVDs: The parameters of SVD models are adjusted for three temperatures: $0^{\circ} \mathrm{C}, 23^{\circ} \mathrm{C}$ and $40^{\circ} \mathrm{C}$, and nonlinear time history analyses of the steel frame building installed with SVDs are conducted at each temperature point to determine peak response quantities.

- Seismic loss assessment: Finally, the seismic loss assessment of steel frame buildings with SVDs is investigated, employing the PACT software provided in the performance-based seismic assessment methodology presented in the FEMA P-58. Nonlinear time history analysis results for both SMRF and SVD frames are generated at different hazard levels under ten far-field ground motion records. These structural response data and the collapse capacity of each frame are provided in the PACT software. Then, the seismic economic losses are compared for both frames in terms of total repair cost and repair time, at six different hazard levels, to evaluate the effectiveness of the SVD device.

The results from these investigations revealed that steel frame buildings designed or retrofitted with SVD systems can considerably improve structural performance, especially at high seismic hazard levels, while providing almost the same performance for the acceleration-sensitive non-structural components with traditional SMRF systems. Residual drifts are significantly reduced for the frames with SVDs. The seismic loss analysis result indicates that the frame installed with SVDs has a superior seismic performance at different hazard levels, indicated by lower total repair cost and repair time. Reducing residual drifts improves the seismic performance of frame buildings by lowering the repair cost or avoiding the demolition of damaged structures after the occurrence of strong earthquake events. In addition, aftershock analyses indicate the advantages of the self-centering systems in reducing seismic losses and risks in steel structures 
when they are subjected to seismic sequences. Hence, the application of SVDs in building structures can significantly reduce post-earthquake losses. It is shown that the change in environmental temperature does not significantly affect the peak drift and acceleration response of these buildings.

\subsection{Recommendations for Future Studies}

The present study investigates the seismic performance of a superelastic viscous damper through extensive numerical simulations in steel building structures. Future studies of the proposed device need to conduct experimental investigations to reinforce further understanding of the behavior and performance by testing a prototype device in the laboratory. Furthermore, additional analytical studies can be conducted to evaluate the performance of the proposed hybrid device installed in steel building structures having different story configurations.

The numerical studies reported here evaluates the performance of steel building structures designed with SVDs considering the degradation effects only on the beam and columns of frame elements but not in the SVD device. Future studies can also evaluate the performance of building structures designed with SVDs considering the effect of degradation in SVD response under cyclic loading in addition to the inclusion of strength and stiffness degradation of beam and column elements. Considering degradation effects on shape memory alloy and viscous damper elements can augment to understand the collapse behavior of building structures designed using SVDs. This study assumes the complete collapse of beam and column elements of the building before the collapse of the SVD device. Finally, note that at extreme large responses, it is assumed that not only the SVDs but also complete building is considered to fail.

The SVD device is developed as a new hybrid control device that works on the principle of both displacement-dependent and rate-dependent control devices. There is a need to develop a simplified design procedure for the proposed hybrid SVD device. In the literature, the simplified design procedure for rate-based devices such as the viscoelastic/elastomeric dampers (Lee et al., 2005) or displacement-based devices such as friction damper (Min et al., 2010) are available. Hence, the simplified design procedure and/or direct displacement-based design method can be developed to design the proposed hybrid device. 
The proposed investigation is concentrated on the seismic performance evaluation of steel frame structures. In particular, future study can also be extended to evaluate the performance of reinforced concrete frame structures with installed SVD devices, which can provide further insights to comprehend the seismic behavior of the SVD devices installed in different types of frame structures. In addition, the future research work can also be conducted for irregular and complex frame building structures with different story heights and configurations to investigate the performance of the SVD devices installed under different structural configurations. Such investigations can indicate the distribution of a later-load resistance capacity and hinge formation mechanism of building structures installed with SVD devices.

This dissertation work is focused on the seismic performance evaluation of frame building structures subjected to far-field ground motion records and mainshock-aftershock sequences consisting of only far-field records. Furthermore, future research work can be extended to evaluate the performance of SVD devices installed in buildings subjected to near field-ground motions that are characterized by distinct pulse-like time histories, ground motion records with directivity effect, and high ratio of peak ground velocity and peak ground acceleration. Furthermore, the performance evaluation can be carried out for the mainshock-aftershock sequences that include the near-field and far-field ground motion records.

The present study evaluates the collapse performance of steel frame buildings considering inter-story drift as a main engineering demand parameter. Since the SVD device can reduce the residual drifts significantly compared to the conventional moment resisting frames, the future research for collapse evaluation can be conducted considering residual drift as an another important engineering parameter to further understand the insights of structural performance of frame structures installed with SVDs.

Finally, please note that this study follows the current numerical analysis practice for the seismic evaluation, which is to subject structures to in-plane seismic excitations. As shown in other studies (e.g, Xu and Gardoni, 2016), a 3D analysis can provide more accurate fragility estimates. Therefore, the $3 \mathrm{D}$ analyses under bi-axial ground motion records need to be considered in future studies. 


\section{REFERENCES}

AISC Committee (2010). "Specification for Structural Steel Buildings (ANSI/AISC 36010.” American Institute of Steel Construction, Chicago-Illinois.

AISC. (2005). "Seismic provisions for structural steel buildings. ANSI/AISC 341-05." American Institute for Steel Construction, Chicago, Illinois; 2005.

Alam, M. S., Youssef, M. A., and Nehdi, M. (2008). "Analytical prediction of the seismic behaviour of superelastic shape memory alloy reinforced concrete elements." Engineering Structures, 30(12), 3399-411.

Alaneme, K. K., and Okotete, E. A. (2016). "Reconciling viability and cost-effective shape memory alloy options-A review of copper and iron based shape memory metallic systems.” Engineering Science and Technology, an International Journal, 19(3), 1582-92.

Araki, Y., Maekawa, N, Omori, T., Sutou, Y., Kainuma, R., and Ishida, K. (2012). "Ratedependent response of superelastic $\mathrm{Cu}-\mathrm{Al}-\mathrm{Mn}$ alloy rods to tensile cyclic loads." Smart Mater. Struct, 21(3), 032002.

Araki, Y., Shrestha, K. C., Maekawa, N., Koetaka, Y., Omori, T. and Kainuma, R., (2016). "Shaking table tests of steel frame with superelastic $\mathrm{Cu}-\mathrm{Al}-\mathrm{Mn}$ SMA tension braces." Earthquake Engineering \& Structural Dynamics, 45(2),.297-314.

ASCE. (2005). "Minimum design loads for buildings and other structures." ASCE 7-05. American Society of Civil Engineers, Reston, Virginia.

ASCE. (2007). "Seismic Rehabilitation of Existing Buildings (41-06)." American Society of Civil Engineers, Reston VA.

ASCE. (2010): "Minimum Design Loads for Buildings and Other Structures." ASCE 7-10. American Society of Civil Engineers, Reston, Virginia.

Asgarian, B., Moradi, S. (2011). "Seismic response of steel braced frames with shape memory alloy braces.” Journal of Constructional Steel Research, 67(1), 65-74.

Azadi, B., Rajapakse, R. K. N. D., and Maijer, D. M. (2006). "One-dimensional thermomechanical model for dynamic pseudoelastic response of shape memory alloys." Smart Materials and Structures, 15, 996-1008.

Badpay, A., and Arbabi, F. A. (2008). "Comparison of seismic performance and vulnerability of buckling restrained and conventional steel braced frames." In: Proceedings of the 14th world conference on earthquake engineering, Beijing, China, October 2008.

Basoz, N., and Kiremidjian, A. S. (1999). "Development of empirical fragility curves for bridges." Technical Council on Lifeline Earthquake Engineering Monograph Proceedings of the 1999 5th U.S. Conference on Lifeline Earthquake Engineering: Optimizing PostEarthquake Lifeline System Reliability 1999, August 12-14, 693-702.

Bhowmick S., and Mishra S. K. (2016). "FNCATB Superelastic damper for seismic vibration mitigation.” Journal of Intelligent Material Systems and Structures, 27(15), 2062-2077. 
Bhowmick, S., Mishra, S. K. (2016). "FNCATB Superelastic damper for seismic vibration mitigation.” Journal of Intelligent Material Systems and Structures, 27(15), 2062-77.

Bhuiyan, A. R., Alam, M. S. (2013). "Seismic performance assessment of highway bridges equipped with superelastic shape memory alloy-based laminated rubber isolation bearing." Engineering structures, 49, 396-407.

Billah, A. H. M. M. D. (2015). "Performance-based seismic design and assessment of concrete bridge piers reinforced with shape memory alloy rebar" Doctoral dissertation, University of British Columbia).

Billah, A. M., Alam, M. S. (2013). "Seismic performance of concrete columns reinforced with hybrid shape memory alloy (SMA) and fiber reinforced polymer (FRP) bars." Construction and Building Materials, 28(1), 730-42.

Bojórquez, E., and Ruiz-García, J. (2013). "Residual drift demands in moment-resisting steel frames subjected to narrow-band earthquake ground motions." Earthq. Eng. Struct. Dyn., 42(11) 1583-98.

Buehler, W. J., Gilfrich, J. V., and Wiley, R. C. (1963). "Effect of low-temperature phase changes on the mechanical properties of alloys near composition TiNi." Appl Phys, 34, 1475-7.

Cai, W., Lu, X. L., and Zhao, L. C. (2005). "Damping behavior of TiNi-based shape memory alloys." Materials Science and Engineering, A, 394(1) 78-82.

Carboni, B., Lacarbonara, W., and Auricchio, F. (2015). "Hysteresis of multi configuration assemblies of nitinol and steel strands: Experiments and phenomenological identification" J. Eng. Mech., 10.1061/(ASCE) EM.1943-7889.0000852, 04014135.

Chang, B. C., Shaw, J. A., and Iadicola, M. A. (2006). "Thermodynamics of shape memory alloy wire: modeling, experiments, and application." Continuum Mechanics and Thermodynamics, 18(1), 83-118.

Chen, W., and Bo, S. (2006). "Temperature dependence of a NiTi shape memory alloy's superelastic behavior at a high strain rate." Journal of Mechanics of Materials and Structures, 1(2), pp.339-356.

Chen, Y., Jiang, H. C., Liu, S. W., Rong, L. J., and Zhao X. Q. (2009). "Damping capacity of TiNibased shape memory alloys." Journal of Alloys and Compounds, 482(1), pp.151-154.

Choi, E., DesRoches, R., Nielson, B. G. (2004). "Seismic fragility of typical bridges in moderate seismic zones.” Eng. Struct., 26 (187-199) (2004).

Churchill, C.B., Shaw, J.A. and Iadicola, M.A. (2009). "Tips and tricks for characterizing shape memory alloy wire: part 2-fundamental isothermal responses." Experimental Techniques, 33(1), pp.51-62.

Clarke, J, Tesfamariam, S, Yannacopoulos, S. (2009). "Smart structures using shape memory alloys." InSPIE Smart Structures and Materials + Nondestructive Evaluation and Health Monitoring (pp. 729205-729205). International Society for Optics and Photonics.

Decanini, L.D., Liberatore, D., Liberatore, L. and Sorrentino, L. (2015). "Preliminary report on the 2012, May 20, Emilia earthquake." Vol. 1, 2012. 
DesRoches, R., and Smith, B. (2004). "Shape Memory Alloys in Seismic Resistant Design and Retrofit: A Critical Review of Their Potential and Limitations." Journal of Earthquake Engineering, 8(3), 415-429.

DesRoches, R., Comerio, M., Eberhard, M., Moony, W., and Rix, G. J. (2011). "Overview of the 2010 Haiti earthquake Earthq." Spectra, 27(S1) S1-S21.

DesRoches, R., McCormick, J., Delemont, M. (2004). “Cyclic properties of superelastic shape memory alloy wires and bars." Journal of Structural Engineering, 2004 Jan;130(1):38-46.

Dezfuli, F. H., and Alam, M. S. (2015). "Hysteresis model of shape memory alloy wire-based laminated rubber bearing under compression and unidirectional shear loadings." Smart Mater. Struct., 24(6) 065022

Dezfuli, F.H., and Alam, M.S. (2013). "Shape memory alloy wire-based smart natural rubber bearing." Smart Materials and Structures, 22(4), p.045013.

Dolce, M., and Cardone, D. (2001). "Mechanical behaviour of shape memory alloys for seismic applications 2. Austenite NiTi wires subjected to tension." International Journal of Mechanical Sciences, 43(11), pp.2657-2677.

Dutta, A., Mander, J. B. (1998). "Seismic fragility analysis of highway bridges. Center-to-Center Project Workshop on Earthquake Engineering Frontiers in Transportation Systems." Tokyo, Japan, International Center for Disaster-Mitigation Engineering (INCEDE), 1998.

Dyanati, M., Huang, Q., and Roke, D. (2016). "Cost-Benefit Evaluation of Self-centering Concentrically Braced Frames Considering Uncertainties." Structure and Infrastructure Engineering, DOI: 10.1080/15732479.2016.1173070.

Dyanati, M., Huang, Q., Roke, D. (2015). "Seismic demand models and performance evaluation of self-centering and conventional concentrically braced frames." Eng. Struct., 84 (368381) (2015).

Eatherton, M. R., and Hajjar, J. F. (2011). "Residual drifts of self-centering systems including effects of ambient building resistance." Earthquake Spectra, 27(3), 719-744.

EERI. (2011". "Tohoku Japan Earthquake \& Tsunami Clearing House M9.0 March 2011." http://www.eqclearinghouse.org/2011-03-11-sendai/category/field-blog/

Ellingwood, B. R., Wen Y.K. (2005). "Risk-benefit-based design decisions for lowprobability/high consequence earthquake events in Mid-America." Prog. Struct. Eng. Mat., 7 (56-70) (2005).

Erochko J, Christopoulos C, Tremblay R and Choi H 2010 Residual drift response of SMRFs and BRB frames in steel buildings designed according to ASCE 7-05 J. Struct. Eng. 2010 137(5) 589-99

FEMA, P-751. 2009. "NEHRP recommended seismic provisions: design examples." Federal Emergency Management Agency, Washington, DC; 2012.

FEMA. (2000). "Recommended Seismic Design Criteria for New Steel Moment Frame Buildings (FEMA 350).” Federal Emergency Management Agency, Washington DC. 
FEMA. (2000). "State of the Art Report on Systems Performance of Steel Moment Frames Subject to Earthquake Ground Shaking (FEMA 355C)." Federal Emergency Management Agency, Washington DC.

FEMA. (2009). "Quantification of building seismic performance factors. Report No. P695." Federal Emergency Management Agency, Washington, D.C.

FEMA. (2012). "Seismic performance assessment of buildings Report FEMA P-58 Vol. 1Methodology." Federal Emergency Management Agency, Washington DC.

FEMA. (2014). "HAZUS-MH 2.1 Technical Manual: Earthquake Model." Federal Emergency Management Agency, Washington DC.

Gardoni, P., Mosalam, K. M., Kiureghian, A. Der. (2003). "Probabilistic seismic demand models and fragility estimates for RC bridges." J. Earthq. Eng.. 7 (79-106) (2003).

Ghodke, S., Jangid, R.S. (2016). "Influence of high austenite stiffness of shape memory alloy on the response of base-isolated benchmark building." Structural Control and Health Monitoring.

Goda, K., Pomonis, A., Chian, S. C., Offord, M., Saito, K., Sammonds, P., Fraser, S., Raby, A., and Macabuag, J. (2011). "Ground motion characteristics and shaking damage of the 11th March 2011 M w9. 0 Great East Japan earthquake.” Bull. Earthq. Eng. 11(1) 141-70.

Gong, J. M., Tobushi, H., Takata, K., Okumura, K., and Endo, M. (2002). "Cyclic superelastic deformation of TiNi shape-memory alloy.” In Materials Science Forum, 394, 245-248). Trans Tech Publications.

Graesser, E. J., Cozzarelli, F. A. (1991). "Shape-memory alloys as new materials for aseismic isolation." Journal of Engineering Mechanics, 117(11),2590-608.

Gupta, A., Krawinkler, H. (1999). "Seismic Demands for Performance Evaluation of Steel Moment Resisting Frame Structures, Technical Report 132." The John A. Blume Earthquake Engineering Research Center, Department of Civil Engineering, Stanford University, Stanford, CA.

Gur, S., Mishr, S. K., Chakraborty, S. (2014). "Performance assessment of buildings isolated by shape-memory-alloy rubber bearing: Comparison with elastomeric bearing under nearfault earthquakes." Structural Control and Health Monitoring, 21(4):449-65.

Hauksson, E., Jones, L. M. (1995). "The 1994 Northridge earthquake sequence in California: seismological and tectonic aspects." J. Geophys. Res 100(B7) 12335-12355.

He, X. M., and Rong, L. J., (2004). "DSC analysis of reverse martensitic transformation in deformed Ti-Ni-Nb shape memory alloy." Scripta Materialia, 51(1), 7-11.

Hsiao, P. C., Lehman, D. E. and Roeder, C. W. (2013). "Evaluation of the response modification coefficient and collapse potential of special concentrically braced frames." Earthquake Engineering \& Structural Dynamics, 42(10), 1547-1564.

Huang, Q., Gardoni, P., Hurlebaus, S. (2010). "Probabilistic seismic demand models and fragility estimates for reinforced concrete highway bridges with one single-column bent." ASCE J. Eng. Mech., 136 (1340-1353) (2010). 
Hwang, H., Jernigan, J. B., Lin, Y. W. (2000). "Evaluation of seismic damage to Memphis bridges and highway systems.” J. Bridge Eng., 5 (322-330) (2000).

Ibarra, L. F., Medina, R. A., Krawinkler, H. (2005). "Hysteretic models that incorporate strength and stiffness deterioration." Int J Earthq Eng Struct Dyn, 34 (12), 1489-511.

Janke, L., Czaderski, C., Motavalli, M., and Ruth, J. (2005). “Applications of shape memory alloys in civil engineering structures_overview, limits and new ideas." Materials and Structures, 38(5):578-92.

Jeon, J. S., DesRoches, R., Lowes, L. N., Brilakis, I. (2015). "Framework of aftershock fragility assessment-case studies: older California reinforced concrete building frames." Earthq. Eng. Struct. Dyn., 44(15) 2617-36.

Kafaeikivi, M., Roke, D. and Huang, Q., (2013). "Seismic performance assessment of dual systems combining conventional and self-centering concentrically braced-frames." In Proceedings of the 11th International Conference on Structural Safety \& Reliability (ICOSSAR).

Kam, W. Y., Pampanin, S., and Elwood, K. (2011). "Seismic performance of reinforced concrete buildings in the 22 February Christchurch (Lyttelton) earthquake." Bull. New Zeal. Soci. Earthq. Eng., 44(4) 239-278.

Kauffman, G., Mayo, I. (1997). "The story of Nitinol: the serendipitous discovery of the memory metal and its applications." Chem Educator, 2, 1-21.

Lee, K. S., Fan, C. P., Sause, R., Ricles, J. (2005). "Simplified design procedure for frame buildings with viscoelastic or elastomeric structural dampers." Earthquake engineering \& structural dynamics, 34(10), 1271-1284.

Leon, R. T., DesRoches, R., Ocel, J., and Hess G. (2001). "Innovative beam column connections using shape memory alloys." InSPIE's 8th Annual International Symposium on Smart Structures and Materials, 227-237. International Society for Optics and Photonics.

Li, Y., Song, R., and Van De Lindt, J. W. (2014). "Collapse fragility of steel structures subjected to earthquake mainshock-aftershock sequences.” J. Struct. Eng. 140(12), 04014095.

Lignos, D. G., and Krawinkler, H. (2010). "Deterioration modeling of steel components in support of collapse prediction of steel moment frames under earthquake loading." J. Struct. Eng., 137(11), 1291-302.

Lignos, D. G., Krawinkler, H. (2007). "A Database in Support of Modeling of Component Deterioration for Collapse Prediction of Steel Frame Structures." Proc. ASCE Structures Congress, Long Beach, California, May 18-20; 2007.

Lignos, D. G., Krawinkler, H. (2011). "Deterioration modeling of steel components in support of collapse prediction of steel moment frames under earthquake loading." J. Struct. Eng., 137 (1291-1302). DOI: 10.1061/(ASCE)ST.1943-541X.0000376

Lin, K.C., Lin, C.C.J., Chen, J.Y., Chang, H.Y. (2010). "Seismic reliability of steel framed buildings." Struct. Safet. 32 (174-182) (2010).

Liu, N., and Huang, W. M. (2006). "DSC study on temperature memory effect of NiTi shape memory alloy." Transactions of Nonferrous Metals Society of China, 16, s37-s41. 
Liu, S., and Warn, G.P. (2012). "Seismic performance and sensitivity of floor isolation systems in steel plate shear wall structures.” Eng. Struct. 42 (115-126) (2012).

Lowes, L. N., Altoontash, A. (2003). "Modeling of reinforced-concrete beam-column joints subjected to cyclic loading." J Struct Eng 129(12), ASCE.

Luo, X., Ge, H., Usami, T. (2012). "Temperature effects of shape memory alloys (SMAs) in damage control design of steel portal frames." Front Struct Civ Eng, 6 (4), 348-57.

Ma, H, Wilkinson T, Cho C. Feasibility study on a self-centering beam-to-column connection by using the superelastic behavior of SMAs. Smart Materials and Structures. 2007 Jul 27; 16(5):1555.

Ma, H., and Cho, C. (2008). "Feasibility study on a superelastic SMA damper with re-centring capability." Materials Science and Engineering: A., 473(1), 290-6.

Mackie, K., Stojadinović, B. (2003). "Seismic demands for performance-based design of bridges, PEER Rep. No. 2003/16.” Pacific Engineering Earthquake Research Center University of California, Berkeley, California.

MalÃ Šcot, P., Lexcellent, C., FoltÃ̄̄te, E., and Collet, M. (2006). "Shape memory alloys cyclic behavior: experimental study and modeling." Journal of Engineering Materials and Technology, 128(3), 335-345.

Mander, J. B., Basoz, N. (1999). "Seismic fragility curve theory for highway bridges." Fifth U.S. Conference on Lifeline Earthquake Engineering, Seattle, WA, ASCE.

MANSIDE. (1998). "Memory alloys for new structural vibrations isolating devices," MANSIDE Third Twelve Monthly Progress Report.

Mayes, R. L., Goings, C. B., Naguib, W. I., and Harris, S. K. (2005). "Comparative seismic performance of four structural systems." In: Proceedings of 74th Annual Convention of structural engineers association of California, San Diego, California, USA.

McCormick, J., Aburano, H., Ikenaga, M., and Nakashima, M. (2008). "Permissible residual deformation levels for building structures considering both safety and human elements." Proc. 14th world Conf. Earthq. Eng., 12-17.

McCormick, J., DesRoches, R., Fugazza, D., and Auricchio, F. (2007). "Seismic assessment of concentrically braced steel frames with shape memory alloy braces." Journal of Structural Engineering, 133(6), 862-70.

McCormick, J., DesRoches, R., Fugazza, D., and Auricchio, F. (2006). "Seismic vibration control using superelastic shape memory alloys.” J Eng Mater Technol, 128,294-301.

Menna, C., Auricchio, F., Asprone, D. (2015). "Applications of shape memory alloys in structural engineering." Elsevier Amsterdam.

Michael, R. J., Johnson, D. H., Pollino, M., Redovan, J., Moser, E., and MacDonald, B. (2012). "Development of a seismic isolation system for commercial storage racks." In: International Mechanical Engineering Conference and Exposition, Houston, TX.

Miller, D. J., Fahnestock, L. A., Eatherton, M. R. (2012). "Development and experimental validation of a nickel-titanium shape memory alloy self-centering buckling-restrained brace." Engineering Structures, 40, 288-98. 
Min, K.W., Seong, J.Y., Kim, J. (2010). "Simple design procedure of a friction damper for reducing seismic responses of a single-story structure." Engineering Structures, 32(11), 3539-3547.

Moschonas, I. F., Kappos, A. J., Panetsoso, P., Papadopoulus, V., Makarios, T., and Thanopoulos, P. (2009). "Seismic fragility curves for Greek bridges: methodology and case studies." $B$. Earthq. Eng., 7 (439-468).

Moss, R. E., Thompson E. M., Kieffer D. S., Tiwari B., Hashash Y. M., Acharya I., Adhikari B. R., Asimaki D., Clahan K. B., Collins B. D., and Dahal, S. (2015). "Geotechnical effects of the 2015 magnitude 7.8 Gorkha, Nepal, earthquake and aftershocks." Seismol. Res. Lett., 86(6), 1514-23.

Nehdi, M., Alam, M.S., and Youssef, M.A. (2010). "Development of corrosion-free concrete beam-column joint with adequate seismic energy dissipation." Engineering Structures, 32(9), 2518-28.

NSC. (2015). http://www.seismonepal.gov.np. National Seismol. Center, Nepal.

Ölander, A. (1932). "An electrochemical investigation of solid cadmium-gold alloys." Am Chem Soc, 54, 3819-33.

OpenSees. 2014 The Open System for Earthquake Engineering Simulation http://opensees.berkeley.edu Pacific Earthq. Eng. Res. Center (PEER)

Ozbulut, O. E., and Hurlebaus, S. (2010). "Evaluation of the performance of a sliding-type base isolation system with a NiTi shape memory alloy device considering temperature effects." Engineering Structures, 32(1), 238-49.

Ozbulut, O. E., and Hurlebaus, S. (2010). "Neuro-fuzzy modeling of temperature-and strain-ratedependent behavior of NiTi shape memory alloys for seismic applications." Journal of Intelligent Material Systems and Structures, 21(8), 837-49.

Ozbulut, O. E., and Hurlebaus, S. (2011). "Energy-balance assessment of shape memory alloybased seismic isolation devices." Smart Struct. Syst., 8(4), 399-412.

Ozbulut, O. E., Hurlebaus, S., and Desroches, R. (2008). "Seismic response control using shape memory alloys: A Review." Journal of Intelligent Materials and Structures, vol. 22, pp. 1531-1549, 2011.

Ozbulut, O. E., Mi, C., Moroni, M. O., Sarrazin, M., and Roschke, P. N. (2007). “A fuzzy model of superelastic shape memory alloys for vibration control in civil engineering applications." Smart Mater. Struct, 16(3) 818.

Ozbulut, O.E., Daghash, S., Sherif, M. M. (2015). "Shape memory alloy cables for structural applications. Journal of Materials in Civil Engineering." 28(4), 04015176.

Parulekar, Y. M., Kiran, A. R., Reddy, G. R., Singh, R. K., and Vaze, K. K. (2014). "Shake table tests and analytical simulations of a steel structure with shape memory alloy dampers." Smart Materials and Structures, 23(12), 125002.

PEER NGA database (2014). "The Pacific Earthquake Engineering Research Center" http://ngawest2.berkeley.edu Pacific Earthq. Eng. Res. Center (PEER). 
Qian, H, Li, H, Song, G., Guo, W. (2013). "Recentering shape memory alloy passive damper for structural vibration control.” Mathematical Problems in Engineering, Nov 28, 2013.

Qian, H., Li, H., and Song, G. (2016). "Experimental investigations of building structure with a superelastic shape memory alloy friction damper subject to seismic loads." Smart Materials and Structures, 25(12), 125026.

Qian, H., Ren, W., Li, H., Song, G., and Chen, H. (2009). "Experimental investigation of steel structure with recentering shape memory alloy damper." In Second International Conference on Smart Materials and Nanotechnology in Engineering, 74936Q-74936Q.

Qiu, C. X., and Zhu, S. (2017). "Performance-based seismic design of self-centering steel frames with SMA-based braces.” Eng. Struct., 130 67-82.

Raghunandan, M., Liel, A. B., and Luco, N. (2015). “Aftershock collapse vulnerability assessment of reinforced concrete frame structures." Earthq. Eng. Struct. Dyn., 44(3) 419-439.

Ramirez, C. M., and Miranda, E. (2012). "Significance of residual drifts in building earthquake loss estimation." Earthq. Eng. Struct. Dyn. 41(11) 1477-93.

Rathje, E. M., Abrahamson, N. A., and Bray, J. D. (1998). "Simplified frequency content estimates of earthquake ground motions." J. Geotech. Geoenviron. Eng. 124(2) 150-159.

Reedlunn, B., Daly, S., Shaw, J. A. (2013). "Superelastic shape memory alloy cables: Part I Isothermal tension experiments." International Journal of Solids and Structures, http://dx.doi.org/10.1016/j.ijsolstr.2013.03.013.

Ren, W., Li, H., and Song, G. (2007). "A one-dimensional strain-rate-dependent constitutive model for superelastic shape memory alloys." Smart Materials and Structures, 16(1), p191.

Ribeiro, F. L., Barbosa, A. R., and Neves, L. C. (2014). "Application of reliability-based robustness assessment of steel moment resisting frame structures under post-mainshock cascading events." J Struct Eng 140(8), A4014008.

Rofooei, F. R., Farhidzadeh, A. (2011). "Investigation on the seismic behavior of steel MRF with shape memory alloy equipped connections." Procedia Engineering, 14, 3325-30.

Ruiz-García J and Negrete-Manriquez J C 2011 Evaluation of drift demands in existing steel frames under as-recorded far-field and near-fault mainshock-aftershock seismic sequences Eng. Struct. 33(2) 621-634

Ruiz-García, J., and Aguilar, J. D. (2015). "Aftershock seismic assessment taking into account postmainshock residual drifts.” Earthq. Eng. Struct. Dyn, 44(9), 1391-407.

Ruiz-García, J., Miranda, E. (2010). "Probabilistic estimation of residual drift demands for seismic assessment of multi-story framed buildings." Eng. Struct. 32 (11-20).

Sabelli, R., Mahin, S., Chang, C. (2003). "Seismic demands on steel braced frame buildings with buckling-restrained braces." Eng. Struct. 25 (655-666).

Saiidi, M.S., and Wang, H. (2006). "Exploratory study of seismic response of concrete columns with shape memory alloys reinforcement." ACI structural journal, 103(3), 436. 
San Juan, J., and Nó, M. L. (2003). "Damping behavior during martensitic transformation in shape memory alloys." Journal of Alloys and Compounds, 355(1), 65-71.

Sause, R., Ricles, J., Mahvashmohammadi, A., Michael, R., Sweeney, S., and Ferro, E. (2012). "Advanced compressed elastomer dampers for earthquake hazard reduction to steel frames." Behaviour of Steel Structures in Seismic Areas (STESSA), Santiago, Chile.

Sepúlveda, J., Boroschek, R., Herrera, R., Moroni, O., and Sarrazin, M. (2008). "Steel beamcolumn connection using copper-based shape memory alloy dampers." Journal of Constructional Steel Research, 64(4), 429-35.

Shcherbakov, R., Nguyen, M., and Quigley, M. (2012). "Statistical analysis of the 2010 MW 7.1 Darfield Earthquake aftershock sequence." New Zeal. J. Geol. Geophys., 55(3), 305-311.

Sheather S. J. (2008). "A modern approach to regression with R. Springer, New York.

Shinozuka, M., Chaudhuri, S. R., Mishra, S. K. (2015). "Shape-Memory-Alloy supplemented Lead Rubber Bearing (SMA-LRB) for seismic isolation.” Probabilistic Engineering Mechanics, 41, 34-45.

Shinozuka, M., Feng, M. Q., Kim, H. K., and Kim, S. H. (2000). "Nonlinear static procedure for fragility curve development." J. Eng. Mech., 126 (1287-1296).

Shinozuka, M., Feng, M. Q., Lee, J., and Naganuma, T. (2000). "Statistical analysis of fragility curves." J. Eng. Mech. 126 (1224-1231).

Silwal, B., Michael, R. J., and Ozbulut, O. E. (2015). “A superelastic viscous damper for enhanced seismic performance of steel moment frames." Eng. Struct, 105, 152-64.

Silwal, B., Ozbulut, O. E., and Michael, R. J. (2016). "Seismic collapse evaluation of steel moment resisting frames with superelastic viscous damper.” J. Constr. Steel Res., 126, 26-36.

Song, G., Ma, N., Li, H. N. (2006). "Applications of shape memory alloys in civil structures." Engineering structures, 28(9), 1266-74.

Song, J., and Ellingwood, B. R. (1999). "Seismic reliability of special moment steel frames with welded connections: I and II." J. Struct. Eng. 125 (357-384).

Song, R., Li, Y., and van de Lindt, J. W. (2014). "Impact of earthquake ground motion characteristics on collapse risk of post-mainshock buildings considering aftershocks." Eng. Struct. 81, 349-361.

Speicher, M. S., DesRoches, R., Leon, R. T. (2011). "Experimental results of a NiTi shape memory alloy (SMA)-based recentering beam-column connection." Engineering structures, 33(9), 2448-57.

Speicher, M., Hodgsonm D. E., DesRoches, R., Leonm R. T. (2009). "Shape memory alloy tension/compression device for seismic retrofit of buildings." Journal of materials engineering and performance, 18(5-6), 746-53.

Sweeney, S. K., and Michael, R. J. (2006). "Collaborative product realization of an innovative structural damper and application.” In: Proc. ASME International Mechanical Engineering Congress, IMECE2006-13421, 1-9, Chicago, IL, November 2006. 
Tyber, J., McCormick, J., Gall, K., DesRoches, R., Maier, H.J. and Abdel Maksoud, A.E. (2007). "Structural engineering with NiTi. I: basic materials characterization." Journal of Engineering Mechanics, 133(9), 1009-1018.

Uma, S. R., Pampanin, S., and Christopoulos, C. (2010). "Development of probabilistic framework for performance-based seismic assessment of structures considering residual deformations." J. Earthq. Eng. 14(7), 1092-111.

Uriz, P., and Mahin, S. (2008). "Toward earthquake-resistant design of concentrically braced steelframe structures, PEER Report 2008/08." Pacific Earthquake Engineering Research Center, University of California, Berkeley, CA, USA.

USGS. (2000). "Implications for earthquake risk reduction in the United States from the Kocaeli, Turkey, earthquake of August 17, 1999.” U.S. Geol. Surv., Circular 1193.

USGS. (2017). "Advanced National Seismic System Current Status, Development Opportunities, and Priorities for 2017-2027." USGS Circular 1429.

Vamvatsikos, D., and Cornell, C. A. (2002). "Incremental dynamic analysis." Earthq. Eng. Struct. Dyn, 31(3), 491-514.

Van Humbeeck, J. (2003). "Damping capacity of thermoelastic martensite in shape memory alloys." Journal of Alloys and Compounds, 355(1), 58-64.

Wang, W., Fang, C. and Liu, J. (2016). "Large size superelastic SMA bars: heat treatment strategy, mechanical property and seismic application." Smart Materials and Structures, 25(7), p.075001.

Wang, Z. G., Zu, X. T., Dai, J. Y., Fu, P., and Feng, X. D. (2003). "Effect of thermomechanical training temperature on the two-way shape memory effect of TiNi and TiNiCu shape memory alloys springs." Materials Letters, 57(9), pp.1501-1507.

Wanitkorkul, A., and Filiatrault, A. (2008). "Influence of passive supplemental damping systems on structural and nonstructural seismic fragilities of a steel building" Eng. Struct, 30 (675$682)$.

Wei, C. Y. (2006). "A study of local-buckling BRB and cost performance of BRBF, MS Thesis." National Taiwan University, Taipei.

Wikiwand. "List of earthquakes in 1985, damage in Mexico City after the 1985 earthquake." http://www.wikiwand.com/en/List_of_earthquakes_in_1985

Wilson, J. C., and Wesolowsky, M. J. (2005). "Shape Memory Alloys for Seismic Response Modification: A State-of-the-Art Review." Earthquake Spectra, 21(2), 569-601.

Wolons, D., Gandhi, F., and Malovrh, B. (1998). "Experimental investigation of the pseudoelastic hysteresis damping characteristics of shape memory alloy wires." Journal of Intelligent Material Systems and Structures, 9(2), pp.116-126.

Wu, K., Yang, F., Pu, Z., and Shi, J. (1996). "The effect of strain rate on detwinning and superelastic behavior of $\mathrm{Ni} \mathrm{Ti}$ shape memory alloys." Journal of intelligent material systems and structures, 7(2), pp.138-144. 
Yam, M. C., Fang, C., Lam, A. C., Zhang, Y. (2015). "Numerical study and practical design of beam-to-column connections with shape memory alloys." Journal of Constructional Steel Research, 104,177-92.

Yamazaki, F., Hamada, T., Motoyama, H., Yamauchi, H. (1999). "Earthquake damage assessment of expressway bridges in Japan." Tech. Council Lifeline Earthq. Eng. Monograph 1999, 361-370.

Yang, C. S., DesRoches, R., Leon, R. T. (2010). "Design and analysis of braced frames with shape memory alloy and energy-absorbing hybrid devices.” Engineering Structures, 32(2), 498507.

Yin, H., and Sun, Q. (2012). "Temperature variation in NiTi shape memory alloy during cyclic phase transition." Journal of materials engineering and performance, 21(12), pp.25052508.

Youssef, M. A., Alam, M. S., Nehdi, M. (2008). "Experimental investigation on the seismic behavior of beam-column joints reinforced with superelastic shape memory alloys." Journal of Earthquake Engineering, 12(7), 1205-22.

Zhang, Y., and Zhu, S. (2007). "A shape memory alloy-based reusable hysteretic damper for seismic hazard mitigation." Smart Mater. Struct. 16(5), 1603.

Zhu, S., and Zhang, Y. (2008). "Seismic analysis of concentrically braced frame systems with selfcentering friction damping braces." Journal of Structural Engineering, 134(1), 121-31. 


\section{VITA}

Baikuntha Silwal received his Bachelor of Engineering in Civil Engineering from Tribhuvan University, Nepal, in 2000. He worked as a civil/structural engineer for 10 years, for civil engineering projects in enhancing the performance of bridge, building and steel tower structures subjected to wind and seismic hazards. At the Southern Illinois University at Carbondale, he enrolled in the Civil Engineering program, with an emphasis on Structural Engineering, beginning in January 2011. He obtained his Master of Science degree in May 2013. He then enrolled as a Ph.D. student in the Structural Engineering program at The University of Virginia in January 2013.

Baikuntha Silwal may be contacted at the Department of Civil Engineering, The University of Virginia, Charlottesville, VA 22904. His personal email address is baikunthanp@ hotmail.com. 